Christoph Bräuer (Hrsg.)

\title{
Denkrahmen der Deutschdidaktik
}

Die Identität der Disziplin in der Diskussion

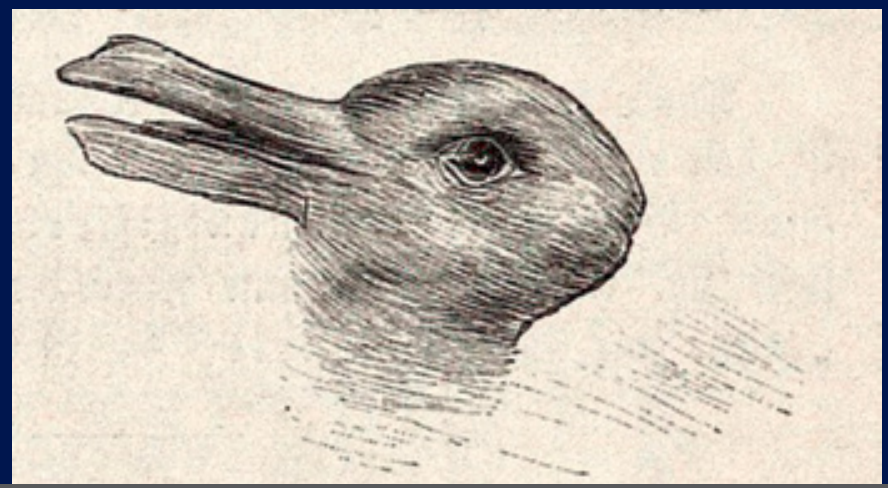




\section{Christoph Bräuer (Hrsg.)}

\section{Denkrahmen der Deutschdidaktik}

Auch wenn die Deutschdidaktik sich endgültig als akademische Disziplin etabliert hat, gehen die Meinungen, welchen Stellenwert sie in der Ausbildung von Professionalität oder Lehrkompetenz haben könnte, auseinander. Weder gibt es Konsens darüber, was die Hochschulbildung zur Professionalisierung leisten kann, noch ist der systematische Ort im Rahmen der wissenschaftlichen Disziplinen geklärt. Der Band greift diesen Selbstverständigungsprozess auf und lenkt den Blick sowohl auf die geschichtliche Entwicklung, die gegenwärtige Gestaltung als auch auf die zukünftige Ausrichtung. Mit der Kompetenzorientierung sind neben den an Inhalten zu erwerbenden Kompetenzen von Schülerinnen und Schülern auch die von zukünftigen Lehrkräften zu erwerbenden Kompetenzen in den Blick geraten. Zugleich wird die kompetente Lehrperson als Faktor effektiven (Fach-) Unterrichts stärker wahrgenommen und mit dieser Fokussierung der Wunsch nach einer fachdidaktischen Profilierung der Lehramtsausbildung verbunden.

\section{Der Herausgeber}

Christoph Bräuer ist Professor für Didaktik der Deutschen Sprache und Literatur an der Universität Göttingen und Leiter der Sammlung historischer Kinder- und Jugendliteratur. Er studierte die Fächer Deutsch und Geschichte auf Lehramt für Gymnasien und wurde mit dem „Förderpreis Deutschdidaktik" ausgezeichnet. Seine Forschungsschwerpunkte sind fachdidaktische Unterrichtsforschung, Leseund Schreibausbildung sowie literarische Rezeptionsprozesse. 


\section{Denkrahmen der Deutschdidaktik}




\section{POSITIONEN DER DEUTSCHDIDAKTIK THEORIE UND EMPIRIE \\ Herausgegeben von Christoph Bräuer und Iris Winkler}

\section{BAND 1}

\section{PETER LANG}


Christoph Bräuer (Hrsg.)

\section{Denkrahmen \\ der Deutschdidaktik}

Die Identität der Disziplin in der Diskussion

\section{PETER LANG}




\title{
Bibliografische Information der Deutschen Nationalbibliothek
}

Die Deutsche Nationalbibliothek verzeichnet diese Publikation in der Deutschen Nationalbibliografie; detaillierte bibliografische Daten sind im Internet über http://dnb.d-nb.de abrufbar.

\author{
Umschlagabbildung: \\ (c) http://digi.ub.uni-heidelberg.de/diglit/fb97/0147 / \\ Universitätsbibliothek Heidelberg / Fliegende Blätter 97 (1892) S. 147
}

ISSN 2364-1312

ISBN 978-3-631-66138-3 (Print)

E-ISBN 978-3-653-05696-9 (E-PDF)

E-ISBN 978-3-631-70204-8 (EPUB)

E-ISBN 978-3-631-70205-5 (MOBI)

DOI 10.3726/ b10881

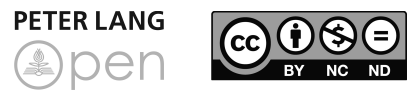

Open Access: Dieses Werk ist lizensiert unter der Creative Commons Lizenz Namensnennung - Nicht kommerziell - Keine Bearbeitungen 4.0 International (CC BY-NC-ND 4.0). Den vollständigen Lizenztext finden Sie unter: https://creativecommons.org/licenses/by-nc-nd/4.0/deed.de

(C) Christoph Bräuer, 2016

Peter Lang GmbH

Internationaler Verlag der Wissenschaften

Peter Lang - Berlin · Bern · Bruxelles .

New York · Oxford $\cdot$ Warszawa $\cdot$ Wien

Diese Publikation wurde begutachtet.

www.peterlang.com 


\section{Inhaltsverzeichnis}

Vorwort

Christoph Bräuer

Einleitung - (k)ein Denkrahmen für die Deutschdidaktik? 9

Christoph Bräuer

Deutschdidaktik - (k)ein Denkkollektiv ohne Denkstil?

Juliane Köster

Die dilemmatische Disziplin - Deutschdidaktik zwischen Eklektizismus

und Partialisierung

Torsten Pflugmacher

Abstand durch Nähe - Nähe durch Abstand. Deutschdidaktik als

reflexive Wissenschaft

Marcus Steinbrenner

„Die Sprachlichkeit des Menschen als Bildungsaufgabe in der Zeit" und als Denkrahmen für die Deutschdidaktik

Dorothee Wieser

Zum Verhältnis von Rekonstruktion, Konstruktion und Normfragen in der Deutschdidaktik

Jakob Ossner

Didaktische Konstrukte und Konstruktvalidität in der Deutschdidaktik

Iris Winkler

Deutschdidaktik - eine Anwendungswissenschaft? 169

Gerhard Rupp

Deutschdidaktik - eine eingreifende Kultur- und kompetenzorientierte Vermittlungswissenschaft 
Harro Müller-Michaels

Denken in Ellipsen - Didaktik im Spannungsfeld von Bildung und Wissenschaften

Michael Krelle

Deutschdidaktik als (zu) gestaltende Wissenschaft?

229 


\section{Vorwort}

Der vorliegende Band versammelt die Beiträge einer Arbeitstagung zum Thema „Denkrahmen der Deutschdidaktik - Positionen in der Diskussion“, die am 24. und 25. März 2015 an der Georg-August-Universität Göttingen stattfand. Diese Tagung machte es sich zur Aufgabe, die vielfältigen Denkanstöße Jakob Ossners zur Frage einer disziplinären Identität der Didaktik Deutsch aufzugreifen und sie wäre daher ohne ihn, seine Unterstützung und seine Beteiligung nicht denkbar gewesen. Der Band ist daher Jakob Ossner gewidmet.

Ich bin in die Fragen nach der Identität des Faches und in die Herausforderungen einer disziplinären Selbstvergewisserung während meines Studiums an der Goethe-Universität Frankfurt eingeführt worden. In Frankfurt hat die Frage nach dem Denkrahmen eine lange Tradition, die in besonderer Weise mit dem Namen Hubert Ivo verknüpft ist. Jakob Ossner hat die Frage des disziplinären Selbstverständnisses weitergeführt, virulent ist sie für mich bis heute geblieben.

Aber die Auseinandersetzung mit der eigenen Disziplin ist keine Frankfurter Tradition, sie ist auch keine Veranstaltung der Vergangenheit und sie erhält von älteren ebenso wie von jüngeren Didaktikerinnen und Didaktikern wegweisende neue Impulse. Ihre fachlichen und institutionellen Voraussetzungen liegen in ihrer Geschichte begründet, ihre Argumentation ist in der bildungs- und hochschulpolitischen Gegenwart verankert, ihre Perspektive aber auf eine Zukunft gerichtet, in der fachliches Wissen und Können mehr denn je bedeutsam sein werden.

In der Vorbereitung der Tagung zeigte sich die Problematik der Frage nach der disziplinären Identität in aller Deutlichkeit - in der Verortung zwischen den Bezugsdisziplinen der Fachwissenschaften und Bildungswissenschaften, im Spannungsfeld zwischen theoretischen Erkenntnissen und praktischer Anwendung, in der Komplexität und Diversität der möglichen und nötigen Zugänge zur vorliegenden Fragestellung.

Für die Beitragenden der Tagung stellte die Auseinandersetzung mit dieser Frage eine positive Herausforderung dar - sie haben die Tagung ermöglicht und getragen durch ihre Beiträge und Diskussionen. Ihre Positionen sind in diesem Band im Duktus einer lebendigen Debatte versammelt. Auch und gerade ihnen gilt mein besonderer Dank.

Schließlich gestaltete sich die Arbeitstagung auch deshalb so anregend, weil jeweils mit einer kritisch-konstruktiven Respondenz zu je zwei Beiträgen in die Diskussion eingeleitet wurde - der Dank gilt hier namentlich Ricarda Freudenberg, 
Silke Kubik, Diana Maak und Frederike Schmidt. In der Vorbereitung und Durchführung haben mich Frerk Schäfers und Claudia Gärtner sowie Ellen Schneidereit unterstützt und so maßgeblich zu einer reibungslosen und gelungenen Tagung beigetragen.

Nicht zuletzt ist der Friedrich Stiftung sowie dem Unibund Göttingen e.V. sehr herzlich für ihre großzügige finanzielle Unterstützung der Arbeitstagung zu danken, ohne die ein solches produktives und wichtiges Unterfangen nicht möglich gewesen wäre.

Mit diesem Sammelband liegen die Debattenbeiträge der Arbeitstagung nun auch in Schriftform vor - dies gelang nur durch die tatkräftige Unterstützung von Christina Schmidt und Robert Halagan, mein letzter Dank gebührt daher ihnen.

Göttingen, im Oktober 2016

Christoph Bräuer 


\title{
Christoph Bräuer \\ Einleitung - (k)ein Denkrahmen für die Deutschdidaktik?
}

\author{
Was heißt und zu welchem Ende studiert man Universalgeschichte?
}

So abgegriffen dieser vielfach zitierte Titel aus Friedrich Schillers akademischer Antrittsrede in Jena 1789 klingen mag, er ist es eben auch deshalb, weil er damals wie heute zentrale Fragen einer akademischen Disziplin, zumal einer damals noch ganz jungen wie der Geschichtswissenschaft respektive einer heute immer noch recht jungen wie der Didaktik Deutsch, trifft: Die Frage nach der Identität, nach dem, was sie auszeichnet, und das heißt auch, was sie untersucht, beforscht und lehrt, auf der einen Seite, und die Frage nach dem „cui bono“, nach dem Ziel und der Funktion einer solchen Disziplin auf der anderen Seite.

Schiller wendet sich im weiteren Verlauf seiner Rede gegen ein allzu praktisch veranlagtes Studien- und Ausbildungsinteresse, eine „Brodgelehrtheit“. Er dagegen fordert „philosophische Köpfe“, denn der „Brodgelehrte“ werde „seinen ganzen Fleiß [...] nach den Foderungen einrichten, die von dem künftigen Herrn seines Schicksals an ihn gemacht werden, und alles gethan zu haben glauben, wenn er sich fähig gemacht hat, diese Instanz nicht zu fürchten“. Wenn dieses Ziel erreicht ist, wird „seine größte Angelegenheit [...] [sein], die zusammen gehäuften Gedächtnißschätze zur Schau zu tragen, und ja zu verhüten, dass sie in ihrem Werthe nicht sinken. Jede Erweiterung seiner Brodwissenschaft beunruhigt ihn, weil sie ihm neue Arbeit zusendet, oder die vergangene unnütz macht; jede wichtige Neuerung schreckt ihn auf, denn sie zerbricht die alte Schulform, die er sich so mühsam zu eigen machte, sie setzt ihn in Gefahr, die ganze Arbeit seines vorigen Lebens zu verlieren.“

Schiller spricht hier weder über Lehrerbildung im Allgemeinen noch über angehende (Deutsch)Lehrende im Besonderen, doch mag es sich seltsam bekannt anhören für die Ohren von DeutschdidaktikerInnen. Schillers Kritik an einer zu kurz gedachten Anwendungs- oder Praxisorientierung in der universitären Lehre ist jedenfalls ebenso deutlich wie anschlussfähig an die heute oft an die Didaktik herangetragenen Forderungen wie seine Kritik an einem Verharren auf einmal aufgestellten Lehrsätzen zeitlos. 
Als eine wissenschaftliche Disziplin bestimmt sich die Deutschdidaktik über ihr spezifisches Forschungsfeld und nicht (ausschließlich) über ihr praktisches Anwendungsgebiet.

Schiller fordert „philosophische Köpfe“, Individuen, die ihr Wissen und NichtWissen und dessen Möglichkeiten und Grenzen kennen und zum Gegenstand gezielten Nachdenkens und forschenden Lernens machen können. Und die dennoch oder deshalb - handlungsfähig werden und bleiben.

Wie muss eine Disziplin verfasst sein, um „philosophische Köpfe“ - als reflektierte PraktikerInnen - auszubilden? Wie ,theoretisch' muss und wie ,praktisch' kann eine wissenschaftliche Disziplin sein, um als Wissenschaft zu gelten und für die Praxis bilden? Es geht bei der Frage nach der Identität einer pädagogischen oder didaktischen Disziplin als Wissenschaft letztlich auch um „den Nachweis der Bedeutung der pädagogischen Theorie für die Praxis“ (Weniger 1952) - jedenfalls dann, wenn man die Disziplin nicht ganz aus der Verantwortung für das praktische Anwendungsgebiet herauslösen möchte.

Auf diese „Probleme einer akademischen Lehrerbildung" versucht Erich Weniger, wiederum in seiner Antrittsvorlesung, in Hinblick auf sein Fach, die Pädagogik, eine Antwort:

Aber die wissenschaftliche Theorie der Pädagogik hat nicht bloß diese objektive, bloß analytische Funktion der Aufklärung des Sachverhalts, sondern sie hat auch einen Ort unmittelbar im Zusammenhang der Praxis selbst. Sie übernimmt die Funktion der Theorie innerhalb der Praxis als stellvertretende Besinnung, als Läuterung der in der Praxis angelegten Theorien, als bewusste Vorbesinnung und bewusste nachträgliche Klärung. Während sie als Theorie der Theorie, beschäftigt mit der Aufklärung des Gesamtsachverhaltes von Theorie und Praxis, sozusagen für sich bestehen kann, von außen und von oben das Geschehen auf dem Felde der Erziehung betrachtend, ist sie hier innerlich an die Praxis gebunden, ist sie schlechthin abhängig von der Praxis und der Erziehungswirklichkeit. Sie dient der Praxis und gilt nur so weit, als sie der Praxis helfen kann. Es gilt hier der Primat der Praxis, die mit Theorie geladen, doch an sich unabhängig ist von der Pädagogik als Wissenschaft. (Weniger 1952, S. 20).

Für Weniger liegt die Voraussetzung für die „Funktion der Theorie in der Praxis“ in der „Befangenheit des Theoretikers in der pädagogischen Aufgabe und an das Pädagogische Tun“ (ebd., S. 21); die WissenschaftlerInnen müssten „die Verantwortung der Praxis teilen, ihre Ziele bejahen, von der Verantwortung und von den Zielen aus denken“, damit sie ihre Aufgaben in den Blick bekämen: „Man sieht nur als Befangener“ (ebd.). Für Weniger schließt diese Befangenheit ein, dass „auch der pädagogische Theoretiker die pädagogische Haltung besitzen und das pädagogische Ethos in seinem theoretischen Denken verwirklichen muß“(ebd.). Dann kann die theoretische Bildung der Universität den 
Nachwuchs lehren, Erfahrungen zu machen und [...] ihm zu der Einstellung verhelfen, aus der Erfahrungen und die „Kritik der langen Mühen“ erwachsen. Heute kommt es bei der Neugestaltung der Volksschule auf die Einsicht und den bewussten Willen jedes einzelnen Erziehers an, die Ausbildung soll ihm die Kraft und die Freiheit dazu geben und die Hilfsmittel, die es ihm erleichtern können, aber keine Vorschriften und keine überheblichen Prinzipien (Weniger 1952, S. 22).

Ist dies der Weg über theoretische Bildung zu einem „philosophischen Kopf“ zu werden?

Antrittsvorlesungen sind programmatisch: In Schillers Rede spürt man das Idealistische, in Wenigers das Pathetische.

Ob sich eine Wissenschaft tatsächlich dem „Primat der Praxis“ unterstellen möchte, wie sie ihr Verhältnis von „Theorie“ (oder auch Grundlagen-Forschung) und „Praxis“ (oder auch Anwendung) bestimmt, welche Ziele sie sich setzt, betrifft die Frage ihres disziplinären Selbstverständnisses, ihrer disziplinären Identität.

In diesem Band sind Beiträge versammelt, die oft ähnliche, aber auch gegensätzliche Positionen in Hinblick auf die Disziplin Deutschdidaktik beziehen programmatisch ist der Band nur insofern, als er die Fragen nach dem „Was heißt und wozu studiert man Deutschdidaktik?" für ebenso aktuell wie zeitlos hält.

Der vorliegende Band beschäftigt sich mit der Frage nach dem oder den möglichen Denkrahmen einer genuin deutschdidaktischen Forschung und Lehre. Dieser Selbstverständigungsprozess einer wissenschaftlich noch immer relativ jungen Disziplin begleitet die Fachdidaktik Deutsch seit ihrer Etablierung. Abgrenzungs- und Selbstbestimmungsversuche fanden und finden im Spannungsfeld von historischen, politischen und institutionellen Rahmenbedingungen statt (vgl. Ivo 1977).

Eine Beschäftigung mit Blick auf Vergangenheit, Gegenwart und Zukunft erscheint zum gegenwärtigen Zeitpunkt besonders notwendig und gewinnbringend: In den vergangenen zehn Jahren hat die Deutschdidaktik in der Folge der großen Vergleichsstudien und der Diskussion um Kompetenzen und Standards personell und institutionell eine deutliche Aufwertung erfahren. Deutschdidaktische Inhalte haben verstärkt Berücksichtigung in den neu eingeführten BA-MA-Studiengängen im Rahmen aller Schulstufen gefunden. Mit der Kompetenzorientierung sind neben den an Inhalten zu erwerbenden Kompetenzen von Schülerinnen und Schülern zunehmend auch die von den zukünftigen Lehrkräften sich anzueignenden Kompetenzen in den Blick von Forschung und Lehre geraten (vgl. Bräuer/Wieser 2015). Zugleich wird die kompetente Lehrperson als Faktor eines effektiven (Fach-)Unterrichts wieder stärker herausgestellt (vgl. Hattie 2013), und damit verbunden die Anforderung einer fachdidaktischen Profilierung und Professionalisierung der Lehramtsausbildung. 
Mit diesen Veränderungen gehen nicht nur eine verstärkte empirische Ausrichtung der fachdidaktischen Forschung einschließlich der Adaption und Integration neuer Forschungsansätze, Fragestellungen und -methoden einher, sondern auch die Etablierung einer neuen fachdidaktisch forschenden Generation von NachwuchswissenschaftlerInnen. Diese Auf- und Umbruchsituation verlangt nach einem intensiven Diskurs über das disziplinäre Selbstverständnis der Deutschdidaktik, der alle deutschsprachigen Länder einbezieht.

Vor diesem Hintergrund spiegeln die Beiträge in diesem Band nicht nur die verschiedenen Generationen fachdidaktischer Forschung und Lehre, sondern auch die beiden großen disziplinären Teilbereiche Literatur und Sprache. Inwieweit finden diese unterschiedlichen Akteurinnen und Akteure mit ihren vielfältigen Perspektiven unter dem Dach oder im Namen einer Disziplin zueinander und zusammen? Diese disziplinäre Kernfrage steht im Zentrum des Bandes, im Titel umschrieben als die Frage nach dem „Denkrahmen“ - um sie zu bearbeiten, kann die Frage nach dem, was disziplinäre Gemeinschaften auszeichnet, hilfreich sein: Ludwik Fleck hat für eine solche Gemeinschaft den Begriff des „Denkkollektivs“ vorgeschlagen, verstanden als "Gemeinschaft der Menschen, die im Gedankenaustausch oder in gedanklicher Wechselwirkung stehen " (Fleck 1999, S. 54 f.). In einem solchen Denkkollektiv sieht Fleck „den Träger geschichtlicher Entwicklung eines Denkgebietes, eines bestimmten Wissensbestandes und Kulturstandes, also eines besonderen Denkstils" (ebd.).

Mit der Suche nach dem oder den Denkrahmen der Deutschdidaktik wird nach den vorfindbaren oder fehlenden „Denkkollektiven“ und ihren „Denkstilen“ gefragt - bisher, so scheint es, fehlt der Deutschdidaktik ein als kollektiv geteilt geltender Denkstil. Und somit, trotz ihrer endgültigen institutionellen Etablierung als akademischer Disziplin, ein verbindlicher Denkrahmen.

Harro Müller-Michaels beschreibt die Deutschdidaktik schon 1972 als eingreifende "normsetzende Handlungswissenschaft“. Auch Hubert Ivo hat sich immer wieder auf die Suche nach dem Kern der disziplinären Identität gemacht (Handlungsfeld Deutschunterricht: Argumente und Fragen einer praxisorientierten Wissenschaft, 1975; Zur Wissenschaftlichkeit der Didaktik der deutschen Sprache und Literatur, 1977), in Festschriften zu seinen Ehren sind ihm Kolleginnen und Kollegen auf dieser Suche gefolgt (Bremerich-Vos 1993; Gölitzer/Roth 2007). Ivo hat vorgeschlagen, an Stelle einer Fachdidaktik als Vermittlungs-, Reflexions-, Integrations- oder Unterrichtswissenschaft die Didaktik der deutschen Sprache und Literatur als „Fachunterrichtswissenschaft“ zu bestimmen (1977). Die Positionen der Deutschdidaktik seit 1949 hat Müller-Michaels 1980 zusammengestellt - der Reihentitel aktualisiert diese 
Perspektive auf die eigene Disziplin und schreibt sie fort. Seitdem sind weitere Positionierungen vorgeschlagen worden: So hat etwa Jakob Ossner $(1993 ; 2001)$ die Deutschdidaktik als „praktische Wissenschaft“ und als „Handlungswissenschaft" skizziert. Diese Suchbewegungen sind jedoch nach allen Seiten offen geblieben, und entsprechend versteht sich die Deutschdidaktik mitunter selbst (noch) als „Wissenschaft zwischen den Stühlen“ (Rosebrock/Fix 2001). Matthis Kepser $(2013$, S. 52) etwa setzt sich kritisch mit diesen Bestimmungsversuchen auseinander, um dann mit „Deutschdidaktik als eingreifende Kulturwissenschaft“ seinerseits einen neuen, eigenen „Positionierungsversuch im wissenschaftlichen Feld zu versuchen“.

Ein weiterer Ausdruck dieser unbestimmten disziplinären Identität zeigt sich darin, dass in den vergangenen anderthalb Jahrzehnten zwar zahlreiche „Einführungen " in die jeweils von den Autoren favorisierte Spielart der Sprach-, Literatur- oder Mediendidaktik erschienen sind, es aber an übergreifend gültigen Handbüchern fehlt. Ludwik Fleck hat das „Handbuch“ als dasjenige Medium bezeichnet, welches den von allen geteilten, geltenden Stand einer Disziplin widerspiegele. Handbücher, so schreiben die Autoren des Handbuchs Didaktik der deutschen Sprache, „wachsen aus der Tiefe der Disziplin“ - ihr Handbuch, so stellen sie fest, „kann in dieser verhältnismäßig jungen Forschungsdisziplin [...] nicht einfach den klassischen Stand zusammenfassen, sondern muss ihn zunächst einmal als solchen etablieren, in der Hoffnung, dass er von der Scientific community als wissenschaftlicher Gegenstand anerkannt werde" (Bredel et al. 2003, S. 11). Inwieweit dieses zweibändige Werk seit seinem Erscheinen vor 13 Jahren den Status eines „Handbuchs“ erwerben konnte, bliebe zu diskutieren.

Am Beispiel von Einführungen und Handbüchern zeigt sich noch in anderem Zusammenhang die Relevanz einer disziplinären Selbstvergewisserung: Je nach ihrer (impliziten) Positionierung führen die vorliegenden Einführungen mehr oder weniger ausschließlich in die Kompetenzbereiche des Unterrichtsfachs ein. Andere Bereiche deutschdidaktischer Forschung, etwa zu Motiven der Berufswahl und zu Lehrerbiografien, zu epistemologischen Überzeugungen oder zu Unterrichtsrekonstruktion und -reflexion, finden ebenso wie Forschungsmethoden und Auswertungsverfahren nur am Rande Aufmerksamkeit - obwohl diese Felder in den vergangenen Jahren verstärkt auch in der Deutschdidaktik beforscht bzw. bearbeitet werden. Der Fokus der Einführungen liegt so überwiegend auf dem Bereitstellen praxisbezogenen - und das meint unterrichtsbezogenen - Wissens und weniger auf der Einführung in die akademische Disziplin.

Bildet die Disziplin doch eher „Brodgelehrte“ denn „philosophische Köpfe“ aus? Wie ist es um den Nachweis didaktischer Theorie(bildung) für die nachhaltige 
Bildung künftiger Lehrender wie Forschender bestellt? Die Verhältnisbestimmung von praxisorientierter und forschungsbezogener Lehre bleibt virulent - gerade in Zeiten zunehmenden kompetitiven Wettbewerbs um Forschungsgelder und Lehrressourcen wie um Studierende und Ausbildungsgänge.

Ziel eines solchen Sammelbandes kann es nicht sein, das wissenschaftliche Profil einer Deutschdidaktik herauszuarbeiten. In der Zusammenschau der Beiträge zeigt sich aber, wie einzelne Positionen profiliert und kontrastiert, Gemeinsamkeiten herausgestellt und disziplinäre Ideen und Utopien entwickelt werden. Auf der Grundlage dieses Problemaufrisses seien die einzelnen Beitragenden und ihre Beiträge kurz vorgestellt:

Christoph Bräuer wendet in seinem Beitrag „Deutschdidaktik - (k)ein Denkkollektiv ohne Denkstil?" den wissenssoziologischen Denkstil-Begriff von Ludwik Fleck einerseits rekonstruktiv auf die wissenschaftliche Wahrnehmung des didaktischen Handlungsfeldes an und arbeitet so Voraussetzungen und Fokussierungen didaktischer Zugriffe auf die Praxis heraus. Er wendet die ,Lehre vom Denkstil' andererseits konstruktiv so, dass darin die fachlichen Lehr-Lern-Prozesse als Koordination von Zeigen und Sehen-Lassen zum genuinen Gegenstand einer Deutschdidaktik als praktischer Wissenschaft werden können.

Juliane Köster zeigt in ihrem Beitrag „Die dilemmatische Disziplin - Deutschdidaktik zwischen Eklektizismus und Partialisierung " zwei Reaktionsweisen der Disziplin auf die fehlende Einheit des Faches: Zum einen arbeitet Köster die zunehmende Aufspaltung in zahlreiche Teilgruppierungen mit sehr unterschiedlichen Denkstilen heraus, zum anderen zeichnet sie den Versuch nach, mit Hilfe transdisziplinärer Dachkonzepte eine Einheit zu konstruieren. Abschließend plädiert Köster für den Mut, die Didaktik Deutsch als Professionswissenschaft in Analogie zur Rechtswissenschaft und Medizin zu bestimmen.

Torsten Pflugmacher schlägt in seinem Beitrag „Abstand durch Nähe - Nähe durch Abstand. Deutschdidaktik als reflexive Wissenschaft" eine Bestimmung als Reflexionswissenschaft vor. Pflugmacher fordert eine fachdidaktische Grundlagenforschung, die sich als Gegengewicht einer evidenzbasierten Outcome- bzw. Outputorientierung auf die beschreibende Rekonstruktion von literarischen Vermittlungs- und Aneignungsprozessen konzentriert. Auf der Grundlage dieser Rekonstruktionsforschung verlangt er eine Theoriebildung, die sich nicht an den normativen Zielsetzungen eines (kompetenzorientierten) Literaturunterrichts ausrichtet, sondern ihren Ausgang in der Rekonstruktion der immanenten Normativität durch die gesprächssequenzielle Analyse von Unterrichtskommunikation sieht. 
Marcus Steinbrenner geht in seinem Beitrag „,Die Sprachlichkeit des Menschen als Bildungsaufgabe in der Zeit' - und als Denkrahmen für die Deutschdidaktik" von Wilhelm von Humboldts und Eugenio Coserius Sprachdenken aus und schließt an die zentralen Arbeiten von Hubert Ivo zur Denkrahmenthematik an. Mit diesem anthropologisch, bildungs- und sprachtheoretisch fundierten Denkrahmen nimmt Steinbrenner aktuelle deutschdidaktische Theorieansätze kritisch in den Blick und skizziert ein Konzept sprachlich-literarischer Bildung in neun Aspekten, in dessen Zentrum die „Freiheit des poetischen Sprechens“ (Jürgen Trabant) steht.

Dorothee Wieser untersucht in ihrem Beitrag „Zum Verhältnis von Rekonstruktion, Konstruktion und Normfragen in der Deutschdidaktik" das Zusammenspiel von Konstruktion, Rekonstruktion und Normfragen, die sie als Eckpunkte des deutschdidaktischen Handlungsfeldes versteht. Sie entwickelt in ihrem Beitrag die Notwendigkeit, diese drei Aspekte stets mitzudenken und mitzureflektieren, auch wenn jeweils unterschiedliche Schwerpunkte gesetzt werden können.

Jakob Ossner untersucht „Didaktische Konstrukte und Konstruktvalidität in der Deutschdidaktik“. In seinem Beitrag zeigt er an didaktischen Modellierungen im Bereich des Schriftspracherwerbs die Implikationen der jeweiligen Konstruktvalidität und arbeitet heraus, dass es Aufgabe einer Deutschdidaktik ist, Lehrkräften klare Konstrukte an die Hand zu geben, auf deren Grundlage sie didaktische Entscheidungen des Unterrichtens und eine angemessene und zielführende Material- und Methodenwahl treffen können.

Iris Winkler geht in ihrem Beitrag „Deutschdidaktik - eine Anwendungswissenschaft?" der Frage nach, wie die Diskrepanz zwischen dem fachlichdidaktischen Wissen der akademischen Lehrerbildung in der Deutschdidaktik und den alltäglichen praktischen Handlungsherausforderungen von Lehrkräften im Deutschunterricht überwunden werden kann. Da nicht von einer einfachen Übertragung von fachlich-didaktischem Wissen auf das praktische Handlungsfeld ausgegangen werden kann, stellt sich für eine akademische Lehrerbildung die Frage, inwieweit die Theoriebildung und die Erkenntnisse über die akademische Lehrerbildung Eingang in den Deutschunterricht finden können. Winkler geht dieser Frage auf der Grundlage von Lehrkonzepten nach.

Gerhard Rupp verortet in seinem Beitrag „Deutschdidaktik - eine eingreifende Kultur- und kompetenzorientierte Vermittlungswissenschaft" die Disziplin in ihren Handlungsfeldern zwischen einer weitsichtigen außerschulischen Kulturwissenschaft und einer im schulischen Kontext verorteten Vermittlungswissenschaft, die enggeführt realwissenschaftlich forscht. Auf dieser Grundlage skizziert 
Rupp hilfreiche Modelle und mögliche Ansätze zur zeitgemäßen Weiterentwicklung des Fachs.

Harro Müller-Michaels Beitrag ist überschrieben mit „Denken in Ellipsen Didaktik im Spannungsfeld von Bildung und Wissenschaften“. Müller-Michaels geht davon aus, dass ein Denkrahmen der Didaktik Deutsch nur mehrdimensional zu bestimmen ist. Je nach Forschungsvorhaben, etwa Diskussion der Ziele von Bildung, Begründung der Inhalte des Unterrichts oder Leistungsprüfungen, kommen für ihn weitere Disziplinen ins Spiel. Dieses Zusammenspiel der Forschungsdimensionen kann für Müller-Michaels in der Form von Ellipsen mit je zwei Brennpunkten gefasst werden. Den unterschiedlichen leitenden Interessen wissenschaftlicher Arbeiten entsprechen dabei verschiedene Diskursformate zwischen Explikation und Normativität, die er exemplarisch erläutert.

Den Abschluss bildet die Position von Michael Krelle. In seinem Beitrag „Deutschdidaktik als (zu) gestaltende Wissenschaft?" versucht Krelle, den wissenschaftstheoretischen Ort der Deutschdidaktik näher zu umreißen. Mit Bezug auf die Arbeiten von Jakob Ossner wird dafür plädiert, die Disziplin als praktische Wissenschaft zu begreifen, mit der ein bestimmter Denkstil verbunden ist. Krelle schlägt dazu verschiedene Bezugsgrößen vor, um einen solchen gemeinsamen Denkstil zu konkretisieren und anschlussfähig zu machen.

Mit diesen zehn Positionen ist das Feld sicher nicht umfassend abgesteckt, in den vergangenen Jahrzehnten wurden verschiedene und abweichende Positionen bezogen und auch in der Gegenwart lassen sich weitere Positionierungen vorbringen. Man kann also mit Fug und Recht anzweifeln, dass die Disziplin auf dem Weg zu einem geteilt geltenden Selbstverständnis oder einem Denkrahmen ist, von dem aus die Fragestellungen des Faches grundsätzlich und allgemeingültig diskutiert werden könnten. Ein Fach aber braucht, gerade wenn ihm der Denkrahmen fehlt, die mitlaufende Diskussion über einen solchen, an deren Ende vorübergehend oder endgültig auch stehen könnte, dass es diesen einen Denkrahmen für Fachdidaktiken (zur Zeit) nicht geben kann.

\section{Literatur}

Bräuer, Christoph/Wieser, Dorothee (2015) (Hrsg.): Lehrende im Blick. Empirische Lehrerforschung in der Deutschdidaktik. Wiesbaden: Springer VS.

Bredel, Ursula/Günther, Hartmut/Klotz, Peter/Ossner, Jakob/Siebert-Ott, Gesa (2003) (Hrsg.): Didaktik der deutschen Sprache, Band 1. Paderborn: Schöningh utb.

Bremerich-Vos, Albert (1993) (Hrsg.): Handlungsfeld Deutschunterricht im Kontext. Frankfurt/Main: Diesterweg. 
Fleck, Ludwik (1999): Entstehung und Entwicklung einer wissenschaftlichen Tatsache. Einführung in die Lehre vom Denkstil und Denkkollektiv. [4. Aufl.] Frankfurt/Main: Suhrkamp. Erstmals veröffentlicht 1935.

Gölitzer, Susanne (1998): Unterrichtsbesprechungen in der Deutschlehrerausbildung. Frankfurt/Main: Peter Lang.

Gölitzer, Susanne/Roth, Jürgen (2007) (Hrsg.): Wirklichkeitssinn und Allegorese: Festschrift für Hubert Ivo zum 80. Geburtstag. Münster: Monsenstein und Vannerdat.

Hattie, John (2013): Lernen sichtbar machen. Hrsg. von Wolfgang Beywl und Klaus Zierer. Baltmannsweiler: Schneider Verlag Hohengehren.

Ivo, Hubert (1975): Handlungsfeld: Deutschunterricht. Argumente und Fragen einer praxisorientierten Wissenschaft. Frankfurt/Main: Fischer.

Ivo, Hubert (1977): Zur Wissenschaftlichkeit der Didaktik der deutschen Sprache und Literatur. Vorüberlegungen zu einer „Fachunterrichtswissenschaft“. Frankfurt/Main: Diesterweg.

Kepser, Matthis (2013): Deutschdidaktik als eingreifende Kulturwissenschaft. Ein Positionierungsversuch im wissenschaftlichen Feld. In: Didaktik Deutsch. 18. Jg. 2013. H. 34, S. 52-68.

Müller-Michaels, Harro (1972): Literaturdidaktik als normsetzende Handlungswissenschaft. In: Vogt, Jochen (Hrsg.): Literaturdidaktik. Düsseldorf: Bertelsmann-Universitätsverlag, S. 17-21.

Müller-Michaels, Harro (1980): Positionen der Deutschdidaktik seit 1949. Königstein/Taunus: Scriptor.

Ossner, Jakob (1993): Praktische Wissenschaft. In: Bremerich-Vos, Albert (Hrsg.): Handlungsfeld Deutschunterricht im Kontext. Frankfurt/Main: Diesterweg, S. $186-199$.

Ossner, Jakob (2001): Elemente eines Denkstils für didaktische Entscheidungen. In: Rosebrock, Cornelia/Fix, Martin (Hrsg.): Tumulte: Deutschdidaktik zwischen den Stühlen. Baltmannsweiler: Schneider Verlag Hohengehren, S. 17-32.

Ossner, Jakob (2006): Kompetenzen und Kompetenzmodelle im Deutschunterricht. In: Didaktik Deutsch. 12. Jg. 2006. H. 21, S. 5-19.

Rosebrock, Cornelia/Fix, Martin (2001) (Hrsg.): Tumulte: Deutschdidaktik zwischen den Stühlen. Baltmannsweiler: Schneider Verlag Hohengehren.

Schiller, Friedrich (1789): „Was heißt und zu welchem Ende studiert man Universalgeschichte?“. Reprint. Hrsg. von Volker Wahl. Jena: Verlag Dr. Bussert \& Partner.

Weniger, Erich (1952): Die Eigenständigkeit der Erziehung in Theorie und Praxis. Probleme der akademischen Lehrerbildung. Weinheim: Julius Beltz. 
Christoph Bräuer - 978-3-631-70204-8

Downloaded from PubFactory at 01/11/2019 10:39:03AM

via free access 
Christoph Bräuer

\title{
Deutschdidaktik - (k)ein Denkkollektiv ohne Denkstil? \\ German didactics - (no) thought collective without a thought style?
}

\begin{abstract}
The term thought style derived from sociology of knowledge by Ludwik Fleck is applied, on the one hand, reconstructively to the scientific cognition of didactic actions and, on the other hand, is used constructively in such a way that the scientific teaching/ learning processes become an object of German didactics as the collective coordination of showing and being shown.
\end{abstract}

\section{Deutschdidaktik - oder die Frage nach disziplinärer Identität}

Die Entwicklung der Deutschdidaktik als wissenschaftlicher Disziplin ist durch kontroverse Debatten gekennzeichnet, durch strukturelle, aber auch durch inhaltliche:

Die Kontroversen berühren zum einen die grundlegende Frage ihrer Bedeutung in und für die Ausbildung von Lehrpersonen, die Frage nach der „Eigenständigkeit der Erziehung in Theorie und Praxis“ (Weniger 1952); zum anderen die Frage ihrer Institutionalisierung an den Hochschulen und damit verbunden auch die Bezugnahme und Abgrenzung zu den etablierten Disziplinen (vgl. Winkler/Schmidt 2016) und die Klärung der wissenschaftlichen Bedeutung (vgl. Ivo 1975; 1977).

Die Kontroversen betreffen aber auch die Konstruktion der Gegenstände - ich greife nur zwei Debatten heraus, auf die ich im Weiteren noch einmal anspiele die Kontroverse um die Gestaltung des Literaturunterrichts zwischen textanalytischen und handlungs- und produktionsorientierten bzw. gesprächsförmigen Ansätzen (vgl. Winzer 2001; Menzel 2000; vgl. Nickel-Bacon 2006; Steinbrenner/ Mayer/Rank 2011) und diejenige zwischen der pragmatisch ausdifferenzierten Forderung und Förderung von Kompetenzen und der einem humanistischen Ideal verpflichteten Forderung einer umfassenden sprachlich-literarischen Bildung (vgl. Spinner 2005; Ossner 2006; Steinbrenner 2007, Wintersteiner 2007; Böhnisch 2008; Kepser 2013). Zuletzt haben es Hartmut Jonas und Marina Kreisel (2015a) 
unternommen, die Fachdidaktik Deutsch besonders in ihrer Wirkungsgeschichte und unter expliziter Berücksichtigung ihrer Erkenntnisse und Entwicklungen in der BRD und der DDR in Rückblicken und Ausblicken zu skizzieren.

Ich unterstelle, dass in diesen Debatten explizit oder implizit an der Identität der Disziplin gearbeitet wurde und wird, dass es nicht nur um Positionen oder Positionierungen innerhalb eines Denkrahmens geht, sondern um die Frage eines Denkrahmens an sich: So etwa, ob ein Kompetenzmodell einen Denkrahmen für eine Deutschdidaktik als praktischer Wissenschaft abgeben könne (Ossner 2001, 2006; Krelle in diesem Band) oder ob der Begriff der Kompetenz vielmehr einen deutschdidaktischen Denkrahmen benötige (Steinbrenner 2007). Insofern stellt sich die Frage, ob eine Debatte über die disziplinäre Identität - schon wieder oder immer noch - nötig sei und ob sie noch etwas Neues beitragen könne, nicht. Die Frage, die sich - immer wieder neu - stellt, ist diejenige der Transparenz und Reflexion im Umgang mit der disziplinären Identität und den aus ihr resultierenden disziplinären Denkzwängen und Denkverboten - mit anderen Worten, eine Auseinandersetzung mit den kollektiven Denkstilen der Disziplin.

Das sprachliche Bild des Denkrahmens mag das Feld von denkbaren Positionen besonders eindrücklich veranschaulichen: Der zweite Teil der Komposition - Rahmen - unterscheidet einerseits Flächen. Sie können von beiden Seiten bestimmt werden, als das, was deren Inhalt ausmacht (Fachinhalt, Vermittlungsmethodik, Unterricht, Lernprozesse, fachliche Ziele) oder als das, wovon er sich abgrenzt (Fachwissenschaft, Forschungsmethodik, Leben, Sozialisation und Erwerb, überfachliche Ziele). Andererseits markiert der Rahmen die Grenzlinie, die Gegenstände bzw. Ziele ein- bzw. ausschließt oder diese Prozesse selbst zum Gegenstand der Reflexion macht (Kanon, Lerninhalte und -gehalte, Fachreflexion, instrumentelle und fundamentale Ziele). Die Grenzziehung eröffnet allerdings auch erst die Möglichkeit zu Grenzverletzungen oder -überschreitungen und kann - als Schwelle verstanden - auch Übergänge abbilden. Mit der Perspektive des Übergangs kommt zugleich eine Entwicklungsperspektive in den Blick, die es erlaubt, von einer ,begrenzten Fläche' auf ein ,weites Feld' zu schauen (Abbildung 1). Ich werde versuchen zu zeigen, dass diese Perspektive das wissenschaftliche Untersuchungsfeld einer Fachdidaktik als „Wissenschaft vom fachspezifischen Lehren und Lernen innerhalb und außerhalb der Schule ${ }^{{ }^{11}}$ besonders gut zu beschreiben vermag und dass mit dieser Perspektive ein spezifischer Denkstil einhergeht.

1 So bestimmte die Konferenz der Vorsitzenden der Fachdidaktischen Fachgesellschaften (KVFF) - die Vorläuferorganisation der heutigen Gesellschaft für Fachdidaktik (GFD) - 1997 das alle Fachdidaktiken verbindende wissenschaftliche Interesse (vgl. Bayrhuber et al. 2016). 
Abb. 1: Heuristisches Raster zur Bestimmung von Denkrahmen

\begin{tabular}{|l|l|l|}
\hline $\begin{array}{l}\text { Fläche innen - positive } \\
\text { Bestimmung }\end{array}$ & $\begin{array}{l}\text { Fläche außen - negative } \\
\text { Bestimmung }\end{array}$ & $\begin{array}{l}\text { Grenze - Einschluss/ } \\
\text { Ausschluss, Übergang }\end{array}$ \\
\hline $\begin{array}{l}\text { Fachunterricht } \\
\text { (Deutsch/Stoff) }\end{array}$ & $\begin{array}{l}\text { nicht Fachwissenschaft } \\
\text { (Germanistik/Literatur/Sprache/ } \\
\text { Medien/Kultur...) }\end{array}$ & $\begin{array}{l}\text { Reflexion (der Erkenntnisse) } \\
\text { Kanon / Curriculum } \\
\text { Didaktische Kategorien }\end{array}$ \\
\hline $\begin{array}{l}\text { Vermittlung } \\
\text { (Fachmethoden) }\end{array}$ & nicht Forschungsmethoden & $\begin{array}{l}\text { Reflexion (der Erkenntniswege) } \\
\text { Didaktische Kategorien }\end{array}$ \\
\hline $\begin{array}{l}\text { Unterricht „Scola“ (schulisches } \\
\text { Handlungsfeld) }\end{array}$ & $\begin{array}{l}\text { nicht Leben/Praxis } \\
\text { „vita“ (außerschulische } \\
\text { Handlungsfelder) }\end{array}$ & $\begin{array}{l}\text { Vermittlung } \\
\text { Übergänge/Kultur }\end{array}$ \\
\hline $\begin{array}{l}\text { Bildungs- bzw. Lehr-Lern- } \\
\text { Prozesse } \\
\text { (gesteuert) }\end{array}$ & $\begin{array}{l}\text { nicht Erwerbs-/ } \\
\text { Sozialisationsprozesse } \\
\text { (ungesteuert) }\end{array}$ & $\begin{array}{l}\text { Kommunikation } \\
\text { Emergenz/Kontingenz }\end{array}$ \\
\hline $\begin{array}{l}\text { Kachliches Wissen, fachliche } \\
\text { Kompetenz- bzw. Bildungsziele }\end{array}$ & $\begin{array}{l}\text { Orientierungswissen, } \\
\text { überfachliche und allgemeine } \\
\text { Kompetenz- bzw. Bildungsziele }\end{array}$ & $\begin{array}{l}\text { instrumentelle und } \\
\text { fundamentale Lern- und } \\
\text { Bildungsziele }\end{array}$ \\
\hline
\end{tabular}

Im Zusammenspiel mit dem ersten Teil der Komposition Denkrahmen kann das Bild so entweder die Inhalte oder die Art und Weise dessen bestimmen, was in der Deutschdidaktik denkbar ist - und was nicht. Denkbar ist jedoch nicht nur ein Denkrahmen, nimmt man mehrere Denkrahmen an, so lässt das sprachliche Bild auch Schnittmengen oder Familienähnlichkeiten zu.

Versuche, Denkrahmen in diesem Sinne über ihre begriffliche Bestimmung offenzulegen, wurden von Beginn an immer wieder vorgenommen (vgl. Ivo 1975, Bremerich-Vos 1993, Rosebrock/Fix 2001). Eine Diskursanalyse, die die Debatten und deren Bestimmungsversuche bzw. deren zugrundeliegende Denkrahmen untersucht, ist für die Verständigung über die disziplinäre Identität von großem Interesse, wie es bspw. Jan Standkes (2013) Überlegungen zur Verhandlung eines literaturdidaktischen Denkrahmens auf der Grundlage der Debattenbeiträge in Didaktik Deutsch zeigt.

Gleichwohl möchte ich in diesem Beitrag einen anderen Weg vorschlagen, wenngleich mit demselben Ziel: Ich nehme den (Um-)Weg über den wissenssoziologischen Blick Flecks, der den Fokus von der Analyse der Diskurse über 
wissenschaftliche Tatsachen auf die Entstehung dieser Tatsachen selbst lenkt. Um etwas über die disziplinären Denkrahmen herauszufinden, möchte ich nicht den Denkrahmen zum Gegenstand der Untersuchung machen, sondern das - oder die - Denkkollektiv/e und dessen/deren Denkstil/e, die die in den Diskursen verhandelten Denkrahmen hervorbringen. Ich werde dazu einerseits auf schriftliche Rekonstruktionen und Analysen von Unterricht und andererseits auf (mündliche) Unterrichtstranskripte zurückgreifen. Ich nehme mit Ulla Fix (o. J.) an, dass sich das, „was wir mit Denkstil meinen und am Denkstil beobachten, ja immer sprachlich materialisiert haben muss, um wahrnehmbar, beobachtbar zu sein" (ebd., S. 1). Im Spiel der Sprache zeigt sich der Stil des Denkens. Fix verweist für eine Parallelisierung oder ein In-Bezug-Setzen von Denk- und Sprachstil auf Jürgen Schiewe, für den die Argumentation um Denkstile und Denkkollektive Hand in Hand mit der Untersuchung ihrer Sprache gehen müsse, sowohl „weil Sprache eine Verbindung zu Denken, Erkennen, Wahrnehmen“ aufweise, als auch „Institutionen als Denkkollektive stets auch ein sprachliches Korsett“ benötigten (Schiewe 1996, S. 7; zit. nach Fix o. J., S. 2).

Über einen Denkstil zu sprechen, heißt zugleich immer auch, über seinen eigenen Denkstil zu sprechen - Ludwik Fleck illustriert dies anschaulich:

Der Satz, Jan erkennt den Gegenstand C' ist unvollständig, genauso, wie der Satz, dieses Buch ist größer'. Man muss sie vervollständigen: ,Jan, als Teilnehmer an der Kultur K, oder Jan auf der Grundlage des Stils S, erkennt den Gegenstand C, dieses Buch ist größer als jenes' (Fleck 1983, S. 168; Herv. i. Original; vgl. a. Fleck 1999, S. 54).

Ich werde also bei der Rekonstruktion fremder Denkstile immer auch versuchen, den eigenen Blickpunkt offenzulegen. Und ich werde dies nicht nur in Folge der zitierten erkenntnistheoretischen Einsicht Flecks machen, sondern zum Ende argumentieren, dass diese Reflexion notwendige Grundlage für das disziplinäre Kerngeschäft ist: Die Wahrung und Wahrnehmung der Perspektivität, die Klärung der eigenen Denk-Voraus- und -Ziel-Setzungen erst ermöglicht eine Rationalität des didaktischen Denkens und Handelns. Sie sind Voraussetzung und Grundlage für „didaktisches Entscheiden“ (vgl. Ossner 2001; Bräuer 2015) und bedeutsam sowohl im Forschungs- als auch im Handlungsfeld einer praktischen Wissenschaft.

Damit ist mitnichten einem , anything goes', sondern vielmehr einem selbstbewussten offenen, rationalen Streit des didaktischen Denkens und Handelns das Wort geredet, der die eigene historisch bzw. biografisch und normativ imprägnierte Perspektive gerade auch in der empirischen Forschung reflektiert. Kollektive Denkstile sind in diesem Sinne Ausgangs- und Zielpunkt einer disziplinären Identität. Aus dem wissenssoziologischen Befund Flecks um die sich 
in den Denkkollektiven und ihren Denkstilen manifestierende soziale Bedingtheit wissenschaftlicher Entdeckungen kann nicht der erkenntnistheoretische Schluss gezogen werden, dass eine Verständigung unter verschiedenen Denkkollektiven und eine Veränderung von unterschiedlichen Denkstilen prinzipiell ausgeschlossen wäre - „die Grenzen meiner Sprache sind nicht [eo ipso] die Grenzen meiner Welt' (Wittgenstein), sie sind es kontingent oft, aber ich kann sie hintergehen." Dazu sollten Denkkollektive jedoch ihren Denkstil grundsätzlich als stilgemäßen Denkzwang reflektieren und in der Lage sein, ihn in Relation zu anderen Denkstilen zu setzen, um der Gefahr entgegenzutreten, die eigene Wahrnehmung und Deutung als unhintergehbar zu verabsolutieren:

Ein „,voraussetzungsloses Beobachten“ ist für Fleck „psychologisch ein Unding, logisch ein Spielzeug" (Fleck 1999, S. 121). In direkter Kritik an Carnaps Der logische Aufbau der Welt betont Fleck die „soziale Bedingtheit des Denkens“ und kritisiert dessen „Absolutismus der Denknormen“ und den Wunsch nach einer „Einheitswissenschaft“ (ebd., FN 3). Insofern ist die Ableitung wissenschaftlicher Überlegenheit einer (quantitativ ausgerichteten) empirischen Grundlagenforschung gegenüber einer (qualitativ ausgerichteten) anwendungsorientierten Entwicklungsforschung unzulässig. Flecks Untersuchungen verweisen gerade darauf, dass in der empirischen Grundlagenforschung denkstilgemäßes Wahrnehmen erst wissenschaftliche Tatsachen sehen und entstehen lässt.

Die Aktualität dieser Frage zeigt sich, wenn in gegenwärtigen Diskursen nun in umgekehrter Weise eine Differenzierung von grundlagenorientierten „stilreinen“ und anwendungsorientierten Forschungsprogrammen vorgenommen wird; letzteren wird grundsätzlich unterstellt, sie seien eher durch „mangelnde Stilreinheit und Heterogenität" gekennzeichnet (Brüggemann/Bromme 2006, S. 8). Diese Umkehrung erscheint vor dem Hintergrund der Fleck'schen Untersuchung eher eine normativ-wertende Verabsolutierung eines spezifischen Denkstils als die wissenssoziologisch begründete Beschreibung unterschiedlicher, sozial bedingter Denkstile zu sein. ${ }^{3}$

2 Darauf weist Jakob Ossner in einer brieflichen Reaktion auf den diesem Beitrag zugrundeliegenden Vortrag mit Nachdruck hin.

3 Brüggemann und Bromme (2006, S. 8) weisen sehr wohl darauf hin, dass dieser Grad der Heterogenität und Stilreinheit relativ sei, und insbesondere in Zeiten paradigmatischer Umbrüche auch grundlagenforschungsorientierte Ansätze durch die Verbindung unterschiedlicher theoretischer Elemente und Konstrukte aus unterschiedlichen Diskursen eine geringere Denkstilreinheit aufweisen könnten. Es bleibt aber bei einer qualitativen Differenzierung zwischen reinen und unreinen Denkstilen. 
Zunächst also meine Setzungen: Ich werde von Deutschdidaktik sprechen nicht von Fachdidaktik Deutsch und nicht von Didaktik der deutsche Sprache und Literatur - ohne den heuristischen Wert dieser Fassungen in Abrede zu stellen. Dahinter steht die Absicht, die disziplinäre Identität weder in alleiniger Abhängigkeit von einem Unterrichtsfach noch in direkter Ableitung von einer Bezugswissenschaft (Germanistik) zu denken. Und ich werde sprachlich-literarische Lehr-Lern-Prozesse - oder Bildungsprozesse - in der Sprache Deutsch als den Gegenstand dieser Deutschdidaktik unterstellen. Diese Setzung umfasst Mündlichkeit und Schriftlichkeit, Deutsch als Erst- und als Zweitsprache, institutionelle Lehr-Lern-Prozesse und außerinstitutionelle Sozialisations- und Erwerbsprozesse.

In meiner weiteren Argumentation werde ich so vorgehen, dass ich zunächst in einem ersten Schritt die Lehre vom Denkstil und Denkkollektiv skizziere und sie dann probeweise auf das Forschungsfeld Deutschdidaktik anwende, in einem zweiten Schritt auf das Handlungsfeld Deutschunterricht übertrage und in einem abschließenden Schritt überlege, was beide Perspektiven in Hinblick auf einen möglichen Denkstil bedeuten könnten und welche Implikationen aus diesen Überlegungen für eine so verstandene Disziplin Deutschdidaktik folgen könnten.

\section{Denkstil und Denkkollektiv - was mit der Denkstiltheorie zu erkennen ist}

Ludwik Fleck, von Hause aus Serologe, entwickelt seine „Lehre vom Denkstil und Denkkollektiv" in seiner Monographie Entstehung und Entwicklung einer wissenschaftlichen Tatsache, publiziert erstmals 1935. Fleck (1999) beschäftigt sich darin im ersten Teil in einer historischen Perspektive mit der Entwicklung des Syphilis-Begriffs, im zweiten Teil mit den erkenntnistheoretischen Folgerungen aus der Untersuchung einer medizinischen Fallstudie.

Im Zusammenspiel kann Fleck zeigen, wie erst die Berücksichtigung vorwissenschaftlicher, sogenannter „Präideen“ unsere Wissensvorstellungen und somit die Entstehung und Entwicklung wissenschaftlicher Tatsachen verständlich machen können. Seine Folgerung aus dieser Einsicht ist, dass die Entstehung wissenschaftlicher Tatsachen nur geschichts- und kulturabhängig denkbar ist. Am Beispiel der kollektiven Arbeit von Wassermann und seinen Mitarbeitern entwickelt Fleck einen antiindividualistischen Erkenntnisbegriff. Da für einen solchen Erkenntnisbegriff keine Begrifflichkeiten vorlagen, entwickelte Fleck ein entsprechendes Begriffsschema, das ich im Folgenden vorstellen und auf die Disziplin der Deutschdidaktik anwenden möchte. 
Fleck geht von der Annahme aus, dass Wissenschaft von Menschen kooperativ veranstaltet wird - neben den empirischen und spekulativen Überzeugungen der Individuen müssen daher vor allem die soziologischen Strukturen und die geteilt geltenden Überzeugungen der Wissenschaftler berücksichtigt werden (Schäfer/ Schnelle 1999, S. XXV) - diese geteilt geltenden Überzeugungen bezeichnet Fleck als „Denkstil“, die soziale Gruppe, die durch diesen Denkstil verbunden ist, als „Denkkollektiv“:

Definieren wir ,Denkkollektiv' als Gemeinschaft der Menschen, die im Gedankenaustausch oder in gedanklicher Wechselwirkung stehen, so besitzen wir in ihm den Träger geschichtlicher Entwicklung eines Denkgebietes, eines bestimmten Wissensbestandes und Kulturbestandes, also eines besonderen Denkstils. (Fleck 1999, S. 54 f.; Herv. i. Original)

Der Denkstil umschreibt die denkmäßigen Voraussetzungen wissenschaftlicher Erkenntnis - die in einem Kollektiv wirkenden Vorannahmen, samt der in ihnen noch weiterwirkenden Präideen.

Denkstil ist nicht nur diese oder jene Färbung der Begriffe und diese oder jene Art sie $\mathrm{zu}$ verbinden. Er ist bestimmter Denkzwang und noch mehr: die Gesamtheit geistiger Bereitschaften, das Bereitsein für solches und nicht anderes Sehen und Handeln. Die Abhängigkeit der wissenschaftlichen Tatsache vom Denkstil ist evident. (Fleck 1999, S. 85)

Ein voraussetzungsloses Beobachten gibt es für Fleck nicht, immer liegen dem Wahrnehmen denkstilgebundene Gewohnheiten und denkstilgebundene Setzungen - Fleck nennt sie „aktive Kopplungen“ - zugrunde. Nennt man mit ihm die denkstilspezifischen Festlegungen die „freien“ oder „aktiven Kopplungen“, so lässt sich die Einstellung der empirischen Wissenschaft dadurch kennzeichnen, dass sie bei einem Minimum an aktiven ein Maximum an "passiven Kopplungen“ erstrebt (Abbildung 2, unten): „Jedes Erkennen bedeutet zunächst: bei bestimmten aktiv vorgenommenen Voraussetzungen die zwangsmäßig, passiv sich ergebenden Zusammenhänge festzustellen.“ (Fleck 1999, S. 85) Empirische Deskriptionen und Objektivationen im Sinne passiver Kopplungen fußen auf kontingenten Setzungen (aktive Kopplungen) - überspitzt formuliert könnte man auch von der ,Normativität der faktischen Tatsache' sprechen.

Der Denkstil erzeugt einen Denkzwang, der nur Denkstilgemäßes sehen, Widersprüchliches dagegen nicht sehen lässt. Fleck spricht auch von einer „Harmonie der Täuschung", die widerstrebende Fakten ausblendet oder denkstilgemäß interpretiert, Gegenbeispiele als Anfangsschwierigkeiten erklärt, die im Weiteren geklärt werden könnten. 
Abb. 2: Denkzwang

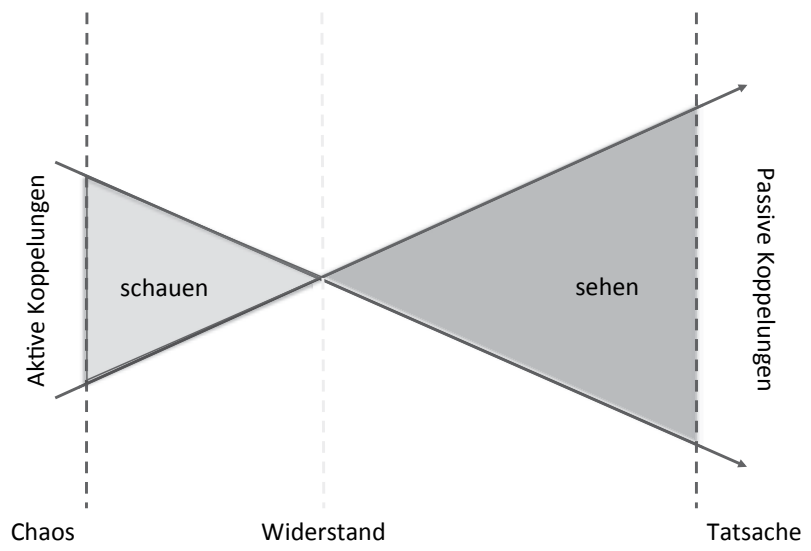

Jeder Wissenschaftler und jede Wissenschaftlerin gehört neben einem speziellen „esoterischen“ - Denkkollektiv mindestens noch einem „exoterischen“ Kollektiv der alltäglichen Lebenswelt an (vgl. Fleck 1999); in der Regel wird er aber auch Mitglied noch anderer wissenschaftlicher und nichtwissenschaftlicher Denkkollektive sein (Abbildung 3, unten) - mit Blick auf die Disziplin der Deutschdidaktik ist dies von besonderem Interesse, denn hier kommt der eigene, bildungsbiografisch schulische, berufliche und wissenschaftliche Werdegang ins Spiel. Die Auseinandersetzung mit anderen Denkkollektiven wirkt sich auf den eigenen Denkstil aus, aus dem „interkollektiven Denkverkehr“ erwachsen die Veränderungstendenzen des Denkstils (vgl. Schäfer/Schelle 1999, S. XXXIX).

So hält Fleck (1999, S. 122.) fest, dass jede empirische Entdeckung als Denkstilergänzung, Denkstilentwicklung oder Denkstilumwandlung aufgefasst werden könne.

Abb. 3: Denkkollektive

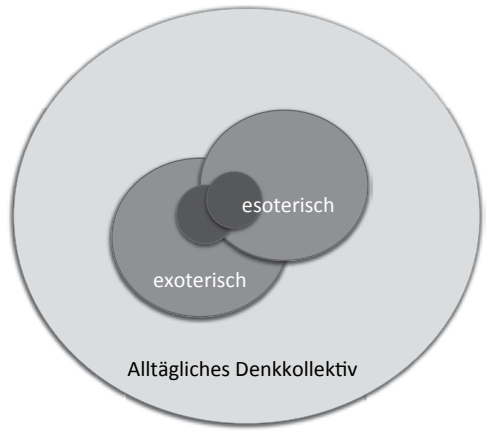


Schließlich ist ein letzter Punkt für den Austausch zwischen Wissenschaft und Praxis interessant: Fleck geht davon aus, dass sich mit der Entfernung einer Wissenschaft von der Alltagswelt auch die Anteile der aktiven Kopplungen erhöhen und in der Folge auch unmittelbar die Anzahl der passiven Kopplungen. Dies erschwert zugleich den Austausch mit der Alltagswelt, aber auch den Diskurs zwischen Denkkollektiven.

Und umgekehrt stellt er fest:

Je größer die zeitliche oder räumliche Entfernung vom esoterischen Kreis [und] je länger die Vermittlung eines Gedankens innerhalb desselben Denkkollektivs [ist], um so sicherer erscheint er. Besteht die Bindung in gedanklicher Erzogenheit aus Kinderjahren, oder gar in mehreren Generationen alter Tradition, so ist sie felsenfest. (Fleck 1999, S. 140)

\section{Denkstil in der Deutschdidaktik - Konstruktion einer Provokation}

Das Selbstverständnis eines wissenschaftlichen Kollektivs spiegelt sich in seinem Denkstil und das wissenschaftliche Kollektiv bildet einen Denkstil aus: Bildet die Deutschdidaktik ein Denkkollektiv oder mehrere, zeigt sich in der Deutschdidaktik ein ausgebildeter Denkstil oder viele? Und wäre ersteres oder letzteres wünschenswert?

Betrachtet man die Beiträge zur Identität der Disziplin über die Jahrzehnte, so stößt man auf „Tumulte“ einer nach ihrem Ort suchenden Disziplin „zwischen den Stühlen“ (Rosebrock/Fix 2001), deren Standortsuche im Blick zurück wie nach vorn ,immer wieder aufs Neue und kein Ende“ findet (Jonas/Kreisel 2015b, S. 11). Es fehlt an einem das Kollektiv einigenden Denkstil - eben dies machen sich die Herausgeber des Bandes Didaktik der deutschen Sprache unter Bezug auf Fleck zur Aufgabe:

Handbücher wachsen aus der Tiefe der Disziplin (...). Ist eine Wissenschaft weit genug entwickelt, konkurrieren verschiedene Handbücher, die dann unterschiedliche Schulen repräsentieren. (...) Ein Handbuch kann in dieser verhältnismäßig jungen Forschungsdisziplin [gemeint ist hier die Sprachdidaktik, C.B.] (...) nicht einfach den klassischen Stand zusammenfassen, sondern muss ihn zunächst einmal als solchen etablieren, in der Hoffnung, dass er von der Scientific community als wissenschaftlicher Gegenstand anerkannt wird. In gewisser Weise erwächst also das vorliegende Handbuch nicht aus der Tiefe des Faches, sondern versucht - sozusagen von oben - eine Struktur vorzuschlagen. (Bredel et al. 2003, S. 11; Herv. i. Original)

Die Herausgeber wenden die Theorie vom Denkstil also konstruktiv-disziplinbildend; das Fehlen eines Denkstils und Denkkollektivs bedeutet aber nicht das 
Fehlen von Denkstilen - ich wende die Theorie vom Denkstil exemplarisch rekonstruktiv:

Bilden „Handbücher“ gleichsam Denkstile ab, so haben Einführungen in ein Wissensgebiet oder eine wissenschaftliche Disziplin die Aufgabe einer „HineinFührung“ in Denkkollektive und Denkstile, sie üben gleichsam einen „sanften Zwang" zum gerichteten Wahrnehmen aus (Fleck 1999, S. 137):

Die Einweihung in einen Denkstil, also auch die Einführungen in eine Wissenschaft sind erkenntnistheoretisch jenen Einweihungen analog, die wir aus der Ethnologie und Kulturgeschichte kennen. Sie wirken nicht nur formell: der heilige Geist senkt sich auf den Neuling herab und bis jetzt Unsichtbares wird ihm sichtbar. Dies ist die Wirkung der Aneignung eines Denkstiles. (ebd.; Herv. i. Original)

Exemplarisch rekonstruieren lassen sich diese Einsichten an der von Heiner Willenberg herausgegebenen Einführung in einen kompetenzorientierten Deutschunterricht (2007a), die sprechenderweise den Titel Kompetenzhandbuch für den Deutschunterricht führt. Das Handbuch macht es sich zur Aufgabe, Unterricht auf der Grundlage von Kompetenzmodellen lernwirksam zu gestalten (Willenberg 2007b, S. 5):

Neu an einer solchen Fundierung ist, dass die empirisch bestätigten Kompetenzmodelle nicht nur die Leistungen der Schülerschaft in einer übersichtlichen Ordnung präsentieren, sondern dass sie sich auch in den Beobachtungen von Unterrichtsstunden wiederfinden und damit als Gütekriterien dienen können. (ebd.)

Das erste Kapitel mit dem Titel „Kompetenzen“ nimmt gleichsam über die Beschreibung des Konstrukts „Kompetenz“ die aktiven Kopplungen vor. Als „passive Kopplungen“ ergeben sich aus diesen Setzungen etwa, dass ein methodisch-strategisches Lesen zu einem Textverständnis führt und umgekehrt Textverständnis auf ein methodisch-strategisches Vorgehen verweist. NichtVerständnis ist dagegen auf ein falsches Vorgehen und/oder eine mangelnde Kompetenz zurückzuführen.

Auf dieser Grundlage - und unter diesen Vorannahmen - können die drei Fleck'schen Etappen der Entstehung einer wissenschaftlichen Tatsache rekonstruiert werden: „1. das unklare Sehen und die inadäquate erste Beobachtung; 2. Die irrationale, begriffebildende, stilumwandelnde Erfahrenheit; 3. Das entwickelte, reproduzierbare, stilgemäße Gestaltsehen." (Fleck 1999, S. 123 f.; Herv. i. Original)

Willenberg (2007c) deutet diesen Prozess - wenn auch vom Ende her gedacht an, wenn er nachzeichnet, wie die WissenschaftlerInnen aus der Leseforschung zunächst sieben Lesetätigkeiten „herausfiltern“, letztlich aber aus testpraktischen Gründen nur sechs Lesetätigkeiten weiterverfolgen. Schließlich bestätigt sich die Widerständigkeit der Tatsache: „Es hat sich als sinnvoll erwiesen, die sechs 
Tätigkeiten während des Leseprozesses in vier Kompetenzstufen einzuteilen.“ (ebd., S. 14) In Flecks Lehre ist dieser Vorgang beschrieben: „So entsteht die Tatsache: zuerst ein Widerstandsaviso im chaotischen anfänglichen Denken, dann ein bestimmter Denkzwang, schließlich eine unmittelbar wahrzunehmende Gestalt." (Fleck 1999, S. 124; Herv. i. Original).

Die Produktivität dieser „wissenschaftlichen Tatsache“ führt Willenberg im Weiteren aus, wenn er berichtet, dass es bei DESI der Schülerschaft weitgehend gelungen sei, die Informationsentnahme zu bewältigen - „bei den höheren Leseleistungen hingegen finden wir gleichsam einen Zusammenbruch der Kompetenzen. Wir werden dieses Resultat in den Unterrichtsbeobachtungen wiederfinden und daraus Vorschläge für einen intensiveren Unterricht ableiten“. (Willenberg 2007c, S. 14)

Willenberg wendet zunächst die sechs Teiltätigkeiten auf die Interpretation des Textes Nachts schlafen die Ratten doch von Wolfgang Borchert an und zeigt, wie sich dadurch ein schrittweises Verständnis des Textes aufbaut, gipfelnd in einem mentalen Modell, dessen „Motiv“ eine Interpretation der Kurzgeschichte enthält: Der Mann erkennt die auswegslose Situation des Jungen und will ihn, der glaubt, seinen toten Bruder vor den Ratten schützen zu müssen, weglotsen (vgl. ebd., S. 16).

Das Modell der Lesestufen wird nun wiederum im Sinne einer „aktiven Kopplung" auf die Beobachtung und Beschreibung von Unterricht angewendet (vgl. Willenberg 2007c, S. 16). Am Beispiel von drei Transkriptausschnitten, denen gemein ist, dass in ihnen der Borchert-Text behandelt wird, weist Willenberg nach, wie sich ein gelungener Unterricht von anderen, „weniger gelungene[n] Unterrichtsbeispiele[n] “ unterscheidet: Während Willenberg in den Äußerungen der ersten Lerngruppe das Modell der Lesestufen wiedererkennt - und diese Widerständigkeit als empirische Bestätigung begreift - , kann er in den Äußerungen der Lernenden in den beiden anderen Transkripten weder die Teiltätigkeiten noch ihre Stufenfolge erkennen.

Ich greife einen zitierten Transkriptauszug heraus (Willenberg 2007c, S. 20; Fischer 1995, S. 183): 
Transkript 1: Auszug aus einem literarischen Gespräch zu Borcherts Nachts schlafen die Ratten doch

\begin{tabular}{|c|c|c|}
\hline & & $(\ldots)$ \\
\hline 67 & $\mathrm{~L}$ & Mhm mhm Dan \\
\hline 68 & $\begin{array}{l}\text { Dan } \\
\mathrm{L}\end{array}$ & $\begin{array}{l}\text { Mit der Bombe kann ja alles im Krieg passiert sein } \\
\text { Stefan und dann Markus }\end{array}$ \\
\hline 69 & Stef. & $\begin{array}{l}\text { Die meisten denken ja das wäre mehr so in der Zeit von } \\
\text { Rittern und sowas oder }\end{array}$ \\
\hline 70 & $S$ & $\mathrm{M} \mathrm{m}$ \\
\hline 71 & $\mathrm{~L}$ & Was meinst du denn \\
\hline 72 & Stef. & Ja ich mein das ist vielleicht hat auch so mit Kríeg \\
\hline 73 & L & Mhm mhm \\
\hline 74 & $\begin{array}{l}\text { Stef. } \\
\text { L }\end{array}$ & $\begin{array}{l}\text { Und daß das also Rittér oder so sind } \\
\text { Markus }\end{array}$ \\
\hline 75 & Mark. & $\begin{array}{l}\text { Irgendwie denk ich em, was Helge gesagt hat wegen Krieg } \\
\text { das kann ja sein weil der Mann der hat ja so krumme Beine }\end{array}$ \\
\hline & & oder so. das kann ja [ . \\
\hline & Stef. & [vielleicht vom Pferd] \\
\hline
\end{tabular}

Willenberg analysiert diesen Auszug aus einem literarischen Gespräch in einer 7. Hauptschulklasse folgendermaßen:

Ein Versuch, Aspekte eines Mentalen Modells zu finden, zeigt lediglich Bruchstücke:

- Die beiden Hauptfiguren sind vorhanden.

- Schon der Ort ist komplett unklar: ist das auf dem Feld oder wo?

- Die Zeit ist nicht erkennbar, sie bleibt unwichtig.

- Die Motive sind verstümmelt: [...]. (Willenberg 2007c, S. 21)

Überprüfen wir uns als literarische Leserinnen und Leser selbst: Denkstilgemäß sehen wir in Borcherts Text als „Trümmerliteratur“ eine deutsche Stadt kurz nach Ende des zweiten Weltkrieges - wir wissen es. Wenn der Schüler Stefan eine Situation ausmalt, in denen Ritter mit krummen Beinen auf ihren Pferden in den Krieg ziehen - dann können wir darin keinen angemessenen Zugriff auf den literarischen Text von Borchert erkennen. Und es kann nicht darum gehen, dieses Rezeptionsprodukt auf irgendeine Weise in seiner Aussage umzudeuten. In der Analyse von Eva Fischer (1995) findet sich zu der von Willenberg zitierten Stelle nur ein Satz: „Erneut diskutieren sie [die Schülerinnen und Schüler, C.B.] Aspekte der Handlungslogik, ob der Junge nicht doch nur geraucht habe, zu welcher Zeit sich das alles ereigne, ob Krieg sei und Bomben gefallen seien, woher der Mann seine krummen Beine habe" (Fischer 1995, S. 191).

Interessant aber wird der zitierte Auszug, wenn man ihn nicht als finalisiertes Ergebnis einer Interpretation, sondern als Sequenz eines fortlaufenden Rezeptionsprozesses betrachtet. Dazu zeige ich den von Willenberg nicht zitierten Tran- 
skriptauszug, der dem gerade angeführten Gesprächsausschnitt unmittelbar vorausgeht (Fischer 1995, S. 183):

Transkriptauszug 2: Auszug aus einem literarischen Gespräch zu Borcherts Nachts schlafen die Ratten doch

(...)

58 Helge Es kann aber auch sein daß es vielleicht ein Soldát gewesen ist und daß er vielleicht. Äh .* ja genauso wie es die Maria gesagt hat daß er vielleicht da dieses Gebiet . äh . abge . also verbómbt hat und daß es inm jetzt leíd tut daß er da . da.

59 L $\quad$ Mhm mhm Sonja

60 Sonja Ja aber es kann doch sein was die Maria gesagt hat weil * vielleicht hat er wirklich die Bombe gelegt und daß er dann ein schlechtes Gewissen wegen dem Jungen hat daß er vielleicht jetzt Angst hat um seinen Bruder. Daß er jetzt extra auf ihn áufpaßt . Kann ja sein

(...)

64 Heike Ich glaube der Mann hat vor daß der Hase die Ersatzfigur ist für den Bruder

$65 \mathrm{~L} \quad \mathrm{Mhm} \mathrm{mhm}$

66 Heike Also daß der Hase dann den Bruder sozusagen ersetzt

Schauen wir noch einmal auf das zitierte Gespräch: Werden möglicherweise Figuren, Motive und Orte ausgehandelt? Und in einer ganz anderen Perspektive: Sprechen die Schülerinnen und Schüler nicht über den Text und ihre individuellen Lesarten dieses Textes - und arbeiten sie in diesem Gespräch nicht auf intensive und kollaborative Weise an einem mentalen Modell? Gibt es in diesem Gespräch keine „Ordnung“ oder „sehen“ wir eine, zumal unsere Ordnung nicht? Ich stelle der denkstilgemäßen Analyse des Unterrichtsbeispiels von Willenberg diejenige von Eva Fischer (1995) gegenüber und zitiere einen längeren Abschnitt:

Schon im zehnten Beitrag von über 200 dieser Stunde nennt Heike den wichtigen Interpretationsaspekt des Textes: „Ich glaub nicht also ich glaub der will eher daß er * also der Mann will glaub ich den Jungen ablenken und er will Kaninchen mitbringen damit er also nicht mehr so dran denkt damit ers irgendwann mal vergißt“" (10). Ist damit die Deutschstunde, fünf Minuten nachdem sie begann, schon zu Ende? Kann ein Gespräch danach noch weitergehen? Wirft eine Schülerin mit einer so früh ins Gespräch gebrachten Interpretation das ganze Konzept der Stunde um? Sollten doch alle Schülerinnen und Schüler in den für sie richtigen Schritten sich dem Text nähern und ihre Deutungen finden? Hinter diesen Fragen versteckt sich die gängige Befürchtung vieler Lehrerinnen und 
Lehrer, daß früh von Schülern eingebrachte interpretatorische Höhepunkte eine Stunde gefährden oder gar zerstören. ${ }^{4}$ (Fischer 1995, S. 188)

Fischer spiegelt hier einen mutmaßlichen Denkstil von Lehrkräften, sie selbst kommt bei der „Betrachtung des Gesprächsverlaufs insgesamt und der Entwicklung des Gesprächsverhaltens einzelner Schülerinnen (...) zu anderen Ergebnissen“ (Fischer 1995, S. 188; Herv. C.B.): „Ich werde zeigen, wie die Schülerinnen und Schüler einen eigenen Weg durch ihr Gespräch gehen und sich weder von Lenkungen der Lehrerin beeinträchtigen lassen noch sich gegenseitig behindern“" (ebd.).

Deutlich werden zwei unterschiedliche Wahrnehmungen einer konkreten Unterrichtssituation, deutlich werden auch unterschiedliche Zielsetzungen der beiden Didaktiker; sie führen dazu, dass unterschiedliche Sequenzen in den Blick kommen bzw. genommen werden und in diesen wiederum Unterschiedliches gesehen wird. Man könnte versucht sein, die beiden Positionen in die beiden erwähnten didaktischen Diskurse um Kompetenz (Ossner 2006) versus Bildung (Steinbrenner 2007) oder auch um eher kognitiv-textanalytische (Willenberg 2007a) versus eher handlungsorientierte bzw. gesprächsförmige Ansätze (Christ et al. 1995; Steinbrenner/Mayer/Rank 2011) in der Literaturdidaktik einzurücken.

Abgesehen von der empirischen Unproduktivität derartiger Gegenüberstellungen (vgl. Fritzsche et al. 2006) geht es mir aber um die Rekonstruktion des Denkstils. Mit Fleck können beide Wahrnehmungen der Unterrichtssituation als stilgemäßes Auflösen betrachtet werden, als „wahr“ im Denkrahmen ihres jeweiligen Denkstils:

Man kann nie sagen, derselbe Gedanke sei für A wahr und für B falsch. Gehören A und B demselben Denkkollektive an, dann ist der Gedanke für beide entweder wahr oder falsch. Gehören sie aber verschiedenen Kollektiven an, so ist es eben nicht derselbe Gedanke, da er für einen von ihnen unklar sein muß oder von ihm anders verstanden wird. Auch ist Wahrheit nicht Konvention, sondern im historischen Längsschnitt: denkgeschichtliches Ereignis, in momentanem Zusammenhange: stilgemäßer Denkzwang. (Fleck 1999, S. 131; Herv. i. Original)

Versuchsweise können wir nicht einen überlegenen und einen unterlegenen, sondern zwei verschiedene didaktische Denkstile unterstellen - und Aussagen über einen dritten, praktischen Denkstil der Lehrkräfte herauslesen; bei aller

4 Eine vergleichbare Interpretation des Umgangs mit einer zu früh zielführenden Schüleraussage findet sich bspw. auch bei Andreas Gruschka (2008). 
Unterschiedlichkeit der Unterrichtswahrnehmung setzen beide Didaktiker auf ein verändertes Denken und Handeln der Lehrkräfte, als "praktische Auswirkung“, als Anwendung des didaktisch-disziplinären Denkstils (vgl. Fleck 1999, S. 137) greifen also in den Denkstil der Lehrkräfte ein. In einer professionstheoretisch orientierten Perspektive liegt dies nahe.

Willenberg formuliert diese praktischen Auswirkungen als „Unterrichtsvorschläge“ (Willenberg 2007c, S. 22): Er verlangt die Vermittlung von Lesemethoden und deren eigenständige Anwendung, eine eingreifende Lehrkraft als Meisterleserin, die Fehlleistungen in der Anwendung der Lesemethoden korrigiert, das benötigte Wissen als Voraussetzung sicherstellt und die das mentale Modell als Ziel eines literarischen Unterrichtsgesprächs fest im Blick behält.

Fischer formuliert vorsichtiger:

Die Schülerinnen und Schüler zeigen uns deutlich, ob und wie gut sie autonom mit dem Text zurechtkommen. Wir müssen es ihnen nur zutrauen. Gerade erfahrene Lehrerinnen und Lehrer verhalten sich so, wie es sich in vielen Jahren beruflicher Praxis bewährt hat. Die eigene, vorab geleistete, fertige Interpretation eines Textes zurückzuhalten und die Schülerinnen und Schüler ihren eigenen Weg suchen und gehen zu lassen, fällt schwer. (...) Im literarischen Gespräch geht es nicht darum, dass die Lehrerin oder der Lehrer nichts tun sollte. Sich zurückzuhalten kann harte Arbeit bedeuten. (Fischer 1995, S. 198)

Ich erkenne die Einweihe in den stilgemäßen Denkzwang, der aus einem chaotischen Schauen des literarischen Gesprächs in ein lesestufen-methodisches Denken und Handeln überführt. Und ich sehe das stilgemäße Wahrnehmen einer sich selbst themenzentriert organisierenden Gesprächsentwicklung, wenn sich die Lehrkraft zurückhält und den Schülerinnen und Schülern einen Interpretationsspielraum eröffnet.

Man kann darüber hinaus aber auch das „denkgeschichtliche Ereignis“ erkennen, eine Präidee von Unterricht, in der die Lehrkraft als Experte bzw. Expertin die Schülerinnen und Schüler in das denkstilgemäße Verstehen eines literarischen Textes einführt versus eine Präidee von Unterricht, in der die Lehrkraft ebenfalls als Expertin gilt, die den Schülerinnen und Schüler diese Einweihe jedoch vorenthält.

Deutlich spielen hier in die Wahrnehmung von Unterrichtssituationen tradierte Überzeugungen hinein, die sowohl die Form von Unterricht als auch die Rolle der Lehrkraft und das Ziel von Litertur(unterricht) betreffen. Thomas Zabka (2012) hat verschiedene mutmaßliche „Analyserituale und Lehrerüberzeugungen“ skizziert, wie sie unterrichtliche „Interpretationskulturen“(vgl. Lessing-Sattari et al. 2015) ausformen dürften. Ihre Erforschung im Sinne einer Praxeologie litera- 
rischen Lernens und Verstehens steht gleichwohl noch aus, es verlangte letztlich die Rekonstruktion literarischer Denkkollektive und ihrer Denkstile, wie sie hier gefordert wird.

Ich überspitze: Deutlich wird die Finalisierung des literarischen Leseprozesses hin auf ein mentales Modell bzw. eine Deutung, verstanden als eine (literaturwissenschaftlich) angemessene Interpretation eines literarischen Textes im Unterricht (vgl. Bräuer/Rabenstein/Strauss 2017). Literaturunterricht wird bemessen an den in ihm erzeugten Produkten und weniger an den in ihm angestoßenen Prozessen. Dies beruht auf einer exoterischen Vorannahme, auf einer Präidee: Die Lehrkraft verbürgt das fachliche Wissen in seiner Fülle und Gänze, ihr Unterricht führt den Meisterschüler oder die Meisterschülerin in die Meisterlehre der Interpretation ein; durch alle Kompetenzorientierung hindurch scheinen historische Lehr-Lern-Formen, die im Nachvollzug der Meisterlehre gipfeln, sei es gleichsam, katechetisch' in Vor- und Nachrede (wie in einem Lehrervortrag), sei es gleichsam ,sokratisch' in Rede und Widerrede (wie in einem „Lehrervortrag mit verteilten Rollen“, aber auch dem Fischen nach Antworten wie beim „Rätselraten“; vgl. Ehlich/Rehbein 1986) - der Meister bzw. die Meisterin blickt aus seiner/ihrer Warte auf die Schülerinnen und Schüler, der Blick ruht auf der Lücke zwischen dem (Nicht-)Wissen der Lernenden und dem Wissen des Lehrenden. Den Lernhorizont der Novizen bildet der Standort des Experten (Abbildung 4, unten).

Dieser Befund gilt für beide Beispiele: Auch die Forderung, die Lehrkraft möge sich zurückhalten, bleibt letztlich dieser Präidee verhaftet - statt als Meisterin aufzutreten, enthält die Lehrkraft hier ihr Meisterwissen und -können den Schülerinnen und Schülern vor. Dass die Schülerinnen und Schüler sich gleichsam autonom in die eingeführten Formen literarischer Praxis enkulturieren, bleibt eine (reform)pädagogische Hoffnung. ${ }^{5}$ Wenngleich das literarische Gespräch einen besonders geeigneten Rahmen für die Begegnung mit Literatur darstellt (vgl. Härle 2004), bleibt es Lernmedium und Lerngegenstand (vgl. Zabka 2015).

5 Auch hier werden bisweilen fruchtlose, weil ideologische Debatten um Lehrer- versus Schülerorientierung geführt, die aus der Verabsolutierung unterschiedlicher Denkstile herrühren. 
Abb. 4: Der Blick auf den Lernprozess - Vermittlung als Nachvollzug

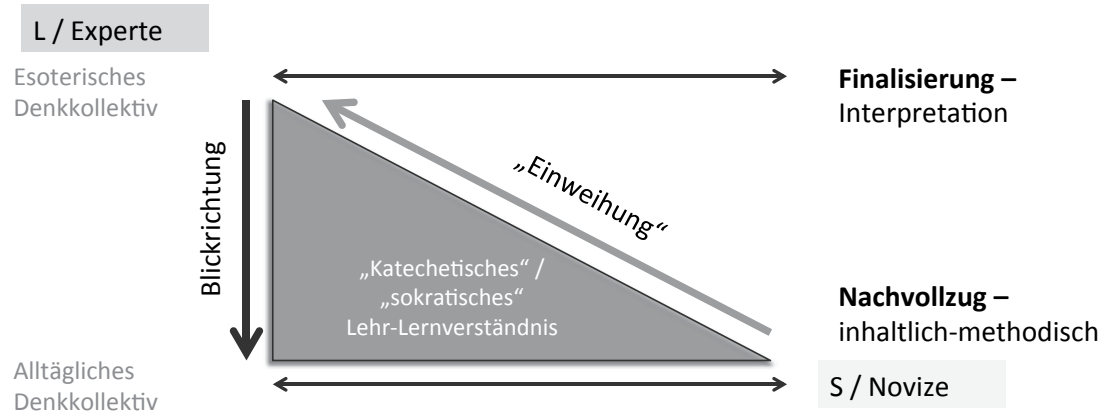

In beiden angeführten Beispielen nehmen die DidaktikerInnen keine Position „voraussetzungslosen Beobachtens“ oder reiner Deskription ein, in beiden Beispielen sind die Argumentationen normativ durchfärbt. Hier tritt die Didaktik an die Stelle der Lehrkräfte in ihrem Blick auf den Unterricht - und nicht neben sie. Mit dieser Blickrichtung einher geht jedoch der implizite und gelegentlich auch explizierte Anspruch, als Deutschdidaktikerinnen und -didaktiker die besseren Deutschlehrerinnen und -lehrer zu sein. Damit würde die Didaktik leicht zu einer Gegen-, nicht zu einer Mitspielerin. Die in den Unterrichtstranskripten selbst rekonstruierte Finalisierung zeigt sich hier wiederum - nun auf der Ebene der universitären fachdidaktischen Lehrerbildung.

Mit diesen Überlegungen habe ich - im Sinne aktiver Kopplungen - die Lehre vom Denkstil schon probeweise auf die Institution Schule und dessen Handlungsfeld Deutschunterricht übertragen - in dieser Übertragbarkeit zeigt sich im Übrigen auch für Fleck die „Fruchtbarkeit der Denkstiltheorie“ (Fleck 1999, S. 70). Im Folgenden möchte ich in sanftem Denkzwang die aus diesen Überlegungen resultierenden passiven Kopplungen ausführen.

\section{Denkstil im Unterricht - Aktive Rekonstruktion einer passiven Kopplung}

Die Didaktik hat die Praxis im Blick - und sie zielt auf die Praxis, so jedenfalls lautet ihr Ausbildungsauftrag (vgl. Ossner 1993; Ivo 1977). Es wird von ihr erwartet, Verlässliches, Wahres - Tatsachen - zu ergründen und zu vermitteln. Aber von welchem Standpunkt aus kann dieser Anspruch gelingen? Worin liegt das „Fixum“ im wissenschaftlichen Denken, in der empirischen Tatsache? Für Fleck sind wissenschaftliches Denken und wissenschaftliche Tatsachen veränderlich, 
schon „weil Denkveränderungen in veränderten Tatsachen sich offenbaren und umgekehrt grundsätzlich neue Tatsachen nur durch neues Denken auffindbar sind“ (Fleck 1999, S. 70).

Wenn das Wissen auf keinem festen Fundament ruht, sondern sich im „Getriebe der Ideen und Wahrheiten erhält [...] nur durch fortwährende Bewegung und Wechselwirkung" (ebd.), so hat es die Didaktik gleichsam mit zwei lernenden Systemen zu tun, dem der eigenen Wissenschaft und dem des fokussierten Handlungsfeldes. Die Denkstiltheorie bietet einer praktischen Wissenschaft eine Möglichkeit, beides in Relation zu setzen, um Passung und Reibung und die expliziten und die latenten Wechselwirkungen erkennen und reflektieren zu können.

Der Versuch, sich einem Denkstil der Praktiker zu nähern, setzt an Beobachtungen einer Deutschstunde 9. Klasse Gymnasium an, in der es um den Roman Tschick von Wolfgang Herrndorf geht. In diesem Roman erzählt Herrndorf von zwei 14-jährigen Außenseitern aus Berlin, die mit einem alten, geklauten Lada auf einer wunderbaren und komischen Abenteuerreise durch Ostdeutschland fahren. Die Unterrichtsstunde behandelt das Kapitel 33: In diesem Kapitel fordert Isa, ein heimatloses Mädchen, das die beiden Jungen auf einer Müllkippe treffen, Maik auf, ihr die Haare zu schneiden. Während Maik versucht, sich nicht auf Isas bloßen Oberkörper, sondern auf ihre Haare zu konzentrieren, beobachten beide, wie ein älterer Mann sich unweit selbst befriedigt - Isa verscheucht diesen Mann; im Anschluss fragt sie Maik nach dessen sexuellen Erfahrungen und Wünschen und bringt ihn so in weitere Verlegenheit (Herrndorf 2012, S. 169-172).

Die Lerngruppe sollte zunächst Textstellen herausarbeiten, in denen die Atmosphäre bzw. Stimmung beschrieben wird, anschließend Stellen finden und zitieren, in denen Maiks Gedanken und Gefühle angesprochen werden. Schließlich fordert die Lehrkraft die Schülerinnen und Schüler auf, nach "Leerstellen“ zu suchen. Zunächst werden die gefundenen Leerstellen zusammengetragen, aus einer Schülerantwort ergibt sich eine anschließende Aufgabenstellung, deren Ergebnisse schließlich vorgestellt werden (Transkript 3 und 4 unten). 
Transkript 3: Auszug aus einer Unterrichtssequenz zu Tschick - literarische Leerstelle als Ausgangspunkt für einen inneren Monolog

(...)

L gut also * ähm, habt ihr Leerstellen gefunden und wenn ja wovon ich jetzt einfach ma ausgehe * welche * Sm1

Sm Naja er sagt ja auch äh er könnte jetzt fünfhundert Seiten über seine Gefühle so schreiben

L kannst du genau mal die Textstelle angeben?

(...)

Sm <zitiert <Also mein Gehirn nahm ungeheuer Fahrt auf * und * würde schätzungsweise fünfhundert Seiten brauchen um aufzuschreiben was mir in den nächsten fünf Minuten alles durch den Kopf ging >>

L mh danke genau Leerstelle was könnte ihm denn da durch den Kopf gehen * stellt euch vor wunderbare stelle * stellt euch vor ähm ihr würdet in dem Moment äh Maik sein oder in seinen Kopf gucken können was rasen da für Gedanken rum mit den man fünfhundert Seiten füllen könnte * wär ne wunderbare Aufgabe einen inneren Monolog zu schreiben * ihr müsst keine fünfhundert Seiten füllen * aber nehmt euch kurz Zeit und notiert zwei drei Sätze oder mehr ** mindestens zwei drei Sätze oder mehr je nachdem wie schnell es euch aus der Feder fließt und was euch einfällt (...)

Je nach Provenienz unserer professionstheoretisch informierten Denkgewohnheiten sieht die literaturwissenschaftlich eingeweihte Didaktikerin eine Problematik in der Auswahl der vermeintlichen „Leerstelle“, der in kreative Methoden eingeführte Didaktiker erkennt in der Vorgehensweise der Lehrkraft „Anfangsschwierigkeiten“ bei der Arbeit mit dem inneren Monolog. Ich möchte die Gegenstandsanalyse hier nicht weiterverfolgen (vgl. dazu Bräuer/Rabenstein/ Strauss 2017), sondern versuchen, eine potentielle Blickrichtung der Lehrkraft einzunehmen:

Sie macht ihre Vorstellung von Lernen in dieser Sequenz transparent, indem sie Lernstand und Lernprogression (gleichsam die "Zone der nächsten Entwicklung“ (vgl. Wygotski 2003)) ausweist und so einen „doppelten Schüler“ (vgl. Helsper 2003) konstruiert, „von dem sie einfach einmal ausgeht“, dass er die in ihren didaktischen Rahmungen antizipierte Antwort liefert, die zugleich den Fortgang des Unterrichts sichert. Sie legt ihre Vorstellungen von der Arbeit mit literarischen Texten im Unterricht offen, wenn sie in der vom Schüler vorgeschlagenen Antwort nicht nur denkstilgemäß eine passende Antwort erkennt, sondern im gleichen Zuge auch „eine wunderbare Aufgabe für einen inneren Monolog" sieht, also zugleich einen sich geradezu zwingend ergebenden weiteren 
Verlauf eines methodisch-reflektierten literarischen Lernprozesses, der über das Schreiben der kurzen Monologe zum Inszenieren der Situation von Maik und Isa führt. Susanne Gölitzer (1998) hat einen solchen sich gleichsam von selbst ergebenden Unterrichtsverlauf als „pädagogisches Normalmodell“ bezeichnet: Fleck spricht von „größtem Denkzwang bei kleinster Denkwillkürlichkeit“ (Fleck 1999, S. 124).

Um den Unterricht entsprechend durchzuführen, muss die Lehrkraft sicherstellen, dass in der Unterrichtsinteraktion ein klar begrenzter Freiraum der Auseinandersetzung mit den Gegenständen eröffnet wird: Um zu einer Finalisierung im Unterricht zu gelangen, müssen die literarischen Rezeptionsund Verstehensprozesse gleichsam "geformt" werden, ihre raum-zeitliche Dimension eingehegt werden: Jeder Öffnung folgt sogleich eine Schließung, wie sie sich an der Aufgabenstellungen - 500 Seiten werden zu zwei bis drei kurz notierten Sätzen - und in der Ergebnissammlung - reihum wird kommentarlos vorgelesen - deutlich ablesen lässt. Der Vergleich der inneren Monologe zeigt im Übrigen, dass die Schülerinnen und Schüler gut eingeweiht sind in diesen Unterrichtsrahmen, ihre Antworten bewegen sich genau im Rahmen dieses zugestandenen raum-zeitlichen Spiels - nur in einem Fall wird dieser Denkrahmen verletzt:

Transkript 4: Auszug aus einer Unterrichtssequenz zu Tschick - Auswertung der Inneren Monologe

(...)

L so letzter Satz kommt zum Schluss * wie gesacht auf die fünfhundert Seiten wollen wir nicht komm * so Punkt Stifte aus der Hand also wir starten $\mathrm{Sm} 2$ und wir gehen reih um durch

(...)

Sm5: hat sie das gerade echt gefragt eigentlich ist sie doch total attraktiv aber * das macht man doch nicht einfach so * und was wenn Tschick jetzt kommt <lacht, einzelne in der klasse lachen auch>

Sm6: ähm das kam jetzt aber wirklich unerwartet warum hab * ich nur nein gesagt * das äääh * Scheiße verdammt ich muss ja sagen $<$ einige Schülerinnen lachen>

Sm5: <zu Sm6 < (du bist zu notgeil?)> einige beginnen zu lachen>

Sm6: ja ich hab das nicht aufgeschrieben ich hab mir das ausgedacht

$\mathrm{L}$ : lass das mal unkommentiert stehn einfach weiter tschuldigung

(...)

Sw6: (unverständlich) ähm das wäre sowieso viel zu früh wir kennen uns noch gar nicht lang und sie fragt schon * sowas denkt sie da wirklich drüber nach * nimmt sie das überhaupt ernst

(...) 
In dem Schülerkommentar von Sm5 zu Sm6, der jenseits des Unterrichtsrahmens erfolgt und folgerichtig als Rahmenverletzung von der Lehrkraft geahndet wird (vgl. Bräuer 2010, S. 152-155), fällt noch etwas auf: Wenngleich diese Äußerung als Provokation von Sm6 gelesen werden kann, schwingt in dieser Provokation ein Reflex auf den literarischen Text mit, der Maik nicht nur mit einer moralisch herausfordernden Entscheidungsfrage, sondern vor allem auch mit seinen eigenen latenten sexuellen Bedürfnissen konfrontiert (vgl. Bräuer/Rabenstein/ Strauß 2017). Diese zweite Lesart erkennt, wer die "poetologische Differenz" als Differenz „zwischen der innerfiktionalen Begründung und der außerfiktionalen Begründung eines innerfiktionalen Sachverhalts“ (Gerigk 2002, S. 17) auflöst: Dann löst sich der unbestimmte Zusammenhang zwischen der Episode mit dem Mann am Waldrand, der sich selbst befriedigt, und der Haupthandlung, in der Maik Isa die Haare schneiden soll, als Tagtraum Maiks auf, in dem zugleich seine Ängste, Bedürfnisse und Träume offengelegt werden, die ihn im Angesicht der halbnackten Isa übermannen, nicht aber sprachlich zu fassen sind. Auf eben diese rasenden Empfindungen weist die Bemerkung von Sm5 hin. Während der Disput zwischen Sm5 und Sm6 direkt auf eine Auseinandersetzung mit dem literarischen Text zielt, antworten die vorgelesenen inneren Monologe letztlich nur auf die Aufgabenstellung der Lehrkraft, deren Beantwortung sich im Übrigen selbst im weiteren Textverlauf des Romans findet. So resümiert Maik selbst, dass seine Gedanken nicht sehr spannend gewesen seien, ,ich fragte mich nämlich hauptsächlich, ob Isa das ernst gemeint hatte, und auch, ob ich das ernst gemeint hatte, als ich gesagt hatte, dass ich nicht mit ihr schlafen will, falls ich das überhaupt gesagt hatte“ (Herrndorf 2012, S. 171).

Dieser sprachlich-literarisch hochinteressante Aspekt gerät in diesem Unterricht nicht in den Blick - und kann es auch nicht: Dieser Unterricht resultiert aus zwei aktiven Setzungen, dem Ziel (besonders physischer) Aktivität und dem Ziel (schriftlicher) Produktivität von Schülerinnen und Schülern.

Ich überspitze wiederum: Die Finalisierung des Unterrichtsprozesses hin auf ein aktiv erzeugtes Produkt, verstanden als kollektives Ergebnis eines unterrichtlichen Lehr-Lern-Prozesses beruht auf einer Präidee: Die Lehrkraft verbürgt, dass jeder Schüler und jede Schülerin am Ende der Stunde etwas Gefertigtes (Gelerntes) nach Hause trägt.

Hier werden die Schülerinnen und Schüler vom Lehrer durch die Stunde und $z u$ einem Ergebnis geführt - in der gelungenen Stunde vollziehen die Schülerinnen und Schüler die methodische Inszenierung der Gegenstände wie von alleine nach. Lehrkraft und Schülerinnen und Schüler fügen sich dem Denkzwang, der 
beidem - der Entfaltung des Gegenstandes und der Entfaltung der Schülerinnen und Schüler - literaturunterrichtliche Grenzen setzt. Das unterrichtliche Vorgehen steht dabei durchaus im Einklang mit den von der Literaturdidaktik vorgeschlagenen Methoden im Literaturunterricht:

Einen (inneren) Monolog einer Figur schreiben: Diese Aufgabe ist dem Schreiben von Tagebucheinträgen verwandt; auch innere Monologe geben subjektive Erlebnisweisen von Menschen wieder. Sie sind z. B. bei Textstellen interessant, bei denen sich eine Figur in einer Entscheidungssituation befindet. (...); der Arbeitsauftrag kann auch lauten: „Stell dir vor, du bist... (Name der Figur). Schreibe auf, was du als... (Name der Figur) gerade denkst und fühlst!“ (...). (Spinner 2010, S. 226; Herv. C.B.)

Aus literaturdidaktischer Sicht dürfte unstrittig sein, dass aus dem Umstand, dass der Innere Monolog zu einer Entscheidungssituationen einer literarischen Figur eine anregende und zielführende Schreibaufgabe darstellen kann, nicht folgt, dass in jeder Entscheidungssituation ein innerer Monolog zu einer literarischen Figur geschrieben werden sollte. Aus literaturunterrichtlicher Warte kann das Vorgehen der Lehrkraft durchaus als Nachvollzug didaktischer Lehre gelten.

In Hinblick auf eine unterrichtstheoretisch informierte Beschreibung dieser Praxis ist eine Verortung im didaktischen Diskurs um Kompetenz oder Bildung, um textanalytische oder handlungs- und produktionsorientierte Verfahren unergiebig. Lehrkräfte denken und handeln in ihrem Denkrahmen denkstilgemäß sie gehören aber einem anderen Denkkollektiv an. Dies gilt es wiederum in professionstheoretisch informierter Perspektive zu berücksichtigen.

\section{Denkrahmen Deutschdidaktik - denkstilgebundene blinde Flecken und ihre Implikationen}

Es verlangte eine intensive historische Analyse, ob sich beide Denkkollektive im Rahmen einer sich verändernden Lehrerausbildung in fachlich-didaktischer Perspektive auseinander entwickelt haben - etwa in Hinblick auf das Gymnasium oder ob beide (noch) nicht zueinander gefunden haben - etwa in Hinblick auf die Volksschule bzw. Grund-, Haupt- und Realschule. Oder ob sich gleichsam gespeist aus Einflüssen des gemeinsamen alltäglichen Denkkollektivs die Denkstile in Wissenschaft und Unterricht unterschiedlich weiterentwickelt haben, und gleichsam Unterricht und literarische Gegenstandskonstruktion jeweils anders gedacht oder anders verhandelt werden.

Die Annahme, es handele sich um zwei Denkkollektive, die nur lose verknüpft sind, erklärte jedenfalls eine Anzahl von Problemen und „Tatsachen“ (im Fleck'schen Sinne). 
In meinen bisherigen Rekonstruktionen fällt eine Gemeinsamkeit auf - die Autorität des Experten und die Finalisierung des Prozesses hin auf ein Produkt, die sich in einer spezifischen Blickrichtung auf Unterricht äußert. Oder anders pointiert: Die Lernenden geraten nicht recht in den Blick, die Lerngegenstände aber recht aus dem Blick.

Eine Verständigung zwischen oder unter den (beiden) Denkkollektiven kann nur gelingen, wenn das Handlungsfeld selbst in den Blick genommen wird, wie etwa in den Arbeiten von Gölitzer (2008), Bräuer (2010) oder Reinert (2015). Auch die Integrative Deutschdidaktik (2015) von Irene Pieper und Ursula Bredel nimmt das Handlungsfeld zum Ausgangspunkt ihrer Darstellung. In ihrer Einführung rekonstruieren die Autorinnen zu Beginn Lernprozesse im Deutschunterricht auf der Grundlage von vier Transkriptauszügen. Dazu präsentieren sie zunächst zwei „gegenstands- und lernerferne Unterrichtsverläufe“, um diesen im Anschluss zwei gelungenere Alternativen gegenüberzustellen.

In den beiden als problematisch eingeführten Unterrichtsverläufen, einer Stunde zum (Recht-)Schreiben, in der es unter anderem um die v-Schreibung geht, und einer Stunde zum literarischen Lernen, in der mit der Lektüre einer Ganzschrift („Leseprojekt“) begonnen wird, arbeiten die Autorinnen einen „direktiven Lehrmodus" heraus (Bredel/Pieper 2015, S. 50):

Die Perspektiven auf den Gegenstand werden lehrerseitig gesetzt und von den Lernenden reproduziert und/oder weiterverarbeitet. Die Perspektiven der Schüler/innen auf den Gegenstand werden hingegen kaum aufgegriffen.

Diese Beobachtung ist anschlussfähig an das oben als Finalisierung bestimmte stilgemäße Denk- und Sprachhandeln der Lehrkräfte im Unterricht.

Die vorgeführten Sequenzanalysen sind für sich wie in ihrer kontrastiven Gegenüberstellung ebenso anregend wie diskussionswürdig, im Rahmen dieses Beitrags möchte ich aber nicht auf die kontrastive Analyse eingehen, sondern einen Aspekt rekonstruieren, der in beiden Unterrichtsverläufen zum Rechtschreiben auftritt, der bei den beiden Autorinnen aber nicht im Zentrum der Analyse (und Kritik) steht.

Den Anfang beider Stunden bildet eine Reihe von Worten an der Tafel, in denen jeweils der Buchstabe $<\mathrm{v}>$ auftritt: Kurve, Vase, Verlust, davon ${ }^{6}$, vielleicht, Vergangenheit, verlieren (Bredel/Pieper 2015, S. 22; S. 54).

In beiden Stunden schließt an diese Präsentation keine explizite Aufgabenstellung durch die Lehrkräfte an; Bredel und Pieper sprechen hier von einem sogenannten „stummen Impuls“ (ebd., S. 22). Rekonstruiert man die Unterrichts-

6 In der Alternativstunde ist „davon“ durch „Vater“ ersetzt worden. 
rahmung in ihrer „metonymischen Struktur“ (Herrlitz 1994, S. 13-52), nach der „die einzelnen Handlungszüge und -komponenten nicht nur in ihrer Abfolge als Teile eines Ganzen zu betrachten sind, sondern gewissermaßen auch als die an der Oberfläche sichtbaren ,Spitzen von Eisbergen' der darunter liegenden ,Massive' ganzer fachlicher und oder national geprägter Unterrichtskulturen“ (Haueis 2015, S. 166), kommen auch unterhalb der Sichtstruktur liegende Schichten „didaktischen Brauchtums“ (ebd., S. 165-168) in den Blick. Und dann lässt sich das Interaktionsmuster des „stummen Impulses“ im Unterricht präziser als „verschwiegene Aufgabenstellung" analysieren (Bräuer 2010, S. 24-25). In beiden Klassen blicken die Schülerinnen und Schüler auf die Formseite, kein Schüler und keine Schülerin beginnt etwa mit freien Assoziationen zu den Wörtern oder der Erzählung einer Reizwortgeschichte. Die verschwiegene Aufgabenstellung lautete dann in ihrer explizierten Form: Was fällt euch - auf der Schriftseite und/oder auf der Lautseite - an den sieben Wörtern der Tafelreihe auf?

Worum geht es also in dieser Aufgabenstellung in didaktischer Perspektive? Auf der Ebene der „Oberflächenstruktur“ (Oser/Patry 1990) mag es um die orthografisch korrekte Schreibung oder die orthoepisch korrekte Aussprache dieser sieben Wörter gehen, auf der Ebene der „Tiefenstruktur“, auf der die Verstehensprozesse liegen und der „Sinnfluss der Lernenden“ (vgl. Aebli 1994) verläuft, geht es um die Einsicht in die den Wörtern gemeinsamen oder sie differenzierenden Ordnungsstrukturen bzw. Relationen. In beiden Lerngruppen kommen so die Relationen zwischen Phonemen und Graphemen in den Blick.

Geht man von einer prinzipiellen Gleichberechtigung von graphischer und phonologischer Struktur aus, dann lässt sich von „Korrespondenzregeln“ sprechen, die grundsätzlich in zwei Richtungen bestehen: als Graphem-PhonemKorrespondenz und als Phonem-Graphem-Korrespondenz (Ramers 2014, S. 294). Aus dem Umstand, dass die Beziehungen in beiden Richtungen bestehen, folgt indes nicht, dass die jeweilige Perspektive irrelevant wäre, sondern vielmehr, dass die jeweilige Perspektive auszuweisen ist: Blicken wir auf die Schriftseite oder auf die Lautseite bzw. von der Lautseite auf die Schriftseite oder von der Schriftseite auf die Lautseite?

In beiden Transkriptauszügen zeigt sich nun der Wechsel der Perspektiven, ohne dass diese ausgewiesen würden: So antwortet die Schülerin Franziska im ersten Unterrichtsverlauf auf die verschwiegene Aufgabenstellung mit der Beobachtung, die Wörter fingen ,entweder mit Vogel-V an oder in der Mitte des Wortes [...] ist ein Vogel-V“ und orientiert damit auf die Schriftseite (Bredel/ Pieper 2015, S. 22); drei Wortbeiträge später äußert Mustafa: „Bei Kurve, da klingt das () so wie'n W, aber is echt ein $\mathrm{V}^{\text {“ }}$ - Mustafa betrachtet offensichtlich die 
Lautseite, von der aus er dann auf die Schriftseite blickt: „aber is echt ein V“. Der Wechsel zwischen den Perspektiven bleibt aber unmarkiert und unerklärt.

Eben dieser unmarkierte Wechsel zwischen den Perspektiven zeigt sich auch in der Alternativstunde: So führt der Beitrag einer Schülerin - ,alle Wörter haben ein V“ - als Antwort auf die verschwiegene Aufgabenstellung hier zu dem Tafelanschrieb der Lehrkraft: „alle Wörter mit v“ - betrachtet wird also die Schriftseite. Im folgenden Redebeitrag verweist die Schülerin Christin von der Schriftseite auf die Lautseite, wenn sie feststellt: „bei manchen Wörtern zum Beispiel, wie bei Kurve und Vase, da klingt es so wie ein W", kurz darauf wiederholt sie die Beobachtung nochmals und präzisiert „Kurve und Vase. Äh:, weil da klingt das V so wie’n W?“" (Bredel/Pieper 2015, S. 54-55); die Schülerin Julia ergänzt in ihrem anschließenden Redebeitrag: „Also bei Verlust, Vater, verlieren, vielleicht und Vergangenheit, hört sich das V (-) nicht so wie'n (-) V an, sondern wie'n F“" (Bredel/ Pieper 2015, S. 55).

Im Raum stehen nun zwei Relationen:

- der Konsonant /v/, der in den Wörtern Kurve und Vase verschriftet wird mit dem Konsonantenbuchstaben $<v>$, und

- der Konsonantenbuchstabe $<\mathrm{v}>$, der in fünf Wörtern der Wortreihe gesprochen wird wie der Konsonant /f/. Diesen Wörtern wird anschließend im Unterricht der Alternativstunde die Überschrift „,v' klingt wie ,f““ zugeordnet.

Entsprechend monieren Bredel und Pieper in ihrer Analyse der Gegenstandskonstruktion der Alternativstunde auch, dass unklar bliebe, „welche Vorstellung die Schülerin Julia davon hat, wie sich ein V anhören sollte" (ebd., S. 59). Darüber hinaus zeigt sich aber bei aller Unterschiedlichkeit der beiden Stunden eine Gemeinsamkeit: Die Perspektive zwischen Laut- und Schriftseite wechselt mehrfach und unmarkiert, die grundlegenden Relationen zwischen Phonemen und Graphemen werden von beiden Lehrkräften nicht geklärt, obwohl in beiden Lerngruppen die entsprechenden Beobachtungen von Schülerinnen und Schülern geäußert werden. Systematisch geordnet werden können diese Beobachtungen erst dann, wenn unter Beachtung der jeweiligen Perspektive die entsprechenden „unmarkierten Graphem-Phonem-Korrespondenzregeln“ gezeigt und erklärt werden (vgl. Ramers 2014, S. 295):

Blickt man von der Lautseite (Phoneme) auf die Schrift-Seite (Grapheme), so wird das Phonem /f/ regelmäßig mit dem Graphem $<\mathrm{f}>$ verschriftet, außer 
in den markierten Ausnahmen wie bei Vater, vielleicht, davon (idiosynkratische Schreibungen) und den Präfixen bei verlieren und Vergangenheit. ${ }^{7}$

Schaut man von der Schriftseite (Grapheme) auf die Lautseite (Phoneme), dann wird das Graphem $<w>$ regelmäßig mit dem Phonem /v/ gesprochen (bspw. das Wort "Wandern"), aber in zahlreichen Fremdwörtern, vornehmlich des Lateinischen und Französischen, wird auch das Graphem $<\mathrm{v}>$ als /v/ gesprochen (bspw. das Wort „Vase“).

Man mag Bredel und Pieper in ihrer Kritik an der Gegenstandskonstruktion des ersten Unterrichtsbeispiels nicht widersprechen, es gebe in der Stunde nichts Substantielles über die v-Schreibung zu lernen (Bredel/Pieper 2015, S. 37). Im Gegensatz dazu wird in der alternativen Stunde einiges über die v-Schreibung sichtbar. Einsichtig wird das Sichtbare aber erst dann, wenn die Blickrichtungen derart koordiniert werden, dass für alle dasselbe sichtbar wird - wenn also ausgewiesen wird, von welchem Standort aus jemand auf etwas blickt, um entweder diesen Standpunkt einnehmen oder den eigenen Standort abgrenzen zu können.

In beiden Stunden zeigt sich, dass genau das nicht geschieht; dabei verlangte der „stumme Impuls“ im Zusammenspiel mit der Wortreihe an der Tafel umso mehr nach Transparenz und klarer Strukturierung im weiteren Verlauf. Auch die Lehrkraft der alternativen zweiten Stunden nimmt nicht die Schülerperspektive auf und vollzieht sie nach, sondern unterstellt, sie entspräche ihrer eigenen Perspektive; die entsprechende Überschrift hatte die Lehrkraft schon vorbereitet. Auch hier zeigt sich wiederum eine Finalisierung in Hinblick auf das angezielte Ergebnis.

In der Bedeutung der Perspektivität liegt der zweite zentrale Befund, der in einer denkstil-informierten Betrachtung von Unterricht auffällt, wenn Schülerinnen und Schüler wie Gegenstände im Blick bleiben respektive kommen sollen. In Bezug auf eine funktionale Sprachbetrachtung weist Jakob Ossner (2015, S. 152) darauf hin, dass es in einer funktionalen Perspektive im Besonderen um ein Erklären geht, für Erklärungen jedoch konsistente Modelle vorhanden sein müssen: „Wenn eine Perspektive der Erklärungen nicht eingehalten werden kann, müssen die Perspektivwechsel für die Lernenden deutlich markiert und erklärt werden, warum man eine neue Perspektive braucht" (ebd.).

Der Ausweis der Perspektive obliegt nicht nur der Lehrkraft, auch die Schülerinnen und Schüler stehen in der Pflicht, ihre Perspektiven, soweit es ihnen möglich ist, auszuweisen. In dem Sinne sind Lehr-Lern-Prozesse Aushandlungs-

7 Sprachhistorische Erklärung können eine weitere anregende Perspektive auf diese Schreibungen bieten. 
prozesse, in dem es in ihnen darum geht, die Aufmerksamkeit der Interaktanten derart zu koordinieren, dass der jeweilige Gegenstand für die Interaktanten einsichtig werden kann.

Es geht um die Wahr(nehm)ung der Perspektivität und die Fähigkeit zur Perspektivübernahme. Beides im Blick zu haben, erschiene mir eine disziplinäre Grundtugend der Deutschdidaktik. Im Zentrum jedoch stünde die Lehrkraft, die nicht aus ihrer Warte zu den Schülerinnen und Schüler spricht und also belehrt, sondern aus der Warte der Schülerinnen und Schüler auf die Gegenstände blickt und sie so sehen lehrt, um sie in ihren sprachlich-literarischen Lernprozessen zu unterstützen - dies beinhaltet durchaus das Zeigen und Erklären möglicher Sichtweisen, die Einführung in einen Denkstil. Aber die Einführung in einen Denkstil, in ein Denkkollektiv wird nicht als Endpunkt, sondern als Ausgangspunkt verstanden - der Nachvollzug eröffnet nun wirklich die Möglichkeit zum Selbstvollzug, um nicht nur in die Welt der Literatur zu schauen, sondern die Welt der Literatur zu sehen.

Das schon zitierte Werk Lesen und Interpretieren von Horst-Jürgen Gerigk (2002) ist gerade durch die dort ausgeführten Interpretationen bspw. von Alexander Puschkins Schneesturm aufschlussreich: Mehr noch als die Ausführungen zum Interpretieren und das Reden über Interpretieren zeigen sie, was in den literarischen Texten zu entdecken ist, um es zeigen zu können (Ossner 2013, S. 47):

Schau, was ich dir zeige! [...] Dieses Zeigen ist fundamental, nach ihm erst setzt das Erklären und Begründen an. Und wenn man diesen Gedanken didaktisch wendet, so bedeutet er, dass es zumindest auch darauf ankommt, Schülerinnen und Schüler einen bestimmten Blick zu lehren, indem man ihnen diesen Blick zeigt [...]. (ebd.; Herv. i. Original)

\section{6 „Zeigen heißt: sehen lassen, zum Vorschein bringen“8 sprachlich-literarische Bildung im Fokus einer gemeinsamen Aufmerksamkeitsausrichtung}

Ich schließe mit Überlegungen zu einem Denkrahmen, der eher die zu Beginn skizzierten Übergänge denn die Felder in den Blick nimmt, im Kern aber vor allem auf einen veränderten Denkstil zielt, der an die Stelle der Perspektive auf rekonstruktive Kontingenz diejenige auf konstruktive Koordination rückt. Dazu werde ich mich sowohl auf sprachphilosophische als auch auf wahrnehmungs-

8 Martin Heidegger, „Das Wort“ (1958), in: Unterwegs zur Sprache, Gesamtausgabe, Bd. 12, hrsg. von Friedrich-Wilhelm von Herrmann, Frankfurt am Main 1985, S. 205-255, hier S. 210. Zit. nach: Wiesing 2013, S. 19. 
philosophische wie pädagogische Überlegungen stützen, um zum Schluss auf die Implikationen für die eigene Disziplin zu kommen.

Unterrichten wird heute über alle Forschungslinien hinweg als „soziale Praktik" begriffen; der Unterricht wird als ein hoch komplexes, nicht-lineares soziales Geschehen bestimmt, in dem wohl retrospektiv Wirkungen erkannt, aber nicht sicher vorausgesagt werden können (Meseth/Proske/Radtke 2012, S. 223). Die fehlende „Kausalität“ in der Vermittlung zwischen Lehren und Lernen zwingt die empirische Bildungs- und Unterrichtsforschung dazu, nun auf die „Wahrscheinlichkeit“ eines „,indirekten ,Ermöglichens' in der doppelten Bewegung von ,Geschehen-Lassen/Einschränken' um[zu]stellen“ (ebd.; siehe auch oben). Daraus leiten sich auch aktuelle Forderungen nach einer sogenannten evidenzbasierten Lehrerbildung ab (vgl. Neuweg 2015). In bildungswissenschaftlicher Perspektive wird den Interaktionsprozessen im Unterricht als eine „unhintergehbare Eigenschaft“ eine „doppelte Kontingenz“ zugeschrieben: Die Interaktanten wählen jeweils aus einer Mehrzahl von Aktions- und Reaktionsmöglichkeiten aus, aus denen sich wiederum neue Anschlussmöglichkeiten ergeben (Meseth/Proske/ Radtke 2012, S. 223 f.):

Der Unterrichtsverlauf müsse als Resultat einer dynamischen Interaktion zwischen Lehrperson und Schülern begriffen werden. Die reale Ordnung des Unterrichts emergiere in nicht vorhersehbarer Weise aus einer eigensinnigen, von unterschiedlichen Motiven getragenen ko-produktiven Auseinandersetzung aller Beteiligten mit einem Gegenstand unter variierenden institutionell-organisatorischen Gegebenheiten. (ebd., S. 224)

Die Nicht-Vorhersehbarkeit einfacher Prozess-Produkt-Zusammenhänge führt zu einer Nicht-Vorhersagbarkeit in der Sicht auf Unterricht; die Herausforderung der Nicht-Planbarkeit und Emergenz (vgl. Neuweg 2005) des Unterrichtsverlaufs stellt sich für die Didaktikerin wie den Praktiker am Schreibtisch gleichermaßen. Folgt man diesen unterrichtstheoretischen Annahmen, so formt der Unterricht durch sein kommunikatives Geschehen die fachliche Gegenstandskonstitution und -konstruktion - die ,Sache' maßgeblich. Die rekonstruierten Unterrichtssequenzen oben illustrieren dieses, Verschwinden der Gegenstände' in der unterrichtlichen Interaktion ebenso wie die zitierten Analysen in didaktischer Absicht dieses Verschwinden anprangern. Aus dem empirisch Beobachteten lässt sich jedoch nicht ohne Weiteres auf das empirisch Beobachtbare schließen:

Was sich gleichsam ,von außen' als das Problem der „doppelten Kontingenz“ darstellt, lässt sich in der Kommunikationssituation auch als Problem der „Koordination“ beschreiben (vgl. Lewis 1975): Sprachhandeln und Sprachverwendung verlangen und ermöglichen die Koordination der Interaktanten, dies gilt für den Unterricht (vgl. Prange 2011) im Allgemeinen, den Deutschunterricht im 
ganz Besonderen, indem sprachliche Koordination nicht nur ein Medium des Lernens, sondern ebenso sein Gegenstand ist. Das Problem der Koordination führt zur Ausbildung von „Konventionen“ (vgl. Lewis 1975): Sie erlauben es den Interaktanten, das Sprachhandeln im „Rahmen“ bekannter bzw. denkstilgemäß ,gesehener' Situationen nicht als kontingent, sondern als zwangsläufig oder folgerichtig wahrzunehmen (vgl. Goffman 1980; Bräuer 2010). Koordination richtet die Aufmerksamkeit auf ein gemeinsames Ziel hin aus. Mit einer themenzentrierten Aufmerksamkeitsausrichtung auf ein Ziel hin kommt nun auch die fachliche Gegenstandskonstitution und -konstruktion (wieder) in den Blick. Eine koordinierte Ausrichtung der Aufmerksamkeit auf ein gemeinsames Ziel vollzieht sich paradigmatisch in den frühen Gesten des „Zeigens" mit dem Finger (vgl. Tomasello 2014; Prange 2011, S. 26 f.) wie in den „Formaten“ des frühen Spracherwerbs (Bruner 2002). „Zeigen“ und „sehen lassen“ beschreibt nun die spezifische soziale Praktik im Unterricht, in dem es nicht nur um die Bewältigung bekannter (Konvention), sondern gerade auch um die Bewältigung neuer Situationen (Zeigen und Lernen) geht, besonders gut. So beobachtet Fleck schon 1929:

Ein erfahrener Lehrer fand, dass nur die wenigsten Schüler etwas Neues allein bemerken, wenn man sie nicht ausdrücklich darauf aufmerksam macht, und dass nur wenige es auch dann sofort sehen, wenn man es ihnen zeigt. Sie müssen es erst sehen lernen. (Fleck 2011a, S. 53)

Diese Beobachtung Flecks steht im Einklang mit seinen Überlegungen zum Sehen und Wissen: „Erkennen ist weder passive Kontemplation noch Erwerb einzig möglicher Einsicht im fertig Gegebenen“. Erkennen ist für ihn vielmehr eine soziale Praktik, „ein tätiges, lebendiges Beziehungseingehen, ein Umformen und Umgeformtwerden, kurz ein Schaffen" (Fleck 2011a, S. 54; Hervorhebung im Original).

Was bei Fleck noch „zeigen“ heißt, wird in der aktuellen Zeigeforschung als „sichtbar machen“ bezeichnet. Das gemeinsame „Schaffen“ bei Fleck, nämlich das „Erkennen“ bzw. „Sehen Lernen“, lässt sich mit der aktuellen Zeigeforschung auch als „Zeigen“ bestimmen: „Zeigen ist das Sehen-Lassen von etwas Intendiertem“ (Wiesing 2013, S. 21). Das heißt aber auch: Zeigen heißt, Sehen-lernen-Lehren'.

Zum Zeigen in diesem Sinne gehören immer wenigstens zwei, denn von Zeigen kann erst dann und nur dann gesprochen werden, „wenn der Akt des Zeigens dazu führt, dass das Gezeigte auch wirklich von jemandem gesehen wird“, so Wiesing (2013, S. 19 f.). Wiesing verdeutlicht das am Beispiel des Bildsehens:

Weil das Zeigen von etwas eine Handlung ist und weil ferner deshalb Dinge, die sichtbar sind, nicht allein deshalb, weil sie sichtbar sind, auch schon etwas zeigen, kann derjenige, dem etwas mit einem Bild oder sonst einem Zeigzeug gezeigt wird, nicht jemand sein, der nur durch bloßes Hinsehen und einfaches Betrachten sieht, was auf einem Bild sichtbar 
ist. Im Gegenteil: Wer ein Bild um seiner Sichtbarkeit willen betrachtet, verwendet das

Bild nicht als ein Mittel, mit dem jemand ihm etwas zeigen möchte. (Wiesing 2013, S. 48)

Versteht man bspw. literarisches Lernen in Analogie zur Ausbildung einer Bild- oder Filmlesefähigkeit als das „Sehen-Lernen“ literarischer Welten, dann ist mit dem Lesen eines literarischen Textes vieles sichtbar gemacht, manches Bekannte erkannt, aber noch nichts Neues gezeigt; das Zeigen verlangt die Koordination, das gemeinsame Ausrichten der Aufmerksamkeit auf das intendierte Moment; für diese Koordination braucht es mitunter didaktische „Zeigzeuge“, die bei diesem Aushandlungsprozess unterstützen - im Falle des literarischen Gespräches ist es die zentrierte mündliche Interaktion - das „wahre Gespräch“ (Härle 2004). Das Gespräch ist das kommunikative Mittel, mit dem und in dem die einzelnen Lesarten koordiniert werden. So betont Thomas Zabka (2015, S. 168), dass „literarische Gespräche“ im Literaturunterricht im Sinne einer Lernherausforderung Lernmedium und Lerngegenstand zugleich und also sowohl „verstehensfördernd“ als auch „verständigungsfördernd“ zu gestalten sind. Im Versuch, sich gegenseitig die eigenen Lesarten zu zeigen bzw. sehen zu lassen, erkennen die Interaktanten den literarischen Text, lernen ihn sehen. Darum geht es im wahren Gespräch: „Gezeigt wird etwas nur dann, wenn erstens dieses Etwas wirklich gesehen wird und wenn zweitens das Gesehene darüber hinaus auch noch etwas ist, von dem der Zeigende wollte, dass es gesehen wird." (Wiesing 2013, S. 20)

Mit diesem „Sehen-Lassen“ ist das Lernpotential des Zeigens bestimmt - es ist die sprachlich vollzogene und auf Sprache bezogene Ein-Weisung in einen Denkstil (Sprachstil). Die Sprache erlaubt es jedoch zugleich, in einen zweiten, alternativen Denkstil einzuweisen, dem man probeweise folgen kann, ohne ihn an die Stelle des ersten setzen zu müssen. Mit dieser erweiterten Lehre vom Denkstil lässt sich zeigen und erklären, was wir in einem Kippbild wahr-nehmen (etwa Kaninchen oder Ente auf der Umschlagabbildung): Wir können lernen, die jeweils andere Figur zu sehen - nie zugleich, aber im Wechsel der Perspektive. Wir werden in die Lage versetzt, (1) in einem Kippbild zwei Gestalten zu erkennen, und (2) in einem gegebenen Kontext das eine oder das andere für-wahr-zunehmen.

„Sobald wir an einem Wahrnehmungsgegenstand die Erfahrung des Aspektwechsels gemacht haben, können wir sagen, dass jenes, was wir bislang sahen, ein Aspekt des Gegenstandes war und nicht der Gegenstand selbst. Sobald an etwas mit einem Mal etwas anderes aufscheint, vollziehen sich demnach drei ineinandergreifende Modifikationen: die Verwandlung des Sehens in einen bewussten Akt, das Auseinandertreten von Wahrnehmungsgegenstand und Aspekt sowie die Neubestimmung des Gegenstands als Modifikation des Sehens und Denkens zugleich." (Lüthy 2012, S. 131) 
In diesem Sinne lässt sich Zeigen und Lernen als „Ineinanderarbeit“ in Erziehung und Unterricht verknüpfen (Prange 2005, S. 116 f.):

[Diese Ineinanderarbeit] ist nicht problemlos, aber deshalb nicht von vornherein vergeblich und illusionär. Sie ist es umso weniger, wenn Artikulation als ihr zentrales Thema gesehen und behandelt wird, gewissermaßen als die Stelle, wo Zeigen und Lernen, Vertiefung (prothesis) und Besinnung (pistis) aufeinandertreffen. Dieser Treffpunkt ist verantwortlich und maßgebend für die Differenz und Vereinigung von Zeigen und Lernen, für ihre Koordination und Synchronisierung [ ...]. Er tritt nicht besonders hervor, solange das Lernen nicht ausdrücklich thematisiert wird, sondern sich mitgängig einstellt [...]; er wird eingeebnet, wenn das Zeigen in der Weise dominant wird, dass es nur um die Logik eines gegebenen Sachverhalts geht; und er wird ausdrücklich und gewissermaßen pädagogisch entfaltet, wenn wir uns die Mühe machen, einem anderen mit Rücksicht auf seine Lage und Fassungskraft etwas so zu zeigen, dass er von dem, was wir zeigen, auch wirklich getroffen wird. (ebd.; Herv. i. Original)

Diese „Koordination von Zeigen und Lernen“, wie sie Klaus Prange (ebd.) ausführt, ist anschlussfähig an die gegenwärtig als wichtigste Merkmale für Unterrichtsqualität gehandelten Konstrukte der „kognitiven Aktivierung“ und der "konstruktiven Unterstützung" (Hasselhorn/Gold 2013, S. 247 f. und S. 249 f.). So zeichnet sich nach Marcus Hasselhorn und Andreas Gold „[g]uter Unterricht [...] dadurch aus, dass die Lerngegenstände inhaltlich klar und verständlich erklärt und erarbeitet werden, dass dabei auf die individuellen Lernvoraussetzungen Rücksicht und Bezug genommen und an die bereits vorhandenen Vorkenntnisse und Konzepte angeknüpft wird“ (ebd., S. 247). Betont wird eine „diskursive Unterrichtskultur“ (Lipowsky 2009) und „mit Verweis auf Wygotski die Rolle der fachgebundenen Interaktion mit dem kompetenten Anderen (Hasselhorn/Gold 2013, S. 247). Eine solche kognitiv aktivierende Interaktion mit einem bzw. einer kompetenten Anderen sollte sich, so lässt sich an einen weiteren von Wygotski geprägten Begriff anschließen, in der "Zone der nächsten Entwicklung“ (Wygotski 2003, S. 298) eines Lernenden bewegen:

Daß der Unterricht in dieser oder jener Weise auf das Entwicklungsniveau des Kindes abgestimmt sein muß, ist eine empirisch festgestellte und vielfach bestätigte Tatsache, die nicht zu bestreiten ist. [...] Die Bestimmung des Entwicklungsniveaus und seines Verhältnisses zu den Unterrichtsmöglichkeiten ist also eine unumstößliche, grundlegende Tatsache, die unbestritten ist und von der wir ohne weiteres ausgehen können. (ebd.)

Für Wygotski folgt daraus, dass mindestens zwei Entwicklungsniveaus des bzw. der jeweiligen Lernenden zu bestimmen sind und die Differenz zwischen dem Niveau, auf dem die Aufgaben unter Anleitung, unter Mithilfe eines bzw. einer kompetenten Anderen gelöst werden, und dem Niveau, auf dem das Kind Aufgaben selbständig zu lösen vermag, macht die Zone der nächsten Entwicklung aus (ebd., S. 300): 
Was das Kind heute mit Hilfe Erwachsener vollbringt, wird es morgen selbständig tun können. Die Zone der nächsten Entwicklung kann uns also helfen, das Morgen des Kindes, die Dynamik seiner Entwicklung zu bestimmen, nicht nur das in der Entwicklung erreichte, sondern auch das in der Reifung zu berücksichtigen. (ebd.)

Die Form dieser Hilfen sind als „Formate“ (Bruner 2002) bezeichnet und unter dem Prinzip des „Scaffolding“ (Kniffka 2012, S. 226 f.) modelliert worden. Mit diesem Bild des Lehrgerüsts verbunden ist eine vorübergehende Hilfestellung: Sobald der bzw. die Lernende in der Lage ist, eine sprachliche Handlung selbstständig auszuführen, wird das stützende Gerüst allmählich entfernt. Bruner spricht auch von einem „Übergabe“-Prinzip:

Daniel Sterns Arbeiten zur Entwicklung der wechselseitigen Rollenübernahme und Anpassung bei Interaktionen von Mutter und Kind zeigen [...], wie jeweils eine Situation geschaffen wird, in welche das Kind leicht und erfolgreich ,einsteigen' kann, und wie sich die Mutter dann nach und nach zurückzieht und die jeweiligen Rollen dem Kind übergibt, wenn dieses langsam fähig wird, sie auszuüben. (Bruner 1987, S. 51; zit. n. Kniffka 2012, S. 226 f.)

Indem das Zeigen und Sehen-Lassen eine kooperative Handlung darstellt, operationalisiert es die fachlichen Lehr-Lern-Prozesse in einem kompetenzorientierten Unterricht, dem es um Wissen und Können (Elemente und Relationen) geht, durch eine soziale Praktik.

Mit dem Bild des Zeigens und Lernens bzw. Sehen-Lassens kommt aber darüber hinaus etwas in den Blick, worauf es mir hier ankommt: Die Perspektivität. Im Bild des Zeigens sehen wir den Zeigenden, der sich neben den Lernenden stellt und gleichsam von seiner Warte aus das Sichtbare für die bzw. den Lernenden einsichtig macht, die gemeinsame Aufmerksamkeit auf einen Gegenstand koordiniert - er bzw. sie sieht auf die Gegenstände vom Standort der Lernenden, und dies ist raumzeitlich konkret zu verstehen, aus der Gegenwärtigkeit der bzw. des Lernenden und dessen, Sitz-im-Leben'.

Nimmt man diese Perspektive nicht nur ernst, sondern ein, behält man nicht nur das Ziel kollektiv im Blick, sondern auch den Lernweg der bzw. des Lernenden, das „Werden des Wissens“ (Wagenschein 1997). „Zeigzeuge“ bilden dann diejenigen „Verstehenselemente“, die sowohl das Vorwissen der Lernenden berücksichtigen als auch die fachliche Seite des zu verstehenden Konzepts, das aus der Sicht der Lernenden gedacht wird (vgl. Drollinger-Vetter 2011; o. J.). Barbara Drollinger-Vetter charakterisiert aus mathematikdidaktischer Perspektive die Qualität der „Verstehenselemente“ dadurch, dass sie sich unabhängig von den im Unterricht verwendeten Aufgaben, aber auch unabhängig von der gewählten Methode und den eingesetzten Sozialformen bestimmen lassen (ebd.). Eine di- 
daktische Disziplin hätte in dieser Perspektive ihre eigenen Gegenstände, ihre fachspezifischen „Verstehenselemente" hin auf ihre fachspezifischen Bildungsziele zu bestimmen, und tut es auch bisweilen schon (Abbildung 5, unten).

Abb. 5: Skizze eines lerner- und kompetenzorientierten Denkstils

\section{Literatur-, Medien-, Kulturwissenschaften...}

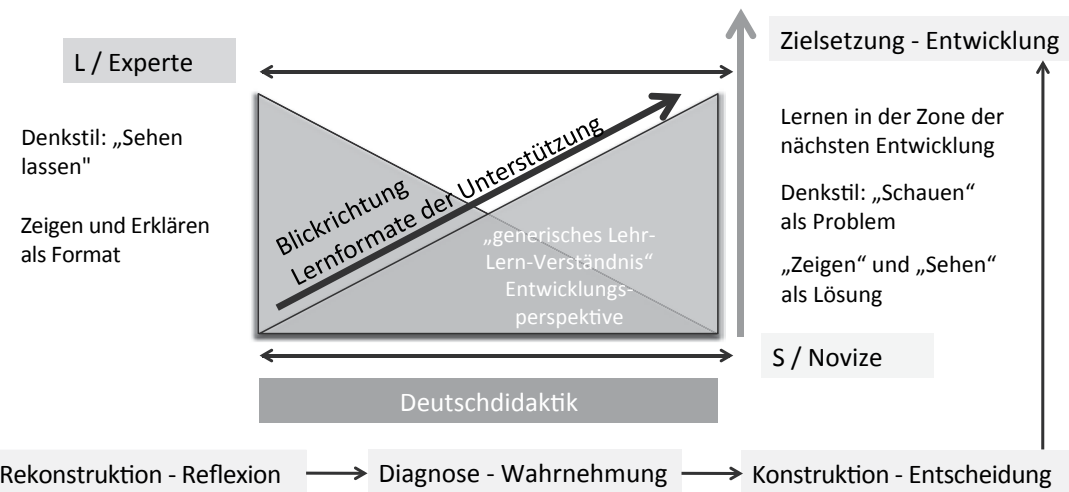

Daraus ergeben sich - in meinem Denkstil - folgende Implikationen für eine Deutschdidaktik als praktische Wissenschaft:

Die Deutschdidaktik reflektiert ihren eigenen Standort und rekonstruiert den Standort der Lehrenden - die gemeinsame Blickrichtung zielt auf das sprachlichliterarische Lernen, die Fachwissenschaften werden so zu einem Ziel-, nicht aber zum Ausgangspunkt. Auf diese Weise kann sich die Didaktik vor die Lehr-LernProzesse, aber neben die Lehrkräfte stellen: Sie liefert einen Beitrag zur Lösung praktischer Probleme, offeriert aber keine fertigen Lösungen. Ihr wissenschaftlicher Beitrag besteht in der Fundierung derjenigen Perspektiven und Verstehenselemente, die Grundlage für didaktische Entscheidungen liefern:

Dazu zähle ich:

$\Rightarrow$ Wahrnehmungskompetenz der Lehrkraft (Diagnose, Beobachtung) - Einsicht in den Verlauf von fachlichen Aneignungs-, Produktions- und Reflexionsprozesse (primäre und sekundäre Erfahrung)

$\Rightarrow$ Reflexions- und Feedbackkompetenz der Lehrenden (Denkstil, Lernkultur, Interaktionskompetenz)

$\Rightarrow$ Entscheidungskompetenz (Zielsetzung, Rationalität und Adaptivität)

$\Rightarrow$ Handlungskompetenz (Lernformate, Zeigen und Erklären, Fachlichkeit) 
Eine so verstandene Didaktik als praktische Wissenschaft berücksichtigt die doppelte Normativität einer Disziplin, die selbst für das fachliche Lehren und Lernen ausbildet. Schließlich geht es (mir hier) nicht um den „standardisierten“ Studierenden bzw. Lehrenden (vgl. Spinner 2005), sondern um eine Transparenz bzw. eine klare Sprache und Strukturierung. Und es geht um eine offene, rationale Diskussion der jeweiligen Denkstile und Denkkollektive, die nicht Setzungen, Normierungen und Konstruktionen vermeidet, sondern deren unhinterfragte oder verabsolutierende Verwendung.

\section{Literatur}

Aebli, Hans (1994): Denken. Das Ordnen des Tuns, Band II: Denkprozesse. Stuttgart: Klett-Cotta.

Bayrhuber, Horst/Abraham, Ulf/Frederking, Volker/Jank, Werner/Rothgangel, Martin/Vollmer, Helmut Johannes (Hrsg.) (2016): Auf dem Weg zu einer Allgemeinen Fachdidaktik. Allgemeine Fachdidaktik, Band 1. Münster: Waxmann.

Böhnisch, Martin (Hrsg.) (2008): Sonderheft. Beiträge zum 16. Symposion Deutschdidaktik „Kompetenzen im Deutschunterricht“. Didaktik Deutsch. 14. Jg. Sonderheft 2.

Bräuer, Christoph (2010): Kompetenz und Könnerschaft in der Leseausbildung. Theoretische und empirische Perspektiven. Weinheim/München: Juventa.

Bräuer, Christoph (2015): An der Schnittstelle professioneller Lehrkompetenz (Re-)Konstruktion von „Rationalität“ und „Adaptivität“ im Didaktischen Entscheiden. In: ders./Wieser, Dorothee (Hrsg.): Lehrende im Blick. Empirische Lehrerforschung in der Deutschdidaktik. Wiesbaden: Springer VS, S. 159-198.

Bräuer, Christoph/Rabenstein, Kerstin/Strauß, Svenja (2017): Wie findet Literaturunterricht mit textproduktiven Verfahren seine Form? Eine explorative Studie am Beispiel von Herrndorfs Tschick. Erscheint in: ZISU.

Bredel, Ursula/Günther, Hartmut/Klotz, Peter/Ossner, Jakob/Siebert-Ott, Gesa (Hrsg.) (2003): Didaktik der deutschen Sprache, Band 1. Paderborn: Schöningh utb.

Bredel, Ursula/Pieper, Irene (2015): Integrative Deutschdidaktik. Paderborn: utb. Bremerich-Vos, Albert (1993) (Hrsg.): Handlungsfeld Deutschunterricht im Kontext. Frankfurt/Main: Diesterweg.

Bruner, Jerome S. (2002): Wie das Kind sprechen lernt. [2. Auflage] Bern: Huber. Brüggemann, Anne/Bromme, Rainer (2006): Einleitung. In: dies. (Hrsg.): Entwicklung und Bewertung von anwendungsorientierter Grundlagenforschung in der Psychologie. Rundgespräche und Kolloquien. Berlin: Akademie Verlag, S. 1-10. 
Christ, Hannelore/Fischer, Eva/Fuchs, Claudia/Merkelbach, Valentin/Reuschling, Gisela (1995): „Ja aber es kann doch aber sein..... In der Schule literarische Gespräche führen. Beiträge zur Geschichte des Deutschunterrichts. Frankfurt/ Main: Peter Lang.

Drollinger-Vetter, Barbara (о. J.): „Verstehenselemente im Mathematikunterricht. https:/www.mathematik.tu-dortmund.de/ieem/cms/media/BzMU/ BzMU2009/Beitraege/alle+ModSek/Kuntz_ModSek/DROLLINGER_ Barbara_2009_Verstehenselemente.pdf. Abgerufen am 24.11.2016.

Drollinger-Vetter, Barbara (2011): Verstehenselemente und strukturelle Klarheit. Fachdidaktische Qualität der Anleitung von mathematischen Verstehensprozessen im Unterricht. Münster u. a.: Waxmann.

Ehlich, Konrad/Rehbein, Jochen (1986): Muster und Institution. Untersuchungen zur schulischen Kommunikation. Tübingen: Narr.

Fischer, Eva (1995): „...also der Mann will glaub ich den Jungen ablenken...“ „Nachts schlafen die Ratten doch“ von Wolfgang Borchert in einer 7. Hauptschulklasse. In: Christ, Hannelore et al.: „Ja aber es kann doch aber sein.... In der Schule literarische Gespräche führen. Frankfurt/Main: Peter Lang, S. 178-201.

Fix, Ulla (o. J.): Denkstil und Sprache. Die Funktion von ,Sinn-Sehen' und ,SinnBildern' für die ,Entwicklung einer wissenschaftlichen Tatsache'. http://home. uni-leipzig.de/fix/Fleck.pdf. Abgerufen am 24.11.2016.

Fleck, Ludwik (1983): Schauen, Sehen, Wissen. In: ders. (Hrsg.): Erfahrung und Tatsache. Frankfurt/Main: Suhrkamp, S. 147-174. Erstmals veröffentlicht 1947.

Fleck, Ludwik (1999): Entstehung und Entwicklung einer wissenschaftlichen Tatsache. Einführung in die Lehre vom Denkstil und Denkkollektiv. [4. Aufl.] Frankfurt/Main: Suhrkamp. Erstmals veröffentlicht 1935.

Fleck, Ludwik (2011): Zur Krise der „Wirklichkeit“. In: ders. (Hrsg.): Denkstile und Tatsachen. Gesammelte Schriften und Zeugnisse. Frankfurt/Main: Suhrkamp, S. 52-69. Erstmals veröffentlicht 1929.

Fritzsche, Joachim/Krempelmann, Anita/Tosun, Claudia/Zaborowski, Katrin (2006): Literaturunterricht kontrastiv. Der handlungs- und produktionsorientierte Literaturunterricht auf dem Prüfstand. Baltmannsweiler: Schneider Verlag Hohengehren.

Gerigk, Horst-Jürgen (2002): Lesen und Interpretieren. Göttingen: Vandenhoeck und Ruprecht.

Goffmann, Erving (1980): Rahmen-Analyse. Ein Versuch über die Organisation von Alltagserfahrungen. Frankfurt/Main: suhrkamp.

Gölitzer, Susanne (1998): Unterrichtsbesprechungen in der Deutschlehrerausbildung. Frankfurt/Main: Peter Lang. 
Gölitzer, Susanne (2008): Wozu Literatur lesen? Der Beitrag des Literaturunterrichts zur literarischen Sozialisation von Hauptschülerinnen und Hauptschülern. opus.ph-heidelberg.de/frontdoor/index/docld/24. Abgerufen am 24.11.2016.

Gruschka, Andreas (2008): An den Grenzen des Unterrichts. Pädagogische Fallanthologie, Band 10. Opladen: Barbara Budrich.

Härle, Gerhard (2004): Literarische Gespräche im Unterricht. Versuch einer Positionsbestimmung. In: ders./Rank, Bernhard (Hrsg.): Wege zum Lesen und zur Literatur. Baltmannsweiler: Schneider Verlag Hohengehren, S. 137-168.

Hasselhorn, Marcus/Gold, Andreas (2013): Pädagogische Psychologie. Erfolgreiches Lernen und Lehren. [3. Auflage] Stuttgart: Kohlhammer.

Kniffka, Gabriele (2012): Scaffolding - Möglichkeiten, im Fachunterricht sprachliche Kompetenzen zu vermitteln. In: Michalak, Magdalena/Kuchenreuther, Michaela (Hrsg.): Grundlagen der Sprachdidaktik Deutsch als Zweitsprache. Baltmannsweiler: Schneider Verlag Hohengehren, S. 221-237.

Lewis, David (1975): Konventionen. Eine sprachphilosophische Abhandlung. Berlin: de Gruyter.

Lipowsky, Frank (2009): Unterricht. In: Wild, Elke/Möller, Jens (Hrsg.): Pädagogische Psychologie. Heidelberg: Springer, S. 73-101.

Lüthy, Michael (2012): Das Medium der ästhetischen Erfahrung. Wittgensteins Aspektbegriff, exemplifiziert an Pollocks Malerei. In: Koch, Gertrud/Maar, Kirsten/McGovern, Fiona (Hrsg.): Imaginäre Medialität/immaterielle Medien. Paderborn: Wilhelm Fink, S. 125-142.

Haueis, Eduard (2015): „Brauchtum“ im Deutschunterricht - ein brachliegendes Feld der fachdidaktischen Forschung. In: Jonas, Hartmut/Kreisel, Marina (Hrsg.): Fachdidaktik Deutsch - Rückblicke und Ausblicke. Frankfurt/Main: Peter Lang, S. 165-181.

Helsper, Werner (2003): Ungewissheit im Lehrerhandeln als Aufgabe der Lehrerbildung. In: ders./Hörster, Reinhard/Kade, Jochen (Hrsg.): Ungewissheit. Pädagogische Felder im Modernisierungsprozess. Weilerswist: Velbrück Wissenschaft, S. 142-161.

Herrlitz, Wolfgang (1994): „Spitzen der Eisberge.“ Vorbemerkungen zu einer vergleichenden Analyse metonymischer Strukturen im Unterricht der Standardsprache. In: Haueis, Eduard (Hrsg.): Muttersprachlicher Unterricht an Europas Schulen. OBST. Jg. 1994. H.48, S. 13-52.

Herrndorf, Wolfgang (2012): Tschick. Roman. [15. Aufl.] Reinbek bei Hamburg: Rowohlt Taschenbuch Verlag.

Ivo, Hubert (1975): Handlungsfeld: Deutschunterricht. Argumente und Fragen einer praxisorientierten Wissenschaft. Frankfurt/Main: Fischer. 
Ivo, Hubert (1977): Zur Wissenschaftlichkeit der Didaktik der deutschen Sprache und Literatur. Vorüberlegungen zu einer „Fachunterrichtswissenschaft“. Frankfurt/Main: Diesterweg.

Jonas, Hartmut/Kreisel, Marina (Hrsg.) (2015a): Fachdidaktik Deutsch - Rückblicke und Ausblicke. Frankfurt/Main: Peter Lang.

Jonas, Hartmut/Kreisel, Marina (2015b): Immer wieder aufs Neue und kein Ende: Blick zurück und nach vorn. In: dies. (Hrsg.): Fachdidaktik Deutsch - Rückblicke und Ausblicke. Frankfurt/Main: Peter Lang, S. 11-31.

Kepser, Matthis (2013): Deutschdidaktik als eingreifende Kulturwissenschaft. Ein Positionierungsversuch im wissenschaftlichen Feld. In: Didaktik Deutsch. 18. Jg. 2013. H. 34, S. 52-68.

Lessing-Sattari, Marie/Löhden, Maike/Meissner, Almuth/Wieser, Dorothee (2015) (Hrsg.): Interpretationskulturen. Literaturdidaktik und Literaturwissenschaft im Dialog über Theorie und Praxis des Interpretierens. Frankfurt/ Main: Peter Lang.

Menzel, Wolfgang (2000): Handlungsorientierter Literaturunterricht. Praxis Deutsch. Sonderheft. Seelze: Friedrich.

Meseth, Wolfgang/Proske, Matthias/Radtke, Frank-Olaf (2012): Kontrolliertes Laissez-faire. Auf dem Weg zu einer kontingenzgewärtigen Unterrichtstheorie. In: Zeitschrift für Pädagogik. 58 Jg., H. 2, S. 223-241.

Nickel-Bacon, Irmgard (2006): Positionen der Literaturdidaktik - Methoden des Literaturunterrichts. Ein heuristischer Explikationsversuch für die empirische Grundlagenforschung. In: Groeben, Norbert/Hurrelmann, Bettina (Hrsg.): Empirische Unterrichtsforschung in der Literatur- und Lesedidaktik. Ein Weiterbildungsprogramm. Weinheim und München: Juventa, S. 95-114.

Neuweg, Georg Hans (2005): Emergenzbedingungen pädagogischer Könnerschaft. In: Heid, Helmut/Harteis, Christian (Hrsg.): Verwertbarkeit. Ein Qualitätskriterium (erziehungs-)wissenschaftlichen Wissens? Wiesbaden: VS Verlag für Sozialwissenschaften, S. 205-228.

Neuweg, Georg Hans (2015): Evidenzbasierte Lehrerbildung. In: Journal für Lehrerinnenbildung, Jg. 2015. H. 4, S. 54-58.

Oser, Fritz/Patry, Jean-Luc 1990: Choreographien unterrichtlichen Lernens. Basismodelle des Unterrichts. Freiburg: Pädagogisches Institut (Berichte zur Erziehungswissenschaft Nr. 89).

Ossner, Jakob (1993): Praktische Wissenschaft. In: Bremerich-Vos, Albert (Hrsg.): Handlungsfeld Deutschunterricht im Kontext. Frankfurt/Main: Diesterweg, S. 186-199. 
Ossner, Jakob (2001): Elemente eines Denkstils für didaktische Entscheidungen. In: Rosebrock, Cornelia/Fix, Martin (Hrsg.): Tumulte: Deutschdidaktik zwischen den Stühlen. Baltmannsweiler: Schneider Verlag Hohengehren, S. 17-32.

Ossner, Jakob (2006): Kompetenzen und Kompetenzmodelle im Deutschunterricht. In: Didaktik Deutsch. 12. Jg. 2006. H. 21, S. 5-19.

Ossner, Jakob (2013): Erklären und Zeigen. In: Didaktik Deutsch. 18. Jg. 2013. H. 34, S. 37-51.

Ossner, Jakob (2015): Funktionaler Grammatikunterricht und funktionale Grammatik. Geschichtliche Lehren und gegenwärtige Praxis. In: Jonas, Hartmut/ Kreisel, Marina (Hrsg.): Fachdidaktik Deutsch - Rückblicke und Ausblicke. Frankfurt/Main: Peter Lang, S. 141-163.

Prange, Klaus (2005): Die Zeigestruktur der Erziehung. Grundriss der Operativen Pädagogik. Paderborn: Schöningh.

Prange, Klaus (2011): Zeigen - Lernen - Erziehen. In: ders. (Hrsg.): Zeigen Lernen - Erziehen. Jena: edition Paideia, S. 21-61.

Ramers, Karlheinz (2014): Graphetik, Graphematik, Orthografie. In: Ossner, Jakob/Zinsmeister, Heike (Hrsg.): Sprachwissenschaft für das Lehramt. Paderborn: Schöningh, S. 283-323.

Reinert, Johannes (2015): Unterrichtliches Brauchtum und Kommunikationsorientierung: Spannungen bei der unterrichtlichen Arbeit mit schulischen Textformen am Beispiel der Inhaltsangabe. In: Bräuer, Christoph/Wieser, Dorothee (Hrsg.): Lehrende im Blick. Empirische Lehrerforschung in der Deutschdidaktik. Wiesbaden: Springer VS, S. 227-251.

Rosebrock, Cornelia/Fix, Martin (2001) (Hrsg.): Tumulte: Deutschdidaktik zwischen den Stühlen. Baltmannsweiler: Schneider Verlag Hohengehren.

Schäfer, Lothar/Schnelle Thomas (1999): Ludwik Flecks Begründung der soziologischen Betrachtungsweise in der Wissenschaftstheorie. In: Fleck, Ludwik: Entstehung und Entwicklung einer wissenschaftlichen Tatsache. Einführung in die Lehre vom Denkstil und Denkkollektiv. [4. Aufl.] Frankfurt/Main: Suhrkamp, S. VII-XLIX.

Schiewe, Jürgen (1996): Sprachenwandel - Funktionswandel - Austausch der Denkstile. Die Universität Freiburg zwischen Latein und Deutsch. Freiburg: Niemeyer.

Standke, Jan (2013): Das Schwierige (in) der literarischen Bildung. Kontroversentheoretische Überlegungen zur Verhandlung eines literaturdidaktischen ,Denkrahmens. In: Dawidowski, Christian (Hrsg.): Bildung durch Dichtung/ Literarische Bildung: Bildungsdiskurse literaturvermittelnder Institutionen um 1900 und um 2000. Frankfurt a. M.: Peter Lang, S. 281-301. 
Steinbrenner, Marcus (2007): Sprache denken. Eine Kritik an Jakob Ossners Kompetenzmodell. In: Didaktik Deutsch. 13. Jg. 2007. H. 23, S. 5-14.

Steinbrenner, Marcus/Mayer, Johannes/Rank, Bernhard (2011) (Hrsg.): „Seit ein Gespräch wir sind und hören voneinander". Das Heidelberger Modell des Literarischen Unterrichtsgesprächs in Theorie und Praxis. Baltmannsweiler: Schneider Verlag Hohengehren.

Spinner, Kaspar H. (2005): Der standardisierte Schüler. In: Didaktik Deutsch. 11. Jg., H. 18, S. 4-13.

Spinner, Kaspar H. (2010): Methoden des Literaturunterrichts. In: Kämper-van den Boogaart, Michael/Spinner, Kaspar H. (Hrsg.): Lese- und Literaturunterricht, Teil 2. Baltmannsweiler: Schneider Verlag Hohengehren, S. 190-242.

Tomasello, Michael (2014): Die Ursprünge der menschlichen Kommunikation. [3. Auflage] Frankfurt/Main: Suhrkamp.

Wagenschein, Martin (1997): Verstehen lehren. [11. Aufl.] Weinheim/Basel: Beltz. Weniger, Erich (1952): Die Eigenständigkeit der Erziehung in Theorie und Praxis. Probleme der akademischen Lehrerbildung. Weinheim: Julius Beltz.

Wiesing, Lambert (2013): Sehen lassen. Die Praxis des Zeigens. [2. Aufl.] Frankfurt/Main: Suhrkamp.

Willenberg, Heiner (Hrsg.) (2007a): Kompetenzhandbuch für den Deutschunterricht. Baltmannsweiler: Schneider Verlag Hohengehren.

Willenberg, Heiner (2007b): Einleitung. In: ders. (Hrsg.): Kompetenzhandbuch für den Deutschunterricht. Baltmannsweiler: Schneider Verlag Hohengehren, S. 5-6.

Willenberg, Heiner (2007c): Lesestufen - Die Leseprozesstheorie. In: ders. (Hrsg.): Kompetenzhandbuch für den Deutschunterricht. Baltmannsweiler: Schneider Verlag Hohengehren, S. 11-23.

Winkler, Iris/Schmidt, Frederike (2016) (Hrsg.): Interdisziplinäre Forschung in der Deutschdidaktik. „Fremde Schwestern“ im Dialog. Frankfurt/Main: Peter Lang.

Wintersteiner, Werner (2007): Die Innenwelt der Außenwelt der Innenwelt. Deutschdidaktik im Sog gesellschaftlicher Interessen. In: Didaktik Deutsch. Jg. 2007. H. 22, S. 51-70.

Winzer, Hans-Joachim (2001): Handlungs- und produktionsorientierter Literaturunterricht: Möglichkeiten und Grenzen. Eine Bilanz. Oldenburg: Univ., Didaktisches Zentrum.

Wygotski, Lew (2003): Unterricht und geistige Entwicklung im Schulalter. In: Lompscher, Joachim (Hrsg.): Lew Vygotskij - Ausgewählte Schriften - Band II. Berlin: Lehmanns Media-LOB.de, S. 287-306. 
Zabka, Thomas (2012): Analyserituale und Lehrerüberzeugungen. Theoretische Untersuchung vermuteter Zusammenhänge. In: Irene Pieper/Dorothee Wieser (Hrsg.): Fachliches Wissen und literarisches Verstehen. Studien zu einer brisanten Relation. Frankfurt/Main: Peter Lang, S. 35-52.

Zabka, Thomas (2015): Konversation oder Interpretation? Überlegungen zum Gespräch im Literaturunterricht. In: Leseräume. Zeitschrift für Literalität in Schule und Forschung. 2. Jahrgang 2015/Heft 2, S. 169-187. http://leseräume. de/wp-content/uploads/2015/10/lr-2015-1-zabka.pdf. Abgerufen am 14.11.16. 
Juliane Köster

\title{
Die dilemmatische Disziplin - Deutschdidaktik zwischen Eklektizismus und Partialisierung ${ }^{1}$ The dilemmatic discipline - German didactics between eclecticism and partialisation
}

\begin{abstract}
The paper addresses two means of responding to the lack of unity in the discipline: Firstly, the growing division into numerous sub-groups with very differing thought styles; and secondly, the attempt to construct unity with the aid of transdisciplinary umbrella concepts. The author then advocates being bold enough to call it a science of profession in analogy to law and medicine.
\end{abstract}

Im September 2000 wurden auf dem Freiburger Symposion die - alten - Fragen nach den Koordinaten der Disziplin verhandelt. Das Thema des Symposions hieß Grenzen überschreiten. Es ging um nicht weniger als die „Deutschdidaktik der Zukunft“ und um die Bedeutung von „Fachstrukturen im Rahmen von Interdisziplinarität“. ${ }^{2}$ Thomas Zabka (2002) hat den aus Sektion 1 hervorgegangenen Tagungsband rezensiert und fragt, ob die „Veteranen und Vordenker der Deutschdidaktik“ denn „wirklich etwas paradigmatisch Neues beigetragen haben zu diesen Debatten, die geführt werden, seit es die Disziplin gibt“. Es sind die Fragen nach dem Selbstverständnis der Disziplin, nach der theoretischen Modellierung der eigenen Forschung und nach der Bedeutsamkeit für den professionellen Alltag des Deutschunterrichts (vgl. Tenorth 2012). Schwierig sind diese Fragen, weil ihre Beantwortung davon abhängt, wie auf die offensichtliche Breite und Vielfalt sowohl des Schulfachs als auch der wissenschaftlichen Disziplin reagiert wird.

Um die Fülle der Themen und Domänen zu bewältigen, lassen sich aktuell zwei große strategische Trends unterscheiden: (1) Der Wunsch nach Unterscheidung und Abgrenzung und (2) die Orientierung an der Einheit und Vernetzung des Fachs. Das erste führt tendenziell zur Partialisierung, das zweite birgt die Gefahr

1 Für kritisch-konstruktive Kommentare danke ich Stefan Matuschek, Jakob Ossner, Dorothee Wieser und Lambert Wiesing sehr herzlich.

2 13. Symposion Deutschdidaktik, Freiburg i. Br., September 2000: Themenübersicht: Plenum, Sektionen, Workshop, Postermarkt (2000): In: Didaktik Deutsch. 5. Jg. H. 9, S. 58-69. 
des Eklektizismus in sich. Beide Strategien sind Versuche, der Heterogenität der Domäne in ihren Bereichen und Gegenständen gerecht zu werden. Um es vorwegzunehmen: Beide Strategien (die zugleich auch Bestrebungen sind) sind in einem gewissen Grad unvermeidbar, aber sie führen in ein Dilemma. Insofern ist von einer dilemmatischen Disziplin zu sprechen. Zugespitzt formuliert: Zwischen Partialisierung und Eklektizismus wird das Handlungsfeld des Deutschunterrichts zum blinden Fleck. Aus dem Blickfeld geraten seine Inhalte und Gegenstände, die Tauglichkeit von Bildungsstandards und Kompetenzerwartungen, die Lernwirksamkeit von Lernaufgaben, die Leistungsfähigkeit von Prüfungsaufgaben und Operatoren etc.

\section{Partialisierung}

\subsection{Worin zeigt sich Partialisierung?}

Sprechen wir zunächst über Partialisierung, über die Zerlegung eines Fachs bzw. einer Domäne in immer kleinere, immer spezifischere Segmente und Segmentteile. Dem Schulfach Deutsch eingeschrieben ist die Unterteilung in Lernbereiche. Ursprünglich war es die Trennung von Sprach- und Literaturunterricht, deutlich erkennbar an der Trennung von Sprach- und Lesebuch. Ab den 1980er Jahren dann die Unterscheidung von schriftlichem und mündlichem Sprachgebrauch, Umgang mit Texten und Sprachreflexion und in den 2000er Jahren die Etablierung der Mündlichkeit als vierten Lern- bzw. Kompetenzbereich. In den Bildungsstandards für die Allgemeine Hochschulreife haben wir es mit fünf Kompetenzbereichen zu tun - mit drei prozessbezogenen und zwei domänenspezifischen -, die ihrerseits in Unterbereiche gegliedert sind, die mindestens teilweise nach Eigenständigkeit streben, wie z. B. Film und Theater. Blickt man auf die Bildungsstandards von der Primarstufe bis zur Allgemeinen Hochschulreife, dann werden die zentralen Bildungsziele in 250 Einzelkompetenzen zerlegt. Bis zum ersten Schulabschluss hat man es mit etwa 150 Standards zu tun.

Was die wissenschaftliche Deutschdidaktik betrifft, so gehen die Sektionen des SDD auf die Unterteilungen des Deutschunterrichts zurück, haben sich aber im Lauf der Jahre immer weiter ausdifferenziert, sodass wir es bei den letzten Symposien mit 15 oder 16 Sektionen zu tun hatten. ${ }^{3}$

$\mathrm{Zu}$ dieser inhaltlichen Ausdifferenzierung traten vornehmlich ab 2002 Aufspaltungen in der Forschung. Aktuell handelt es sich um die Konfrontation von

3 Demgegenüber sind für das SDD 2016 nur noch 12 Sektionen angekündigt. 
HermeneutikerInnen und EmpirikerInnen, unter diesen wiederum um die Separierung der quantitativ Forschenden von den qualitativ Forschenden und die Qualitativen lassen sich in VertreterInnen der Objektiven Hermeneutik, der Ethnographie oder der Dokumentarischen Methode etc. unterscheiden. Tendenziell spiegelt sich diese Aufspaltung auch in der Unterscheidung zwischen (empirischen) Forschungsbeiträgen und Berichten in der Zeitschrift Didaktik Deutsch.

\subsection{Was macht Partialisierung problematisch?}

Problematisch werden derartige Aufspaltungen unter drei Bedingungen: (1) Wenn die Vertreter der Segmente zu einander bekämpfenden Gegnern werden; (2) wenn eine oder mehrere Parteien als nicht satisfaktionsfähig betrachtet werden und (3) - in Bezug auf empirische Verfahren - wenn im Zuge bestimmter Methoden und Verfahren sowohl der fachliche Gegenstand als auch die Operationen der ProbandInnen einer Partialisierung unterzogen werden, die den fachlichen Gegenstand entstellt und die kognitive Tätigkeit der ProbandInnen nur noch als isolierte Facette erkennen lässt. Oder anders formuliert: Wenn die auf der Basis starker Zerlegung erzielten Ergebnisse nur noch für die Forschung, nicht aber für den professionellen Alltag des Deutschunterrichts bedeutsam sind.

Auf die Partialisierungen im Methodischen kommt es vor allem deshalb an, weil sie nicht nur für den Bezug zum alltäglichen Deutschunterricht Konsequenzen haben, sondern auch für den disziplinären Status wissenschaftlicher Deutschdidaktik.

\subsection{Wie wirkt sich Partialisierung auf den Bezug zum alltäglichen Deutschunterricht aus?}

Wer grundsätzlich oder vornehmlich quantitativ empirisch forscht, privilegiert aufgrund bestimmter Erhebungsmethoden ganz bestimmte fachliche Gegenstände, die gut zerlegbar sind, wie Orthografie - immer wieder die Silbe in vielerlei Gestalt -, Schriftspracherwerb in der Primarstufe, überhaupt den Anfangsunterricht oder literarische Kleinformen, die ein Minimum an fachbezogenem Kontextwissen voraussetzen.

Viele zentrale Gegenstände des Deutschunterrichts liegen außerhalb der Reichweite quantitativer Verfahren und werden deshalb auch nicht beforscht. Die enge thematische Begrenzung quantitativer empirischer Studien gilt es also ebenso wahrzunehmen wie den damit verbundenen Gewinn an Exaktheit. Konsequenz der methodeninduzierten Begrenzung sind mitunter bereits Fragestellungen, die so wenig mit Praxis und Theorie des Deutschunterrichts zu tun haben, dass sie 
lediglich für die ForscherInnen interessant sind. Dass bei quantitativer empirischer Fachunterrichtsforschung eine Vielzahl von Variablen(klassen) wirksam und verallgemeinerbare Resultate schwer zu gewinnen sind, darauf hat Reinold Funke (2014) nachdrücklich hingewiesen.

Als Fazit „eine[r] metaanalytische[n] Bestandsaufnahme“ aller von ihm "aufgefundenen Studien zu Lesen durch Schreiben" formuliert er, dass die Aussagekraft der vorliegenden empirischen Studien „[...] durch methodologische Beschränkungen begrenzt wird“. Funke hebt drei Aspekte hervor: (1) dass „die Lernergebnisse [...] nicht nur auf die „Erstunterrichtsmethode“, sondern auch auf „Merkmale der Lehrkräfte“ zurückzuführen sind; (2) dass „eine eingeschränkte Validität der Ermittlung der Lernergebnisse [...] das Zustandekommen von Zufallsbefunden befördert haben“ könnte; (3) dass keine der vorliegenden Studien das Lesen durch Schreiben-Konzept "systematisch an seinem eigenen Anspruch“ messe, nämlich dass die nach dieser Methode unterrichteten Kinder „ein positiveres Verhältnis zur Nutzung der Schriftsprache gewinnen als andere" (Funke 2014, S. 37).

Was quantitativ empirisch funktioniert, ist die Erfassung von Aspekten orthographischer Kompetenz (Fay 2010; Voss et al. 2007), die Erfassung der Fähigkeit zur Produktion stark formalisierter schriftlicher Texte (Neumann 2012; 2014) und die Erhebung des Leseverstehens in Bezug auf pragmatische Alltagstexte (Bremerich-Vos/Böhme 2009).

Fazit: Letztlich zeigen sich hier ganz ähnliche Effekte wie bei der Lehr-/Lernforschung allgemein: Sehr präzise Ergebnisse bleiben ohne Relevanz für den alltäglichen Deutschunterricht. Denn die Ergebnisse der empirischen Beforschung fördern sehr Unterschiedliches zutage, sodass die zentrale Frage, wie die unterschiedlichen Ergebnisse auf den Deutschunterricht und aufeinander bezogen werden, als Problem bestehen bleibt.

\subsection{Wie wirkt sich Partialisierung auf die systematische Verortung der Deutschdidaktik aus?}

Ob DeutschdidaktikerInnen als SozialwissenschaftlerInnen oder als VertreterInnen einer germanistischen Disziplin forschen, macht auch im empirischen Zusammenhang einen Unterschied. Es geht in der Tat um unterschiedliche Interessenlagen. Wenn DeutschdidaktikerInnen als SozialwissenschaftlerInnen forschen, dann haben sie natürlich neben dem SchülerInnen- und LehrerInnenverhalten und der unterrichtlichen Interaktion auch die fachlichen Gegenstände im Blick. Aber sie betrachten diese Variablen als gleichrangige Stellschrauben. Wenn DeutschdidaktikerInnen als Literatur- oder SprachwissenschaftlerInnen 
forschen, dann haben sie auch das SchülerInnen- und LehrerInnenverhalten und die Interaktion im Blick, aber eben als Bedingungen, unter denen fachliche Gegenstände optimal rezipierbar oder produzierbar werden ${ }^{4}$. Etwa so, wie BiologInnen Zellforschung mit anderen Interessen betreiben als MedizinerInnen.

Eine Deutschdidaktik, die sich den fachlichen Gegenständen und damit der Germanistik verpflichtet fühlt, stellt bezogen auf die fachlichen Gegenstände und fachbezogenen Kompetenzen andere Fragen als eine Deutschdidaktik, die sich als Sozialwissenschaft versteht. Aus sozialwissenschaftlicher Perspektive stellt sich beispielsweise die Frage, ob die Lehrperson stoff- oder kompetenzorientiert unterrichtet, während aus germanistischer Perspektive interessiert, ob ein bestimmter Gegenstand zum Erwerb einer bestimmten Kompetenz geeignet ist, ob er prototypischen Charakter hat, ob der fachliche Gegenstand als solcher gewürdigt wird und ggf. warum das nicht der Fall ist. Auch LernforscherInnen wie Elsbeth Stern machen sich im Anschluss an Shulman für ein pedagogical content knowledge - für das fachspezifische pädagogische Wissen - stark und sehen die Aufgabe der Fachdidaktik in der Erzeugung fachspezifischen pädagogischen Wissens (Felten/Stern 2012, S. 12). Sie betrachten es aber als Systemstelle in einer pädagogisch-psychologisch orientierten Unterrichtswissenschaft.

Fazit: In Bezug auf die systematische Verortung der Deutschdidaktik erzeugt die Partialisierung große Vielfalt und Auffächerung, was für die Außenperspektive verwirrend erscheinen mag und einer stabilen Position der Deutschdidaktik im akademischen Kontext wenig förderlich sein dürfte. Deutlich wird allerdings, dass Deutschdidaktik VertreterInnen ganz unterschiedlicher Herkunft rekrutiert. Neben GrammatikerInnen und TextlinguistInnen stehen LiteraturhistorikerInnen und LiteraturtheoretikerInnen, neben Strukturalisten stehen Hermeneutiker, neben SchreibforscherInnen stehen Lehr-/ LernforscherInnen mit mehr oder weniger ausgeprägten statistischen Neigungen und neben Theaterpädagogen Kinder- und JugendliteraturforscherInnen.

\subsection{Optionen für die Forschung}

Partialisierung findet auf inhaltlicher und methodologischer Ebene statt. Während die inhaltliche Partialisierung sowohl den Deutschunterricht als auch die Deutschdidaktik als wissenschaftliche Disziplin betrifft, ist die abgegrenzte Viel-

4 Unterstützung und Inspiration erhält diese Überlegung durch den von Christoph Bräuer entwickelten Ansatz der Unterrichtsrahmenanalyse, die - in Abgrenzung zu anderen kommunikationstheoretischen Ansätzen - die gegenstandsbezogene Rahmung zentral setzt. (vgl. Bräuer 2011). 
falt an Forschungsmethoden Sache der wissenschaftlichen Disziplin. Hier geht es zum einen um die Separation von hermeneutischen und empirischen Verfahren und zum anderen um deren jeweilige Untergliederungen.

Auf einer zweiten Ebene ist im Rahmen quantitativer Empirie insofern von Partialisierung zu sprechen, als bestimmte Verfahren sowohl die fachlichen Gegenstände als auch die Aktivitäten der Probanden so stark zerlegen müssen, dass die Ergebnisse letztlich der reinen Grundlagenforschung zuzuschreiben sind, die zwar DFG-fähig, aber mit dem Verlust von Alltags- und Praxisrelevanz verbunden ist. ${ }^{5}$ Der Bezug zum "faktisch vorkommenden Deutschunterricht“ (Bremerich-Vos 2014, S. 11) wird Sache der Anwendungs- und Interventionsforschung. Letzterer sind z. B. wichtige Lesestrategiestudien deutschdidaktischer (Rosebrock et al. 2010) und psychologischer Provenienz (McElvany 2008; Müller 2014) zu verdanken.

Bei der Aufspaltung in Grundlagen- und Anwendungsforschung handelt es sich nicht nur um eine Trennung von Erkenntnisgenerierung und praktischer Problemlösung, sondern auch um ein Renommeegefälle. Das zeigt sich nicht zuletzt in der Förderpraxis der DFG. Mit diesen inhaltlichen, v. a. aber methodischen Partialisierungen verbinden sich unterschiedliche systematische bzw. disziplinäre Verortungen. Nach wie vor erscheinen Germanistik und Sozialwissenschaften als naheliegende Optionen. Dabei fällt ins Gewicht, dass Deutschdidaktik als empirisch forschende sozialwissenschaftliche Disziplin größere Chancen auf wissenschaftliche Anerkennung hat als Deutschdidaktik als Teildisziplin der germanistischen Literatur- bzw. Sprachwissenschaft. ${ }^{6}$ Auf dieser Folie bedeutet Partialisierung nicht nur sortierte Vielfalt, sondern auch Competition und Selektion mit dem Ziel „survival of the fittest“.

Da sich die Vielfalt der Bereiche, mit denen es Deutschdidaktik zu tun hat, nur schwer unter das Dach eines eigenen spezifischen Forschungsprofils bringen lässt, ist Partialisierung in einem gewissen Rahmen geboten. Zugleich aber gilt: Partialisierung ist eine Form der Ausdifferenzierung und Spezialisierung, die zur Bildung esoterischer Zirkel im Fleckschen Sinne führt. Das bedeutet in der Konsequenz die Bildung von Stämmen und Unterstämmen. Als zentrales Problem erweist sich jedoch, dass wir als DeutschdidaktikerInnen zu wenige sind, um zahlreiche Stämme und Unterstämme bilden zu können. Damit geht als weiteres Problem einher, dass es zu wenig Kommunikation zwischen den „Stämmen“ gibt,

5 Allerdings erzielen auch ForscherInnen, die theoriegeleitet analysieren und reflektieren, keineswegs immer Ergebnisse, die für den professionellen Alltag bedeutsam sind.

6 Daran dürften auch die germanistischen Disziplinen einen gewissen Anteil haben. 
vor allem in Form kritischer Auseinandersetzung miteinander. Das hat wiederum damit zu tun, dass das Gespräch unter den ForscherInnen bei der hochgradigen Spezialisierung weniger fachlich als methodologisch verläuft und zudem immer schwieriger wird.

\section{Eklektizismus}

Demgegenüber zielt Eklektizismus darauf, Deutschdidaktik als eine angewandte Wissenschaft unter ein großes Dach zu bringen. Da die Germanistik als traditionelle Orientierung in weiten Teilen zur Kulturwissenschaft geworden ist und die Didaktik ihrerseits auch Affinität zur Pädagogik zeigt, haben sich sowohl die Kulturwissenschaften als auch die Pädagogische Psychologie bzw. empirische Lehr-/ Lernforschung als attraktive, überwölbende Domänen herauskristallisiert. Schaut man von außen auf die Disziplin, so erscheint die Situation der Deutschdidaktik wie die von Buridans Esel: Links der Heuhaufen Germanistik/Kulturwissenschaft, rechts der Heuhaufen Lehr- und Lernforschung und in der Mitten verhungert der Esel Fachdidaktik, der sich nicht entscheiden kann. ${ }^{7}$

\subsection{Welche Merkmale kennzeichnen eklektizistische Positionen?}

Kennzeichen eklektizistischer Konzeptionen sind alle Kombinationen mit Synoder Sym-, mit „inter-“ oder „trans-“ sowie Kombinationen aus syndetischen und asyndetischen Reihungen. Diese Verbünde verweisen auf eine deutschdidaktische Position, die die Breite sucht und der Vielfalt mit Integration resp. Inklusion begegnet, nicht zuletzt sowohl um der Einheit des Faches willen als auch im Hinblick auf die Integration neuer Herausforderungen. Aktuell erscheint transdisziplinäre Ausrichtung sowohl in Bezug auf die Gegenstände als auch auf methodische Verfahren als deutlichster Indikator. Eklektizistisch ist dieser Vorgang, weil er sich nur einzelner Versatzstücke bedient und folglich etwas Beliebiges an sich hat, sodass das Ergebnis auch immer wieder anders ausfallen könnte.

Streng definiert unterscheidet sich Eklektizismus von Pluralismus dadurch, dass er „willkürlich Ansätze und Fragmente“ von Forschungsbereichen und -methoden vermischt (Miller 2012, S. 6). Folglich sollten wir uns darüber verständigen, wo die Grenze zwischen ,geringerer Stilreinheit' und ,Eklektizismus' verläuft, zumal nicht alle diese Ansätze immer gleich „willkürlich“ sind. Es gibt durchaus

7 Diesen einprägsamen Vergleich habe ich aus Jakob Ossners Kommentierung dieses Beitrags übernommen. 
Gründe für ein relationales Vorgehen, wenn ein Problem mit komplementären Ansätzen in den Blick genommen wird, und eine nachvollziehbar begründete Methodentriangulation wird man auch nicht „willkürlich“ nennen wollen.

\subsection{Wie wirkt sich Eklektizismus auf die systematische Verortung der Deutschdidaktik aus?}

Als paradigmatisch für eklektische Positionen können vor allem zwei Konzepte gelten: Kepsers Plädoyer für „Deutschdidaktik als eingreifende Kulturwissenschaft“ (Kepser 2013) und Frederkings Entwurf einer „Deutschdidaktik als transdisziplinäre, anwendungs- und grundlagenorientierte empirische Wissenschaft ${ }^{\text {" }}$ (Frederking 2014, S. 109). Wenn Kepser die Deutschdidaktik kulturwissenschaftlich verortet, dann schließt er „all ihre [...] Teildisziplinen“ ein (Kepser 2013, S. 54) und geht davon aus, dass die Deutschdidaktik sich immer schon "Gegenständen zugewandt [habe], die in den Bezugswissenschaften und hier allen voran der Germanistik vernachlässigt worden sind“ (ebd., S. 57). Letztlich sind es „Inter- und Transdisziplinarität sowie [...] Methodenpluralismus“ (ebd., S. 58), die Kepsers Position als eklektizistisch kennzeichnen. Wieser fragt allerdings zu Recht nach dem Orientierungswert einer Verortung in den Kulturwissenschaften, wenn diese, wie Kepser selbst feststelle, „keinem einheitlichen Paradigma folgen, weder im Hinblick auf ihre Gegenstände noch im Hinblick auf ihre Methodologie“ (Wieser 2015, S. 19). Die Antwort auf Wiesers Frage besteht aber gerade in der Attraktivität des Eklektizismus. Insofern ist es nur konsequent, wenn Wieser in Kepsers Option auch einen „Befriedungsversuch“ sieht, der es erlaube, die heterogenen Tendenzen der Deutschdidaktik unter ein großes Dach zu bringen. Der Preis einer solchen Befriedung bliebe allerdings eine massive Unschärfe der disziplinären Verortung.

Anders stellt sich der transdisziplinäre Charakter der Deutschdidaktik bei Frederking dar. Auf der Grundlage der Transdisziplinarität ziele

deutschdidaktische Forschung auf die konzeptionelle Entwicklung, theoretische Fundierung und empirische Erforschung fachspezifischer Lehr-Lern-Prozesse und ihrer inhaltlichen und methodischen Grundlagen innerhalb und außerhalb der Schule" und ha[be] „mithin fachliche Lerninhalte und fachspezifische Lehr-Lern-Prozesse zum Gegenstand, die sich zwischen Lehrenden und Lernenden vollziehen und bei denen zwischen fachspezifischen theoretischen Konzepten und praktischen Umsetzungen im Fachunterricht oder in anderen Anwendungskontexten zu unterscheiden [sei] (Frederking 2014, S. 111 f.).

Wissenschaftliche Satisfaktionsfähigkeit der Deutschdidaktik wird in Frederkings Perspektive [jedoch] „durch wissenschaftliche Exzellenz“ gewährleistet. Denn „eine ausgewiesene Drittmittelstärke der Deutschdidaktik w[erde] in 
Zeiten einer ökonomisierten Deutschdidaktik als Qualitätskriterium gewertet - ob uns dies nun gef[alle] oder nicht" (Frederking 2014, S. 113 f.). Dies gehe allerdings nur ,über Forschungsprojekte auf der Ebene von DFG, BMBF, Schweizer Nationalfond, EU etc.“ (ebd.). Eklektisch ist diese Position insofern, als Frederking auf die Aufhebung der ,inneren Distanz gerade der Literaturdidaktik gegenüber den Methoden und Erkenntnisweisen der exakten Natur-, Sozial- und Bildungswissenschaften“ (ebd., S. 117) zielt und damit auf deren Integration in die Deutschdidaktik. Hier grenzt er sich in aller Deutlichkeit von Abraham ab, gegen dessen - ebenfalls eklektizistisch zu nennendes - „Ganzheitspostulat" er den Anspruch auf „exakte [...] empirische [...] Forschung“ erhebt, die „im Bereich des sprachlich-literarischen Lernens ,Einzelkomponenten ' - ob testbar oder nicht - ,unabhängig voneinander' [...] beschreib [e] “ (ebd.). Dass Forschung auf separierende Werkzeuge angewiesen ist, wird auch Abraham nicht bestreiten. Strittig ist wohl eher der Zuschnitt der Einzelkomponenten bzw. deren Zerlegungsgrad. Auf diesen partialisierenden Aspekt geht Frederking gar nicht ein. Einzelkomponenten sind auch unter Berücksichtigung ihres "Zerlegungsfaktors" zu diskutieren. Frederking unterstellt jedoch, dass wir - „grosso modo gesprochen - scheinbar gar nicht genau wissen wollen, ob unsere literaturdidaktischen Annahmen einer kritischen wissenschaftlichen Überprüfung standhalten - erst recht nicht, wenn diese mit den härtesten wissenschaftlichen Gütekriterien vorgenommen werden: mit denen der quantitativen Forschung“ (ebd., S. 116).

Ebenso wie Kulturwissenschaft als disziplinärer Status ist auch die Orientierung an „den Methoden und Erkenntnisweisen der exakten Natur-, Sozial- und Bildungswissenschaften“ (ebd., S. 117) vor allem deshalb attraktiv, weil - holzschnittartig gesprochen - der Fokus vom „Unterricht“ auf „Wissenschaft/Forschung" verlagert wird, sodass, wie Wieser (2015, S. 19) formuliert, „die Suche nach geeigneten Vermittlungsgegenständen [sowie] die Ausbildung von Lehrerinnen und Lehrern" nachgelagert erscheint.

\subsection{Wo liegt das Problem des Eklektizismus?}

Blickt man auf die Einheit des Fachs, dann bedeutet die Frederking-AbrahamKontroverse einen Riss. Das ist insofern nicht verwunderlich, als sich der Eklektizismus unterschiedlicher Elemente bedient, die er unter einer Überschrift versammelt. Zum anderen fehlt in der Breite und Fülle der Disziplin der Umriss eines Zentrums. Anders formuliert: Während WissenschaftlerInnen, die in der Deutschdidaktik nach Partialisierung streben, ein Denkkollektiv ausbilden wollen und einen Denkstil ausbilden, bilden eklektische Wissen- 
schaftlerinnen kein kritisches Kollektiv und entwickeln folglich auch keinen Denkstil. Allerdings wird man jedem einzelnen wohl einen Denkstil nicht ganz absprechen können.

\subsection{Wie wirkt sich Eklektizismus auf den Bezug zum alltäglichen Deutschunterricht aus?}

Fragt man nach dem Bezug eklektizistischer Konzepte zum alltäglichen Deutschunterricht, dann sind die Antworten zwiespältig. Einerseits ist die stete Erweiterung der Domäne wenig geeignet, um Fragen des Unterrichtsalltags zu fokussieren. Andererseits zielt Frederkings Definition der Deutschdidaktik auf „fachliche Lerninhalte und fachspezifische Lehr-Lern-Prozesse“ und schließt deren „konzeptionelle Entwicklung“ (Frederking 2014, S. 111) ausdrücklich ein, sodass Entwicklung und Implementation durchaus Optionen sind - aber auch nicht mehr. Auch Kepsers Konzept sieht Eingriffe als ein „Generieren von Veränderungs- und Verbesserungsvorschlägen“ (Kepser 2013, S. 59) vor, was mehr bedeutet als Rekonstruktion. Kepsers Konzept setzt auch insofern auf Eklektizismus, als er „über bildungs- und erziehungswissenschaftlich konturierte Vorhaben hinaus" auch „Potenzial für neue Forschungskooperationen“ sieht (ebd., S. 61). Bei aller Unterschiedlichkeit der Ansätze dürfte Frederking Kepser darin zustimmen, dass „Deutschdidaktik [...] in erster Linie Wissenschaft“ ist und sich „als solche [...] zuallererst der Forschung verpflichtet zu fühlen" hat (ebd., S. 60).

\subsection{Fazit}

Sowohl Partialisierung als auch Eklektizismus lassen sich als Reaktionen auf die Breite und Vielfalt der Domäne „Deutschdidaktik“ beschreiben. Partialisierung setzt auf Separierung und Denkstil, Eklektizismus auf die Gesamtheit des Fachs und auf Vernetzung mit anderen Disziplinen. Eklektizismus repräsentiert in gewisser Weise die Konzeption des Schulfachs, Partialisierung den Denkzwang wissenschaftlicher Denkkollektive und vornehmlich die methodisch gebotenen Reduktionen und Beschränkungen. Sowohl partialisierende als auch eklektizistische Ansätze verfolgen sinnvolle Ziele, die aber einander weitgehend ausschließen. Insofern stellen sie ein Dilemma dar. Für die Disziplin wird das zum Problem, weil zwischen den beiden polarisierenden Bestrebungen ein blinder Fleck entstanden ist, der die konkreten Inhalte und Herausforderungen des Deutschunterrichts neutralisiert. 


\section{Auswege aus dem Dilemma?}

Was also tun, um der Forderung nach Relevanz der Disziplin für die unterrichtliche Praxis gerecht zu werden, ohne den wissenschaftlichen Ansatz aufzugeben?

\subsection{Mut zur Professionswissenschaft}

Eine Option bestünde darin, zu akzeptieren, dass Deutschdidaktik faktisch eine Professionswissenschaft ${ }^{8}$ ist. Wie auch z. B. die Rechtswissenschaft ist Deutschdidaktik eine Disziplin, die für ein staatlich geregeltes Professionsfeld ausbildet. Darüber hinaus besteht eine enge Kopplung der Forschungsfragen an die Praxis des Deutschunterrichts. Und das breite Spektrum an Medien und Formaten, mit denen Forschungsergebnisse kommuniziert werden, hat nicht zuletzt damit zu tun, dass Publikationen sich nicht nur an die wissenschaftliche Community richten, sondern auch auf die Unterrichtspraxis bezogen sind.

Als Wissenschaft, die Praxis nicht nur erforscht, sondern auch ,auf Praxis zielt“ (Ossner 1999, S. 37), ist sie „zukunftsgerichtet“ (ebd.). Sie verknüpft Rekonstruktion mit Konstruktion, Deskription mit Normfragen. Folglich hat sie eklektische Züge. So plädiert Ossner (2001, S. 23) (mit Hartmut Günther) für eine Deutschdidaktik, die „mit empirischen Verfahren die vorfindliche Realität [beschreibt] und [...] mit didaktischen Modellen auf ihre Gestaltung [zielt]“ (Ossner 2002, S. 23). Auch wenn man berücksichtigt, dass „vorfindliche Realität“ empirisch schwieriger zu erfassen ist, als wir das vor 15 Jahren angenommen haben, so spricht doch viel für eine solche Bestimmung wissenschaftlicher Deutschdidaktik. Es geht vor allem, aber nicht nur darum, dass sie Entscheidungshilfen bietet und Entscheidungsmöglichkeiten diskutiert, dass sie gesellschaftliche Verantwortung trägt für das, was im Deutschunterricht gelernt werden kann. Es geht auch um die Frage, wer es tut, wenn es die Deutschdidaktik nicht tut. Vieles weist darauf hin, dass die Bildungsadministration dafür eher die Unterstützung durch die empirische Bildungsforschung sucht, weil deren Transferpotenzial größer ist, und eben nicht durch die wissenschaftliche Deutschdidaktik. Ins Gewicht fällt hier auch, dass die empirische Bildungsforschung zwar Zahlen liefert, mit denen die Politik gut umgehen kann, aber die Komplexität dessen, was in der Schule passiert, durch diese Maße unzulässig verengt wird.

8 Gegenüber den „Professionsfakultäten“ der Rechtswissenschaft, der Theologie und der Medizin hat sie allerdings das Problem, weder eine Fakultät zu sein noch eigene Studiengänge zu bieten. Vgl. auch die Analysen und Empfehlungen des Wissenschaftsrats (2012) zur Rechtswissenschaft. 
Die fachlichen Aspekte werden dann durch die Bildungsmedien, sprich Deutschbücher, geliefert. Indem Ossner von der Deutschdidaktik Zielvorgaben und Handlungsalternativen als Entscheidungshilfe für LehrerInnen erwartet, hält er fest am germanistischen Kern der Disziplin.

Es mag einen kleinen Exkurs wert sein, nach den Gründen zu fragen, die die Community daran hindern, sich beherzt als Professionswissenschaft zu verorten und wissenschaftlich an Entscheidungshilfen in Bezug auf Ziele und Handlungsweisen $\mathrm{zu}$ arbeiten.

Nachvollziehbar und berechtigt ist die Furcht vor wissenschaftlicher Insuffizienz und vor Unterlegenheit gegenüber anderen Disziplinen, die - wie Bromme und Brüggemann (2006) urteilen - weniger Probleme haben, einen „Denkstil“ zu entwickeln. Auch sind sowohl „die Freiheitsgrade“ als auch „die Möglichkeit zu Stilreinheit und Kohärenz der Theoriebildung" in der reinen Grundlagenforschung „deutlich höher“ (Bromme/Brüggemann 2006, S. 7) als in der anwendungsorientierten Grundlagenforschung bzw. in den Professionswissenschaften.

Zudem ist es mühsam, sich mit Bildungspolitik, Bildungsadministration, den Bildungsmedien und nicht zuletzt den Bildungsforschern auseinanderzusetzen und ggf. auch die Erfahrung ausbleibenden Erfolgs zu machen.

Sofern FachdidaktikerInnen - stärker als VertreterInnen anderer Wissenschaften mit „enge[r] Theorie-Praxis-Verklammerung“(WR 2012) - sich als WissenschaftlerInnen nicht primär für Ziele und Handlungsweisen im Professionsfeld interessieren, könnte das - spekulativ gesprochen - auch mit der Langlebigkeit einiger der „Tabus“ zu tun haben, die Adorno 1965 „über dem Lehrberuf“ vermutet hat. Adorno selbst betont den spekulativen Charakter seiner Erklärung der Tatsache, dass

gerade [die] Begabtesten unter den akademischen Absolventen, die das Staatsexamen gemacht haben, [...] starken Widerwillen [äußern] gegen das, wozu dies Examen sie qualifiziert, und was man nach diesem Examen eigentlich von ihnen erwartet (Adorno 1965, S. 656).

Den von ihm gebrauchten Tabubegriff definiert Adorno als

einigermaßen streng, im Sinne des kollektiven Niederschlags von Vorstellungen, die [...] ihre reale Basis in weitem Maß verloren haben, [...], die sich aber, als psychologische und soziale Vorurteile, zäh erhalten und ihrerseits wieder in die Realität zurückwirken, reale Kräfte werden (ebd., S. 657).

Adorno unterstellt, dass „der Lehrberuf, verglichen mit anderen akademischen Berufen wie dem des Juristen oder des Mediziners, ein gewisses Aroma des gesellschaftlich nicht ganz Vollgenommenen [besitze]“ (ebd., S. 657). Während Juristen und Ärzte ihren Beruf auch oder überwiegend freiberuflich ausübten und 
„solcher Unbeengtheit wegen höher eingeschätzt“ würden, seien Lehrer „in eine Beamtenhierarchie vermauert und gesichert" (ebd., S. 660). Adorno unterstellt einen „soziale[n] Gegensatz“, einen

Bruch in der bürgerlichen Schicht selber, wenigstens im Kleinbürgertum, zwischen den Freien, die mehr verdienen, deren Einkommen aber nicht garantiert ist, und die eines gewissen Airs von Kühnheit, von Ritterlichkeit sich erfreuen mögen, und andererseits den pensionsberechtigten Festangestellten und Beamten, die man zwar wegen ihrer Sekurität beneidet, jedoch als Amts- und Bürohengste, mit festen Arbeitszeiten und Leben nach der Ochsentour, über die Achsel ansieht (ebd., S. 660 f.).

Als wirksamer Rest erweist sich sowohl die Begrenztheit der Karrierechancen im Lehrberuf als auch die Tatsache, dass leistungsstarke Abiturienten sich signifikant weniger für ein Lehramtsstudium interessieren. ${ }^{9}$

Einen weiteren Aspekt der „Mißachtung des Lehrers“ sieht Adorno darin,

daß man ihn, weil er in eine Kinderwelt eingespannt ist, die entweder ohnehin die seine ist oder der er sich anpaßt, nicht ganz als Erwachsenen betrachtet, während er ein Erwachsener ist und seine Ansprüche aus seinem Erwachsensein ableitet (ebd., S. 665).

Wohlgemerkt: Adorno spricht von „Tabus über dem Lehrberuf“, „nicht von der Wirklichkeit des Lehrberufs, auch nicht von der wirklichen Verfassung der Lehrer“, aber er sieht die Tabus auch "nicht ganz unabhängig“ von der Wirklichkeit (ebd., S. 672).

Dennoch ist der alltägliche Deutschunterricht das Feld, auf dem wir uns auskennen (sollten) und auf dem wir etwas zu bieten haben, das weder die Bildungsadministration noch die Bildungsmedien leisten können. Auch nicht die BildungsforscherInnen, die den fachlichen Gegenständen indifferent gegenüber stehen. Diese Institutionen konkurrieren mit uns um dieses Feld.

Impulse, sich deutschdidaktisch auf diesem Feld zu engagieren, geben zwei zukunftsweisende und erfolgversprechende Forschungsrichtungen, die ihrerseits durchaus eklektische Züge tragen. Das gilt sowohl für die anwendungsorientierte Grundlagenforschung als auch für die entwicklungsorientierte Bildungsforschung, die im Folgenden kurz kommentiert werden sollen.

\subsection{Anwendungsorientierte Grundlagenforschung}

Dass in Wissenschaften mit enger Kopplung von Wissenschaft und Praxis wie der Rechtswissenschaft, den Wirtschaftswissenschaften, wie der Medizin und der

9 http://www.hochschulbildungsreport2020.de/handlungsfeld/lehrer-bildung/ fokus/2014.html. Abgerufen am 19.01.2017. 
Psychologie nicht nur Grundlagenforschung gefördert werden kann, hat auch die DFG bewogen, „,anwendungsorientierte Grundlagenforschung“ als neue Förderlinie zu etablieren.

In Bezug auf die Psychologie haben Brüggemann und Bromme (2006) zu profilieren versucht, worin die Schwierigkeit anwendungsorientierter Grundlagenforschung im Vergleich mit reiner Grundlagenforschung besteht. Was die beiden Autoren für die anwendungsorientierte Grundlagenforschung im Bereich der Psychologie konstatieren, sollte sich auf die Deutschdidaktik übertragen lassen.

Anwendungsorientierte Grundlagenforschung ist [...] Grundlagenforschung im Sinne von Generalisierbarkeit und theoretischer Anbindung. Sie ist aber keine ,reine' Grundlagenforschung, da sie aus praktischen Problemen entsteht, was gleichzeitig die Perspektive der potenziellen Problemlösung einschließt. D. h. ,anwendungsorientierte Grundlagenforschung intendiert dezidiert praktische Effekte bzw. praktischen Nutzen und zieht sie auch zur Begründung und Beschreibung ihrer Fragestellungen heran. Auch ,reine 'Grundlagenforschung kann natürlich praktische Effekte und Nutzen haben, diese sind jedoch nicht Teil des Begründungszusammenhangs bei der Entwicklung der Fragestellung. (Brüggemann/Bromme 2006, S. 3)

Untersuche man Lernen ,aus rein grundlagenpsychologischer Sicht“, könne man sich dabei empirisch „,auf die Phänomene (Verhaltensweisen, Gehirnströme, Augenbewegungen etc.), die als empirischer Referenzpunkt aus der Perspektive der jeweiligen Theorie in Frage kommen", konzentrieren (ebd., S. 7). Andere Einflüsse und Variablen, wie z. B. „Intentionen, motivationale Zustände, Unterschiede von Lerninhalten“, spiel[t]en nur unter bestimmten peripheren Bedingungen eine Rolle, so dass es grundsätzlich möglich ist, „eine bestimmte Theorie stilrein zu formulieren“ (ebd., S. 8). Anders verhalte es sich in der anwendungsorientierten Grundlagenforschung:

Wenn - ausgehend von den praktischen Möglichkeiten des Lehrens und Lernens mit Computern - der Frage nachgegangen werden soll, unter welchen Bedingungen Text und Bild beim Lernen am PC sich gut ergänzen, wann sie sich stören und welche Lerner von welchen Varianten profitieren, dann sind für die Analyse derartiger Lernprozesse Variablenklassen zu berücksichtigen, die nicht mehr allein in einem theoretischen Rahmen zu formulieren sind. In diesem Sinne ist hier von Heterogenität und geringerer Stilreinheit die Rede, d.h. es müssen Elemente unterschiedlicher Theorien und damit auch Konstrukte aus unterschiedlichen innerpsychologischen Diskursen herangezogen werden, um dem jeweiligen Problem gerecht zu werden. (ebd.; Herv. J.K.)

Ein Desiderat wären beispielhafte Fragestellungen für anwendungsorientierte Grundlagenforschung in der Deutschdidaktik. Gibt es bereits Beispiele? Welche 
praktischen Probleme erscheinen so drängend, dass die Disziplin auf sie reagieren sollte, um Lösungen zu entwickeln?

\subsection{Unterrichtsforschung als Entwicklungsforschung}

Weiter gehend ist Gabi Reinmanns Vorschlag, Bildungsforschung auch als Entwicklungsforschung zu konzipieren. Aus der Perspektive der Pädagogischen Psychologie plädiert sie (2010, S. 250) zum einen dafür, in einem erkenntnistheoretischen Diskurs zu klären, „mit welchen Prämissen und Werkzeugen wir antreten“. Sowohl in der „Gegenüberstellung von Grundlagenforschung und Anwendungsforschung" als auch „von Theorie und Empirie“ vermutet sie "falsche Dichotomien“, die dazu beitragen,

dass eine Ko-Existenz, vergleichbare Wertschätzung und sinnvolle Verknüpfung beschreibender, erklärender, begründender und entwickelnder Forschungstätigkeiten in den Bildungswissenschaften so schwierig sind und in der Forschungsförderung mitunter auf großes Unverständnis stoßen (ebd).

Zudem seien „empirische wie auch hermeneutische Verfahren letztlich der Vergangenheit zugewandt“, sodass es „empirischen wie hermeneutischen und kombinierten Verfahren nach wie vor an einer deutlichen und eigenen Perspektive auf Gestaltung der Zukunft“ fehle (Reimann/Sesink 2011, S. 4 f.). „Bildung [...] und damit auch die Bildungswissenschaft [seien jedoch durch] ihre Zugewandtheit zur Zukunft" (vgl. auch Ossner 1999, S. 37; Reimann/Sesink 2011, S. 18) ausgezeichnet. Um eben diese Zukunftsorientierung ,in den Forschungsprozess herein zu holen“, erscheint ihr „die schon oft postulierte wechselseitige Ergänzung und Stützung von Empirie und Hermeneutik" nicht hinreichend, da beide gleichermaßen Mangel an Zukunftsbezug haben. Deshalb plädiert sie zum anderen dafür, „dass es neben der Erforschung des ,Ist-Zustands' auch einen ,Soll-Zustand' und einen ,Weg vom Ist zum Soll' gibt, den man wissenschaftlich betrachten, begleiten oder initiieren kann" (Reinmann 2010, S. 251). Hier warnt Ossner vor dem naturalistischen Fehlschluss, dass aus Beschreibungen Leitlinien für ein Handeln abzuleiten wären. Während Reinmann und Sesink (2011, S. 18) „es als wissenschaftliche Pflicht “ ansehen, „entsprechend der im Interesse stehenden Forschungsziele und -fragen einen angemessenen wissenschaftlichen Weg zu gestalten, um diese Ziele zu erreichen bzw. um diese Fragen zu beantworten“ (Reinmann 2010, S. 248), unterscheidet Ossner zwischen Angebot und Nutzung. Er ist der Überzeugung, dass der Dienst der Fachdidaktik darin besteht, Probleme im Handlungsfeld verstehbar zu machen. Das bedeutet: Instrumente bereitzustellen, aber nicht zu sagen, wie mit dem Instrument umzugehen ist. Wissenschaft könne immer nur die Bedingungen nennen, unter denen Ziele erreichbar werden. 
Abschließend bleibt zu konstatieren, dass die "Zugewandtheit zur Zukunft" keine einfache Angelegenheit ist. Denn Zukunftskonzepte hängen immer von der jeweiligen Gegenwart ab. Die hier verhandelte ist facettenreich und unübersichtlich. Wenn wir, wie Dorothee Wieser (in diesem Band) mit Hubert Ivo vorschlägt, "eine offene und durchaus auch pragmatische Diskussion über die Funktionen der Deutschdidaktik [führen wollen] (vgl. Ivo 1977, S. 138)“, dann gälte es auch, den „blinden Fleck“ in allen Fragerichtungen aufzuspüren, auch in der hier vertretenen.

\section{Literatur}

Adorno, Theodor W. (2003) [1965]: Tabus über dem Lehrberuf. In: Adorno, Theodor W.: Gesammelte Schriften in zwanzig Bänden. Kulturkritik und Gesellschaft I. Prismen ohne Leitbild. Frankfurt /M.: Suhrkamp, S. 656-673.

Bildungsstandards im Fach Deutsch für den Mittleren Schulabschluss. Beschluss der Kultusministerkonferenz vom 4.12.2003. München: Luchterhand.

Bildungsstandards im Fach Deutsch für den Primarbereich (Jahrgangsstufe 4). Beschluss der Kultusministerkonferenz vom 15.10.2004. München: Luchterhand.

Bildungsstandards im Fach Deutsch für die Allgemeine Hochschulreife (Beschluss der KMK vom 18.10.2012). http://www.kmk.org/dokumentation/ veroeffentlichungen-beschluesse/bildung-schule/qualitaetssicherung-inschulen.html\#c7035. Abgerufen am 13.03.2015.

Bräuer, Christoph (2011): Die Unterrichtsrahmenanalyse - ein Beobachtungsinstrument für die praktische Forschung wie die forschende Praxis. In: Kommunikation und Interaktion im Unterricht. OBST. Jg. 2011. H. 80, S. 13-30.

Bremerich-Vos, Albert (2013): Standards und Kompetenzdiagnostik im Fach Deutsch - 12 Jahre nach PISA. In: Fay, Johanna (Hrsg.): (Schrift-) Diagnostik heute. Baltmannsweiler: Schneider, S. 5-24.

Bremerich-Vos, Albert et al. (2012): Kompetenzstufenmodelle im Fach Deutsch. In: Stanat, Petra et al. (Hrsg): Kompetenzen von Schülerinnen und Schülern am Ende der vierten Jahrgangsstufe in den Fächern Deutsch und Mathematik. Ergebnisse des IQB-Ländervergleichs 2011. Münster u. a.: Waxmann, S. 56-71.

Bremerich-Vos, Albert/Böhme, Katrin (2009): Lesekompetenzdiagnostik - die Entwicklung eines Kompetenzstufenmodells für den Bereich Lesen. In: Granzer, Dietlinde et al. (Hrsg): Bildungsstandards Deutsch und Mathematik - Leistungsmessung in der Grundschule, Weinheim: Beltz, S. 219-249. 
Brüggemann, Anne/Bromme, Rainer (2006): Einleitung. In: Dies. (Hrsg.): Entwicklung und Bewertung von anwendungsorientierter Grundlagenforschung in der Psychologie. Rundgespräche und Kolloquien. Berlin: Oldenbourg Akademieverlag, S. 1-10.

Fay, Johanna (2010): Kompetenzfacetten in der Rechtschreibdiagnostik. Rechtschreibleistung im Test und freien Text. In: Didaktik Deutsch. Jg. 16. H. 29, S. 15-36.

Felten, Michael/Stern, Elsbeth (2012): Lernwirksam unterrichten: Im Schulalltag von der Lernforschung profitieren. Berlin: Cornelsen-Scriptor.

Fleck, Ludwik (1980) [1935]: Entstehung und Entwicklung einer wissenschaftlichen Tatsache. Einführung in die Lehre vom Denkstil und Denkkollektiv. Mit einer Einleitung hrsg. v. Lothar Schäfer und Thomas Schnelle. Textidentisch mit der Ausgabe von 1935. Frankfurt/M.: Suhrkamp.

Frederking, Volker (2012): Symmedialer Literaturunterricht. In: Frederking, Volker et al. (Hrsg.): Taschenbuch des Deutschunterrichts. 3 Bde. Bd. 2: Literaturund Mediendidaktik. Baltmannsweiler: Schneider, S. 535-566.

Frederking, Volker (2014): Deutschdidaktik als transdisziplinäre, anwendungsund grundlagenorientierte empirische Wissenschaft. In: Mitteilungen des DGV. Jg.61. H. 2, S. 109-119.

Funke, Reinold (2014): Erstunterricht nach der Methode Lesen durch Schreiben und Ergebnisse schriftsprachlichen Lernens: eine metaanalytische Bestandsaufnahme. In: Didaktik Deutsch. Jg. 19. H. 36, S. 20-41.

Ivo, Hubert: Deutschdidaktik (1999): Die Sprachlichkeit des Menschen als Bildungsaufgabe in der Zeit. Baltmannsweiler: Schneider Hohengehren.

Kepser, Matthis (2013): Deutschdidaktik als eingreifende Kulturwissenschaft. Ein Positionierungsversuch im wissenschaftlichen Feld. In: Didaktik Deutsch. Jg. 19. H. 34, S. 52-68.

Klieme, Eckhard et al. (2003): Zur Entwicklung nationaler Bildungsstandards. Eine Expertise. Bonn BMBF. http://www.bmbf.de/pub/zur_entwicklung_ nationaler_bildungsstandards.pdf. Abgerufen am 15.03.2015.

Köster, Juliane (2012): Plädoyer fürs Triviale. Was im Deutschunterricht gelernt werden soll. http://www.didaktikdeutsch.de/data/_uploaded/vortraege/ Abschiedsvorlesung.pdf. Abgerufen am 15.03.2015.

McElvany, Nele (2008): Förderung von Lesekompetenz im Kontext der Familie. Münster: Waxmann. Hochschulbildungsreport 2020. Lehrerbildung: http://www.hochschulbildungsreport2020.de/handlungsfeld/lehrer-bildung/ fokus/2014.html. Abgerufen am 19.01.2017. 
Miller, Susanne (2012): Methodenpluralismus oder Methodeneklektizismus? Diplomarbeit, Universität Wien. Fakultät für Philosophie und Bildungswissenschaft. http://othes.univie.ac.at/20266/. Abgerufen am 29.11.2016.

Müller, Bettina (2014): Förderung des Leseverständnisses durch Lesestrategien: Eine Interventionsstudie in der Grundschule. Dissertation Universität Kassel. https://kobra.bibliothek.uni-kassel.de/bitstream/urn:nbn:de: hebis:34-2015012347267/5/DissertationBettinaMueller.pdf. Abgerufen am 06.01.2017.

Neumann, Astrid (2012): Blick(e) auf das schulische Schreiben. Erste Ergebnisse aus IMOSS. In: Didaktik Deutsch. 18. Jg., H. 32, S. 63-85.

Ossner, Jakob (1999): Das Profil der Fachdidaktik. Grundzüge einer praktischen Disziplin. In: Radtke, Frank-Olaf (Hrsg.): Lehrerbildung an der Universität. Zur Wissensbasis pädagogischer Professionalität. Frankfurt/M.: GoetheUniversität, S. 23-45. http://www1.uni-frankfurt.de/fb/fb04/schriftenreihe/ inhalt/k2.pdf. Abgerufen am 11.10.2015.

Ossner, Jakob (2001): Elemente eines Denkstils didaktischer Entscheidungen. In: Rosebrock, Cornelia/Fix, Martin (Hrsg.): Tumulte. Deutschdidaktik zwischen den Stühlen. Baltmannsweiler: Schneider Hohengehren, S. 17-33.

Ossner, Jakob (2005): Die Wahrheit ist konkret und bedarf achtsamer Formulierungen. In: Didaktik Deutsch. 11. Jg., H. 19, S. 20-22.

Reinmann, Gabi (2010): Mögliche Wege der Erkenntnis in den Bildungswissenschaften. In: Jüttemann, Gerd/Mack, Wolfgang (Hrsg.): Konkrete Psychologie. Die Gestaltungsanalyse der Handlungswelt. Lengerich: Pabst, S. 237-252.

Reinmann, Gabi (2013): Entwicklung als Forschung? Gedanken zur Verortung und Präzisierung einer entwicklungsorientierten Bildungsforschung. In: Seufert, Sabine/Metzger, Christoph (Hrsg.): Kompetenzentwicklung in unterschiedlichen Lernkulturen. Festschrift für Dieter Euler zum 60. Geburtstag. Paderborn: Eusl-Verlagsgesellschaft, S. 45-60.

Reinmann, Gabi/Sesink, Werner (2011): Entwicklungsorientierte Bildungsforschung (Diskussionspapier). http://gabi-reinmann.de/wp-content/uploads/ 2011/11/Sesink-Reinmann_Entwicklungsforschung_v05_20_11_2011.pdf. Abgerufen am 13.10.2015.

Rosebrock, Cornelia et al. (2010): Förderung der Leseflüssigkeit bei leseschwachen Zwölfährigen. In: Didaktik Deutsch. 16. Jg., H. 28, S. 33-58.

Tenorth, Heinz-Elmar (2012): Forschungsfragen und Reflexionsprobleme - zur Logik fachdidaktischer Analysen. In: Bayrhuber, Horst et al. (Hrsg.): Formate fachdidaktischer Forschung. Empirische Projekte - historische Analysen theoretische Grundlegungen. Münster u. a.: Waxmann, S. 11-27. 
Wieser, Dorothee (2015): Theorie(?)-Praxis-Konstellationen in Lehrerforschung und Lehrerbildung: Fragen an die aktuelle deutschdidaktische Lehrerforschung. In: Bräuer, Christoph/Wieser, Dorothee (Hrsg.): Lehrende im Blick. Empirische Lehrerforschung in der Deutschdidaktik. Wiesbaden: VS, S. 17-34.

Wissenschaftsrat (2012): Perspektiven der Rechtswissenschaft in Deutschland. Situation, Analysen, Empfehlungen. http://www.wissenschaftsrat.de/download/ archiv/2558-12.pdf. Abgerufen am 13.10.2015.

Zabka, Thomas (2002): Theorie des Deutschunterrichts zwischen pädagogischen und philologischen Zielsetzungen. Rezension zu: Cornelia Rosebrock/ Martin Fix (Hrsg.): Tumulte. Deutschdidaktik zwischen den Stühlen. IASL online. http://www.iaslonline.de/index.php?vorgang_id=2508. Abgerufen am 14.02.2015.

http://symposion-deutschdidaktik.de/fileadmin/dateien/downloads/aktivitaeten/ veranstaltungsarchiv/Ausschreibung_und_Sektionstexte_Ludwigsburg_2016. pdf. Abgerufen am 12.10.2015.

http://www.hochschulbildungsreport2020.de/handlungsfeld/lehrer-bildung/ fokus/2014.html. Abgerufen am 12.10.2015. 
Christoph Bräuer - 978-3-631-70204-8

Downloaded from PubFactory at 01/11/2019 10:39:03AM

via free access 


\title{
Abstand durch Nähe - Nähe durch Abstand. Deutschdidaktik als reflexive Wissenschaft Distance through closeness - closeness through distance. German didactics as a reflexive science
}

Andreas Gruschka zugeeignet

\begin{abstract}
The article advocates an approach to fundamental research in subject didactics. The idea is a shift from outcome-orientated research towards research that reconstructs the logic of processes of mediation and appropriation of literature and language. The theory of teaching literature and language will no longer be a normative theory but should be generated through the sequential analysis of classroom interaction.
\end{abstract}

Während das hochgradig strukturierte Denkrahmenmodell von Jakob Ossner (2006) aus der Kompetenzdebatte hervorgeht und fachbezogene Schülerleistungen als Könnensbereiche verortet, entwickle ich hier einen Ansatz, der in Zusammenhang mit dem gegenwärtigen „professional turn“ in der LehrerInnenausbildung steht. Der rekonstruierende Blick auf Unterrichtsprozesse ist sein Hauptmerkmal. Eine umfassende Theorie des Deutschunterrichts lässt sich daraus erst entwickeln, wenn in hinreichendem Maße fachspezifische Fallstrukturgesetzmäßigkeiten bestimmt worden sind, von denen bislang nur Bausteine existieren (vgl. Pflugmacher 2011; 2015; 2016b). Die Realistische Deutschdidaktik betreibt insofern fachdidaktische Grundlagenforschung und entwickelt die Theorie ihres Objekts aus der empirischen Rekonstruktion von Unterricht. Mit ihr werden typische Inszenierungsmuster und Handlungsherausforderungen des Deutschunterrichts sichtbar und benennbar. Diese stehen in einem Spannungsverhältnis zur traditionellen normativen Objekttheorie der Deutschdidaktik. Als Theorie der Normen im Handlungsfeld Deutschunterricht betrachtet die Realistische Deutschdidaktik die Praxis als eine sinnstrukturierte (vgl. Oevermann 2000). Dieses Spannungsverhältnis spiegelt sich in der durchaus widersprüchlichen Aufgabe, angehende Deutschlehrende zugleich zu ExpertInnen der Sache und zu professionell Handelnden - letzteres vermittels der Befähigung zur stellvertretenden Deutung im Unterricht - zu machen (vgl. Oevermann 1996, S. 70-182). In meinem Beitrag werde ich Grundlagen einer Realistischen Deutschdidaktik 
entwickeln und Fallbeispiele heranziehen, die den Gegenstand einer solchen empirisch gehaltvollen Theorie des Deutschunterrichts sichtbar machen.

\section{Immanente Normativität}

Die Realistische Deutschdidaktik ist keine normative Theorie der Praxis, sondern rekonstruiert die in der Praxis geltenden Normen. Sie ist also im Gegensatz zur traditionellen deutschdidaktischen Theorie eine Theorie der Normen der Praxis. Wie hier zu zeigen sein wird, wissen wir bislang recht wenig darüber, welche Normen im Deutschunterricht handlungsleitend sind, dort mitunter aufeinanderprallen. Es geht also nicht um eine Beschreibung, wie Deutschunterricht idealerweise sein soll, nicht um das utopische Moment mithin, sondern um eine Beschreibung, wie er sich im alltäglichen Betrieb entfaltet. Dazu reicht es nicht, die handlungsleitenden Normen der Lehrenden als „implizites Wissen“ oder "pedagogical content knowledge“ außerhalb des Unterrichts zu erfragen, so eindrücklich und diskussionswürdig die vorliegenden Studien auch sind (vgl. Lindow 2013; Scherf 2013). Denn diese Normen sind zwar bewusstseinsfähig, aber nicht bewusstseinspflichtig. Letzteres wäre auch dysfunktional, denn die Normen führen zu routiniertem Handeln, das in der Regel im situativen Handeln aus guten Gründen nicht Gegenstand von Reflexion wird, da dann gerade eben nicht rasch gehandelt werden könnte. Daher ist davon auszugehen, dass Lehrkräfte andere Phänomene als krisenhafte Herausforderungen wahrnehmen als die den Unterricht rekonstruierenden Forschenden. Lehrkräfte haben zwar ein Gespür für einige krisenhafte Unterrichtssituationen, können aber in der Alltagspraxis kaum alle Herausforderungen wahrnehmen, benennen, geschweige denn rekonstruktiv bestimmen. Zumal es als Pendant zu einer LehrerInnenwissensforschung eine SchülerInnenwissensforschung geben müsste, um die blinden Flecken und Herausforderungen der Praxis von Vermittlung und Aneignung zu erkennen. Mein Ansatz beruht darauf, dass sich die Normen und Problembewältigungsschemata aus der sich sequentiell entfaltenden Interaktion mit Hilfe der Objektiven Hermeneutik nachträglich rekonstruieren lassen (vgl. Pflugmacher 2016a).

\section{Guter, schlechter, alltäglicher Deutschunterricht}

Die Realistische Deutschdidaktik richtet ihren Blick bewusst auf alltäglichen Unterricht. Dadurch wird auch solcher Unterricht in seiner Sinnstrukturiertheit erfahrbar, den wir DeutschdidaktikerInnen aus einer lediglich normativen Sicht als problematisch oder misslungen bezeichnen und letztlich als überwindungswürdig ausblenden würden. Dieser Unterricht sollte jedoch nicht voreilig als 
didaktischer Unfall abgetan werden, der nicht würdig genug erscheint, im wissenschaftlichen Sinn verstanden zu werden. Denn er ist genauso wohlstrukturiert und als sinnhafte Gestalt beschreibbar wie vermeintlich gelungener Unterricht und darf keinesfalls bloß als Abweichung von einem Ideal verstanden werden. Der Rede von der Dignität der Praxis vor der Theorie wird hierbei Rechnung getragen. Nicht indem die Praxis geschont wird unter Verweis auf einen zu akzeptierenden Unterschied von Anspruch und Wirklichkeit, von Theorie und Praxis, sondern indem die in der Wirklichkeit angelegten Ansprüche genau bestimmt und die Schwierigkeiten, sie einzulösen, aufgeschlossen werden.

Den alltäglichen Deutschunterricht zum Gegenstand einer deutschdidaktischen Theoriebildung zu machen, lässt sich auch ausbildungsethisch begründen: Wenn die von der akademischen Deutschdidaktik entwickelten idealen Konzepte in der Praxis nicht funktionieren, führen NovizInnen das Misslingen nicht selten auf sich selbst zurück und greifen dann spontan bzw. routiniert auf die altbekannten Muster zurück, die sie aus dem selbst erfahrenen Deutschunterricht von früher her kennen: Die Rückkehr des Verdrängten droht. Gerade deshalb sollten diese Muster im Sinne einer deutschdidaktischen Grundlagenforschung mit äußerster Präzision bestimmt werden. So gilt: Wenn wir uns für Innovation interessieren, wenn wir für eine Verbesserung des Deutschunterrichts eintreten, sollten wir uns besonders mit alltäglichem Deutschunterricht auseinandersetzen. Nicht indem wir ihn hinsichtlich seiner Wirksamkeit messen, sondern indem wir ihn und seine Funktionsweisen, seine Strukturlogik, seine Handlungsmuster erschließen. Ein ausschließlicher Blick auf die Gelingensbedingungen von Deutschunterricht im Sinne von Best practiseForschung unterstellt lediglich, dass Gelingensbedingungen lehrreicher seien als die Betrachtung von Misslingensbedingungen. Das aber ist keineswegs gesichert, verkauft sich allerdings besser als Andreas Gruschkas - mündlich überlieferte nüchterne Antwort auf die allzu werbewirksame currente Frage, was guter Unterricht sei: „Ganz einfach: die Vermeidung von schlechtem!“ Dazu bedarf es aber der Bereitschaft und der Anstrengung, Unterrichtsprozesse nicht allein an ihrem Outcome zu evaluieren. (Dys)-Funktionalität zeigt sich vielmehr in pädagogischen Sinnstrukturen, wenn man Unterricht an den mit dem Gegenstand oder über die Aufgabenstellungen angesetzten Verstehenspotenzialen misst, also an immanenten Normen und nicht an von außen herangetragenen.

\section{Normentrias}

Eine Studentin fragte mich jüngst, als ich zum wiederholten Male auf die Bedeutsamkeit des Spannungsverhältnisses von Vermittlung und Aneignung verwies, ob die beiden Begriffe nicht für das gleiche stünden. Sie bekam die Antwort: 
„Wenn dem so wäre, bräuchte es keiner Didaktik als Wissenschaft." Die Übereinstimmung von Vermittlung und Aneignung ist eine Idealvorstellung, die einen Ausnahmefall darstellt. Im Normalfall treffen drei Normen aufeinander: die der Lehrenden, die der Lernenden sowie die der Sache. Sie stimmen selten überein. Welche AutorInnen schreiben ihre Texte schon für die Verwendung in der Schule von heute, damit daran der Nachvollzug von Erzählperspektiven eingeübt werde? Die Normen der SchülerInnen können sich darin äußern, dass sie den literarischen Gegenstand als schulischen Gegenstand inszenieren. Oder ihre literarische Rezeption für privat definieren und nicht klassenöffentlich kundtun wollen, weil Aussagen zum Text auch Auskunft über sie selbst sind.

Und im Sinne einer intentio operis gibt es drittens notwendige Verstehensvoraussetzungen der Sache selbst: Texte enthalten Rezeptionshinweise, die das Verständnis regulativ steuern können und in normativer Perspektive steuern sollen. Es wäre zum Beispiel nicht ohne weiteres akzeptabel, den autobiographischen Pakt auf alle Romane anzuwenden, also die Unterscheidung von AutorIn und ErzählerIn aufzuheben. Ebenso wäre es allerdings problematisch, autobiografische Texte als objektive Berichte zu betrachten. Ohne Rücksicht auf Textqualität und -schwierigkeit kann ein Leseakt nicht bewertet bzw. in Hinblick auf etwaige Kompetenzniveaus beschrieben werden (vgl. Pieper 2009, S. 209). Eine Debatte über die notwendigen Verstehensvoraussetzungen als normative Ansprüche des Textes an seinen Idealleser müsste dringend geführt werden. Werden sie erkundet, thematisiert, berücksichtigt? (Wie) Können sie bestimmt werden?

Das Vermittlungshandeln des/der Lehrenden kreist im besseren Fall ständig darum, die unterschiedlichen Normen und Perspektiven abzugleichen. Oft sind es die SchülerInnen, die die Sichtweise der Lehrkraft übernehmen wollen oder sollen. Die SchülerInnen gewinnen dem Gegenstand aber auch Aspekte ab, die der Lehrende nicht intendiert und antizipiert hatte. Das aber muss jener erst einmal erkennen und daraufhin entscheiden, ob er das thematisiert oder dethematisiert (vgl. Pflugmacher 2007). Dies geschieht auch in Praxen der Gruppenarbeit, wenn die SchülerInnen relevante Perspektiven auf den Gegenstand sammeln, bewerten und auswählen. Aus professionstheoretischer Perspektive ist es eine Kernaufgabe des Vermittlungshandelns der bzw. des Lehrenden, sich in die SchülerInnensicht auf den Gegenstand hinein zu fühlen:

- im Vorfeld bei der Planung

- in situ im Unterricht sowie

- nachträglich beispielsweise bei der Auswertung von Rezeptionsdokumenten, wenn die Vermittlungsaufgabe so zur Ermittlungsaufgabe wird. (vgl. Gruschka 2002, S. 121) 
Das ist aber leichter gesagt als getan: Einmal zum routinierten Expertenleser respektive zur Expertenleserin geworden, ist es extrem schwierig, sich in das Nichtverstehen von Lesenovizen einzufühlen. Von Seiten der Deutschdidaktik gibt es dazu m. W. auch wenig Anleitung und Auseinandersetzung. Sowohl der forschende wie auch der Lehrendenblick muss auf die wünschenswerten Erkenntniskrisen der SchülerInnen gerichtet sein, denn "Schule verspricht verstehen" (Combe/Gebhard 2012, S. 7). Geraten die SchülerInnen als Subjekte in eine Krise, wird ihr Ich-Welt-Verhältnis in Frage gestellt und verändert? Das ist schwierig $\mathrm{zu}$ erforschen, entziehen sich Bildungsprozesse als innerpsychische doch dem unmittelbaren Zugang. Dies gilt allerdings sowohl für die bzw. den Lehrenden als auch für die bzw. den Forschenden. Genauso, wie die Lehrkraft Aussagen von Lernenden deuten muss, kann und muss dies - handlungsentlastet umso nachvollziehbarer - auch der bzw. die Forschende. Über die Prozesse der Vermittlung und der Aneignung erhalten wir zumindest indirekt auch Einblicke in die fachlichen Bildungsprozesse der Schülerinnen und Schüler: Über ihre Fragen, ihr Staunen, ihren Widerstand, ihre Ratlosigkeit, ihre Bereitwilligkeit der Aufgabenbearbeitung.

Die handlungsentlastete Arbeit an Unterrichtstranskripten erlaubt auch die Rekonstruktion von Vermittlungskrisen, wenn es also zu Irritation, zu Reibung kommt, die der bzw. dem Lehrenden als nicht wünschenswert erscheint. Der Krisenbegriff ist hier also nicht wie in der Alltagssprache negativ besetzt, sondern er steht zunächst einerseits für eine Wahlsituation von (didaktischen) Handlungsalternativen. Andererseits bezeichnet er die krisenhafte Bewältigung des Ich-WeltVerhältnisses im Sinne von Bildungsprozessen, bei denen bekannte Schemata nicht mehr funktionieren und durch neue ersetzt werden müssen.

\section{Unterrichtsprozessforschung}

Im Folgenden wird gezeigt, welche deutschdidaktisch relevanten Fragen die von mir skizzierte prozessorientierte Forschungspraxis aufwirft oder beantworten kann. „Wie gelernt wird und wie die Logik von Bildungsprozessen zu analysieren ist, ist eine offene Forschungsfrage", stellt Helmuth Feilke in seiner kritischen Bilanz der Output-orientierten Kompetenzforschung fest (Feilke 2014, S. 6). Dabei fokussiert er aber noch immer den Prozess zwischen Sache und Lernenden und erwähnt die bzw. den Vermittelnden nicht. Deutschlehrkräfte treffen aber auch im Unterrichtsprozess didaktische Entscheidungen, sie müssen nachjustieren, Unerwartetem begegnen, etc. Die grundlegende Beziehung zwischen Aneignung/ Kompetenzerwerb und dem Vermittlungshandeln (was nicht auf Methodik verkürzt werden sollte!) wird im psychologisch und nicht didaktisch inspirierten 
Denkrahmenmodell von Ossner nicht aufgeführt. Warum aber sollte dieses Verhältnis nicht theoriewürdig sein? Hier scheint es um eine Grundsatzentscheidung zu gehen: Beschränkt sich die deutschdidaktische Theoriebildung auf die bzw. den Lernenden und die zu erwerbenden Kompetenzen? Als eine ausschließlich am Outcome orientierte Objekttheorie? Oder definiert sie als Objekt ihrer Theoriebildung die Prozesse des Lehrens/der Vermittlung und Lernens/der Aneignung als schulischen Umgang von und mit Sprache und Literatur? Inklusive der Prozesse des (Nicht-)Planens vorweg und des (Nicht-)Nachbereitens? Die nicht allein am vorab definierten erwarteten Outcome erschlossen werden, sondern als literaturpädagogische ${ }^{1}$ Interaktionen?

Damit erweiterte und veränderte sich auch strukturell der Gegenstand der Deutschdidaktik grundlegend. Denn unter dieser Prämisse ginge es fortan auch um die theoretische Beschreibung von Gelingens- und Misslingensprozessen bzw. Gelingens- und Misslingensbedingungen im schulischen Umgang mit Sprache und Literatur. Heiner Willenberg hatte dies diskret angedeutet, als er in seiner Replik auf Ossners Denkrahmenmodellierung schrieb: „[V]iele Lehrer/innen gehen ihrer täglichen Arbeit nach, in der sie immer wieder Beobachtungen gelungener Lernprozesse machen und gleichfalls sehen, dass bei aller Mühe vieles nicht gelingt" (Willenberg 2007, S. 17).

Willenbergs Fazit lautete insgesamt: „Unser Haus der Kompetenzen muss offenbar organisch wachsen, es kann nach keinem fertigen Plan in allen Teilen hergestellt werden“ (ebd.). Diese Ansicht teile ich: Wissen über sprach- und literaturbezogene Lernprozesse, gelingende und misslingende, entsteht in Forschungsprozessen, die bottom-up strukturiert sind und nicht top-down. Über Willenberg hinausgehend ist mein Wunsch, dass deutschdidaktische Theoriebildung auch eine handlungstheoretische Perspektive entwickeln muss. Das hat schon vor langer Zeit in seinen „Deutschkursen“ Harro Müller-Michaels vorgeschlagen und ein Konzept dazu entwickelt (Müller-Michaels 1994). Nachdem seit nunmehr zwei Jahrzehnten die erziehungswissenschaftliche pädagogische Professionsforschung insbesondere mit qualitativ-rekonstruktiven Studien den Unterricht selbst in den Blick nimmt, um die beruflichen Herausforderungen zu bestimmen (vgl. Combe/Helsper 1996), möchte ich beschränkt auf die Deutschdidaktik für einen zweiten Anlauf in diese Richtung argumentieren.

1 Unter Literaturpädagogik verstehe ich nicht außerschulisches professionelles literaturbezogenes Vermittlungshandeln (vgl. Jentgens 2016), sondern eine Weise der Zuwendung zur Lesenovizin bzw. zum Lesenovizen, die sich um die schwierige Einsichtnahme in den individuellen Verstehensprozess bemüht. 
Seit einiger Zeit mache ich die Beobachtung, dass in didaktischen Modellierungen (sowohl von Studierenden als auch in jenen von ExpertInnen) die Kennzeichnung der oben genannten alltäglich und andauernd anzutreffenden Krisen fehlt: Der skizzierte Unterricht funktioniert reibungslos, alternativlos, Unsicherheiten gibt es nicht. Dies zeigt sich sowohl hinsichtlich des Aneignungsprozesses im Sinne eines krisenhaften Erkenntnisgewinns - wir können hier je nach Kontext auch von Konzeptwechsel oder ästhetischer Erfahrung sprechen -, als auch hinsichtlich möglicher Vermittlungskrisen. Bildung wird im Deutschunterricht zumeist inszeniert, als handle es sich um eine Übung. Daraus soll keineswegs gefolgert werden, ein Unterrichtsmodell müsse alle denkbaren Verlaufswege seiner Konkretisierung mitenthalten. Das dürfte eine Überforderung sein. Jedoch sollte ein professionelles Unterrichtsmodell die Zonen benennen, in denen zumindest die wünschenswerten Erkenntniskrisen schülerseits benannt werden. Thomas Zabka hat dies in seinem Text zur didaktischen Analyse literarischer Texte an verschiedenen Stellen in Frageform vorgeführt (Zabka 2012).

In Unterrichtsmodellen bis hin zu jenen in Praxis Deutsch findet man jedoch häufig anstelle einer deutschdidaktischen Argumentation, weshalb die eine Erschließungsphase aus der anderen folgen sollte, satzeinleitende Übergangsformeln wie „Nun kann man“ oder „Hier bietet es sich an“. Zwar kann man sich auf die Position zurückziehen, dass ein Unterrichtsmodell lediglich ein Angebot darstellt, welches gar nicht den Anspruch hat, in exakter Kopie umgesetzt zu werden, da der „Didaktikus“ des Modells ja nicht die konkrete Lerngruppe kennen kann. Der Didaktikus setzt also auf die Umsetzungskompetenzen der erfahrenen AnwenderInnen. Da die Deutschlehrenden jedoch häufig didaktisches Fertigmaterial einsetzen, brauchen wir zumindest für die universitäre Lehre entsprechende Modelle, in denen auch die Irritationspotenziale und die angestrebte krisenhafte Erkenntnis fokussiert werden. Und die findet man nicht in Modellierungen von Deutschunterricht, sondern prima vista durch die Rekonstruktion von tatsächlichem Deutschunterricht - weil sie dort stattfinden oder eben gerade fehlen. Eine deutschdidaktische Theorie, welche die Aneignungsprozesse und Vermittlungsprozesse mit fokussiert, würde also das Defizit der bisherigen Modellierungspraxis kompensieren helfen.

Das Primat der Rekonstruktion vor der Konstruktion sehe ich ganz allgemein darin begründet, dass man auf analytische Weise kennen sollte, was man selbst konstruiert - zumindest kennen können sollte. Sonst folgen die angehenden Deutschlehrkräfte der ihnen in den publizierten Modellen vielfach vorgeführten Kompositionslogik, mit der die didaktischen Entscheidungen eben gerade nicht 
als begründungspflichtige Wahlentscheidungen für und gegen bestimmte Settings sichtbar werden. Während es nachträglich schwierig erscheint, für ein fremdes Modell zu begründen, warum der oder die VerfasserIn den Unterricht genau so und nicht anders strukturiert, lassen sich in der streng methodisch durchgeführten sequenzanalytischen Rekonstruktion von Unterrichtsprozessen die Wahlentscheidungen Schritt für Schritt nachvollziehen und sinnvolle Alternativen gedankenexperimentell bestimmen. Es sind meist gar nicht so viele. Und in einem nächsten Schritt lassen sich die denkbaren sinnvollen Anschlussinterakte bestimmen inklusive ihrer jeweiligen Sinnstruktur. Das sind meist nur einige wenige. Und schon hat man die Entscheidungsstruktur in einer bestimmten sozialen Situation rekonstruiert: In unserem Fall pädagogische Kommunikation, Spezialfall Schulpädagogik bzw. Unterricht, Spezialfall Deutschunterricht. Spezialfall soll heißen, dass im Deutschunterricht vermutlich andere Regeln gelten als in anderen Fächern, und im Literaturunterricht andere als im Sprachunterricht: Zabka (2012) beschreibt die Kompetenz der finalen Ambiguitätstoleranz, die bei der Rezeption literarischer Texte oft hilfreich ist, bei expositorischen Texten hingegen meist keine passende Einstellung zum Text darstellt - beispielsweise bei einer Wegbeschreibung.

\section{Ein Fallbeispiel}

Ein kleines Fallbeispiel ${ }^{2}$ soll illustrieren, dass es schulfachkulturelle Normen gibt und die Normen von Lehrenden und Lernenden divergieren können. Weshalb stellt in Gruschkas Fallanalyse Die Grenzen des Unterrichts (2010) eine Lehrerin die Aufgabe, dass die Schüler Oskar Loerkes Gedicht Blauer Abend in Berlin zweimal, leise, jeder für sich, lesen sollen? Diese Aufgabenformulierung birgt eine bestimmte und bestimmbare Sinnstruktur: Die SchülerInnen sollen sich einer Krise aussetzen und diese zunächst aushalten lernen und durch die zweite, hermeneutische Lektüre bereits erste Lösungen für die Verstehenskrise selbst entwickeln. Die krisenhaft gesteuerte zweite Lektüre wird von der Lehrerin bereits vorstrukturierend eingefordert und nicht den SchülerInnen als selbst zu wählende Lesestrategie überlassen: eine Didaktik der Hilfe zur Selbsthilfe. In der Aufgabenstellung wird die Strategie zur Problembewältigung mitgeliefert, ohne dass das Problemfeld benannt wird. Was für uns DeutschdidaktikerInnen ganz normal und selbstverständlich erscheint, würde in anderen sozialen Kontexten zu Irritationen und potentiellen Nachfragen führen: „Frau Müller, warum sollen

2 Der Fall wird ausführlich sequenzanalytisch rekonstruiert in Pflugmacher 2016b. 
wir denn den Text über die verschiedenen Schichten des tropischen Regenwalds zweimal lesen?" Und gerade indem man dies gedankenexperimentell macht, kann man dann auch die besondere Logik einer solchen Aufgabe im Deutschunterricht verstehen. Hier können die Schüler darauf vertrauen, dass die Lehrerin sich dabei etwas gedacht hat. Sie fragen eben nicht nach: „Und was passiert, wenn ich das Gedicht nur einmal lese?"

Ich bleibe bei dem Fallbeispiel. Es folgt der Dialog zwischen der Lehrerin und einer Schülerin nach der Lesephase, an die kein Arbeitsauftrag geknüpft war (Transkript Nr. 02414 im Archiv für pädagogische Kasuistik (Apaek)).

Lehrerin: Kannst Du mal Deine Frage bitte laut für alle wiederholen?

Schülerin 2: Nö, also ich hab eigentlich nur hier unten bei diesem Eins und Zwei geguckt.

Lehrerin: Ja, aber Du hast doch eben ,ne Frage gestellt, bevor Du nochmal reingeguckt hast.

Schülerin 2: Ja, ich versteh das nicht so ganz.

Schüler ?: Ja, ich auch nicht.

Lehrerin: Du hast gesagt: Verstehst Du das?

Schülerin 2: Ja.

Anstelle einer vollständigen Rekonstruktion dieser Sequenz formuliere ich Fragen, die auf die Besonderheit dieser Interaktion eingehen, welche aus meiner Sicht ein Lehrbeispiel literaturdidaktischer oder vielmehr literaturpädagogischer Empathie darstellt:

- Wie kann (und wie muss) die Lehrerin damit umgehen, dass eine Schülerin sich nicht an die Aufgabe hält und anfängt mit ihrer Mitschülerin zu sprechen?

- Welches sinnvolle Motiv könnte es geben, dass die Schülerin die Wiederholung ihrer Frage verweigert?

- Was bedeutet es, dass die Lehrerin mehrfach darauf beharrt, dass die Schülerin eine Frage hatte?

- Welche sinnvollen Gründe könnte es geben, dass die Schülerin ihre Frage aber nicht öffentlich wiederholen möchte?

- Was bedeutet es, dass die Schülerin nur lokale Verständnisprobleme äußert und das Gedicht allenfalls „nicht so ganz“ versteht?

- Warum wiederholt die Lehrerin die Frage der Schülerin erst am Schluss klassenöffentlich?

- Und was erwartet eine Schülerin eigentlich, wenn sie ihre Nachbarin fragt: „Verstehst Du das?“

Alle diese Fragen weisen auf Interaktionsnormen hin, die nicht sogleich schulfachspezifisch erscheinen mögen. Aber eine gegenstandsindifferente Analyse könnte die Struktur dieser Kommunikation nicht hinreichend aufschließen. Re- 
konstruiert man diesen Fall, wird sichtbar, wie genau eines zum anderen passt, als hätte die Lehrerin das Schülerverhalten geradezu antizipiert. Es treten hervor: Die Peinlichkeit und vermeintliche Privatsache des Nichtverstehens aus SchülerInnensicht sowie eine übergreifende Norm des Verstehens aus Sicht der Lehrerin. Die Lehrerin entfaltet konsequent ihren Unterricht als einen Schutzraum, indem auch Nichtverstehen geäußert werden darf, sogar geäußert werden soll. Sie vermeidet es aber, diesen Schutzraum explizit zu benennen und arbeitet darauf hin, dass die Schülerin selbst die Frage wiederholt - was ihr am Ende nicht ganz gelingt. Der Fall zeigt eine Bearbeitungsmöglichkeit eines zentralen Handlungsproblems der Literaturvermittlung auf: Dass die Lehrkraft das Nichtverstehen der SchülerInnen sichtbar werden lassen muss, wenn daran gearbeitet werden soll - um zum Beispiel Strategien zu entwickeln, es zu reduzieren.

Als Theorie der Praxis rekonstruiert die Realistische Deutschdidaktik die immanenten Normen des Deutschunterrichts als Normentrias. Der Loerke-Fall zeigt dabei, wie die Normen der Schülerin und die Normen der Lehrerin widerstreiten. Die zuvor zitierte Aufgabe zum Loerke-Gedicht dürfte nur schwerlich in einem anderen Fachunterricht zu finden sein bzw. würde dort zu anderen Reaktionsmustern führen. Und auch die Frage aus einem anderen Unterrichtstranskript, wie einem denn die Lektüre gefallen habe, gepaart mit dem Hinweis, die Lehrkraft werde noch an den Eindrücken der SchülerInnen arbeiten, kann man sich in kaum einem anderen Fach vorstellen (vgl. Pflugmacher 2011). Deutschunterricht hat seine eigenen Regeln und Normen, und die Beteiligten halten sich oft daran. Klar ist jedoch, dass die schulfachspezifischen Normen auch erst einmal wie in einem Bildungsprozess erworben werden müssen.

Vorleseunterricht auch in höheren Klassen dient zwar einer kompensatorischen Leseförderung, aber ein aufgabenfreier Deutschunterricht irritiert SchülerInnen, weil er z. B. gegen das Versprechen auf Erkenntnisgewinn verstößt: „Was sollen wir denn jetzt machen?", fragt ein Schüler der 8. Klasse seine Lehrkraft, als diese eine spannende Geschichte vorliest, darauf wartend, dass ihre SchülerInnen endlich - naturwüchsig, intrinsisch motiviert - Fragen stellen. In einem anderen Fall verweigert die Lehrerin nach der Lektüre einer Kurzgeschichte die Aufgabenformulierung und sagt, jetzt machen wir es mal umgekehrt, stellt ihr mal die Fragen - als Ausnahme von der Regel, wie es schon in der Formulierung sichtbar wird. Die SchülerInnen sind völlig irritiert und fragen gar, ob die Fragen denn im Text stehen, die sie nun finden sollen. Die Lehrerin erwartet hier, dass der Text als ein ästhetischer Gegenstand auch ohne Vermittlungsinstanz fragwürdig erscheint, während die SchülerInnen vom Text als Schulgegenstand erwarten, dass er durch Fragestellungen gerahmt wird. Im 
Anschluss bemühen sich die SchülerInnen, wunschgemäß solche Fragen zu (er)finden, die die Lehrerin stellen könnte. Anstatt eine innere Fragehaltung zu aktivieren, versuchen sie sich in die Lehrerin hineinzuversetzen: Literaturpädagogische Empathie oder stellvertretende Deutung, nur in umgekehrter Richtung.

Viele dieser Normen, die im Verlauf der Schulzeit verinnerlicht werden, kennen wir. Thomas Zabka (2012) hat im zitierten Aufsatz auf die finale Ambiguitätstoleranz hingewiesen: Während ein expositorischer Text in der Regel als misslungen gilt, wenn er am Ende mehrdeutig und unbestimmt ist, wäre eine solche Einschätzung hinsichtlich vieler literarischer Texte ein unpassendes Urteil. Gleichwohl müssen diese Regeln erst einmal durchgesetzt werden, explizit oder implizit. Deshalb wäre zentraler Gegenstand einer Realistischen Deutschdidaktik die Bestimmung der im Deutschunterricht wirkenden Normen. Als eine reflexive Theorie gewinnt sie die Beschreibung des Gegenstands erst aus der Rekonstruktion seiner Strukturgesetzmäßigkeiten. Es geht dabei nicht allein um die Frage, welche Normen in ihm gelten, sondern um ihr Zusammenspiel. Denn wir haben es im Deutschunterricht (mindestens) mit einer Normentrias zu tun. So könnte eine Lehrkraft beispielsweise den literarischen Text verzwecken, ihn benutzen als Steinbruch oder Trainingsgelände für die Bestimmung von stilistischen Mitteln, der Erzählstruktur etc., ohne dass dies zu einer Bereicherung der Erschließung des einzelnen Textes führt: Literaturwissenschaftsdidaktik statt Literaturdidaktik nenne ich ein solches Unterrichtsmuster, welches mir in meinen Analysen von Literaturunterricht erstaunlich oft begegnet. Dafür ist der Text nicht geschrieben worden, der Lehrer verstößt damit gegen Ästhetikkonventionen. Und die SchülerInnen könnten sich vom Text jenseits der gestellten Aufgabe irritieren lassen und dies im Unterricht sichtbar machen. Das soll zumindest gelegentlich vorkommen. Die Lehrkraft muss dann situativ zumindest damit umgehen: Soll sie das aufgreifen oder dethematisieren? SchülerInnen könnten den literarischen Text auch, statt an der von der Lehrkraft vorgegebenen Stelle innezuhalten und eine Aufgabe zu bearbeiten, ganz eigensinnig weiterlesen, versunken in die Textwelt, vergessend, dass sie in der Schule sind, wo Texte gemeinhin gemeinsam erschlossen werden und die Unterhaltungsfunktion von Literatur eine randständige Rolle hat. Oder der Lehrende erfragt Leseeindrücke, setzt also auf subjektive Entfaltung, und die SchülerInnen setzen sich zusammen und formulieren gemeinsam Stichworte, da sie der Lehrkraft nicht glauben mögen, dass ihre subjektiven Einschätzungen im Unterricht eine Rolle spielen könnten, wo es sonst um "Sprache und Handlung" geht, wie sie es im Gruppengespräch formulieren (Pflugmacher 2011, S. 130). Ein Schüler 
hat als Hausaufgabe einen Text zu einem literarischen Text geschrieben, in welchem er eine dort aufgeworfene Frage beantwortet. Ein Gespräch über die (nicht explizierten) Weltmodelle von Schüler und Lehrerin entsteht, es geht hin und her. Der Schüler erwartet, dass sie sich in ihn hineinversetzt und seine Perspektive nachvollzieht. Die Lehrerin erwartet hingegen vom Schüler, dass er ihr Verständnis teilt und tut so, als wäre er eigentlich nur vom rechten Weg des Verstehens abgekommen (vgl. Pflugmacher 2015). All das sind tagtägliche Herausforderungen der Literaturvermittlung, die wir aber bislang nicht in unserer Objekttheorie aufgenommen haben. Es dürfte eine überschaubare Anzahl von solchen Konstellationen geben, die sich beschreiben lassen als Dilemmasituationen oder als typische Kunstfehler. Typisch, weil man ihnen immer wieder begegnet. Der Fall, dass die Normen von Lehrkraft und Lernenden und Gegenstand völlig deckungsgleich sind, dürfte eher ein seltener Idealfall sein: Was für soziale Interaktion insgesamt gilt, dürfte auch im Deutschunterricht nicht anders sein. Intention und Wirkung eines Sprechakts sind nicht deckungsgleich. Als Störfaktor treten möglicherweise auch Bezugsnormen aus anderen Fächern auf: So kann man durchaus den Fall antreffen, dass der Lehrende heterogene Ergebnisse erwartet, aber die SchülerInnen aus anderem Unterricht gewohnt sind, dass es ein richtiges Ergebnis geben muss, an dem sie ihre eigene Leistung vergleichend messen wollen.

Keineswegs möchte ich mit der Realistischen Deutschdidaktik die bisherigen Wege der Theoriebildung in der Deutschdidaktik ersetzen. Wir brauchen Idealbeschreibungen oder Utopien. Wir brauchen aber auch eine Auseinandersetzung darüber und Erklärungen dafür, weshalb der alltägliche Deutschunterricht so anders ist, als wir ihn als idealen beschreiben und konstruieren. Dazu bedarf es zunächst einer Theorie der Praxis, die als Strukturtheorie Stück für Stück entwickelt werden kann.

\section{Deutschdidaktische Grundlagenforschung}

Dass ein Denkrahmen dabei sogar hilfreich sein könnte, bestimmte Problembereiche gezielt in den Fokus zu nehmen, will ich gar nicht bezweifeln. Allerdings habe ich in meinen Pilotstudien zu einer Realistischen Literaturdidaktik den gegenteiligen Weg beschritten und schlicht Unterrichtstranskripte von Literaturunterricht analysiert, wie sie mir gerade begegneten. Gerade durch die damit verbundene Neugier und Unvoreingenommenheit war ich in der Lage, bestimmte Probleme der Literaturvermittlung zu erkennen, die ich ausgehend von einer gezielten Fragestellung vermutlich gar nicht wahrgenommen hätte. Um herauszufinden, wie das scheinbar Vertraute funktioniert, muss man es sich fremd 
machen und beispielsweise fragen: Was macht der Lehrer eigentlich, wenn er seinen SchülerInnen einfach vorliest, oder wenn er eine Figurencharakterisierung beauftragt, die zur Formulierung von Stichworten führt? Oder wenn die Lehrerin darauf hinweist, dass man noch ganz lange an diesem Gedicht arbeiten werde oder wenn der Lehrer einen „ersten Einstieg in den Text“ zu schaffen verspricht und daraufhin „an den Eindrücken noch etwas arbeiten“ will (vgl. Pflugmacher 2011)? In jedem Fall setzt sie bzw. er einen normativen Rahmen für sein/ihr eigenes und das Handeln der SchülerInnen, der sich bestimmen lässt. Es könnte durchaus sein, dass gerade die von der Deutschdidaktik entwickelte Begrifflichkeit den Blick verstellt auf Phänomene, die wir dringend erkennen und durchdringen müssten.

Der Anspruch an eine deutschdidaktische Theorie ist aus meiner Sicht auch, die Differenz zwischen Theorie und Praxis, zwischen Sollen und Sein aufzuklären. Das ist der alte Anspruch der Kritischen Theorie. Aber nicht mehr in dem Sinne, dass die Praxis sich zu rechtfertigen habe, weshalb sie der Theorie, dem Ideal hinterherhinke. Diese Projekte sind bekanntlich auch auf anderen Ebenen gescheitert. Der (selbst-)kritische Anspruch besteht eher darin zu fragen, weshalb die Theorie des Deutschunterrichts so wenig zu tun hat mit dem, was dort tagtäglich geschieht: Es fällt recht schwer bzw. ist mitunter unmöglich, den realen Deutschunterricht unter Bezugnahme auf deutschdidaktische Modelle und Terminologien erschließend und nicht bloß als defizitär zu beschreiben. Warum aber sollte die universitäre Deutschdidaktik auf die kritisch-aufschließende Analyse von alltäglichem Unterricht verzichten und diesen Zugang den FachleiterInnen der zweiten Phase überlassen, deren Kritikmonopol ganz massiv mit institutionellen Rahmenbedingungen und einem Bewertungsmonopol verbunden ist, das, zumindest so mein Eindruck, zugleich von vielen Betroffenen als undurchschaubar und subjektiv wahrgenommen wird? Wir verfügen nunmehr über forschungsmethodische Verfahren, die Komplexität der inneren Normativität des Deutschunterrichts zu entfalten und Stück für Stück als Beiträge zu einer Theoriebildung anzunehmen. Dabei sind wir angewiesen auf die gar nicht selbstverständliche Bereitschaft der PraktikerInnen, ihren Unterricht exemplarisch aufnehmen und analysieren zu lassen. In jedem Einzelfall treten immer auch allgemeine Strukturmerkmale hervor. Dafür sollten wir den Deutschlehrkräften nachhaltig dankbar sein.

Will man also nicht weiterhin allein auf das innovationslogische Modell einer Deutschdidaktik setzen, bleibt uns gar nichts anderes übrig, als unsere Hausaufgaben zu erledigen und uns mit den weniger geliebten "Schmuddelecken“ des Deutschunterrichts neugierig auseinanderzusetzen. Eine offene Frage ist für mich im Anschluss daran, wie wir die Theorie der Praxis mit der Theorie für die Praxis in einen produktiven Dialog bringen könnten, ohne dass es in wechselseitige 
Besserwisserei ausartet. Die Theorie der Praxis ist sicherlich in mancher Hinsicht eine Provokation der Theorie für die Praxis, weil sie das Wünschenswerte in Frage stellt, weil sie Fragen aufwirft, die wir (noch) nicht beantworten können, und weil sie längst bekannte, aber gerne verdrängte Befunde aufwirft. Die Debatte dazu erscheint mir überfällig, allerdings sollte die Realistische Deutschdidaktik dann mit mehr Fallanalysen alltäglichen Deutschunterrichts aufgestellt sein, als es bislang der Fall ist.

Kaspar Spinner hat 2006 die 11 Dimensionen literarischen Lernens formuliert. Damit wollte er die Bildungsstandards herausfordern und zeigen, dass es im Literaturunterricht um mehr geht als um allgemeine Kompetenzen. Mittlerweile sind seine Dimensionen in die Bildungsstandards einbezogen worden. Ich schlage daran angelehnt vor, an den 33-66-99 Standardsituationen der Deutschdidaktik in kasuistischer Perspektive zu arbeiten, um die typischen Handlungsprobleme unseres Faches bestimmen zu können. Kolleginnen und Kollegen der Deutschdidaktik sind eingeladen, sich künftig in Göttinger Forschungsworkshops und Theorietagungen an dieser Arbeit zu beteiligen. Abschließend sei daher der paradox anmutende Titel meines Beitrags erläutert: Je näher man sich mikrologisch Deutschunterricht anschaut, umso mehr Abstand entwickelt man zur deutschdidaktischen Theorie. Je mehr man mit fremdem Blick auf die Unterrichtsprobleme im Fach Deutsch schaut, umso näher ist man an den tatsächlichen Handlungsproblemen des Deutschunterrichts dran. Dies ist nicht weniger und nicht mehr als ein Plädoyer für fachdidaktische Grundlagenforschung.

\section{Literatur}

Combe, Arno/Gebhard, Ulrich (2012): Verstehen im Unterricht. Die Rolle von Phantasie und Erfahrung. Wiesbaden: VS Verlag.

Combe, Arno/Helsper, Werner (Hrsg.) (1996): Pädagogische Professionalität. Untersuchungen zum Typus pädagogischen Handelns. Frankfurt/Main: Suhrkamp.

Feilke, Helmuth (2014): „Überarbeiten! Überlegungen zu Bildungsstandards, Textkompetenz und Schreiben." In: Didaktik Deutsch. 19. Jg. H. 37, S. 6-9.

Gruschka, Andreas (2010): An den Grenzen des Unterrichts. Opladen: Barbara Budrich.

Gruschka, Andreas (2002): Didaktik. Das Kreuz mit der Vermittlung. Elf Einsprüche gegen den didaktischen Betrieb. Wetzlar: Büchse der Pandora.

Jentgens, Stephanie (2016): Lehrbuch Literaturpädagogik. Weinheim: Beltz.

Lindow, Ina (2013): Literaturunterricht als Fall: Kasuistisches Wissen von Deutschlehrenden. Wiesbaden: VS Verlag. 
Müller-Michaels, Harro (1994): Deutschkurse. Modell und Erprobung angewandter Germanistik in der gymnasialen Oberstufe. Weinheim: Beltz Athenäum.

Oevermann, Ulrich (1996): Skizze einer revidierten Theorie professionalisierten Handelns. In: Combe, Arno/Helsper, Werner (Hrsg.): Pädagogische Professionalität. Untersuchungen zum Typus pädagogischen Handelns. Frankfurt/ Main: Suhrkamp, S. 70-182.

Oevermann, Ulrich (2000): Die Methode der Fallrekonstruktion in der Grundlagenforschung sowie der klinischen und pädagogischen Praxis. In: Kraimer, Klaus/Garz, Detlef (Hrsg.): Die Fallrekonstruktion. Frankfurt/Main: Suhrkamp, S. 58-156.

Ossner, Jakob (2006): Kompetenzen und Kompetenzmodelle im Deutschunterricht. In: Didaktik Deutsch. Jg. 12. H. 21, S. 5-19.

Pflugmacher, Torsten (2016a): Objektive Hermeneutik. In: Boelmann, Jan (Hrsg.): Empirische Bildungsforschung in der Deutschdidaktik - Erhebungs- und Auswertungsverfahren. Baltmannsweiler: Schneider Hohengehren, S. 285-304.

Pflugmacher, Torsten (2016b): Sequenzanalytische Unterrichtsrekonstruktion als literaturdidaktische Grundlagenforschung. Eine Fallanalyse zur literaturpädagogischen Empathie im Umgang mit literarischer Textschwierigkeit im Literaturunterricht. In: Dawidowski, Christian/Hoffmann, Anna R. (Hrsg.): LehrerInnen- und Unterrichtsforschung in der Literaturdidaktik. Frankfurt am Main: Lang.

Pflugmacher, Torsten (2015): Verstehen verstehen - verstehen. Literaturpädagogische Professionalität als Herausforderung von Literaturdidaktik und Literaturvermittlung. In: Bräuer, Christoph/Wieser, Dorothee (Hrsg.): Die Lehrkraft im Blick - empirische Lehrerforschung in der Deutschdidaktik. Wiesbaden: VS Verlag, S. 129-156.

Pflugmacher, Torsten (2011): ,Und vor allen Dingen an Euren Eindrücken auch ein bisschen arbeiten.' Eine Fallrekonstruktion zu ästhetischer Erfahrung im Literaturunterricht. In: Kirschenmann, Johannes/Spinner, Kaspar H./Richter, Christian (Hrsg.): Reden über Kunst. München: Kopaed, S. 121-135.

Pflugmacher, Torsten (2007): Try Pattern and Drill Error. Zwei Fallanalysen zur Fast-Food-Didaktik mit didaktischen Fertigmaterialien. In: Pädagogische Korrespondenz. Zeitschrift für kritische Zeitdiagnostik in Pädagogik und Gesellschaft. H. 37, S. 81-106.

Pieper, Irene (2009): Literarische Kompetenz: Zentrum oder Peripherie der Kompetenzdiskussion. In: Hochreiter, Susanne et al. (Hrsg.): Schnittstellen. Aspekte der Literatur- und -lernforschung. Innsbruck: Studien Verlag, S. 205-221.

Scherf, Daniel (2013): Leseförderung aus Lehrersicht. Eine qualitativ-empirische Untersuchung professionellen Wissens. Wiesbaden: VS Verlag 2013. 
Spinner, Kaspar H. (2006): Literarisches Lernen. In: Praxis Deutsch 200(2006), S. 6-16.

Willenberg, Heiner (2007): Die Kompetenztheorie für den Deutschunterricht baut noch kein Haus. In: Didaktik Deutsch. 13. Jg., H. 22, 13-22.

Zabka, Thomas (2012): Didaktische Analyse literarischer Texte. Theoretische Überlegungen zu einer Lehrerkompetenz. In: Frickel, Daniela/Kammler, Clemens/Rupp, Gerhard (Hrsg.): Literaturdidaktik im Zeichen von Kompetenzorientierung und Empirie. Fillibach: Freiburg, S. 139-164. 


\title{
„Die Sprachlichkeit des Menschen als Bildungsaufgabe in der Zeit“ - und als Denkrahmen für die Deutschdidaktik
}

\section{'Human language as an educational challenge over time' - and as a theoretical framework for German didactics}

\begin{abstract}
Drawing on Humboldt's and Coserius' ideas about language, this paper uses Hubert Ivo's notion of "human language as an educational challenge over time" to propose an alternative theoretical framework for the didactics of German. Challenging current approaches in the field, Steinbrenner discusses nine aspects of how to teach language and literature in the light of the "freedom of poetic speech" (Jürgen Trabant).
\end{abstract}

\section{Vorgehen}

Der folgende Beitrag umfasst fünf Teile: Im ersten Teil werde ich darstellen, wie die Sprachlichkeit des Menschen als Bildungsaufgabe bei Wilhelm von Humboldt begründet, wie sie von Hubert Ivo als „Denkrahmen“ für die Deutschdidaktik rezipiert und von Jürgen Trabant als zeitgenössischem Sprachwissenschaftler aktualisiert wird. Ich werde dabei vor allem der Frage nachgehen, welchen Stellenwert Literatur in diesem Denkrahmen einnimmt. Daran anschließend werde ich untersuchen, wie sich dieses Denken von Sprache und Literatur zu aktuellen deutschdidaktischen Theorieansätzen (2.), ausgewählten bildungs- und gesellschaftstheoretischen Entwürfen (3.) und den Sichtweisen von Studierenden und Lehrenden auf Literaturunterricht verhält (4). Im abschließenden fünften Teil werde ich als Conclusio und als Anregung zum Weiterdenken neun Aspekte eines Konzepts sprachlich-literarischer Bildung skizzieren.

\section{Die Sprachlichkeit des Menschen als Bildungsaufgabe}

Der Titel dieses Aufsatzes geht zurück auf Hubert Ivos 1999 erschienene Monographie Deutschdidaktik. Die Sprachlichkeit des Menschen als Bildungsaufgabe in der Zeit. Ivo entwickelt hier Bausteine einer Theorie der Deutschdidaktik, deren Bildungsaufgabe er aus der Sprachlichkeit des Menschen herleitet und im Blick 
auf aktuelle Schuldiskurse auslegt. Wilhelm von Humboldts Sprachdenken bildet dabei den zentralen theoretischen Bezugspunkt. Dieser Versuch einer theoretischen Grundlegung der Deutschdidaktik wurde allerdings kaum rezipiert oder weitergedacht. Nach der Jahrtausendwende wurde infolge des ,Pisa-Schocks', des zunehmenden Aktualitätsdrucks und der steigenden Bedeutung der empirischen Forschung im deutschdidaktischen Diskurs vor allem um die Kompetenzorientierung gerungen, der Ivo eher kritisch gegenübersteht (vgl. Ivo 2000). Jakob Ossner bezieht sich in seinem gleichfalls programmatischen Text Elemente eines Denkstils für didaktische Entscheidungen auf Ivos theoretische Grundlegung und hält fest: „Ivo hat einen ,Denkrahmen' für die Deutschdidaktik gefordert und ihn in ,Deutschdidaktik' formuliert. Darin werden die anthropologischen Grundfeste der Disziplin deutlich“ (Ossner 2001, S. 21). „Ein Denkrahmen begründet die Außenlinien und damit das innere Feld, innerhalb dessen in einer Disziplin gedacht werden sollte. Ethische und anthropologische Überlegungen begründen einen Denkrahmen“ (ebd., S. 29).

Ich kann hier Ivos Vorschlag für einen Denkrahmen nicht im Detail vorstellen, möchte aber seine Denkrichtung veranschaulichen, indem ich zunächst einmal vom Titel seiner Monographie ausgehe: Deutschdidaktik. Die Sprachlichkeit des Menschen als Bildungsaufgabe in der Zeit (Ivo 1999). Diese Formulierung verweist auf eine sprachtheoretische, anthropologische und bildungstheoretische Dimension und zudem auf die Geschichtlichkeit und den Zeitbezug, womit sich Ivo klar gegen essentialistische und naturalistische Auffassungen wendet. Sprachtheorie bzw. -philosophie, Anthropologie, Bildungstheorie und nicht zuletzt die Zeit (die Geschichte, aktuelle Gegenwart und antizipierte Zukunft der Sprachlichkeit) sind die zentralen Bezugspunkte für deutschdidaktische Theoriebildung. Vereinfacht lässt sich folgende These formulieren: Wie wir den Menschen als (zu bildendes und sich bildendes) Sprachwesen denken, beeinflusst maßgeblich unsere deutschdidaktische Theoriebildung. Damit plädiere ich mit Ivo für einen bestimmten Denkrahmen, der selbst theoretisch und immer auch theoretisch fundiert ist - auf welche Bezugstheorien er sich dabei stützen sollte, bleibt selbstverständlich umstritten. Dies bringt die Gefahr des Eklektizismus mit sich, zugleich sind anthropologische, bildungstheoretische und sprachtheoretische Bezugnahmen (erst recht in dieser Kombination) immer mit normativen Setzungen verbunden, was ihnen eine besondere Brisanz verleiht.

Für Hubert Ivo stellt Wilhelm von Humboldts Sprachdenken das Fundament für den von ihm formulierten deutschdidaktischen Denkrahmen dar, nicht zuletzt weil dieser in einer bis heute einzigartigen und uneingeholten Weise Anthropologie, Bildungstheorie und Sprachtheorie gemeinsam gedacht hat. Im Folgenden 
versuche ich, dies in der gebotenen Kürze zu skizzieren, wobei ich vor allem auch Humboldt selbst zu Wort kommen lassen will:

Erst im Individuum erhält die Sprache ihre letzte Bestimmtheit. Keiner denkt bei dem Wort gerade und genau das, was der andre, und die noch so kleine Verschiedenheit zittert, wie ein Kreis im Wasser, durch die ganze Sprache fort. Alles Verstehen ist daher immer zugleich ein Nicht-Verstehen, alle Uebereinstimmung in Gedanken und Gefühlen zugleich ein Auseinandergehen. In der Art, wie sich die Sprache in jedem Individuum modificirt, offenbart sich, ihrer im Vorigen dargestellten Macht gegenüber, eine Gewalt des Menschen über sie. [...] In dem auf ihn eingeübten Einfluß liegt die Gesetzmäßigkeit der Sprache und ihrer Formen, in der aus ihm kommenden Rückwirkung ein Princip der Freiheit. Denn es kann im Menschen etwas aufsteigen, dessen Grund kein Verstand in den vorhergehenden Zuständen aufzufinden vermag; [...] Ist aber auch die Freiheit an sich unbestimmbar und unerklärlich, so lassen sich dennoch vielleicht ihre Gränzen innerhalb eines gewissen ihr allein gewährten Spielraums auffinden; und die Sprachuntersuchung muß die Erscheinung der Freiheit erkennen und ehren, aber auch gleich sorgfältig ihren Gränzen nachspüren. (Humboldt 1998[1830-35], S. 190 f.)

Anthropologie: Humboldt spricht hier mehrmals vom Menschen, sein Denken der Sprache ist stets eng mit einem Denken des Menschen verbunden, was besonders prägnant in seinem Diktum „Der Mensch ist nur Mensch durch Sprache“ (Humboldt, erste Akademierede 1820, zit. nach Trabant 2009, S. 7) zum Ausdruck kommt. Für die Anthropologie und die Bildungstheorie zentral ist die ebenfalls von der Sprache aus gedachte Individualität des Menschen. Die Sprache setzt dem Menschen „Gränzen“, sie übt mit ihrer Gesetzmäßigkeit und ihren Formen Einfluss, ja, Macht auf ihn aus - gleichzeitig eröffnet sie ihm aber auch einen Spielraum, ein Moment von Freiheit, denn „erst im Individuum erhält die Sprache ihre letzte Bestimmtheit“. Hier wie auch an vielen anderen Stellen zeigt sich Humboldts dialektisches, ja antinomisches Denken, was nicht zuletzt auch ein Grund für seine Aktualität ist. Es prägt gleichfalls seine Sprachtheorie und -philosophie, was in diesem Zitat besonders deutlich in der Dialektik von Verstehen und Nichtverstehen, Übereinstimmung und Auseinandergehen, Macht der Sprache über den Menschen und Gewalt des Menschen über sie zum Ausdruck kommt. Für Jürgen Trabant steht die Antinomie, die Spannung zwischen zwei einander widersprechenden und dennoch jeweils gültigen Sprachauffassungen, im Zentrum von Humboldts Sprachdenken: So kann Sprache einerseits gedacht werden als

[...] willkürliches Zeichen, Verweisen auf universelle Begriffe und Sachen in der Wirklichkeit, Bezeichnung von Objektivität [...]. Wir müssen, damit wir in der Welt handelnd zurechtkommen, damit Wissenschaft und Technik erfolgreich auf die Welt zugreifen können, präzise referieren. So muß Sprache in der Wissenschaft funktionieren, und so hätte 
die Philosophie (Logik) die Sprache gern. Andererseits aber ist Sprache [...] eben auch eine jeweils ganz besondere lautlich-kognitive Kreation, eine subjektive Welt-Erfassung, ein historisch-partikularer Blick auf die Welt, der diese in einem ganz besonderen Licht erscheinen läßt (Trabant 2008, S. 177).

Die Verschränkung von Anthropologie, Bildungstheorie und Sprachtheorie bei Humboldt möchte ich an dieser Stelle pointiert in fünf Thesen zusammenfassen, wobei bei einzelnen schon der Bezug zu deutschdidaktischen Fragestellungen deutlich wird, um die es hier ja geht: ${ }^{1}$

- Sprache ist zentral für die Bildung des Menschen („Der Mensch ist nur Mensch durch Sprache")!

- Sprache ist zugleich allgemeine Vorgabe und individuelle Aufgabe: Der Mensch wächst in eine Sprachgemeinschaft hinein, deren individuelles Mitglied er werden muss. Zur sprachlichen Bildung gehört somit einerseits, vorgegebene Normen zu kennen und zu befolgen - andererseits ist damit zugleich die Aufgabe verbunden, sich diese Normen individuell anzueignen, d.h. in ein reflexives Verhältnis zu ihnen treten und sie auch verändern und überschreiten zu können. In diesem Sinn konstituieren „Normierung“ und „Allegorese“ die Sprachlichkeit des Menschen und sind deshalb auch grundlegende Kategorien für deutschdidaktisches Denken (vgl. Ivo 2001). ${ }^{2}$

- Sprache darf nicht nur als (äußerliches) Zeichen/Instrument, sondern muss auch als Welt-Ansicht und bildendes Organ gedacht werden, mit dem der Mensch sich körperlich-sinnlich artikuliert. Dies wendet sich gegen Auffassungen von Sprache, die einseitig pragmatisch, instrumentell und kognitiv geprägt sind. ${ }^{3}$

- Sprache ist ihrem Wesen nach dialogisch. Dialogizität als anthropologische Bestimmung bedingt, dass insbesondere auch Spracherwerbsprozesse als in hohem Maße dialogisch, personal und affektiv geprägt verstanden werden müssen.

- Verschiedenheit und insbesondere Mehrsprachigkeit werden grundsätzlich als Chance und Potenzial betrachtet - dabei wird gleichzeitig die Bedeutung der Muttersprachen und der Volks- bzw. Nationalsprachen als identitätsstiften-

1 Ausführlich hierzu Ivo 1994, 1999 und Steinbrenner 2007.

2 Mit Allegorese ist hier nicht nur eine Praxis der Textdeutung gemeint, sondern zunächst einmal die Praxis öffentlich anders zu reden (von griechisch allos „anders, verschieden“ und agoreuo „eindringlich sprechen, eine öffentliche Aussage machen“).

3 Vgl. hierzu auch die Kritik am Europäischen Referenzrahmen für Sprachen durch Jostes 2004. 
de Sprachgemeinschaften gesehen (insbesondere die beiden letzten Aspekte können hier nur genannt und nicht weiter argumentativ entfaltet werden - ich kann hier nur auf die zahlreichen Schriften von Hubert Ivo und Jürgen Trabant verweisen).

Deutlich wird, dass es sich hier um eine bestimmte Auffassung von Sprache handelt, die sich nicht „,beweisen“ und gleichfalls nicht oder nicht nur aus der Empirie ableiten lässt (wenngleich Humboldt auch umfangreiche empirische Sprachstudien betrieben hat). Es handelt sich um bestimmte, theoretische Auffassungen und Denkweisen von Sprache, die immer auch mit normativen Setzungen verbunden sind („So soll Sprache sein“). Nach Humboldt zeichnet es gerade den Menschen aus und gehört mit zu seinen höchsten und wichtigsten Fähigkeiten, dass er in der Lage ist, Sprache zu denken und dass Sprache eben nicht nur naturgegeben, sondern zugleich aufgegeben ist: Was Sprache ist, ist immer auch dadurch mitbestimmt, wie wir Sprache denken.

Daran anschließend möchte ich jetzt aufzeigen, welchen Stellenwert Literatur bzw. das Poetische bei Humboldt einnimmt und dabei zunächst wieder Humboldt selbst zu Wort kommen lassen:

Denn das Gefallen am articulirt hervorgebrachten Laute giebt demselben Reichthum und Mannigfaltigkeit von Verknüpfungen. [...] Oft entpreßt ihn zwar, wie bei widrigen Empfindungen, die Noth; in anderen Fällen liegt ihm Absicht zum Grunde, indem er lockt, warnt, oder zur Hülfe herbeiruft. Aber er entströmt auch ohne Noth und Absicht, dem frohen Gefühle des Daseins, und nicht bloß der rohen Lust, sondern auch dem zarteren Gefallen am kunstvolleren Schmettern der Töne. Dies Letzte ist das Poetische, [...]. (Humboldt 1998[1830-35], S. 195)

Das Poetische und vor allem sein Ursprung, seine Entstehungsgeschichte, sind geprägt durch Mündlichkeit und Sinnlichkeit, Zweckfreiheit und Freiwilligkeit, Lust und Freude. Diese Momente sind für Humboldts Bestimmung des Poetischen zentral und das Poetische hat wiederum auch eine grundlegend anthropologische Bedeutung:

Die Worte entquellen freiwillig, ohne Noth und Absicht, der Brust, und es mag wohl in keiner Einöde eine wandernde Horde gegeben haben, die nicht schon ihre Lieder besessen hätte. Denn der Mensch, als Thiergattung, ist ein singendes Geschöpf, aber Gedanken mit den Tönen verbindend. (Humboldt 1998 [1830-35], S. 187)

Weil das so ist, so der Berliner Romanist Jürgen Trabant, sind Poesie und Dichtung „nicht das Andere der Sprache, sind sie kein abweichendes sprachliches Handeln, sondern gerade die Sprache selbst. In ihr kommt der Mensch als ,Thiergattung' zu sich selbst, sprechend, das heißt singend, Ideen mit Tönen verbindend, artikulierend, in jeder Äußerung des einzelnen sprechenden 
Menschen, aber am schönsten und freiesten in der Dichtung" (Trabant 2009, S. 90 f.). Die Arbeiten Jürgen Trabants (u. a. 2008; 2009; 2012) sind eines der vielen Beispiele dafür, dass Humboldts Sprachdenken aktuell ist und produktiv rezipiert wird. Das letzte Kapitel seines Grundlagenwerks Was ist Sprache?, das sich stark auf Humboldt stützt und auf das die ganze Argumentation hinausläuft, trägt den Titel „Von der Freiheit des poetischen Sprechens“ (Trabant 2008, S. 276 ff.). Auch hier wendet sich Trabant gegen eine Bestimmung des Poetischen als Abweichung von einem wie auch immer gearteten „normalen“ Sprachgebrauch. Neben Humboldt verweist er dabei auf die Arbeiten von Eugenio Coseriu, der der Tradition Humboldts und des romantischen Sprachdenkens folgend Dichtung als „Ort der Entfaltung der funktionellen Vollkommenheit der Sprache“ denkt, denn

der dichterische Sprachgebrauch ist nicht etwa eine Abweichung vom ,normalen' Sprachgebrauch, genau das Umgekehrte ist der Fall: Alle anderen Modalitäten der Sprache wie z. B. die Alltagssprache oder die Wissenschaftssprache [...] stellen Abweichungen gegenüber der totalen Sprache dar, gegenüber der Sprache schlechthin. Wenn man von Reduktion sprechen darf, so im Fall der verschiedenen Arten des nicht-dichterischen Sprachgebrauchs, denn dort werden viele Sprachfunktionen aufgehoben, entaktualisiert', die beim dichterischen Sprechen vollständig präsent sind (Coseriu 1994, S. 148).

Ich zitiere Coseriu hier so ausführlich, weil er für die folgende Argumentation eine zentrale Rolle spielt. Wohlgemerkt: Literatur, Dichtung, Poesie (die Begriffe müssten strenggenommen noch differenziert werden) können im Anschluss an Humboldt, Coseriu, Ivo, Trabant u. a. als „Ort der funktionellen Vollkommenheit der Sprache" gedacht werden - sie müssen es nicht. Entscheidet man sich aber für (oder gegen) diesen Denkrahmen, so hat dies didaktische Konsequenzen.

Dass Coseriu die poetische Rede als nicht deviant betrachtet und dass sie für ihn das eigentliche Sprechen ist, Sprache in ihrer „funktionalen Fülle“, hat für Trabant aber zunächst und vor allem auch ethische Konsequenzen. So ist der ästhetische Bereich der Sprache das einzige Gebiet, auf dem das Sprechen frei sein kann von Zwängen, „wo Generosität regiert, wo (fast) , anything goes. Und diese Freiheit ist seine ethische Bedeutung. Wo sonst, wenn nicht in poetischer Rede, können Menschen erfahren, was Leben sein könnte und sollte" (Trabant 2008, S. 280) und

deshalb und in diesem neuen Sinn - nicht im Sinne eines irgendwie höheren gesellschaftlichen Standards in einer Gesellschaft voller Zwänge - ist poetisches Sprechen distinktiv: Es ist die Distinktion der Spezies (ebd., S. 294). 


\section{Die Sprachlichkeit des Menschen in deutschdidaktischen Theorieansätzen}

\subsection{Lesesozialisation in der Mediengesellschaft (Norbert Groeben, Bettina Hurrelmann)}

Das DFG-Schwerpunktprogramm Lesesozialisation in der Mediengesellschaft ist kein genuin deutschdidaktischer Theorieansatz, sondern eher ein Ansatz der Grundlagenforschung im Bereich der Lesesozialisation. Für mein Thema ist er aber von Interesse, da eine der zentralen Fragestellungen der Forschungsgruppe um Nobert Groeben und Bettina Hurrelmann die Frage nach den Funktionen des Lesens war und wie diese Funktionen mit den Normen der Gesellschaft zusammenhängen. Am Ausgangspunkt ihres „Forschungsüberblicks“ stellt Norbert Groeben die pointierte Frage:

Ist es dann nicht doch sinnvoll, beim Konzept der Lesekompetenz primär von Informationstexten auszugehen und sich auf die kognitiven Teilkomponenten als Kompetenzdimensionen zu konzentrieren [...]? Und zwar nicht primär aus testökonomischen Gründen, sondern weil diese Konzentration empirisch und normativ am besten den Funktionen entspricht, die das Lesen in unserer Mediengesellschaft auf Dauer haben kann und sollte! Oder geht es gerade darum, bestimmte emotional-motivationale Funktionen, die nur bzw. primär mit dem Lesen verbunden sind [...] zu bewahren? (Groeben 2004, S. 21)

Hinter der Frage steht die vor allem auch mit den PISA-Studien verbundene und inzwischen häufig problematisierte Tendenz, Lesekompetenz auf ihre kognitiven Teilkomponenten, ihren instrumentell-pragmatischen Charakter und damit auch insgesamt eher auf das Lesen von Sach- bzw. Informationstexten zu reduzieren, und zwar aus zwei Gründen: Weil die kognitiven Teilkomponenten testökonomisch besser messbar sind und weil das instrumentell-pragmatische Lesen eindeutig zu den Kompetenzen eines "gesellschaftlich handlungsfähigen Subjekts" (Hurrelmann 2004, S. 282) in der Mediengesellschaft gehört, was man von anderen Arten des Lesens nicht ohne Weiteres behaupten kann. So kommen Norbert Groeben und Bettina Hurrelmann denn auch zu dem Schluss:

Insgesamt wird deutlich, dass die ,Ausstrahlung' des Informationslesens auf manifeste Handlungsorientierungen der Person empirisch besser belegt ist als die entsprechende Wirkung von Fiktionslektüre. Das gibt einem Konzept des Lesens als eines Trainingsfeldes, von dem vor allem Effekte auf kognitiv-instrumentelle und sozialpragmatische Dimensionen gesellschaftlicher Teilhabe zu erwarten sind, die große, zuletzt durch PISA wieder verstärkte Überzeugungskraft. (Groeben/Hurrelmann 2004, S. 452) 
Diesem reduktionistischen Modell setzen Groeben und Hurrelmann allerdings ein „kulturelles Modell für das Lesen“ entgegen, das sie durch eine Trias lesebezogener Bildungsnormen beschreiben, die miteinander in Spannung stehen, dabei handelt es sich

erstens um die aus der Aufklärung stammende Norm rationaler Selbstbestimmung. Damit lassen sich die kognitiv-pragmatischen Folgefunktionen der Informationslektüre wie auf Seiten der Fiktion die Ausbildung des Moralbewusstseins zwanglos verbinden. Zweitens ist die aus der idealistisch-humanistischen Tradition stammende Norm existenzieller Persönlichkeitsbildung durch das Lesen zu berücksichtigen, die vor allem in formellen Bildungszusammenhängen verankert ist. Dieser Zielidee entsprechen die Folgefunktionen auf literarischer Seite von der Imaginationsfähigkeit bis zum kulturellen Gedächtnis. Schließlich ist als dritte Norm die Erlebnis- und Genussorientierung zu nennen, die durch die Medienentwicklung eine beachtliche Verstärkung erfährt und vor allem in der unmittelbaren Funktion der Unterhaltung im Sinne von Entlastungs- und Anregungseffekten ihre Entsprechung findet. Und während sich Erlebnisbedürfnisse und Lebensgenuss durch die Angebote der Bildmedien für die meisten Rezipienten/innen leichter realisieren lassen, ist auch die Gefahr nicht von der Hand zu weisen, dass in der Mediengesellschaft Aspekte existenzieller Persönlichkeitsbildung unter Druck geraten, von denen Beiträge zu einer kognitiv-instrumentellen bzw. ökonomisch reduzierten Rationalität nicht ohne weiteres zu erwarten sind (Groeben/Hurrelmann 2004, S. 452).

Rationale Selbstbestimmung, existenzielle Persönlichkeitsbildung und Erlebnisbzw. Genussorientierung als auf das Lesen bezogene Bildungsnormen - ein auf den ersten Blick sehr weit gefasstes Lesemodell. Auf den zweiten Blick fehlt jedoch ein entscheidender Bereich bzw. ist nur sehr implizit mitgedacht: Der Bereich der sprachlichen Bildung. Dies liegt u. a. daran, dass sprachtheoretische Aspekte bei der Theoriekonstruktion praktisch keine Rolle spielen und dadurch Literatur als Sprache auch nicht in den Blick kommen kann. Bei der Konstruktion und Rekonstruktion der Bildungsnormen wird zwar explizit auf die Aufklärung und v. a. auch die idealistisch-humanistische Tradition Bezug genommen (v. a. in Hurrelmann 2004, S. 283 ff.), diese wird aber einseitig und stark verkürzt rezipiert. Der enge Zusammenhang von Sprache, Literatur und Bildung bei Herder, Schleiermacher, Humboldt u. a. kommt kaum in den Blick und wird für die Rekonstruktion und Aktualisierung der Bildungsnormen für die Gegenwart überhaupt nicht genutzt.

Existenzielle Persönlichkeitsbildung und Erlebnis- und Genussorientierung, die vor allem mit dem literarischen Lesen in Verbindung gebracht werden, sind als Bildungsnormen wegen ihrer schwierigen Überprüfbarkeit und ihrer Spannung $\mathrm{zu}$ herrschenden schulisch-institutionellen und auch gesellschaftlichen Normen ohnehin umstritten. Wird Literatur nicht ausgehend von ihrer Sprachlichkeit gedacht, kommt man schnell zu dem Schluss, dass sich auch diese Bildungsnormen 
mit beliebigen anderen Medien realisieren lassen. Auf diese Weise wird Literatur austauschbar und droht letztlich als Bildungsgegenstand obsolet zu werden. Es bleibt die Frage, ob ein vor allem soziologisch und kulturhistorisch abgeleitetes und letztlich recht unspezifisch konturiertes ,gesellschaftlich handlungsfähiges Subjekt" als Denkrahmen für die Konstruktion von Bildungsnormen für die Deutschdidaktik allein ausreicht. Oder vereinfacht und zugespitzt gefragt: Braucht man literarisches Lesen wirklich, um „gesellschaftlich handlungsfähig“ zu sein?

\subsection{Bildungssprache (Helmuth Feilke)}

Aktuellere Beispiele für die Konstruktion von Sprachlichkeit finden sich in dem von Michael Becker-Mrotzek et al. (2013) herausgegebenen Sammelband Sprache im Fach. Sprachlichkeit und fachliches Lernen. In dem Aufsatz Bildungssprache und Schulsprache am Beispiel literal-argumentativer Kompetenzen von Helmuth Feilke ist folgende Grafik zur Veranschaulichung des Konstrukts „Bildungssprache" abgebildet:

Abb. 1: Schriftsprache-Bildungssprache-Schulsprache (vgl. Feilke 2013, S. 118).

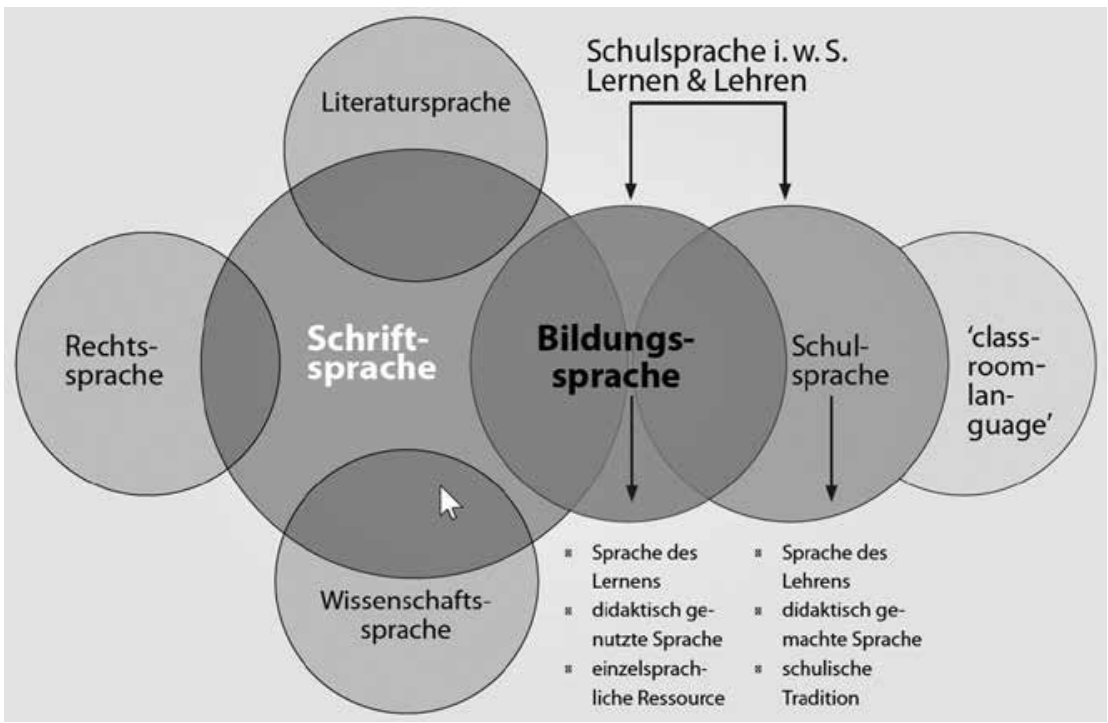

Was auffällt, ist, dass es keinen Überschneidungsbereich zwischen „Literatursprache“ und „Bildungssprache“ gibt. Dies ist sicher keine Absicht und es ist auch nicht der thematische Fokus des Aufsatzes. Ein Zitat weist aber in eine 
ähnliche Richtung: „Die Bildungssprache gehört der Schriftsprache an, so wie Literatursprache, Rechtssprache, Wissenschafts- und Fachsprachen und ggf. auch weitere Varietäten“ (Feilke 2013, S. 119). Steht Literatursprache also auf einer Ebene wie Rechts- oder Wissenschaftssprache, ist sie gar eine Varietät? Die Grafik lässt mehrere Deutungsmöglichkeiten zu, offensichtlich ist jedoch, dass Literatursprache hier anders gedacht wird als bei Trabant und Coseriu. Wenn man den Diskurs um die Bildungssprache in der Deutschdidaktik verfolgt, fällt zudem auf, dass tatsächlich mit Ausnahme von auf die Grundschule bezogenen Arbeiten praktisch kein Bezug auf Literatur/Literatursprache genommen wird. Bildungssprache wird vor allem sprach- und kultursoziologisch gedacht - ein Denkrahmen, der, mit Abstand betrachtet, als einseitig und fremdbestimmt erscheint.

\subsection{Literarästhetische Urteilskompetenz und Kommunikation (Volker Frederking et al.)}

In einem weiteren Aufsatz aus demselben Sammelband mit dem Titel Literarästhetische Kommunikation im Deutschunterricht von Volker Frederking et al. findet sich gleich zu Anfang folgende Begriffsbestimmung: „Poetische Sprache kann als spezifische Ausprägung von Fachsprache verstanden werden" (Frederking et al. 2013, S. 131). Auch dieses Verständnis von poetischer Sprache widerspricht offensichtlich der im ersten Kapitel gezeigten Auffassung, nach der Literatur gerade keine Fachsprache ist, und dies hat hier erhebliche didaktische Konsequenzen.

Eine zentrale Ausgangsfrage der Forschungsgruppe um Frederking lautet: „Was aber macht die Spezifik eines literarischen Textes aus, der [...] literarästhetische Kommunikation konstituiert?“ (Frederking et al. 2013, S. 133). Die Frage wird vor allem mit Roman Jakobson und der semiotischen Ästhetik Umberto Ecos beantwortet, bei der

die ,ästhetische Funktion' als Kern poetischer Sprache ausgewiesen wird. Eine ästhetische Funktion ist dann gegeben, wenn der sprachliche Code zwei- oder mehrdeutig strukturiert ist und autoreflexiv erscheint, d.h. die Aufmerksamkeit auf die eigene Form zu lenken vermag und den Leser dazu bringt, darüber nachzudenken, mit welcher, Textstrategie' ein literarischer Text unterschiedliche semantische Interpretationen evoziert (ebd., S. 133 f.).

Die Angemessenheit von Frederkings Jakobson- und Eco-Interpretation kann an dieser Stelle nicht diskutiert werden. ${ }^{4}$ Auch die Ästhetiken Jakobsons und

4 Coseriu zur „poetischen Funktion“ bei Jakobson: „Wenn Jakobson behauptet, die Einstellung auf die Nachricht als solche, die Zentrierung der Nachricht um ihrer selbst 
Ecos stellen für Trabant jedenfalls eine Form von „Abweichungstheorie“ dar (vgl. Trabant 2008, S. 281 ff.), insofern literarische Texte hier (abweichend von ,normalsprachlichen' Texten) zwei- oder mehrdeutig strukturiert sind und sie (ebenfalls abweichend von ,normalsprachlichen' Texten) die Aufmerksamkeit auf die eigene Form lenken. (Wobei sich auch hier die Frage stellt, was das konkret heißt: Bin ich "aufmerksam“, wenn ich erschreckt, irritiert oder freudig erregt bin, oder wenn ich die formalen Mittel erkennen und benennen kann?)

Vor allem auf dieser theoretischen Grundlage leitet die Forschungsgruppe dann ein dreidimensionales Modell "literarästhetischer Verstehenskompetenz" (in anderen Texten „literarästhetische Urteilskompetenz“) ab, das folgende drei Teildimensionen hat und der Strukturierung literarästhetischer Kommunikationsprozesse dienen soll:

1. Semantische literarästhetische Verstehenskompetenz (die Fähigkeit zum Erschließen zentraler Inhalte $[\ldots])$.

2. Idiolektale literarästhetische Verstehenskompetenz (die Fähigkeit zum Erfassen der formalen Spezifik [...]).

3. Kontextuelle literarästhetische Verstehenskompetenz (die Fähigkeit zum Erfassen außertextlicher Bezüge [...]). (Frederking et al. 2013, S. 140)

Hier scheint auf den ersten Blick ein klassisches Modell des didaktischen Brauchtums im Umgang mit literarischen Texten auf: Erschließen zentraler Inhalte Erfassen der formalen Spezifik - Erfassen außertextlicher Bezüge, wobei ein Schwerpunkt auf dem Erfassen der formalen Spezifik liegt, da dies nach Frederkings Eco-Interpretation zentral für die ästhetische Funktion ist (s.o.) und da die anderen beiden Teildimensionen auch für das Lesen nicht-literarischer Texte relevant sind. Zugespitzt formuliert: Literarische Sprache wird in dieser Modellierung tatsächlich wie eingangs formuliert zu einer „Fachsprache“, bei der es vor allem darum geht, die formale Spezifik zu erfassen, mit der sie von der Alltagssprache oder anderen Fachsprachen abweicht (und erfassen heißt zumeist, sie zu erkennen und zu benennen). Die individuelle Sinndeutung (was sagt der Text mir?), der affektive Bezug (wie wirkt der Text auf mich, was löst er bei mir aus?) und die Wahrnehmung der Klangdimension literarischer Sprache kommen hier kaum vor. Vor allem aber bleibt der Bildungssinn, der hinter diesem Tun steckt, unbestimmt und so wäre vielen SchülerInnen bei einem solchen Vorgehen im

willen sei die ,poetische Funktion' der Sprache, so kann es sich dabei nicht um eine Konzentration auf das Wie des Gesagten, um ein besonderes Raffinement bei der Gestaltung des Mitzuteilenden handeln. Es muß etwas anderes damit gemeint sein: das Verweilen bei dem in der Sprache selbst Gesagten [...]“ (Coseriu 1994, S. 149). 
Unterricht auch unklar, warum sie das eigentlich machen, außer für die Schule, den Test oder für die Prüfung. Die Twitter-Debatte, die die 17-jährige Schülerin Naina um den Sinn und Unsinn der „Gedichtsanalyse" losgetreten hat, passt gut in diesen Kontext (Abbildung 2):

\section{Abb. 2: Der Tweet von Naina ${ }^{5}$}

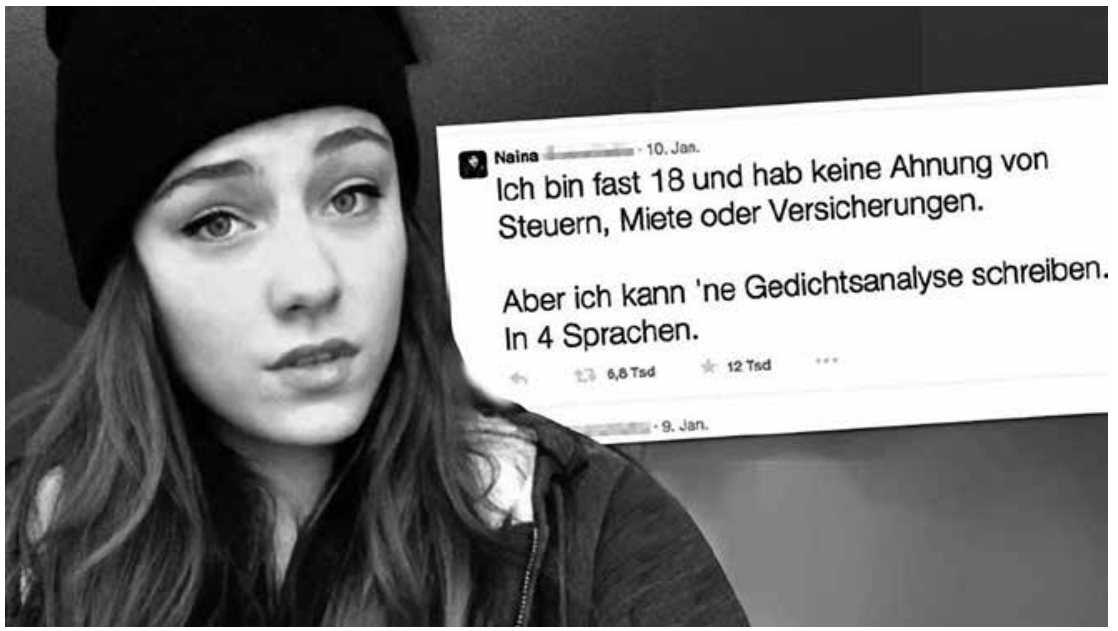

Die Arbeiten der Forschungsgruppe um Frederking sind ein gutes Beispiel dafür, wie die Anforderungen der empirischen Forschung und die Einflüsse des didaktischen Brauchtums zu einem hochelaborierten, aber letztlich stark reduktionistischen Modell „literarästhetischer Kommunikation“ führen. Hubert Ivo versuchte dagegen, das „Reden über poetische Sprachwerke“ als ein „Modell sprachverständiger Intersubjektivität“ zu konturieren, bei der es keine „Gewaltsamkeit des letzten Wortes“ gibt (vgl. Ivo 1994, S. 222 ff.). Dies kann hier nicht ausgeführt werden, die Kernaussage lautet aber: Wenn die „Freiheit des poetischen Sprechens" als Ausgangspunkt genommen wird, dann müsste genau dies auch das Sprechen über poetische Sprachwerke charakterisieren, und zwar als ein nicht-definitorisches, suchendes, abwägendes und tentatives Sprechen, was ich an anderer Stelle als „mimetisches Sprechen“ bestimmt und an Gesprächsbeispielen aufgezeigt habe (vgl. Steinbrenner 2014a).

5 Vgl. dazu den Beitrag und die Kommentare unter: http://www.zeit.de/gesellschaft/ zeitgeschehen/2015-01/twitter-nainablabla-schule-diskussion. Abgerufen am 15.03.2016. 


\subsection{Lesedidaktik (Cornelia Rosebrock und Daniel Nix)}

Die Grundlagen der Lesedidaktik und der systematischen schulischen Leseförderung von Cornelia Rosebrock und Daniel Nix sind der im Moment wohl einflussreichste Ansatz im Bereich der Lesedidaktik. Das Kapitel, in dem es um die spezifischen "Potentiale des literarischen Lesens" geht, beginnt ebenfalls mit einem Versuch, die Spezifik dieses Gegenstands zu erfassen:

Zur definitorischen Abgrenzung von literarischen Texten gegenüber anderen Texten bezieht man sich meist auf zwei Konventionen, innerhalb derer Literatur geschrieben und verstanden wird, und zwar die Polyvalenzkonvention, also das verbreitete Wissen, dass literarische Texte als ganze ebenso wie in ihren Bestandteilen mehrdeutig sind, und die Ästhetikkonvention, also das Gebot, solche Texte zunächst in ihrem eigenen Horizont $\mathrm{zu}$ lesen und nicht sofort auf Referenzen in der Lebenswelt hin zu überprüfen.

(Rosebrock/Nix 2014, S. 142; Herv. i. Orig.)

Rosebrock und Nix verweisen hier vor allem auf die Arbeiten von Siegfried J. Schmidt und man erkennt auf den ersten Blick, dass es sich hier ebenfalls um eine Form von „Abweichungstheorie“ handelt. Abweichend von anderen Texten (bei Rosebrock und Nix wird die Abgrenzung v. a. zu Sachtexten vorgenommen) sind literarische Texte meist mehrdeutig und ebenfalls abweichend von anderen Texten verweisen sie zunächst auf sich selbst. Diese Abweichungen sind durch Konventionen geregelt, die spezielle Anforderungen an die LeserInnen stellen, z.B. größere Unsicherheitstoleranz oder die Fähigkeit zur begleitenden Selbstreflexion. Die Konventionen und die ihnen entsprechenden Einstellungen und Fähigkeiten müssen erlernt bzw. erworben werden - doch warum und wozu? Auch hier stellt sich die Frage nach dem spezifischen (Bildungs-)Sinn des literarischen Lesens, der ja mit dem bloßen Erwerb und der Aneignung von Konventionen kaum erschöpft sein kann. Theorieansätze Schmidt'scher Provenienz können darauf nur bedingt eine Antwort geben.

Rosebrock und Nix versuchen eine Antwort, indem sie unter Verweis auf literaturdidaktische Positionen (v. a. Kaspar H. Spinner) drei „spezifische Leistungen der (Kinder-)Literatur bei der Identitätsbildung“" nennen, und zwar die Herausforderung und Entwicklung der Imaginationsfähigkeit, das Vertrautwerden mit kulturellen Gefühlsmustern und die Ausbildung der moralischen Urteilsfähigkeit (vgl. Rosebrock/Nix 2014, S. 143). Das sind einleuchtende, wichtige und konsensfähige Zielsetzungen - es stellt sich jedoch auch hier die Frage, ob dies wirklich „spezifische Leistungen“ der Literatur sind, und ob mit dem Fokus auf „Identitätsbildung“ nicht die sprachlich-literarische Bildung aus dem Blick gerät. Identitätsbildung im hier beschriebenen Sinn kann und wird jedenfalls 
mit einer Vielzahl von Medien in praktisch allen Unterrichtsfächern angestrebt sprachlich-literarische Bildung dagegen nicht.

Insgesamt werden in dem Konzept von Rosebrock und Nix Lese- und Literaturdidaktik zu wenig und zu unscharf in ihren Gemeinsamkeiten und Unterschieden konturiert. Sicherlich nicht intendiert, besteht dabei die Gefahr, dass die Literaturdidaktik zu einem bloßen Anhängsel der Lesedidaktik wird, mit einem einseitigen Fokus auf der Rezeption, dem Lesen und Verstehen literarischer Texte, verbunden mit dem Ziel, die literaturspezifischen Konventionen erkennen und befolgen zu können, und dem Ziel der Persönlichkeits- oder Identitätsbildung. Die beiden letzten Ziele werden, unspezifisch formuliert, von jedem Schulfach verfolgt.

Auch in dieser Konzeption literarischen Lesens (und Rosebrock und Nix sprechen explizit und ohne Einschränkungen vom literarischen Lesen) werden die Sprachlichkeit literarischer Texte und insbesondere auch deren Klangdimension nicht thematisiert. Bemerkenswert ist, dass diese Klangdimension dann in der Lesedidaktik unter ganz anderen Vorzeichen zum Kern einer neuen Methodik wird: Der innovativste und am breitesten rezipierte Teil der Lesedidaktik sind die „Lautleseverfahren“, bei denen in unterschiedlichen Formen das laute Lesen vor allem literarischer Texte genutzt wird, um die Leseflüssigkeit der SchülerInnen zu messen, zu vergleichen und zu trainieren. Eben weil dieser Ansatz auf messbare Ergebnisse zielt und diese auch erreicht, ist er im Moment so populär. Allerdings sind diese Ergebnisse bislang kurzfristiger Natur, es existieren noch keine Langzeitstudien und ich möchte hier die kritische Frage stellen, welche Vorstellungen von Literatur bei einem solchen Vorgehen nebenbei und unbeabsichtigt vermittelt werden und welche langfristigen Wirkungen (und vielleicht sind es sogar Kollateralschäden) es hat - das ist, wenn überhaupt, dann weit schwieriger zu messen. Dies spricht nicht gegen Lautleseverfahren per se, wohl aber für eine Einbettung in einen Deutschunterricht, in dem das literarische Lesen auch in seiner Klangdimension eine wichtige, gegenstandsangemessene Rolle spielt.

\subsection{Literarisches Lernen (Kaspar H. Spinner)}

Abschließend möchte ich noch auf den wohl wirkungsmächtigsten Text im literaturdidaktischen Diskurs der letzten Jahre eingehen: Kaspar H. Spinners Basisartikel Literarisches Lernen in der Zeitschrift Praxis Deutsch (Spinner 2006). ${ }^{6}$ Ausgangspunkt von Spinner ist die "Auffassung, dass es Lernprozesse gibt, die sich speziell auf die Beschäftigung mit literarischen, das heißt hier: fiktionalen,

6 Vgl. hierzu v. a. die Auseinandersetzung in Leseräume 2/2015. 
poetischen Texten beziehen“ (Spinner 2006, S. 6). Diese Lernprozesse versucht Spinner dann mit seinen 11 Aspekten zu beschreiben. Zwei zentrale Fragen werden dabei jedoch kaum thematisiert:

1. Warum gibt es überhaupt Lernprozesse, die sich speziell auf die Beschäftigung mit literarischen Texten beziehen? Um diese Frage zu klären, müsste der Gegenstand bzw. die Auffassung, die Spinner vom Gegenstand hat, näher bestimmt werden. Dass dies nicht geschieht, zeigt schon die annähernde Gleichsetzung von literarischen, fiktionalen und poetischen Texten im Zitat und die Tatsache, dass sich Spinner im weiteren Verlauf sowohl auf die Hermeneutik, die Dekonstruktion und die Rezeptionsästhetik beruft (wobei er letztere besonders betont, die Differenzen der drei Literaturtheorien jedoch ausblendet).

2. Warum sind solche speziellen Lernprozesse überhaupt sinnvoll? Welchen „Bildungssinn" hat das hier beschriebene literarische Lernen eigentlich und wie kann es sich, und damit literarische Texte als Lerngegenstände, in der Schule legitimieren?

Auf der einen Seite erscheint Spinners Text sehr klar, strukturiert, gut verständlich und anschlussfähig - auf der anderen Seite werden jedoch zentrale Grundfragen nicht explizit und transparent thematisiert, was Michael Baum wie folgt beschreibt:

Die viel zitierte Klarheit des Textes bleibt folglich auf ein Moment der Intransparenz bezogen; die Positivitäten des Textes erweisen sich als Größen, die zu ihrem Anderen, der ,literarischen Kultur', in einem wechselseitig supplementären Verhältnis stehen: Behauptet wird auf der einen Seite, dass die , literarische Kultur' die Vorarbeit der Schule benötigt; auf der anderen Seite braucht der didaktische Text die Referenz auf eine semantisch vage bleibende ,literarische Kultur', um sich als Instruktion für schulisches Lernen legitimieren zu können. (Baum 2015, S. 17)

Ich bin der Auffassung, dass das „Moment der Intransparenz" vor allem auch dadurch entsteht, dass Spinner seinen Denkrahmen - sei es nun „literarische Kultur“ oder eine bestimmte Auffassung von Literatur und Sprache - nicht explizit macht. Dies kann im Rahmen eines Basisartikels für Praxis Deutsch auch nicht geleistet werden (könnte jedoch an anderer Stelle nachgeholt werden. Mit dem Schlagwort Rezeptionsästhetik ist es jedenfalls nicht erledigt).

Bemerkenswert ist, dass gerade dieser Text eine derartige Wirkmächtigkeit im didaktischen Diskurs erlangt hat. Ein Grund dürfte - neben dem Publikationsort, der anschaulichen, auf Konsens abzielenden Sprache und Argumentation und der Mehrfachadressierung - vor allem die hohe Anschlussfähigkeit des Textes sein. Diese entsteht auch durch die hier beschriebene Intransparenz und den 
fehlenden expliziten Denkrahmen, denn vor allem dadurch ist der Text in der Lage, vordergründig die unterschiedlichsten Ebenen anzusprechen und zu vermitteln (u. a. „verschiedene Diskurse der Literaturdidaktik, Elemente der Literaturtheorie, Vorstellungen von Unterrichtspraxis, amtliche Texte wie Lehrpläne und Verlautbarungen der Kultusminister usf.“ (Baum 2015, S. 18)). „Wenn aber“, so Michael Baum, „der Verzicht auf theoretische Fundierung bzw. die vordergründige Vermittelbarkeit aller Ebenen gewollt ist (bei gleichzeitiger wissenschaftlicher Formgebung), dann stellt sich die Frage, in welchem Verhältnis solche Texte zum wissenschaftlichen Diskurs im engeren Sinne stehen“ (Baum 2015, S. 21). Und Baum spitzt seine kritische Lesart mit deutlichem Bezug zu Foucaults Macht- und Diskurstheorie noch zu:

Man kann auch sagen, dass die Funktion der ,elf Aspekte', Handeln zu orientieren, untrennbar mit der Reproduktion von Macht einhergeht. Diese Macht nimmt sich alles, was sie braucht: eine Didaktisierung der Didaktik auf der stilistischen Ebene, eine Unterdrückung der Paradoxien der Lesbarkeit und Verstehbarkeit von Literatur, eine Zurechnung von Wissen auf individuelles Können, eine Subordination unter die Forderungen der Politik, eine Trivialisierung von Theorie. (Baum 2015, S. 26)

Man kann Spinners Text jedoch auch ganz anders lesen und einschätzen, und ich selbst schwanke ständig zwischen beiden Lesarten, was vor allem auch daran liegt, dass ich die Wirkmächtigkeit von Spinners Text im positiven wie im negativen Sinn alltäglich in der literaturdidaktischen Lehre an einer Pädagogischen Hochschule erfahre. Hans Lösener praktiziert eine „andere Lesart“, indem er versucht, zunächst die „Subjektivität beim literarischen Lernen“ als das zentrale Moment in Spinners Konzept zu bestimmen und anschließend zu „präzisieren“. Diese Präzisierung

bedarf einer sprachtheoretischen Reflexion, ohne die es - so meine ich - keine fundierte Konzeption des literarischen Lernens geben kann. So wie jede Konzeption des Literaturunterrichts eine Poetik voraussetzt, muss sie sich ihrer sprachtheoretischen Grundlagen bewusst sein. Fehlt ein solches Bewusstsein, so droht dem literarischen Lernen eine Abkopplung von der Sprachlichkeit, die seine Gegenstände und Arbeitsformen konstituieren. [...] Denn literarisches Lernen versteht sich nicht von selbst. Es braucht eine umfassende Begründung, die insbesondere die Frage nach der Sprachlichkeit des literarischen Lernens einschließt: Was lernen Schülerinnen und Schüler, wenn sie mit Literatur umgehen, was sie sonst nicht lernen würden? (Lösener 2015b, S. 73)

Bei seiner sprachtheoretischen Reflexion bezieht sich Lösener explizit auf Humboldt, Benveniste und Meschonnic (vgl. ebd., S. 76 f.) - ein Denkrahmen, der im Wesentlichen dem hier im ersten Kapitel entworfenen entspricht. Ausgehend von diesem Denkrahmen akzentuiert und präzisiert Lösener dann einzelne As- 
pekte, und zwar vor allem die Aspekte „[s]ubjektive Involviertheit und genaue Wahrnehmung miteinander ins Spiel bringen “ und „[d]ie Sprachliche Gestaltung aufmerksam wahrnehmen“, die er mit seinen Konzepten eines „erfahrungsorientierten Lyrikunterrichts“ und des „hörenden Lesens“ in Verbindung bringt, bei der die Stimmlichkeit der Sprache eine zentrale Rolle spielt (Lösener/Siebauer 2011; Lösener 2015a,b). Von hier aus entwirft Lösener auch „Perspektiven für das sprachethische Lernen“ (Lösener 2015b, S. 81 f.).

Auf diese Weise werden Spinners Aspekte deutlich akzentuiert und präzisiert es stellt sich allerdings die Frage, ob dieser Denkrahmen in gleicher Weise auf alle Aspekte anwendbar ist. Kritisch könnte man einwenden, dass Lösener einfach die Aspekte herausgreift, die in seinen Denkrahmen passen. Vor allem ist offensichtlich, dass Löseners Ansatz weit weniger anschlussfähig ist und in erheblicher Spannung steht zu den Bildungsstandards, einer empiristischen Wissenschaftsauffassung und nicht zuletzt zum didaktischen Brauchtum, was Lösener auch explizit und viel deutlicher als Spinner zur Sprache bringt.

\section{Die Sprachlichkeit des Menschen als Bildungsaufgabe in ausgewählten bildungs- und gesellschaftstheoretischen Entwürfen}

Um die Sprachlichkeit des Menschen als Bildungsaufgabe und den Stellenwert der Literatur noch etwas genauer zu fassen, möchte ich im Folgenden auf einige bildungs- und gesellschaftstheoretische Entwürfe eingehen, die Sprache und Literatur, Anthropologie und Bildung thematisieren, und zwar nicht isoliert, sondern in ihrem inhärenten Zusammenhang. In allen Ansätzen wird, zum Teil mit unterschiedlicher Begrifflichkeit, die Auffassung vertreten, dass der Literatur durch ihre spezifische Sprachlichkeit ein besonderer Bildungswert für den Menschen zukommt.

\subsection{Autorin des eigenen Lebens sein (Peter Bieri, Julian Nida-Rümelin)}

Peter Bieri, zunächst Sprachphilosoph und dann Schriftsteller, bestimmt in seiner Rede Wie wäre es, gebildet zu sein? poetische Erfahrung und Artikuliertheit als zentrale Momente von Bildung. Zur Artikuliertheit führt er aus, dass der Leser von Literatur lernt,

wie man über das Denken, Wollen und Fühlen von Menschen sprechen kann. Er lernt die Sprache der Seele. Er lernt, dass man derselben Sache gegenüber anders empfinden kann, als er es gewohnt ist. Andere Liebe, anderer Hass. Er lernt neue Wörter und neue 
Metaphern für seelisches Geschehen. Er kann, weil sein Wortschatz, sein begriffliches Repertoire, größer geworden ist, nun nuancierter über sein Erleben reden, und das wiederum ermöglicht ihm, differenzierter zu empfinden (Bieri 2008, S. 18).

Auch in seiner Vorlesungsreihe Wie wollen wir leben? nehmen Sprache und Literatur eine wesentliche Rolle ein, was schon an den Zwischentiteln deutlich wird (u. a. Literatur als mächtige Verbündete, Sich schreibend erkennen, Der Schlüssel zu allem: Sprache). Besonders wichtig ist Bieri hier der Gedanke, dass Selbstbestimmung vor allem mit einer „eigenen Stimme“ und mit der Fähigkeit und Möglichkeit zusammenhängt, sich selbst zu erzählen oder genauer: sein Selbstbild erzählerisch zu konstruieren. Was wir dabei „aus Literatur lernen können, ist, wie man sich selbst erzählt" (Bieri 2013, S. 25). Auch Julian Nida-Rümelin beruft sich in seinem Essay Philosophie einer humanen Bildung an zentralen Stellen auf Humboldt. Für ihn ist „Autorin des eigenen Lebens zu sein“ die „aktuelle Botschaft humanistischer Bildungsphilosophie“ (2013, S. 246) - und das heißt, geschult an anderen Autorinnen sein eigenes Leben schreiben (und lesen, erzählen und hören) zu können. In diesen Leitvorstellungen sind sprachliches und literarisches Lernen in eins gedacht. Nida-Rümelin und auch Bieri nehmen gegenüber aktuellen Entwicklungen im Bildungsbereich eine sehr kritische Position ein und problematisieren vor allem eine zunehmende Instrumentalisierung der Bildung für ökonomische Zwecke, die sich nicht zuletzt auch an der Bildungssprache zeigt und an der Sprache, mit der über Bildung gesprochen wird.

\section{2 „Dimension expressive“ und „Dimension stratégique de l'usage de la langue“" (Pierre Judet de la Combe)}

In Frankreich wird ebenfalls und teilweise breiter und öffentlicher ein Diskurs um den Stellenwert der Literatur und auch der alten Sprachen im Unterricht an öffentlichen Schulen geführt. Einflussreich waren hier u.a. die Arbeiten des Gräzisten Pierre Judet de la Combe. Ausgehend von unterschiedlichen sprachtheoretischen Positionen unterscheidet er zwischen einer "dimension expressive“ und einer „dimension stratégique de l'usage de la langue“, die beide einseitig und unzureichend sind und in einem antinomischen Verhältnis zueinander stehen (Judet de la Combe/Wismann 2004, S. 25 ff.). Im Bildungssystem herrsche, verstärkt durch die gesellschaftlichen und politischen Entwicklungen seit der Jahrtausendwende, eine Tendenz hin zu einer rationalistischen und utilitaristischen Sprachkonzeption mit Akzent auf dem normativen Charakter, was zu einer „Aufwertung“ der Sprache, aber gleichzeitig zu einer „ernsthaften Verringerung der Rolle von Literatur im Unterricht" führe (vgl. Judet de la Combe 2007, S. 23). Dies habe zur Folge, dass „Sprache als Funktionssprache und Servicesprache 
vorherrscht" und führe damit insgesamt zu einer „Verkümmerung der Idee von Sprache, wie man sie in der Schule unterrichtet“ (ebd., S. 34). ${ }^{7}$ Judet de la Combe versucht nun aufzuzeigen, inwiefern vor allem literarische Texte, ,in denen sich die beiden Dimensionen der Rede realisieren, einer der privilegierten Orte des Spracherwerbs und darüber hinaus der verschiedenen Verhältnisse zur Welt, welche die Sprache erlaubt, sein können“ (ebd., S. 43), denn sie verlangten eine doppelte Anstrengung:

Erstens eine positive Entfremdung, die die Konfrontation mit dem, was man nicht versteht, erzwingt [...]; zweitens die methodische und dezentrierte Wiederaneignung, nach der die Interpretation verlangt. Sie verschafft dadurch Zugang zu Sprachressourcen, die sich dem gegenwärtigen Gebrauch entziehen und neu mobilisiert werden können, um eine mögliche Zukunft zu formulieren. (ebd., S. 47)

Judet de la Combe bezieht sich hier vor allem auf die Auseinandersetzung mit altsprachlichen Texten, für die dies sicher in besonderem Maße gilt, doch grundsätzlich dürfte dies für alle Texte zutreffen, die über solche Sprachressourcen verfügen.

\subsection{Lesen und Schreiben als Lebenskunst (Wilhelm Schmid, Michel Foucault)}

Ein ganz anderer Theoriekontext ist die sogenannte „Philosophie der Lebenskunst" von Wilhelm Schmid. Inspiriert von den Arbeiten Michel Foucaults und der Auffassung, dass sich das Subjekt vor allem durch und in Sprache selbst kon-

7 Judet de la Combe führt freilich keinen harten empirischen Beleg für diese Behauptung an. Es stellt sich hier die grundsätzliche Frage, inwieweit dies empirisch untersucht werden kann. Auf jeden Fall bräuchte eine solche Untersuchung einen Denkrahmen, innerhalb dessen die entsprechenden Fragestellungen generiert und auch die Ergebnisse gedeutet würden - womit die Gefahr bestünde, dass man findet, was man sucht. Diese Grundsatzfrage kann hier nicht eingehender diskutiert werden. Nur so viel: Ich bin der Auffassung, dass jede Empirie in der Didaktik einen Denkrahmen hat und braucht und dass jeder Denkrahmen sich immer auch auf Erfahrungen stützt und damit empirisch und kein rein theoretisches Konstrukt ist. Der Grad an Explizitheit des Denkrahmens und an methodischer Kontrolliertheit der Erfahrungen hängt auch ab vom untersuchten Gegenstand. Hubert Ivo verweist in diesem Kontext mit einem Zitat aus der Nikomachischen Ethik auf die Grenzen möglicher Genauigkeit: „Denn es kennzeichnet den Gebildeten (pepaideuménu), in jedem einzelnen Gebiet nur soviel Präzision zu verlangen, wie es die Natur des Gegenstandes zulässt“ (zit. nach Ivo 2001, S. 169). Ungebildet wäre es demnach, bestimmte Formen des Empiriezwangs in erster Linie als Machtform zur Ausgrenzung und Komplexitätsreduktion zu benutzen. 
stituiert, betont Schmid die wichtige Rolle, die das Lesen und Schreiben für eine Praxis der Lebenskunst in der Gegenwart einnehmen können. So wird das Lesen selbst

zur Lebenskunst, zur Kunst des Selbst, sich selbst herzustellen und zu gestalten. Vom Text, den es berührt und von dem es berührt wird, lässt das Selbst sich subjektivieren. Es glaubt eine Geschichte zu lesen und findet und erfindet dabei in Wahrheit sich selbst. In ihm lebt die Geschichte auf, die erzählte und die eigene, die es auf dem Umweg über die Erzählung selbst zu erzählen beginnt, die Geschichte des Lebens, die sich in der Lektüre zu fügen vermag. Die Schrift regiert und dirigiert ihren Leser, indem sie ihn mit der verführerischen Klarheit der Buchstaben durch den unabsehbar weiten, weißen Raum der Möglichkeiten führt (Schmid 2012, S. 684).

Ähnlich wie bei Bieri und Nida-Rümelin wird auch hier die Bedeutung des Erzählens für die Konstruktion des Selbst und seiner Geschichte deutlich und ähnlich wie für Trabant ist das Schreiben wie auch das Lesen von Literatur für Schmid potenziell

eine Praxis der Freiheit, sowohl im Sinne einer Befreiung von Gegebenem als auch einer Formgebung der Freiheit: Gleichsam beiläufig gewinnt das Selbst im Laufe der Lektüre Möglichkeiten zu seiner Gestaltung und nutzt sie unmerklich: Die Buchstaben und Zeichen sagen, was das Selbst längst schon sagen wollte, und sagen zugleich noch etwas anderes - etwas, das das Selbst in Berührung mit dem Anderen bringt, sei die Erfahrung enttäuschend oder verheißungsvoll (Schmid 2012, S. 687).

Auf diese Weise wird das Selbst beeindruckt, geprägt und geschrieben von dem, was es liest und schreibt. Lesen und Schreiben als Lebenskunst - als Kunst, sein Leben zu deuten, zu erzählen und auch mögliche Zukünfte zu formulieren - ist das Bildungspotenzial von Literatur, das in den Bildungsstandards in ihrer gegenwärtigen Konstruktion praktisch nicht vorkommen kann und das gerade deshalb von der Didaktik umso stärker vertreten werden muss (vgl. Steinbrenner 2014b).

\subsection{Literarische Erfahrung als Resonanzerfahrung (Hartmut Rosa)}

In allen bislang vorgestellten Theorieansätzen klangen schon, mehr oder minder stark, Formen der Gesellschaftskritik an. Diese stehen im Zentrum der beiden nun abschließend vorgestellten, dezidiert gesellschaftskritischen Ansätze. Hartmut Rosa (2012) versucht in Weltbeziehungen im Zeitalter der Beschleunigung. Umrisse einer neuen Gesellschaftskritik eine Art Weiterführung der kritischen Theorie. Seine Kernthese lautet, dass es aufgrund einer vor allem auch technisch und ökonomisch bedingten Beschleunigung auf unterschiedlichen Ebenen (schnellere Ortswechsel, schnellerer Informationsfluss, weltweite Vernetzung usw.) zuneh- 
mend zu Entfremdungserfahrungen der Menschen in ihren Beziehungen zur Umwelt kommt. Diesen Entfremdungserfahrungen setzt Rosa das Konzept der „Resonanzerfahrungen“ entgegen, die seiner Ansicht nach entscheidend für ein gelingendes Leben und für Lebensqualität sind. Rosa definiert Resonanzerfahrungen als Erfahrungen

in denen die Welt den handelnden Subjekten als ein antwortendes, atmendes, tragendes, in manchen Momenten sogar wohlwollendes, entgegenkommendes oder ,gütiges' ,Resonanzsystem' erscheint. Anerkennung ist dabei ohne Zweifel eine (wichtige) Ermöglichungsbedingung von Resonanz, wenngleich Anerkennung und Resonanz nicht identisch sind: Wenn Person A von Person B wertgeschätzt oder sogar geliebt wird, bedeutet das keineswegs, dass sich zwischen $A$ und $B$ eine Resonanzbeziehung einstellt. Diese entsteht erst und nur da, wo A und B sich ,berühren', wo sie in eine Beziehung des wechselseitigen Antwortens eintreten (Rosa 2012, S. 9 f.).

Entscheidend für meinen Zusammenhang ist, dass Subjekte Resonanzerfahrungen auch außerhalb der Sphäre sozialer Interaktion machen können. So haben sich in der Moderne „die Sphären der Ästhetik und der Naturerfahrung als Resonanzsphären sui generis etabliert“ (ebd., S. 10). Das Besondere an der Resonanzerfahrung in diesen Sphären ist, dass sie sich ,nicht manipulativ sicherstellen bzw. fixieren lässt - dasselbe Musikstück, die identische Szenerie, das gleiche Ritual, welches ein Subjekt an einem Tag bis ins Innerste ergreift, kann es am nächsten Tag völlig ,kalt lassen'“ (ebd.). Genau dies stellt das Potenzial der Resonanzerfahrung dar und zwar als einer Form der Subjekt-Welt-Beziehung, die über determinierte und rein instrumentelle, kausal geprägte Relationen hinausgeht. Besonders anschaulich wird dies, wenn Rosa selbst Gedichte nutzt, um unterschiedliche Weisen des In-die-Welt-gestellt-Seins (wie „Getragenheit“ oder „Geworfenheit") zu beschreiben. Er ist der Auffassung,

dass uns die Lyrik und auch die Musik deshalb so tief berühren können, weil sie es vermögen, die Weisen des In-die-Welt-gestellt-Seins für den Rezipienten nachvollziehbar zu modellieren, zu moderieren und zum Ausdruck zu bringen (Rosa 2012, S. 385).

Die Nähe der Lyrik zur Musik ist hier wichtig, und den Begriff der Resonanz kann man durchaus wörtlich nehmen: Beim Lesen eines Gedichts kann etwas anklingen, uns ansprechen und berühren und uns eine (neue) Sprache geben für unser Erleben - genau das ist Erfahrung. Resonanzerfahrungen mit und durch literarische Texte sind zudem in den meisten Fällen "langsamere“ und nachhaltige Formen sprachlicher Erfahrung, die umso wichtiger werden in dem von Rosa beschriebenen „Zeitalter der Beschleunigung“. 


\subsection{Poesie und Deautomatisierung der Sprache (Franco ,Bifo' Berardi)}

Franco ,Bifo' Berardi ist wesentlich gesellschaftskritischer als Rosa ausgerichtet, was schon der Titel seiner Monographie Der Aufstand. Über Poesie und Finanzwesen zeigt. Hier vertritt er die provokante, aber mit vielen Beispielen belegte These, dass die Herrschaft des Finanzwesens mit ihrer Tendenz, alles in Zahlen zu erfassen und mess- und vergleichbar zu machen, auch zu einer „Automatisierung der Sprache" führt:

In jedem Bereich des menschlichen Handelns steht die Grammatik für die Errichtung von Grenzen, die einen Kommunikationsraum bestimmen. Heute ist die Ökonomie jene universale Grammatik, die die verschiedenen Stufen des menschlichen Handelns durchzieht. Die Sprache wird von ihrer ökonomischen Austauschbarkeit definiert und begrenzt: Auf diese Weise wird die Sprache auf ihren Informationswert reduziert, auf eine Stufe also, auf der technolinguistische Automatismen in den gesellschaftlichen Kreislauf der Sprache eingegliedert werden. (Berardi 2015, S. 285)

Vor allem die Poesie hat das Potenzial zur „Deautomatisierung der Sprache“, denn während die gesellschaftliche Kommunikation ein stark limitierter Prozess ist, ist die Sprache grenzenlos: Ihre Potenz ist nicht auf die Grenzen des Bezeichneten beschränkt. Die Poesie ist das Übermaß der Sprache. Sie ist der Signifikant, der aus den Grenzen des Bezeichneten befreit worden ist (ebd.).

Auch hier zeigt sich eine große Nähe zur „Freiheit des poetischen Sprechens“ bei Trabant und zur Dichtung als „Ort der Entfaltung der funktionellen Vollkommenheit der Sprache" bei Coseriu. Berardi spitzt dieses Sprachdenken politisch zu. Für ihn kann Poesie so zu einer Form des Aufstands gegen die Sprachmacht des Finanzwesens werden.

\subsection{Renaissance von Humboldts Bildungsbegriff in der Pädagogik}

Ich könnte an dieser Stelle noch auf zahlreiche weitere Ansätze vor allem aus den Erziehungswissenschaften verweisen. Der Begriff der Bildung scheint hier in der letzten Zeit eine Renaissance zu erleben, und zwar nicht nur als Sprachhülse, sonders als gehaltvolle Leitidee (vgl. u. a. das Themenheft 4/2015 der Zeitschrift für Pädagogik). Dabei wird in den meisten Fällen auf die Arbeiten Humboldts Bezug genommen und Wolfgang Sander hält fest, dass sich der ganze Diskurs um Bildung „zumindest im deutschsprachigen Raum als Fußnoten zu Wilhelm von Humboldt lesen lässt“" (Sander 2015, S. 517). Die Renaissance scheint sich aber nicht auf den deutschsprachigen Raum zu beschränken, auch im englischsprachigen Raum stößt das originär deutsche Konzept „Bildung“ auf wachsendes 
Interesse (vgl. ebd., S. 521). In vielen Fällen wird Humboldt dabei noch mit einem zu engen Fokus auf die Bildungstheorie rezipiert und der für ihn konstitutive $\mathrm{Zu}$ sammenhang von Bildungstheorie, Sprachtheorie und Anthropologie zu wenig gesehen. Genau hier kann man jedoch Kriterien für die Auswahl von Gegenständen finden, die sich für Bildungsprozesse eignen - eine Frage, die wieder zunehmend an Gewicht gewinnt. Ein Gegenstand, der hier mit Sicherheit in den Blick gerät, ist die poetische Sprache.

\section{Kontextuierung mit Ergebnissen der Studie Literaturunterricht aus Sicht der Lehrenden (Dorothee Wieser)}

Eine Studie, die versucht, Konzepte von Literaturunterricht zu erheben, die Lehrende haben (in diesem Fall Berliner ReferendarInnen), passt auf den ersten Blick nicht so recht zum Thema dieses Aufsatzes. Ich möchte jedoch der Frage nachgehen, wie sich die von Dorothee Wieser erhobenen und rekonstruierten Konzepte zu dem von mir entworfenen Denkrahmen verhalten, wenngleich die beiden Konstrukte auf sehr unterschiedlichen Ebenen angesiedelt sind. Ich werde an dieser Stelle nur die Ergebnisse der fallvergleichenden Interviewanalyse referieren und kann auf die übrige Studie wie auch deren Methodik nicht näher eingehen. Die Ergebnisse beruhen auf 15 Fällen, d.h. 15 ReferendarInnen, mit denen zu drei unterschiedlichen Zeitpunkten des Referendariats Einzelinterviews geführt wurden. Dorothee Wieser versucht die Konzepte wie folgt zusammenzufassen:

Für einen Teil der Befragten steht [...] die ,Vermittlung des Freizeitwerts des Lesens' im Vordergrund. [...] Spannung und Unterhaltung werden dabei als die zentralen Gratifikationen des Leseprozesses herausgestellt. Mit der Konzentration auf die Vermittlung des Freizeitwerts des Lesens ist aber auch ein bestimmter Blick auf Literatur assoziiert: im Vordergrund stehen die Themen bzw. Inhalte der Texte. Für die anderen Befragten ist hingegen die Zielsetzung, dass die Schüler ,sich mit Literatur auseinandersetzen' sollen, zentral. Dabei wird der Literaturbegriff normativ gebraucht, indem hochliterarische Texte als Kern des Literaturunterrichts angesehen werden, und es wird auch von den Schülern eine (gewisse) Anstrengungsbereitschaft für die Beschäftigung mit Literatur erwartet. Zudem wird der analytische Umgang mit Literatur als unabdingbarer Bestandteil des Literaturunterrichts angesehen [...]. (Wieser 2008, S. 227)

In einem Fall spricht Wieser vom Dachkonzept „Leseförderung“, im anderen Fall vom Dachkonzept „Literarische Bildung“ (ebd., S. 229). Bezeichnend ist, dass fast alle Lehrpersonen mit der Zielstufe Sek I dem Dachkonzept „Leseförderung“, alle Lehrpersonen mit der Zielstufe Sek I + II dagegen dem Dachkonzept „Literarische 
Bildung“ zugeordnet werden können (ebd., S. 231). Das Konzept von „Literarischer Bildung“ ist dabei in vielen Fällen „problematisch“, da „ein eher passives Verständnis der Vermittlung literarischer Tradition dominiert“ (ebd., S. 271), so

steht für die Mehrzahl der befragten Referendare hierbei ein abrufbares Wissen im Sinne der Bildungsdefinition von Schwanitz im Vordergrund. Eine aktive und individuelle Auseinandersetzung mit den Gegenständen der literarischen Tradition im Zuge einer historischen Selbstaufklärung, welche der Beschreibung des kulturellen Gedächtnisses bei Aleida Assmann entspricht, wird hingegen nur von wenigen in den Blick genommen (Wieser 2008, S. 270).

Gegen ein einseitiges und problematisches Verständnis literarischer Bildung führt Wieser somit die Theorie des kulturellen Gedächtnisses ins Feld. Eine sicher sinnvolle Erweiterung, wobei sich die Frage stellt, ob dies ausreicht, da Literatur nur eines von vielen Medien ist, die an der Bildung und Tradierung des kulturellen Gedächtnisses mitwirken und historische Selbstaufklärung als Ziel auch wieder etwas eng und primär mit dem Geschichtsunterricht als Fach verbunden erscheint.

Die problematische Polarisierung Leseförderung versus literarische Bildung und ein zu enges Verständnis von literarischer Bildung finden sich laut Wieser auch im literaturdidaktischen Diskurs. So hält sie nach einem Durchgang durch unterschiedliche literaturdidaktische Positionen fest, „dass bei allen Vermittlungsbemühungen Leseförderung und literarische Bildung kontrastiv gedacht werden und dass literarische Bildung zumeist an die Vermittlung kanonischer Literatur gebunden wird“ (Wieser 2008, S. 244). Der Verweis auf das „kulturelle Gedächtnis" kann diese problematische Polarisierung m. E. nicht auflösen. Vielleicht - so meine These - hängt die Polarisierung auch mit dem zu engen und einseitigen Verständnis von literarischer Bildung (und auch von Leseförderung) zusammen und kann - so meine zweite These - durch ein Konzept sprachlich-literarischer Bildung ein Stück weit aufgehoben werden.

Des Weiteren stellt Wieser in ihrem Schlussfazit fest: „Die Antinomien des Literaturunterrichts sind jedoch ein Aspekt, der in der deutschdidaktischen Lehrerbildung wohl immer noch zu wenig bzw. zu undifferenziert thematisiert wird. Allzu oft kommt es zu einseitigen Auflösungen, wie die Polarisierung von ,Leseförderung' und ,literarischer Bildung' zeigt" (Wieser 2008, S. 271). Vielleicht können die Antinomien des Literaturunterrichts eher thematisiert und bearbeitet werden, wenn sie nicht nur als Antinomien des Lehrerhandelns in der Institution Schule (vgl. u. a. Helsper 2016), sondern auch als (gegenstandsbedingte) Antinomien der Sprache gedacht werden (vgl. Kapitel 1). 


\section{Neun Aspekte sprachlich-literarischer Bildung}

Den hier entworfenen Denkrahmen möchte ich mit einem Fokus auf Literaturdidaktik und Literaturunterricht als ein „Konzept sprachlich-literarischer Bildung“ bezeichnen und in diesem letzten Kapitel in neun Aspekten zusammenfassen, was vor allem als Anregung zum Weiterdenken dienen soll.

(1) Die „Freiheit des poetischen Sprechens“ (Trabant) als grundlegende Perspektive

Die „Freiheit des poetischen Sprechens“ (Trabant) ist Ausgangspunkt und grundlegende Perspektive für die didaktische Modellierung literarischer Texte. Nimmt man die hier vertretene Perspektive an, dass sich Literatur durch die Aufhebung sprachlicher Beschränkungen auszeichnet, muss literarisches Lernen darauf gerichtet sein, Lernende zu befähigen, genau dies wahrnehmen, genießen und für sich selbst produktiv nutzen zu können (vgl. Pompe 2015, S. 217). Dies steht in Spannung zum institutionellen Kontext Schule und zum didaktischen Brauchtum, entspricht aber eher der Erwerbs- und der Gegenstandsperspektive.

(2) Sprach- und Literaturdidaktik gemeinsam denken

In einem solchen Konzept sind Sprach- und Literaturdidaktik nicht mehr voneinander getrennt, wie es in den letzten Jahren immer stärker der Fall war, sondern können ein Stück weit gemeinsam gedacht werden. Eine Literaturdidaktik, die Literatur nicht von ihrer spezifischen Sprachlichkeit her denkt, droht von der Lesedidaktik auf der einen und der Mediendidaktik auf der anderen Seite marginalisiert zu werden. Eine sich aus diesem Punkt ergebende, anschließende Fragestellung lautet, ob und wie Sprachdidaktik und Sprachunterricht bezogen auf diesen Denkrahmen weitergedacht werden können.

(3) Die Antinomien des Literaturunterrichts auch als Antinomien der Sprache denken

Im Rahmen des hier entworfenen Denkrahmens können die Antinomien des Literaturunterrichts auch als Antinomien der Sprache gedacht und dadurch vielleicht besser verstanden und bearbeitet werden.

(4) Stärkere Betonung der Mündlichkeit und der Klangdimension, der Körperlichkeit und der Sinnlichkeit von Literatur

Literatur und noch genauer gesagt das poetische Sprechen hat seinen Ursprung in der Mündlichkeit, der Körperlichkeit und der Sinnlichkeit. Dies ist auch das Fundament literarischen Lernens in allen Schulstufen, das nicht verloren und 
überlagert werden darf. In diesem Sinn spielt Literatur auch eine zentrale Rolle für den Erwerb von Literalität - und dies nicht nur im Kindergarten- und Primarschulalter, sondern lebenslang (vgl. hierzu die Ausgabe der Online-Zeitschrift Leseforum 1/2016 mit dem Titel Mündliche Literalität).

(5) Höherer Stellenwert der eigenen Produktion von Literatur

Die „Freiheit des poetischen Sprechens“ kann nicht nur durch Rezeption, sondern vor allem in der Produktion, dem Schreiben, Sprechen und Spielen von Literatur erfahren werden. Dabei sind weniger das Produkt und mehr der Prozess und die Performanz entscheidend für das literarische Lernen.

(6) Fokus auf literarische Texte, bei denen die „Freiheit des poetischen Sprechens" besonders erfahrbar wird

Dies sind u. a. Lyrik als häufig besonders „,verdichtete“ literarische Form, Sprachspiele, Poetry Slam, viele experimentelle und unkonventionelle Formen von Literatur, aber auch das autobiographische Erzählen und theatrale/dramatische Formen von Literatur. Bei diesen Formen lassen sich häufig auch Rezeption und Produktion gut miteinander verbinden.

(7) Nachdenken über Sprache und Literatur im Unterricht; stärkere Thematisierung sprachphilosophischer und -theoretischer Aspekte

Dieses Konzept und die hier entworfene Sichtweise auf Literatur sind keine „Geheimnisse“, die nur die Lehrperson kennt - im Gegenteil: Sie können und müssen mit den SchülerInnen in einer ihnen angemessenen Form thematisiert und erfahrbar gemacht werden, wie überhaupt sprachphilosophische und sprachtheoretische Aspekte stärker zum Gegenstand des Nachdenkens im Unterricht werden sollten.

(8) Geltung für alle Schulstufen und -formen

Weil dieses Konzept vor allem sprachtheoretisch und anthropologisch fundiert ist, gilt es ganz grundsätzlich als Denkrahmen für alle Schulstufen und Schulformen. Es kann und darf keine spezifische Anthropologie des Hauptschülers oder der Gymnasiastin geben. Der Denkrahmen kann dann den jeweiligen Bedingungen entsprechend gefüllt werden. Vor allem auf dieser theoretischen Grundlage kann gegen eine Marginalisierung der Literatur an Haupt- und Förderschulen und gegen einseitige Konzepte literarischer Bildung in der Sekundarstufe II argumentiert werden.

(9) Sprachlich-literarische Bildung auch in der Ausbildung von Lehrpersonen

Das hier beschriebene Konzept gilt ganz grundsätzlich auch für die Ausbildung von Lehrpersonen für alle Schulstufen. Besonders die hier genannten Aspekte 4-7 
erfordern von den Lehrpersonen spezifische Kompetenzen, die an der Hochschule erworben werden müssen. Zudem handelt es sich bei dem hier vertretenen Konzept wohl nicht nur um eine spezifische Sichtweise und Perspektive auf Literatur, sondern auch um eine spezifische Haltung und Einstellung zu Literatur. Beim „Erwerb“ einer solchen Haltung spielen die Lehrperson und Prozesse der Nachahmung eine entscheidende Rolle (vgl. Kämper-van den Boogaart 2015, S. 220 f.).

Die hier formulierten neun Aspekte sind thesenförmig zugespitzt und auch etwas provokativ formuliert. Sie haben vor allem für die von Dorothee Wieser beschriebenen einseitigen Konzepte literarischer Bildung auf der Sekundarstufe II sicher auch eine gewisse Sprengkraft.

Zielsetzungen wie „Persönlichkeitsbildung“ oder „Arbeit am kulturellen Gedächtnis“ werden hier nicht explizit genannt, sind aber keineswegs hinfällig. Sie können in diesem Denkrahmen einen spezifischen, auf Literatur und Literaturunterricht bezogenen Gehalt gewinnen.

"Sprachlichkeit des Menschen als Bildungsaufgabe in der Zeit" ist schließlich zunächst ein Denkrahmen und, wie die Formulierung sagt, eine Aufgabe, bei der noch viel an Konkretisierungsarbeit geleistet werden kann. So wurde die Frage, was „Freiheit des poetischen Sprechens" in unserer Zeit heißen kann, kaum angesprochen. Sie würde vielleicht eine spannende und neue Perspektive auf die Literaturgeschichte, -gegenwart und -zukunft ermöglichen. Viel an Konkretisierungsarbeit ist aber auch schon geleistet. So sind etliche aktuelle theoretische Grundlegungen und didaktisch-methodische Ansätze m. E. gut kompatibel mit dem hier entworfenen Denkrahmen und Konzept „sprachlich-literarischer Bildung" - auf eine kleine Auswahl möchte ich abschließend kurz eingehen. Auch ein Blick zurück in die Geschichte der Deutschdidaktik wäre lohnenswert, kann aber an dieser Stelle nicht geleistet werden.

In dem von Gerhard Härle und Bernhard Rank (2008) herausgegebenen Sammelband, Sich bilden, ist nichts anders, als frei werden. 'Sprachliche und literarische Bildung als Herausforderung für den Deutschunterricht wird eine Auseinandersetzung um den Bildungsbegriff als Denkrahmen für die Deutschdidaktik geführt. Dabei werden in zahlreichen Beiträgen die Zusammenhänge von sprachlicher und literarischer Bildung thematisiert. Die Herausgeber beziehen sich hier u. a. auch auf die Bildungstheorie und die Anthropologie in der Tradition Humboldts, was schon durch das Zitat von Bettina von Arnim deutlich wird, das dem Sammelband seinen Titel gibt.

Ansgar Nünning (2008) zeigt in seinem Aufsatz Bildung durch Sprache(n) und Literatur: Zur Aktualität von Wilhelm von Humboldts Bildungstheorie die Bedeutung von Humboldts Sprach- und Bildungstheorie für die Fremdsprachendidaktik 
auf. Vor diesem Hintergrund wendet er sich ebenfalls gegen ein einseitig instrumentelles Verständnis des Fremdsprachenlernens: Das Erlernen einer fremden Sprache ist immer mit einer neuen Welt-Ansicht verbunden, wobei der Literatur ein besonderer Stellenwert zukommt.

Thomas Zabka (2015) wendet sich in seinem programmatischen Aufsatz Was ist Hochschulreife im Umgang mit Literatur gegen einseitige und unterkomplexe Zielsetzungen im Literaturunterricht vor allem der gymnasialen Oberstufe. Mit seinen Kategorien „Immersion und Reflexion“, „Vergegenwärtigung und Distanzierung“ und „Einzelnes und Allgemeines als Erkenntnisgegenstand“ beschreibt er grundlegende Spannungsfelder des Literaturunterrichts, wobei er sich u. a. auf die Studie von Christina Vischer Bruns Why Literature? The Value of Literary Reading and What it Means for Teaching bezieht. Vischer Bruns hebt die Bedeutung der Immersion für das literarische Lernen hervor und argumentiert hier vor allem mit den Arbeiten von Donald Winnicott (die Ulf Abraham für die Literaturdidaktik entdeckt hat). Winnicott und Humboldt sind auf den ersten Blick sehr unterschiedliche Bezugspunkte, weisen m. E. aber doch etliche Gemeinsamkeiten auf, die sich weiter zu erkunden lohnten.

Anja Pompe (2015) vereinigt in dem von ihr herausgegebenen Sammelband Kind und Gedicht. Wie wir lesen lernen Beiträge, die die Bedeutung der Literatur und insbesondere der Lyrik für das Lesenlernen herausstreichen. Sie versucht aufzuzeigen, „dass die frühzeitige Erfahrung, dass es eine Sprache gibt, die anders ist als die vertraute, abhängige und eingeschränkte, von unschätzbarem Wert für unser lebenslanges Lesenlernen ist“" (Pompe 2015, S. 223). Diesen unschätzbaren Wert bestimmt Georg W. Bertram in einem Aufsatz des Sammelbandes als „artikulierte Individualität“ - das ist seine Antwort auf die Frage „Was leisten Gedichte für die eigene Sprache?" (Bertram 2015).

Was die didaktisch-methodischen Ansätze angeht, so kann zunächst das von Gerhard Härle, Johannes Mayer und mir konzipierte „Heidelberger Modell des literarischen Unterrichtsgesprächs“ genannt werden (vgl. Härle 2014; Steinbrenner/ Mayer/Rank/Heizmann 2014). Vor allem die Hermeneutiken Schleiermachers und Humboldts dienen hier zur Begründung und didaktischen Modellierung des literarischen Gesprächs als Praxis gemeinsamer Sinnsuche. Hans Lösener (2015a) nutzt in Der Rhythmus und die Fallen des Zeichens. Warum der Literaturunterricht eine Anthropologie der Sprache braucht die Sprachanthropologie Henri Meschonnics als Denkrahmen und konzipiert auf dieser Grundlage ein „hörendes Lesen“, bei dem der Rhythmus und die Stimmlichkeit literarischer Sprache im Zentrum stehen. José F. A. Oliver (2013) zeigt in Lyrisches Schreiben im Unterricht. Vom Wort in die Verdichtung zahlreiche praktische Beispiele auf, bei denen „die Freiheit des 
poetischen Sprechens" vor allem beim Schreiben erfahren werden kann. Alle drei hier genannten Ansätze sind schulstufen- und schulformübergreifend, machen sich für die Bedeutung des Literaturunterrichts gerade für „bildungsferne“ Kinder und Jugendliche stark und legen einen Fokus auch auf die Lehrerausbildung.

Cornelia Rosebrock schließlich benutzt für den Titel eines Aufsatzes, in dem sie „über Sinn und Unsinn des didaktischen Denkens in Kompetenzen“ nachdenkt (Rosebrock 2013), zwei Verse von Friedrich Hölderlin, die ich gerne hier an den Schluss stelle, wobei es mir nicht um ein Zurück zur Romantik und ihrer Literatur, Sprach- und Bildungstheorie geht, sondern um deren Wiedergewinnung und Re-Lektüre in unserer Zeit.

Voll Verdienst, doch dichterisch, wohnet der Mensch auf dieser Erde.

(Friedrich Hölderlin, In lieblicher Bläue)

\section{Literatur}

Baum, Michael (2015): Der Tanz des Bären. Zur intransparenten Transparenz von Kaspar Spinners „Elf Aspekten des literarischen Lernens“. In: Leseräume. Jg. 2015. H. 2, S. 16-27.

Becker-Mrotzek, Michael et al. (Hrsg.) (2013): Sprache im Fach. Sprachlichkeit und fachliches Lernen. Münster: Waxmann.

Berardi, Franco >Bifo (2015): Der Aufstand. Über Poesie und Finanzwesen. Berlin: Matthes \& Seitz.

Bertram, Georg W. (2015): Artikulierte Individualität. Was leisten Gedichte für die eigene Sprache? In: Pompe, Anja (Hrsg.): Kind und Gedicht. Wie wir lesen lernen. Freiburg i.Br. u. a.: Rombach, S. 33-43.

Bieri, Peter (2008): „Wie wäre es, gebildet zu sein?“. In: Göppel, Rolf et al. (Hrsg.): Bildung ist mehr. Potentiale über PISA hinaus. [9. Aufl.]. Heidelberg: Mattes, S. 13-21.

Bieri, Peter (2013): Wie wollen wir leben? München: dtv.

Coseriu, Eugenio (1994): Sprache und Dichtung. In: Albrecht, Jörn (Hrsg. und bearb.): Sprache und Dichtung: Textlinguistik. Eine Einführung. [3., überarb. und erw. Aufl.]. Tübingen/Basel: Francke, S. 146-149.

Feilke, Helmuth (2013): Bildungssprache und Schulsprache am Beispiel literalargumentativer Kompetenzen. In: Becker-Mrotzek, Michael et al. (Hrsg.): Sprache im Fach. Sprachlichkeit und fachliches Lernen. Münster u. a.: Waxmann, S. 113-130.

Frederking, Volker et al. (2013): Literarästhetische Kommunikation im Deutschunterricht. In: Becker-Mrotzek, Michael et al. (Hrsg.): Sprache 
im Fach. Sprachlichkeit und fachliches Lernen. Münster u. a.: Waxmann, S. 131-146.

Groeben, Norbert/Hurrelmann, Bettina (Hrsg.) (2004a): Lesesozialisation in der Mediengesellschaft. Ein Forschungsüberblick. Weinheim/München: Juventa.

Groeben, Norbert/Hurrelmann, Bettina (2004b): Fazit: Lesen als Schlüsselqualifikation? In: dies. (Hrsg.): Lesesozialisation in der Mediengesellschaft. Ein Forschungsüberblick. Weinheim/München: Juventa., S. 440-465.

Groeben, Norbert (2004): Einleitung: Funktionen des Lesens - Normen der Gesellschaft. In: ders./Hurrelmann, Bettina (Hrsg.): Lesesozialisation in der Mediengesellschaft. Ein Forschungsüberblick. Weinheim/München: Juventa., S. 11-35.

Härle, Gerhard/Rank, Bernhard (Hrsg.) (2008): „Sich bilden, ist nichts anders, als frei werden." Sprachliche und literarische Bildung als Herausforderung für den Deutschunterricht. Baltmannsweiler: Schneider Verlag Hohengehren.

Härle, Gerhard (2014): „... und am Schluss weiß ich trotzdem nicht, was der Text sagt“. Grundlagen, Zielperspektiven und Methoden des Literarischen Unterrichtsgesprächs. In: Steinbrenner, Marcus et al. (Hrsg.): „Seit ein Gespräch wir sind und hören voneinander". Das Heidelberger Modell des Literarischen Unterrichtsgesprächs in Theorie und Praxis. [2., korr. und erg. Aufl.]. Baltmannsweiler: Schneider Verlag Hohengehren, S. 29-65.

Helsper, Werner (2016): Antinomien und Paradoxien im professionellen Handeln. In: Dick, Michael et al. (Hrsg.): Handbuch Professionsentwicklung. Bad Heilbrunn: Klinkhardt, S. 50-62.

Humboldt, Wilhelm von (1998[1830-35]): Über die Verschiedenheit des menschlichen Sprachbaues und ihren Einfluß auf die geistige Entwicklung des Menschengeschlechts. Hrsg. von Donatella Di Cesare. Paderborn u. a.: Schöningh.

Hurrelmann, Bettina (2004): Bildungsnormen als Sozialisationsinstanz. In: Groeben, Norbert/dies. (Hrsg.): Lesesozialisation in der Mediengesellschaft: Ein Forschungsüberblick. Weinheim/München: Juventa, S. 280-305.

Ivo, Hubert (1994): Muttersprache, Identität, Nation. Opladen: Westdeutscher Verlag. Ivo, Hubert (1999): Deutschdidaktik. Die Sprachlichkeit des Menschen als Bildungsaufgabe in der Zeit. Baltmannsweiler: Schneider Verlag Hohengehren.

Ivo, Hubert (2000): Disputatio: Vermittlung von Inhalten versus Vermittlung von Kompetenzen. In: Witte, Hansjörg et al. (Hrsg.): Deutschunterricht zwischen Kompetenzerwerb und Persönlichkeitsbildung. Baltmannsweiler: Schneider Verlag Hohengehren, S. 333-340.

Ivo, Hubert (2001): Normierung und Allegorese. Deutschdidaktik als wissenschaftliche Disziplin. In: Rosebrock, Cornelia/Fix, Martin (Hrsg.): Tumulte. Deutschdidaktik zwischen den Stühlen. Baltmannsweiler: Schneider Verlag Hohengehren, S. 158-170. 
Jostes, Brigitte (2004): Die Sprachenpolitik des Europarats: Nähe und Distanz in der europäischen Mehrsprachigkeit. In: Grenzgänge. Beiträge zu einer modernen Romanistik. Jg. 11. H. 22, S. 6-30.

Judet de la Combe, Pierre (2007): Warum sich mit Sprache beschäftigen? Vorüberlegungen zu einer europäischen Bildung. Hildesheim u. a.: Olms.

Judet de la Combe, Pierre/Wismann, Heinz (2004): L'avenir des langues. Repenser les Humanités. Paris: Les Éditions du Cerf.

Kämper-van den Boogaart, Michael (2015): Literaturdidaktik und Praxeologie. In: IASL. Jg. 40. H. 1, S. 207-221.

Leseforum (2016): Mündliche Literalität. Ausgabe 1/2016. http://www.leseforum. ch. Abgerufen am 15.03.2016.

Leseräume (2015): Elf Aspekte des literarischen Lernens auf dem Prüfstand. Ausgabe 2/2015. http://leseräume.de/?page_id=308. Abgerufen am 15.03.2016.

Lösener, Hans (2015a): Der Rhythmus und die Fallen des Zeichens. Warum der Literaturunterricht eine Anthropologie der Sprache braucht. In: Interval(le)s: Réinventer le rythme / Den Rhythmus neu denken. Jg. 2015. H. 7, S. 1-22.

Lösener, Hans (2015b): Die Präzisierung der Subjektivität beim literarischen Lernen. In: Leseräume. Jg. 2015. H. 2, S. 72-84.

Lösener, Hans/Siebauer, Ulrike (2011): hochform@lyrik. Konzepte und Ideen für einen erfahrungsorientierten Lyrikunterricht. Regensburg: vulpes.

Nida-Rümelin, Julian (2013): Philosophie einer humanen Bildung. Hamburg: Körber-Stiftung.

Nünning, Ansgar (2008): Bildung durch Sprache(n) und Literatur: Zur Aktualität von Wilhelm von Humboldts Bildungstheorie. In: Burwitz-Melzer, Eva et al. (Hrsg.): Sprachen lernen - Menschen bilden. Baltmannsweiler: Schneider Verlag Hohengehren, S. 33-54.

Oliver, José F. A. (2013): Lyrisches Schreiben im Unterricht. Vom Wort in die Verdichtung. Seelze: Kallmeyer.

Ossner, Jakob (2001): Elemente eines Denkstils für didaktische Entscheidungen. In: Rosebrock, Cornelia/Fix, Martin (Hrsg.): Tumulte. Deutschdidaktik zwischen den Stühlen. Baltmannsweiler: Schneider Verlag Hohengehren, S. 17-32.

Pompe, Anja (2015): „Das hier ist Wasser“. Oder was heißt: Literarisches Lesenlernen? In: dies. (Hrsg.): Kind und Gedicht. Wie wir lesen lernen. Freiburg i.Br. u. a.: Rombach, S. 213-226.

Rosa, Hartmut (2012): Weltbeziehungen im Zeitalter der Beschleunigung. Umrisse einer neuen Gesellschaftskritik. Frankfurt a. M.: Suhrkamp.

Rosebrock, Cornelia (2013): Voll Verdienst, doch dichterisch wohnet / Der Mensch. Über Sinn und Unsinn des didaktischen Denkens in Kompetenzen. 
In: Rieckmann, Carola/Gahn, Jessica (Hrsg.): Poesie verstehen - Literatur unterrichten. Baltmannsweiler: Schneider Verlag Hohengehren, S. 25-49.

Rosebrock, Cornelia/Nix, Daniel (2014): Grundlagen der Lesedidaktik und der systematischen schulischen Leseförderung. [7., überarb. und erw. Aufl.]. Baltmannsweiler: Schneider Verlag Hohengehren.

Sander, Wolfgang (2015): Was heißt „Renaissance der Bildung“? Ein Kommentar. In: Zeitschrift für Pädagogik. Jg. 61. H. 4, S. 517-526.

Schmid, Wilhelm (2012): Mit sich selbst befreundet sein. Von der Lebenskunst im Umgang mit sich selbst. Frankfurt a. M.: Suhrkamp [ebook-Ausgabe].

Spinner, Kaspar H. (2006): Literarisches Lernen. In: Praxis Deutsch. Jg. 33, H. 200, S. 6-16.

Steinbrenner, Marcus (2007): Freiheit und Bindung. Sprachlich-literarische Bildung und die Suche nach einem Denkrahmen für die Deutschdidaktik. In: Gölitzer, Susanne/Roth, Jürgen (Hrsg.): Wirklichkeitssinn und Allegorese. Festschrift für Hubert Ivo zum achtzigsten Geburtstag. Münster: Monsenstein und Vannerdat, S. 390-420.

Steinbrenner, Marcus (2014a): „... und wenn die zwei sich in die Augen geschaut haben, haben sie noch LICHT gesehen". Literarische Erfahrungen in einem Gespräch mit einer 9. Realschulklasse. In: ders. et al. (Hrsg.) Seit ein Gespräch wir sind und hören voneinander". Das Heidelberger Modell des Literarischen Unterrichtsgesprächs in Theorie und Praxis. [2., korr. und erg. Aufl.]. Baltmannsweiler: Schneider Verlag Hohengehren, S. 263-285.

Steinbrenner, Marcus (2014b): Bildungsstandards und „die Sprachlichkeit des Menschen als Bildungsaufgabe in der Zeit" (Hubert Ivo). In: Didaktik Deutsch. Jg. 19. H. 37, S. 13-16.

Steinbrenner, Marcus/Mayer, Johannes/Rank, Bernhard/Heizmann, Felix (Hrsg.) (2014): „Seit ein Gespräch wir sind und hören voneinander“. Das Heidelberger Modell des Literarischen Unterrichtsgesprächs in Theorie und Praxis. [2., korr. und erg. Aufl.]. Baltmannsweiler: Schneider Verlag Hohengehren.

Trabant, Jürgen (2008): Was ist Sprache? München: Beck.

Trabant, Jürgen (2009): Die Sprache. München: Beck.

Trabant, Jürgen (2012): Weltansichten: Wilhelm von Humboldts Sprachprojekt. München: Beck.

Vischer Bruns, Christina (2011): Why Literature? The Value of Literary Reading and What it Means for Teaching. New York: Continuum.

Wieser, Dorothee (2008): Literaturunterricht aus Sicht der Lehrenden. Eine qualitative Interviewstudie. Wiesbaden: VS Verlag für Sozialwissenschaften.

Zabka, Thomas (2015): Was ist Hochschulreife im Umgang mit Literatur? In: Didaktik Deutsch. Jg. 20. H. 38, S. 136-150. 
Dorothee Wieser

\title{
Zum Verhältnis von Rekonstruktion, Konstruktion und Normfragen in der Deutschdidaktik
}

\section{On the relationship between reconstruction, construction and normative issues in German didactics}

\begin{abstract}
The following paper addresses the interplay between construction, reconstruction and normative issues - cornerstones of German didactic fields of action. It aims to demonstrate the necessity of taking all three dimensions into consideration at all times albeit with differing degrees of focus in each case.
\end{abstract}

\section{Selbstverständigungsfragen in der Endlosschleife?}

Dass zentrale Fragen zum disziplinären Status und zur Ausrichtung der Fachdidaktik Deutsch nach wie vor ungeklärt sind, wird immer wieder in verschiedenen Kontexten angemahnt und war wohl auch ein Anlass für die Tagung Denkrahmen der Deutschdidaktik. Dabei ist zu beobachten, dass die diesbezüglichen Debatten gewissen Konjunkturen unterliegen. Einer der letzten Konjunkturauslöser war die in Didaktik Deutsch veröffentlichte Friedrich-Preis-Rede von Werner Wintersteiner (2011). Eine kontinuierliche Auseinandersetzung mit den zentralen Streitfragen scheint hingegen nicht stattzufinden.

Doch welche Fragen sind es eigentlich, die das Debatten-Rad in Schwung halten bzw. in gewissen Abständen anstoßen? Zu nennen ist hier zunächst die Frage nach der Verortung im Wissenschaftssystem oder auch ganz grundsätzlich die Frage nach dem wissenschaftlichen Status der Disziplin angesichts der Reflexionsprobleme des Erziehungssystems (vgl. Kämper-van den Boogaart 2014; Luhmann/Schorr 1988). Eng verbunden mit diesen Fragen ist die Abgrenzung von anderen Disziplinen. Hier stand insbesondere in der Gründungsphase das Verhältnis zur Germanistik im Vordergrund (vgl. z. B. Ivo 1977, S. 7-3; Brüggemann 2014), während aktuell eher die Verortung innerhalb der empirischen Bildungsforschung dringlich und strittig erscheint. Weiteres Potential für Debatten birgt das Verhältnis von Theorie und Praxis, wobei es hier vorrangig um die Konzeptualisierung der Fachdidaktik im Rahmen der LehrerInnenausbildung 
geht, zugleich aber die Vorstellungen, die mit den Begriffen „Theorie“ und „Praxis" jeweils verbunden werden, erhebliche Unterschiede aufweisen. In der bereits erwähnten Debatte um Werner Wintersteiners (2011) Rede „Alte Meister - Über die Paradoxien literarischer Bildung" ging es hingegen vorrangig um Nähe und Distanz zum bildungspolitischen System. Gerade diese Debatte zeigt aber, dass die skizzierten Problemzonen so eng miteinander verwoben sind, dass zumeist alle berührt werden.

Die Auseinandersetzung mit diesen Selbstverständigungsfragen kann als durchaus zwiespältig empfunden werden. Einerseits ist die Beschäftigung, insbesondere mit den inzwischen schon fast historischen Positionen wie der von Hubert Ivo, äußerst anregend, weil sie die Reflexion all der Fragen einfordert, die man in Forschung und Lehre stets als irgendwie geklärt voraussetzt und dabei zugleich um die Fragilität dieser Handlungsbasis weiß. Andererseits kommt man schnell zu der Einsicht, dass es eigentlich nichts Neues zu sagen gäbe. Thomas Zabka schließt seine Besprechung des von Cornelia Rosebrock und Martin Fix (2001) herausgegebenen Bandes Tumulte. Deutschdidaktik zwischen den Stühlen mit folgenden Worten (vgl. auch den Beitrag von Juliane Köster in diesem Band):

Die besprochene Debatte ist weder von paradigmatischer Umorientierung noch von diskursiver Unordnung gekennzeichnet. [...] Bestimmte Dinge muss man immer wieder sagen. Becketts Hamm hat mit seiner Liebe zu den guten alten Gesprächen also nicht ganz Unrecht (Zabka 2002, o. S.).

Abgesehen von der Frage, wie der intertextuelle Bezug auf Becketts „Endspiel“ zu deuten wäre, ist also zu diskutieren, ob das Zirkuläre der Debatten letztlich konstitutiv für den deutschdidaktischen Diskurs ist und ausgewählte Grundsatzfragen eben in bestimmten Abständen der Disziplin immer wieder zur Reflexion vorgelegt werden müssen oder ob doch einige der Fragen einer kontinuierlichen Auseinandersetzung und Bearbeitung bedürfen.

Danach soll im vorliegenden Beitrag gefragt werden. In diesem Zusammenhang wird der Versuch unternommen, das Spannungs- und Handlungsfeld, in dem sich die Deutschdidaktik bewegt, durch die Reflexion der Dimensionen Konstruktion, Rekonstruktion und Normfragen genauer zu fassen. Es kann und soll dabei keineswegs um eine Neu-Positionierung der Fachdidaktik Deutsch gehen, sondern lediglich um die Sichtung bekannter Zusammenhänge, die aufgrund der fortschreitenden Ausdifferenzierung der Disziplin aber ab und an in den Hintergrund zu treten scheinen.

Um den Zusammenhang von Rekonstruktion, Konstruktion und Normfragen und zugleich die Spezifik fachdidaktischer Forschung in den Blick zu bekommen, soll auf John Deweys Unterscheidung zwischen einem psychologischen und ei- 
nem logischen Standpunkt zurückgegriffen werden. Diese Unterscheidung führt Dewey im Kontext seiner Überlegungen zur Konzeption des Curriculums ein:

We must take into account the distinction between a study as a logical whole and the same study considered as a psychological whole. From the logical standpoint, the study is the body or system of facts which are regarded as valid, and which are held together by certain internal principles of relation and explanation. [...] From the psychological standpoint, we are concerned with the study as a mode or form of living individual experience. (Dewey 1897; 1972, S. 168)

Diese Unterscheidung gewinnt dadurch an Bedeutung, dass sie die Differenz zwischen der Perspektive des Forschenden und der Perspektive des Lehrenden markiert und zugleich didaktische Forschungsperspektiven aufzeigt:

For the scientist, the subject-matter represents simply a given body of truth to be employed in locating new problems, instituting new researches, and carrying them through a verified outcome. [...] As a teacher he is not concerned with adding new facts to the science he teaches; in propounding new hypotheses or in verifying them. He is concerned with the subject-matter of the science as representing a given stage and phase of the development of experience. His problem is that of inducing a vital and personal experiencing. Hence, what concerns him, as teacher, is the ways in which that subject may become a part of experience; what there is in the child's present that is usable with reference to it; how such elements are to be used; how its own knowledge of subject-matter may assist in interpreting the child's needs and doings, [...]. (Dewey 1902; 1976, S. 285 f.; Herv. i. Original)

Auch wenn Deweys Sicht auf die Wissenschaften aus heutiger Sicht positivistisch anmutet, ist seine Unterscheidung der logischen und psychologischen Perspektive immer noch aufschlussreich. Dies zeigt sich unter anderem darin, dass die Überlegungen Deweys, nachdem sie im 20. Jahrhundert in der Pädagogischen Psychologie weitgehend in Vergessenheit gerieten und zunächst durch behavioristische Ansätze verdrängt wurden (vgl. Shulman/Quinlan 1996), nun beispielsweise im Rahmen von Conceptual-Change-Ansätzen ein Revival erleben. Die notwendig doppelte Perspektivierung der Gegenstände ${ }^{1}$ kann als ein zentrales Moment fach-

1 „The serious problem of instruction in any branch is to acquire the habit of viewing in a twofold way the subject-matter which is to be taught day by day. It needs to be viewed as a development out of the present habits and experiences of emotion, thought, and action; it needs to be viewed also as a development into the most orderly intellectual system possible. These two sides, which I venture to term the psychological and the logical, are limits of a continuous movement rather than opposite forces or even independent elements." (Dewey 1903; 1977, 227 f.) Auch hier könnte man natürlich kritisch anmerken, dass "the most orderly intellectual system possible“ eine jeweils zu diskutierende Setzung ist (vgl. Deng/Luke 2008, S. 73). 
didaktischer Überlegungen und Entscheidungen beschrieben werden. Zugleich zeigen die Erfahrungen in der Lehre, dass den Studierenden dieser Perspektivwechsel schwer fällt. Dies steht vermutlich zum einen mit der Tatsache im $\mathrm{Zu}$ sammenhang, dass die Studierenden gerade dabei sind, sich eine wissenschaftliche Perspektive auf die Gegenstände anzueignen und sich im wissenschaftlichen Diskurs zu orientieren und folglich eine Distanzierung vom eben Erreichten nicht naheliegend ist. Zum anderen mag es auch dadurch bedingt sein, dass für viele Bereiche insbesondere des Literaturunterrichts einzuräumen ist, dass es nach wie vor nur wenige empirische Befunde für die Entfaltung der psychologischen Perspektive, d.h. zu den relevanten Verstehens- und Entwicklungsprozessen im Umgang mit literarischen Texten, gibt. Damit kommt die Rekonstruktion von Lernprozessen als eine wohl unstrittige Zieldimension fachdidaktischer Forschung in den Blick.

Für Dewey ist die Rekonstruktion der psychologischen Perspektive untrennbar mit der Konstruktion des Curriculums verbunden. Hier zeigen sich erkennbar Differenzen zwischen der Ausrichtung der amerikanischen Curriculum Studies und der europäischen Tradition der Didaktik bzw. der sich im 20. Jahrhundert etablierenden Fachdidaktik, welche insbesondere aus den unterschiedlichen institutionellen Voraussetzungen resultieren (vgl. Hopmann/Riquarts 1995; vgl. auch Tenorth 2012, S. 13). ${ }^{2}$ In welchem Verhältnis die aktuelle Deutschdidaktik zu curricularen Überlegungen oder gar Entscheidungen steht, ist eine Frage mit hohem Debatten-Potential, auf die noch zurückzukommen sein wird.

Zongyi Deng und Allan Duke machen zudem auf eine grundlegende normative (Vor-)Entscheidung aufmerksam, die der Dewey'schen Unterscheidung inhärent ist: „However, these are not without teleological and practical limitations. Why must disciplinary knowledge be taken as the endpoint of curriculum and instruction?" (Deng/Luke 2008, S. 73). Dass auch aktuell immer noch die Notwendigkeit besteht, immer wieder darauf zu verweisen, dass Fachdidaktik keine Vermittlungswissenschaft ist, d.h. nicht auf die Erschließung des Bildungswerts und der Vermittlungsvoraussetzungen fachwissenschaftlicher Inhalte festgelegt werden sollte (vgl. Ivo 1977, S. 37 f.), das hat kürzlich Thomas Zabka in der Auseinandersetzung mit der Frage Was ist Hochschulreife im Umgang mit Literatur? betont (Zabka 2015). Da folglich Unterrichtsziele nicht aus dem Wissenschafts-

2 Markante Unterschiede sind zum einen die höchst unterschiedliche curriculare Steuerung (tendenziell lokal, d.h. in der Verantwortung der Lehrenden in den USA, eher zentral in Deutschland), zum anderen das Fehlen von Institutionen, die sich explizit der LehrerInnenausbildung widmen, wie beispielsweise die LehrerInnen-Seminare in Deutschland (vgl. Hopmann/Riquarts 1995, S. 13-17). 
system übernommen oder abgeleitet werden können, so Zabka, sind die entsprechenden normativen Größen außerhalb dessen zu suchen und zu bestimmen (vgl. ebd., S. 136). In seinem Fazit folgert er:

Zumindest in Bezug auf ästhetische Lerngegenstände brauchen wir im Fach Deutsch einen kontinuierlich geführten Dialog zwischen Schule, Wissenschaft und involvierter Öffentlichkeit - einen Dialog, der sowohl den normativen Grundlagen als auch den praktischen Anwendungen von Bildungsstandards gilt. (ebd., S. 149; Herv. i. Original)

In welchem Umfang und in welchen Formen die Auseinandersetzung über Normfragen erfolgen sollte, dürfte in der Disziplin allerdings nicht ohne weiteres konsensfähig sein. Das Verhältnis von Konstruktion, Rekonstruktion und Normfragen scheint deshalb klärungsbedürftig. Ich will im Folgenden erläutern, welche Aspekte des deutschdidaktischen Handlungsfeldes ich mit den drei Begriffen verknüpft sehe und welche Wechselwirkungen zu bedenken sind.

Abb. 1: Handlungsfelder der Deutschdidaktik

Rekonstruktion

Konstruktion

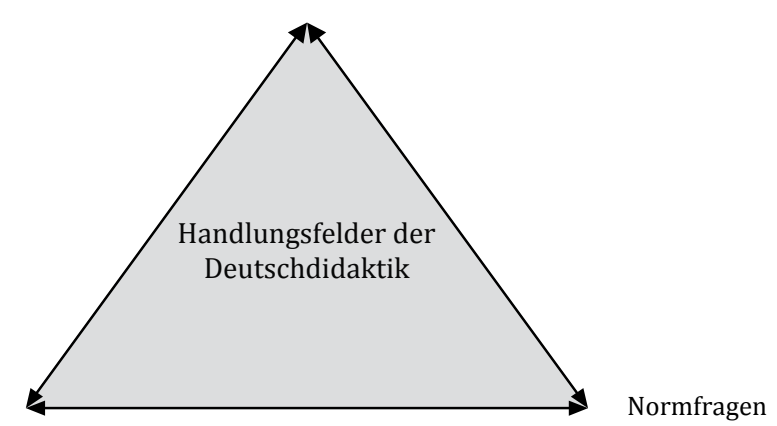

\section{Konstruktion}

Beginnen möchte ich mit dem Begriff, durch den die Spannungen zwischen dem Selbstverständnis der Fachdidaktik Deutsch und den Erwartungen von außen an die Disziplin deutlich zu Tage treten. Mit dem Begriff „Konstruktion“ sind all die Aspekte verbunden, in denen die Fachdidaktik direkt oder indirekt auf Lehr- und Lernprozesse einwirkt und damit den Modus der distanzierten Beobachtung verlässt (vgl. Tenorth 2012, S. 14). Zu nennen wäre beispielsweise die fachdidaktische Beratung bei der Entwicklung von Lehrplänen, aber auch bei der Erstellung von Abitur- und Testaufgaben und natürlich die Entwicklung von Unterrichtsmodellen. In all diesen Kontexten stellt sich stets die Frage, wie weit sich 
die Deutschdidaktik in entsprechende Prozesse einbringen kann bzw. soll. Dass damit nicht nur für die Disziplin, sondern durchaus auch für den Einzelnen oder die Einzelne heikle Punkte berührt werden, sei durch Fragen wie die folgenden angedeutet: Wie viele Artikel in Praxis Deutsch auf der Publikationsliste sind mit Blick auf Bewerbungsverfahren noch vertretbar (Stichwort: „Rezeptologie“)? Wie ist die Rolle von fachdidaktischen BeraterInnen in bildungspolitischen Kontexten bestimmt?

Diesbezüglich wäre also zu diskutieren, welche Formen der Konstruktion aus Sicht der Disziplin als sinnvoll eingeschätzt, d.h. als genuine Betätigungsfelder der Deutschdidaktik gesehen werden und nicht nur als individuelle Betätigungsfelder einiger ihrer VertreterInnen. Zudem: Auch wenn man zwischen einer angewandten und einer wissenschaftlichen Fachdidaktik unterscheidet (vgl. Gerner 2014), stellt sich trotz allem die Frage der Relationierung und Verkopplung der „wissenschaftlichen“ und der „praktischen“ Praktiken. ${ }^{3}$

Dass die Entwicklung grundlegender fachdidaktischer Konzeptionen für das Fach Deutsch, wie sie beispielsweise das Bremer Kollektiv in den 1970er Jahren intendierte, eher nicht zum Aufgabenspektrum der Disziplin gezählt wird, scheint Konsens zu sein. ${ }^{4}$ Hier hat sich die Auffassung Ivos, dass „solche Konzepte, die aus der Kritik [an dem Bestehenden] hergeleitet werden, prinzipiell nur offene und vorläufige sein können" und zudem die Eigengesetzlichkeit der unterrichtlichen Praxis stets mitgedacht werden muss (Ivo 1977, S. 142, 146), durchgesetzt bzw. für eine Zurücknahme der eigenen Ansprüche gesorgt.

Ungeklärt ist aber, wie weit oder eng die Verantwortungs- und Aufgabenbereiche der Fachdidaktik gesteckt werden sollen. Ein Irrglaube ist es in jedem Fall, wenn man meint, dass durch den Verzicht auf Konstruktion und durch den

3 Vgl. hierzu die Überlegungen von Steffen Martus zu den Verhältnissen in der Literaturwissenschaft: „,Theorie ' [Wissenschaftstheorie, Literaturtheorie] und ,Praxis [beispielsweise Textanalyse oder Interpretation] verhalten sich insofern [hinsichtlich des Verhältnisses von Wissenschaftstheorie und Wissenschaftspraxis] nicht hierarchisch zueinander; und selbst als Praxis rangiert ,Theoretisieren' nicht einfach höher als andere Praktiken. Vielmehr wäre empirisch zu klären, was von wem als, Theorie akzeptiert wird; wann aus welchen Gründen, mit welchen Absichten, Funktionsund Leistungsvisionen sich ein Fach (oder eine ,Arbeitseinheit') dazu berufen fühlt ,Theorie zu betreiben oder zu fordern; wie ,Theoretisieren' mit anderen Praktiken verkoppelt wird, sich also ins Ensemble und in die Serie von Praktiken fügt." (Martus 2015, S. 188 f.).

4 Vgl. auch Michael Kämper-van den Boogaarts kritische Reflexion zu Krefts Konzept "einer durchaus politisch gemeinten Praxisrelevanz literaturdidaktischer Forschung“ (Kämper-van den Boogaart 2014, S. 99). 
Rückzug auf distanzierte Beobachtung die Reflexionsprobleme der Disziplin als Wissenschaft eingeholt werden könnten, denn die Grenze zwischen Beobachtung und Bewertung ist schmal. Diese Selbstbegrenzung wird auch von Michael Kämper-van den Boogaart in Frage gestellt:

Es ist eine Sache, dass Wissenschaft keine Lehrpläne erlässt, dass sie aber Vorschläge machen kann, wie realistische und innovative Curricula gestaltet sein können, steht indes wahrlich auf einem anderen Blatt, auch wenn dies unattraktiver sein mag als die unreglementierte Einspeisung von Innovationen durch didaktische Publizistik. (Kämper-van den Boogaart 2014, S. 109)

Die kritische Auseinandersetzung mit Bildungsstandards oder mit Lehr- und Lernmaterialien schließt nicht nur die Reflexion und Diskussion der diesen inhärenten normativen Annahmen ein (vgl. Zabka 2015, S. 149), sondern hat letztlich stets aus einer kritisch-konstruktiven, d.h. Alternativen skizzierenden Perspektive zu erfolgen. Ansonsten wären die Funktion der Kritik und vor allem die Position, aus der diese erfolgt, höchst fraglich. Schließlich ist mit Blick auf die Enthaltsamkeit in Sachen konstruktive Einwirkung der Fachdidaktik auch zu fragen, wem man ansonsten das Feld überließe.

Noch etwas anders stellt sich die Situation mit Blick auf die LehrerInnenbildung dar, denn diese ist nicht nur historisch, sondern auch aktuell der entscheidende Grund für die Existenz und die Berechtigung der Disziplin (vgl. Kämper-van den Boogaart 2014, S. 98). Auch wenn die Befunde der Professionalisierungsforschung eine direkte Wirkung der in den fachdidaktischen Seminaren vermittelten Wissensbestände auf die Unterrichtspraxis mehr als fraglich erscheinen lassen und Reflexion als Hochwertbegriff gegenwärtiger LehrerInnenbildung fungiert (vgl. z. B. Neuweg 2011), so ist es doch zugleich unstrittig, dass im Rahmen der LehrerInnenbildung die Deutschdidaktik nicht als distanzierte Beobachterin fungiert. Dies zeigen insbesondere auch die Erfahrungen mit fallbasierter Lehre, in denen es eben nicht gelingt (und nicht gelingen kann) eine ausschließlich rekonstruktive Perspektive auf das jeweilige Fallmaterial zu etablieren (vgl. Wieser 2015). Angesichts der Stabilisierung und (in einigen Bundesländern) auch Ausweitung der fachdidaktischen Studienanteile in den Lehramtsstudiengängen ist es erstaunlich, dass eine Verständigung über Ziele und Funktionen der Deutschdidaktik im Rahmen der LehrerInnenbildung - unter Berücksichtigung der Professionsforschung - kaum stattfindet. ${ }^{5}$ Dies ist natürlich auch dadurch bedingt, dass sich

5 Ansätze zu dieser weiter zu führenden Debatte finden sich in Heft 2/2012 der Mitteilungen des Deutschen Germanistenverbandes. 
die Fachdidaktik gerade als forschende Disziplin zu beweisen sucht und Fragen der Lehre damit in den Hintergrund treten.

Spätestens im Kontext der LehrerInnenbildung muss sich die Fachdidaktik aber als konstruierende Disziplin verstehen und dies reflektieren, auch wenn die Rekonstruktion von Lern- und Lehrprozessen im Rahmen empirischer Forschung als zentrales Handlungsfeld erachtet wird. Zudem sind Konstruktion und Rekonstruktion in doppelter Weise verbunden: Auch wenn die Ableitungen für die Unterrichtspraxis aus empirischen Forschungsprojekten in ihrer Reichweite stark variieren, so lässt sich doch kaum eine Studie finden, die solche nicht zumindest implizit vornimmt. Aber auch schon die Entscheidung für eine Forschungsfrage und die Anlage der Untersuchungs-Designs dürfte, auch im Bereich der qualitativ-rekonstruktiven Unterrichtsforschung, durch Überlegungen zur Unterrichtspraxis (und deren Veränderbarkeit) bestimmt sein.

\section{Rekonstruktion}

Die Grundlagen für ihr wie auch immer eingegrenztes Handlungsfeld der konstruierenden Operationen bezieht die Fachdidaktik aus der Rekonstruktion von Lern- und Lehrprozessen. ${ }^{6}$ Die Forderung nach mehr empirischer Forschung in der Deutschdidaktik ist nicht nur ein Resultat der bildungspolitischen Zwänge nach PISA, sondern vor allem des empfundenen Spannungsverhältnisses zwischen den deutschdidaktischen Konstruktionen und deren empirischer Fundierung.

Dabei sind zwei Fragerichtungen zu erkennen. Hubert Ivo leitet aus der Annahme, dass unterrichtliche Handlungskompetenz auf der Tradierung unterrichtlichen Brauchtums basiere und zudem die Gegenstandskonstitution in unterrichtspraktischer Arbeit erfolge, Folgendes ab: „[...] dann stellt sich für die Fachunterrichtswissenschaft die erste und grundlegende Aufgabe: die Alltagswelt, in der sich diese Tradierung und Konstituierung vollzieht, zu beschreiben und nach Möglichkeit zu erklären“ (Ivo 1977, S. 183 f.). Diese Aufgabenbeschreibung Ivos lässt sich ohne weiteres an die Überlegungen von Christoph Bräuer und Torsten Pflugmacher (in diesem Band) zur Ausrichtung deutschdidaktischer Forschung mit dem Ziel der Rekonstruktion von Unterrichtspraktiken und der sie konstituierenden Normen und Strukturen anschließen.

Die zweite Forschungsrichtung ließe sich im Sinne Deweys mit „Fundierung des psychologischen Standpunkts" beschreiben. Es geht folglich um die Unter-

6 Rekonstruktion wird hier bewusst weit gefasst und nicht auf die qualitativ-rekonstruktive Forschung begrenzt. Zudem ist die Rekonstruktion von Schreib- und Leseverstehensprozessen mit eingeschlossen. 
suchung von Lernprozessen und der sie beeinflussenden sozialen und psychologischen Faktoren. Das Spektrum reicht dabei von Studien außerhalb des unterrichtlichen Kontextes, die als eine Form der Grundlagenforschung verstanden werden können, bis hin zu Interventionsstudien, welche die konkrete Wirksamkeit bestimmter Verfahren überprüfen. Dabei sind die Differenzen zwischen fachdidaktischen und kognitionspsychologischen Untersuchungen beispielsweise des Text-, Metaphern- oder Symbolverstehens aufschlussreich: Zum einen gilt es auch bei den Studien, die außerhalb des unterrichtlichen Kontextes durchgeführt werden, die Anschlussfähigkeit an die unterrichtlichen Lern- und Lehrprozesse nicht aus dem Blick zu verlieren. Dabei erweist es sich häufig als schwierig, eine Balance zwischen (externer) Validität auf der einen und Objektivität und Reliabilität auf der anderen Seite herzustellen. Die Schwierigkeiten, die beispielsweise Ursula Christmann und Norbert Groeben aus kognitionspsychologischer Perspektive für die Untersuchung des Metaphernverstehens beschreiben (vgl. Christmann/Groeben 2013), haben für die fachdidaktische Forschung besondere Relevanz, wenn die Forschungsergebnisse nicht nur den Gütekriterien der Forschung genügen, sondern auch im fachdidaktischen Diskurs und gegebenenfalls darüber hinaus Bedeutung erlangen sollen. Dass in kognitionspsychologischen Studien nicht mit unkonventionellen Metaphern gearbeitet und zudem die Kontextuierung vernachlässigt wird - die aber gerade aus literaturdidaktischer Perspektive von besonderem Interesse wären - erklären Christmann und Groeben wie folgt:

Man steht hier also vor einem grundlegendem Dilemma: Eine hochgradige experimentelle Kontrolle erfordert die Verwendung eher konventionellen Sprachmaterials - zugleich macht die Verwendung kreativen Sprachmaterials den Verzicht auf hochauflösende Methoden unvermeidbar! Es ist dies das (in der Psychologie) sattsam bekannte Dilemma zwischen sog. quantitativen und qualitativen Methoden, zwischen interner (experimenteller) und externer (Alltags-)Validität als Skylla und Charybdis der empirischen Forschung. (ebd., S. 150)

Für fachdidaktische Forschungsvorhaben ist dieser Befund eine permanente Herausforderung. Vor allem aber ist es in fachdidaktischen Untersuchungssettings nur bedingt möglich, die untersuchten Schreib-, Lese- und Verstehensprozesse rein deskriptiv darzustellen. In einem gewissen Umfang geht es stets auch um die Bewertung derselben und damit um die Frage der normativen Implikationen der Bewertungskriterien. Wie bereits angedeutet, ist zudem schon die Auswahl bzw. Fokussierung bestimmter Aspekte in der Untersuchung von Verstehensprozessen oder die Konzeption eines Interviewleitfadens interessengeleitet und von bestimmten Annahmen zum Deutschunterricht beeinflusst. 
Die Frage, was eine deutschdidaktische Studie von einer linguistischen, soziologischen, kognitionspsychologischen Untersuchung unterscheidet, wäre weiter zu verfolgen. Sehr wahrscheinlich wäre das Antwortspektrum bei den VertreterInnen der Disziplin sehr groß. Während einige die Differenzen betonen, werden sie von anderen eher marginalisiert. Auch hier spielt natürlich die jeweilige (erwünschte) Verortung im Wissenschaftssystem eine zentrale Rolle. Diskrepanzen lassen sich aber auch bezüglich der Einschätzung der Relevanz und Priorisierung von Forschungsfragen ausmachen. Bei Hubert Ivo (1977) und Jakob Ossner (2001; siehe auch den Beitrag im vorliegenden Band) ist dabei eine sehr parallele Argumentation hinsichtlich der Verbindung von Rekonstruktion, d.h. Lehr-Lernforschung, und Konstruktion, d.h. Ableitungen für die Unterrichtspraxis erkennbar. Beide gehen von der Komplexität des LehrerInnenhandelns aus und leiten daraus die Notwendigkeit von empirisch fundierten Entscheidungshilfen ab: Empirische Befunde sollen das LehrerInnenhandeln beispielsweise im Rahmen der Fehlerdiagnostik entlasten (Ivo 1977, S. 55-110) oder zu einer realistischen Einschätzung von Erfolgswahrscheinlichkeiten bestimmter Methoden führen (Ossner 2001, S. 27).

Diese Sicht auf die Verbindung von Rekonstruktion und Konstruktion ist aber keineswegs eine geteilte. Es wird auch der Rückzug bzw. die Konzentration auf die Position der Rekonstruktion, auf die Beschreibung unterrichtlicher Praktiken, gefordert. So schreibt Christian Dawidowski im Rahmen der bereits erwähnten Debatte zur Rede von Werner Wintersteiner:

Eine Literaturdidaktik, die sich „gerne als reine Handlungswissenschaft sieht“ (Wintersteiner 2011, 11), erliegt somit nicht nur der Gefahr, mit der Literatur das eine Objekt ihrer Reflexion aus dem Blick zu verlieren, sondern opfert mit der nur noch praxisfixierten Reflexion hochkomplexer Vermittlungsprozesse auch einen Gutteil ihrer Glaubwürdigkeit als empirisch abgesicherte Reflexionswissenschaft. Damit verschiebt sich das interdisziplinäre Gefüge, in dem die Literaturdidaktik an der Literaturwissenschaft, der Pädagogik und dem schulisch-bildungspolitischen Zusammenhang partizipiert, in bedenklichem Maße - der Preis ist mit der (zumindest partiellen) Einbuße von Wissenschaftlichkeit zu hoch. (Dawidowski 2012, S. 9)

Somit ist also erneut der fragile disziplinäre Status angesprochen, der eine bestimmte Ausrichtung der Forschungsfragen nahelegt. Es sind aber nicht nur die unterschiedlichen Verortungen und Anbindungen im Wissenschafts- oder Erziehungssystem, die für Differenzen sorgen. Eng verknüpft damit sind auch unterschiedliche Vorstellungen von den Aufgabenfeldern und -funktionen der Disziplin. 


\section{Normfragen}

Dass alle Operationen auf dem Feld der Konstruktion mit normativen Setzungen verbunden sind, ist unstrittig. Aber ebenso sind alle empirisch-rekonstruktiven Zugänge in einem normativen Horizont verortet, der allerdings häufig unzureichend reflektiert wird. Wenn Luhmann mit Blick auf Entwicklungen der kritischen Soziologie der 1970er Jahre schreibt, dass die „kritische Pose [...] die Darstellung eines eigenen Interesses" erspart bzw. dieses negiert (Luhmann 1993, S. 248), trifft dies meines Erachtens auch auf die Positionierung der Deutschdidaktik als kritische Wissenschaft in Abgrenzung von einer Handlungswissenschaft zu. ${ }^{7}$

So zentral die kritische Reflexion der normativen Setzungen in bildungspolitischen Vorgaben oder die Offenlegung der impliziten Normen der unterrichtlichen Praxis im Rahmen der rekonstruktiven Forschung sind: Die Normen und Konventionen des deutschdidaktisch Forschenden dürfen gleichfalls nicht ausgeblendet werden. ${ }^{8}$ Diese zu reflektieren und zu benennen, ist im Kontext einer reflexiven Sozialforschung unabdingbar und zugleich eine nicht unbeträchtliche Herausforderung und nur inter-individuell, d.h. durch Beobachtung der Beobachtung zu leisten. Zudem ist zu fragen, inwiefern sich aufgrund der Involvierung des bzw. der Forschenden in Prozesse des Erziehungssystems ausschließlich uneinholbare Reflexionsprobleme oder auch Potentiale für die Deutschdidaktik zeigen. Auch hier scheint mir eine Beobachtung Luhmanns von Interesse: „Anscheinend können Funktionssysteme gerade dann, wenn sie ,Dogmatik' als Grundlage ihrer Selbstbeschreibung akzeptieren, hinter diesem Schutzschirm größere Reflexionsfreiheiten mobilisieren“ (ebd., S. 256). Hier kommt man also wieder zu der Frage zurück, inwiefern eine Selbstbeschreibung der Deutschdidaktik als kritisch-reflexive Wissenschaft tragfähig ist bzw. überhaupt als nützlich und nicht vielmehr als hinderlich erscheint.

Schon die Beschreibung und Erklärung unterrichtlichen Brauchtums erfolgt also stets vor einem normativen Horizont. Erst recht werden normative Setzun-

7 Vgl. auch die Skepsis Ivos gegenüber den Bestrebungen der Fachdidaktik in den 1970er Jahren, sich einem emanzipatorischen Erkenntnisinteresse in Anlehnung an Habermas zu verschreiben (Ivo 1977, S. 133-139).

8 „Die Alltagsorientierung [am Unterricht] ermöglicht den Verzicht auf a priori geltende Vorgaben der Werte und der Erziehungsziele, man ,findet' sie im Alltag der Erziehungspraxis. Aber dann hat man die Frage, wie man zu diesen Vorhaben Distanz gewinnt und mit welchen Unterscheidungen man sie übernehmen kann." (Luhmann 2002, S. 193) 
gen relevant, wenn man die Komplexität des unterrichtlichen Handelns durch wissenschaftliche Befunde reduzieren will (vgl. Ivo 1977; Ossner 2001) oder eine andere Form von Ableitungen aus den empirischen Ergebnissen fachdidaktischrekonstruktiver Forschung für die Unterrichtspraxis anstrebt.

Die Auseinandersetzung über Norm- und Zielfragen - als Grundlage der Reflexion derselben in Forschungsprojekten - ist aber zumindest gegenwärtig in den Hintergrund des fachdidaktischen Diskurses getreten bzw. verbirgt sich hinter der Kompetenzdebatte. Diese Situation erschwert die Explikation von normativen Vorannahmen im Rahmen von Forschungsprojekten. Wenn in empirischen Forschungsvorhaben der Forschungsstand aufgearbeitet wird, dann wird meist auf soziologische, kognitionspsychologische, sprach- und literaturwissenschaftliche (etc.) Studien Bezug genommen. Eine Verortung in einem normativen Koordinatenfeld der Deutschdidaktik erfolgt somit höchstens indirekt, weil dieses Koordinatenfeld im aktuellen Diskurs kaum expliziert bzw. bearbeitet wird und Forschungsberichte nicht der Ort für entsprechende Explikationen sind.

Aus den bisherigen Überlegungen zu Konstruktion, Rekonstruktion und Normfragen in der Deutschdidaktik lassen sich zwei Fragen bzw. Diskussionsräume ableiten, die für die Selbstverständigung der Disziplin von Relevanz sein könnten:

- Dies ist erstens weniger die Frage nach dem wissenschaftlichen Status, sondern zunächst die nach der Aufgaben- bzw. Funktionsbeschreibung der Fachdidaktik Deutsch.

- Unabhängig davon, wie diesbezüglich Rekonstruktion und Konstruktion gewichtet werden, erscheint zweitens eine offen geführte Auseinandersetzung über Normen und Ziele des Deutschunterrichts bedeutsam - nicht nur, aber auch um durch die Akzeptanz der Dogmatik größere Reflexionsfreiheiten zu gewinnen.

Man kann sich in dem von Konstruktion, Rekonstruktion und Normfragen abgesteckten Feld unterschiedlich positionieren. Zieht man sich auf eine Position zurück, verlässt man das fachdidaktische Spiel. Entscheidend ist vielmehr, die Fäden straff zu halten, d.h. für den steten reflektierten Bezug der drei Positionen zu sorgen. Einen Punkt, der aus meiner Sicht derzeit für eine gewisse Schlaffheit der Fäden sorgt, möchte ich nun nochmals aufgreifen, wobei auch dieser keineswegs ein neuer Aspekt im Diskurs ist: die Verständigung über die Ziele des Deutschunterrichts. 


\section{Formen der Verständigung über Ziele und Inhalte}

\subsection{Angst vor den Zielen?}

In seinem Beitrag in dem bereits erwähnten „Tumulte“-Band stellt Jakob Ossner fest:

Ich habe in den letzten zehn Jahren nirgends eine tiefere Diskussion über Zieldifferenzierung feststellen können. Leitend sind falsche Alternativen wie „Deutschunterricht zwischen Kompetenz- und Persönlichkeitsbildung", wie das Motto des Germanistentages in Lüneburg hieß, - als könnte das eine Alternative sein. In der Beschreibung der Sektion des Symposions, in der wir uns hier befinden, wird die „Konkurrenz zwischen Gegenstands- und Schülerorientierung" zwar als unfruchtbar ausgewiesen, ihre Existenz aber dennoch konstatiert und nach dem „Status dieser Grenze“ gefragt. Eine tiefer gehende Zieldiskussion wird auf diese Art eher verhindert als befördert. (Ossner 2001, S. 25)

Ossner führt weiter aus, dass durch die fehlende Auseinandersetzung über Ziele, „das Pferd von hinten aufgezäumt wird. Das Inszenierungspotential für Ziele - sei es die ,Bilderfolge' als Schreibanlass und Schreibspur, sei es der handlungs- und produktionsorientierte Literaturunterricht - wird selbst Zielgenerator" (ebd., S. 26). Es wäre zu prüfen, inwiefern diese Feststellung Ossners heute auf die Kompetenzmodellierung bzw. auf den Zusammenhang von Kompetenzüberprüfung und Kompetenzmodellierung zutrifft: Viele der vorliegenden Beschreibungen von Kompetenzniveaus basieren letztlich auf der Beschreibung von Aufgabenschwierigkeiten. Wenn aus entsprechenden Kompetenzmodellierungen dann wieder Ziele abgeleitet werden, schließt sich der Kreis (vgl. Steinmetz 2014, S. 17).

Auch zehn Jahre später scheint sich die Situation nicht grundlegend gewandelt zu haben, denn Werner Wintersteiner beklagt in seiner debattenauslösenden Rede gleichfalls die Abstinenz in Sachen Zieldiskussion - wenn auch aus einer etwas anderen Perspektive:

Im deutschen Sprachraum [im Gegensatz zu Frankreich, so Wintersteiner] vermisst man diese leidenschaftliche öffentliche Debatte über den Stellenwert von literarischer Bildung ebenso, wie man die innerfachliche Diskussion über die Konsequenzen für das Selbstverständnis und die Positionierung des Faches vermisst, eine grundsätzliche, epistemologische und programmatische Debatte. (Wintersteiner 2011, S. 8)

Woran liegt es aber, dass die Diskussion über Ziele des Deutschunterrichts immer wieder eingefordert, letztlich aber nur vage geführt wird - beispielsweise um die Frage, ob der Deutschunterricht der Bildung von Humankapitel verpflichtet sei (vgl. ebd.; Kammler 2011; Zabka 2011) oder sich in unermüdlichen Auseinandersetzungen mit dem Kompetenzparadigma verliert? Es sind mehrere Gründe denk- 
bar: Einen Grund führt Thomas Zabka in seiner bereits erwähnten Rezension des Tumulte-Bandes an:

Entgegen Ossners Ansicht dürfte die verbreitete fachdidaktische Zurückhaltung in Sachen "Zielgenerierung" nicht allein in der mangelnden „Anstrengung" der Fachleute, sondern zum Teil eben doch „in der Natur der Sache“ [Verweis auf den Beitrag Ossners] begründet sein - nämlich in kognitiven, emotionalen und sozialen Prozessen der Rezeption und Produktion von Texten. (Zabka 2002, o. S.)

Ausgehend von diesem Einwand, der von den notwendig offenen, wenn auch nicht unbegrenzten Prozessen in der unterrichtlichen Verständigung über Literatur ausgeht, werden Differenzierungen notwendig: Zum einen müssten unterschiedliche Zieldimensionen beschrieben werden. Wenn es um die Festlegung einer bestimmten, von den SchülerInnen zu „erfassenden“ Interpretation geht, dann ist die von Thomas Zabka angemahnte Zurückhaltung unumgänglich. Anders dürfte die Einschätzung ausfallen, wenn Zielsetzungen reflektiert werden, wie sie beispielsweise in den Bildungsstandards für den Mittleren Schulabschluss oder für die Allgemeine Hochschulreife formuliert werden. ${ }^{9}$ Im Kontext entsprechender Zielbestimmungen wäre neben der Frage nach der Sinnhaftigkeit derselben zu diskutieren, in welchen Schulstufen und -formen solche Ziele als erreichbar eingeschätzt werden und welche Kriterien zur Überprüfung des Erfolgs als angemessen erscheinen (vgl. Steinmetz 2014; vgl. auch den Beitrag von Juliane Köster in diesem Band). Es zeigt sich also, dass bei der Auseinandersetzung um Ziele nicht nur die Zielrichtung, sondern auch das angestrebte Niveau bedacht werden muss. Dass dies wiederum nur auf der Basis von empirischen Untersuchungen geschehen kann, ist einsichtig.

So wichtig klare Positionierungen und daraus resultierende Debatten über Ziele des Deutschunterrichts - mit geringer, mittlerer und größerer Reichweite - erscheinen, so entscheidend ist zugleich die Rückbindung an (empirische) Studien, die sich beispielsweise der Rekonstruktion von Erwerbsprozessen, differenten Schreib- und Textverstehensstrategien oder der „Wisdom of Practice“ der Lehrenden widmen. Nur so können wechselseitige Reflexionen ermöglicht, fragliche Konventionen aufgedeckt und didaktische Wunschprosa entlarvt werden.

9 „[Die Schülerinnen und Schüler können] Mehrdeutigkeit als konstitutives Merkmal literarischer Texte nachweisen“ oder „ihr Textverständnis argumentativ durch gattungspoetologische und literaturgeschichtliche Kenntnisse über die Literaturepochen von der Aufklärung bis zur Gegenwart stützen" (Bildungsstandards im Fach Deutsch für die AHR 2012, S. 20). 
Der stete Bezug auf die Praxis des Deutschunterrichts ist aber auch deshalb relevant, weil die „Sektionierung“ bzw. „Partialisierung“ der Fachdidaktik Deutsch (vgl. den Beitrag von Juliane Köster) schon weit fortgeschritten ist. Munter wird in den verschiedenen Sektionen geforscht und diskutiert, ohne dass zumindest geprüft wird, inwiefern sich Zielkonflikte ergeben, oder die Verwobenheit der Prozesse im Unterricht bedacht wird. Das ausgewogene Zusammenspiel von Konstruktion, Rekonstruktion und Normfragen ist also vor allem über die „Sektionen“ der Deutschdidaktik hinweg zentral und muss gesichert werden.

Nicht zuletzt ist die Relevanz von Forschungsfragen (und damit verbunden ihre finanzielle Förderungswürdigkeit) nicht unabhängig von der disziplinären Diskussion um Zielsetzungen zu bewerten, will man sich nicht ausschließlich an den Prioritätensetzungen der Drittmittelgeber orientieren. Die Erdung der Zieldiskussionen und auch die Bestimmung von Zielfragen, die aktuell dringlich zu diskutieren wären, können wiederum nur durch die Rückbindung an empirische Beobachtungen und Rekonstruktionen gewährleistet werden.

Dass die Ziele des Deutschunterrichts ein weites Feld sind, braucht wohl nicht ausgeführt zu werden. Es ist also keineswegs trivial, die Punkte zu bestimmen, an denen "tiefer gehende Zieldiskussionen“ (Ossner 2001, S. 25) als dringlich erscheinen: Sind es vor allem Diskussionen um die in den Klassenstufen zu erreichenden Niveaus und um die Gewichtung von Bildungsstandards? Geht es um die Wertschätzung bestimmter Wissensbestände und -formen? Und nicht zu vergessen: Welche Relevanz hat die Auseinandersetzung über die Auswahl von Gegenständen in Zeiten der Kompetenzorientierung innerhalb der Fachdidaktik Deutsch? Liegen diese Fragen vor allem in der Verantwortung der Schulen, welche schulinterne Curricula konzipieren (vgl. Zabka 2011, S. 27)? Oder muss hier die Fachdidaktik Angebote unterbreiten; Angebote, die nicht nur Gegenstände benennen, sondern diese aus einer Erwerbsperspektive modellieren und konstruktive Fragestellungen für die Auseinandersetzung mit den Gegenständen aufzeigen (vgl. Kämper-van den Boogaart 2011, S. 36)? Die Frage nach den Zielen führt also wieder zur Aufgabenbestimmung der Fachdidaktik und insbesondere zu Fragen des Ausmaßes der Konstruktion im Rahmen der Fachdidaktik.

Aber selbst wenn man diesen Überlegungen zustimmt, bleibt doch die Frage, wo eigentlich Raum für solche Positionierungen und Debatten in der Deutschdidaktik ist. Die Redaktion von Didaktik Deutsch hat sich gerade in den letzten Jahren sehr bemüht, Diskursräume zu eröffnen. Zugleich dominieren Beiträge zu empirischen Forschungsvorhaben und die Relationierung der beiden Bereiche („Debatte“ - „Forschungsbeiträge“) stellt eher die Ausnahme dar. Der Befund Ossners aus dem Jahr 2001, wonach in Didaktik Deutsch ,im wesentlichen didak- 
tische Erörterungen allgemeiner Art bevorzugt werden“ (Ossner 2001, S. 29), trifft heute ganz offensichtlich nicht mehr zu. Nun ist ganz sicher niemand an einem Revival „didaktischer Erörterungen allgemeiner Art“ interessiert. Spezifische didaktische Erörterungen, welche empirische Befunde mit fachdidaktischen, erziehungswissenschaftlichen (modern: bildungswissenschaftlichen) und auch sprach- und literaturwissenschaftlichen Diskursen in Beziehung setzen und damit Positionen zur Diskussion stellen, sind aber ein Desiderat.

\subsection{Fazit}

Mein Fazit kann somit knapp ausfallen: Mindestens zwei Fragen sind aus meiner Sicht kontinuierlich von der Fachdidaktik Deutsch zu bearbeiten und sollten nicht nur in konjunkturbedingten Debatten aufscheinen. Diese beiden Fragen liegen allerdings auf unterschiedlichen Ebenen: Auf einer übergeordneten Ebene liegt die Frage nach den Aufgabenfeldern der Deutschdidaktik. Bei der Diskussion dieser Frage sollte die Sehnsucht nach dem verbürgten Status der Wissenschaftlichkeit zurückgestellt werden und eine offene und durchaus auch pragmatische Diskussion über die Funktionen der Deutschdidaktik geführt werden (vgl. Ivo 1977, S. 138).

Mit Blick auf das für die Fachdidaktik konstitutive Zusammenspiel von Konstruktion, Rekonstruktion und Normfragen sind es aus meiner Sicht vor allem die Norm- bzw. Zielfragen, die aktuell wieder stärker berücksichtigt werden müssten. Zugleich ist aber zu betonen, dass dies keineswegs eine Absage an die Empirie ist - im Gegenteil. Ich habe versucht darzulegen, wie die verschiedenen Zugänge einander bedingen (müssen).

Schließen möchte ich mit einem Zitat aus dem nach wie vor aktuellen Buch Zur Wissenschaftlichkeit der Didaktik der deutschen Sprache und Literatur von Hubert Ivo:

Die wissenschaftliche Bearbeitung der Fragen des Deutschunterrichts wird auf die Dauer nur erfolgversprechend sein, wenn 1. alle Fragerichtungen zugelassen sind und entsprechende Forschungsprogramme ausgearbeitet werden; 2. die mit den Fragerichtungen gesetzten einschränkenden Bedingungen gesehen, aber bewußt in Kauf genommen werden; 3. die „Synthetisierung" der je gewonnenen Ergebnisse und ihrer Aspekte als eigenes wissenschaftliches Problemfeld anerkannt und bearbeitet wird. Diese These richtet sich gegen die Konzeption von Didaktik der deutschen Sprache und Literatur, die didaktische Arbeit ausschließlich als Reflexion auf historisch-gesellschaftliche Voraussetzungen und Funktionen des Deutschunterrichts und seiner Bezugswissenschaft versteht. Eine solche Konzeption setzt die Kenntnis der empirisch antreffbaren Unterrichtswirklichkeit immer schon als gesichert voraus und verpaßt oder versperrt gar die Möglichkeit einer wissenschaftlichen Überprüfung dieser Kenntnis. Sie richtet sich ferner gegen die Konzeption, die die 
Probleme der Legitimierung von Wertentscheidungen aus der wissenschaftlichen Arbeit ausscheidet und damit deren „kritische“ Möglichkeiten erheblich einschränkt. Sie richtet sich schließlich gegen Konzeptionen, die auf letzte Gewissheiten rekurrieren und damit die prinzipielle Offenheit wissenschaftlicher Arbeit - jedenfalls in bestimmten Punkten außer Kraft setzen. (Ivo 1977, S. 54 f.; Herv. i. Original)

Damit ist eigentlich alles, in diesem Falle insbesondere von Hubert Ivo, schon gesagt worden. Es bleibt mir nur die aktualisierende Wiederholung.

\section{Literatur}

Bildungsstandards im Fach Deutsch für die Allgemeine Hochschulreife (Beschluss der KMK vom 18.10.2012) (2012): http://www.kmk.org/dokumentation/ veroeffentlichungen-beschluesse/bildung-schule/qualitaetssicherung-inschulen.html\#c7035. Abgerufen am 24.02.2015.

Brüggemann, Jörn (2014): Deutschdidaktik und Germanistik. Analyse einer umstrittenen Beziehung. In: Frederking, Volker/Krommer, Axel (Hrsg.): Taschenbuch des Deutschunterrichts. Aktuelle Fragen der Deutschdidaktik (3). Baltmannsweiler: Schneider Verlag Hohengehren, S. 143-176.

Christmann, Ursula/Groeben, Norbert (2013): Zwischen Skylla und Charybdis: Kognitionspsychologische Ansätze zur Metapher. In: Lessing, Marie/Wieser, Dorothee (Hrsg.): Zugänge zu Metaphern - Übergänge durch Metaphern. Kontrastierung aktueller disziplinärer Perspektiven. Paderborn: Fink, S. 145-160.

Dawidowski, Christian (2012): „Bildung“ oder: Fachdidaktik als Wissenschaft. Ein Zwischenruf. In: Didaktik Deutsch. 18. Jg., H. 32, S. 5-9.

Deng, Zongyi/Luke, Allan (2008): Subject Matter. Defining and Theorizing School Subjects. In: Connelly, F. Michael/He, Ming Fang/Phillion, JoAnn (Hrsg.): The SAGE handbook of curriculum and instruction. Los Angeles: Sage Publications, S. 66-87.

Dewey, John (1972): The Psychological Aspect of the School Curriculum (1897). In: Boydston, Jo Ann (Hrsg.): The Early Works of John Dewey. 1882-1898, Carbondale: Southern Illinois University Press, Ill., S. 164-176.

Dewey, John (1976): The Child and the Curriculum. In: Boydston, Jo Ann (Hrsg.): The middle works. 1899-1924. (Bd. 5, 1902-1903). Carbondale: Southern Illinois University Press, Ill., S. 271-291.

Dewey, John (1977): The Psychological and the Logical in Teaching Geometry (1903). In: Boydston, Jo Ann (Hrsg.): The Middle Works. 1899-1924. (Bd. 3, 1903-1906). Carbondale: Southern Illinois University Press, Ill., S. 216-228.

Gerner, Volker (2014): Die Didaktikwissenschaft Deutsch und ihre Bezüge zur Bildungswissenschaft/Erziehungswissenschaft/Pädagogik. In: Frederking, Volker/Krommer, Axel (Hrsg.): Taschenbuch des Deutschunterrichts. Aktuelle 
Fragen der Deutschdidaktik (3). Baltmannsweiler: Schneider Verlag Hohengehren, S. 177-196.

Hopmann, Stefan/Riquarts, Kurt (1995): Didaktik und/oder Curriculum. Grundprobleme einer international vergleichenden Didaktik. In: Hopmann, Stefan/ Riquarts, Kurt (Hrsg.): Didaktik und/oder Curriculum. Beiheft 33. Weinheim: Beltz, S. 9-34.

Ivo, Hubert (1977): Zur Wissenschaftlichkeit der Didaktik der deutschen Sprache und Literatur. Vorüberlegungen zu einer „Fachunterrichtswissenschaft. Frankfurt am Main/Berlin/München: Diesterweg.

Kammler, Clemens (2011): Kryptische Selbstkritik. Zu Werner Wintersteiners Preisrede „Alte Meister - Über die Paradoxien literarischer Bildung“ auf dem Symposion Deutschdidaktik 2010. Didaktik Deutsch. 17 (31), S. 5-10.

Kämper-van den Boogaart, Michael (2014): Fachdidaktik als Wissenschaft. In: Kämper-van den Boogaart, Michael (Hrsg.): Deutsch-Didaktik. Leitfaden für die Sekundarstufe I und II. [5. Auflage]. Berlin: Cornelsen, S. 90-111.

Kämper-van den Boogaart, Michael (2011): Zur Fachlichkeit des Literaturunterrichts. Didaktik Deutsch. 17 (30), S. 22-39.

Kreft, Jürgen (2001): Deutschdidaktik zwischen Wissenschaft und Sprache oder Literatur, Germanistik und Pädagogik: Asymmetrien und Defizite. In: Rosebrock, Cornelia/Fix, Martin (Hrsg.): Tumulte. Deutschdidaktik zwischen den Stühlen. Baltmannsweiler: Schneider Verlag Hohengehren, S. 8-16.

Luhmann, Niklas/Schorr, Karl-Eberhard (1988): Reflexionsprobleme im Erziehungssystem. Frankfurt am Main: Suhrkamp.

Luhmann, Niklas (1993): „Was ist der Fall?“ und „Was steckt dahinter?“. Die zwei Soziologien und die Gesellschaftstheorie. Zeitschrift für Soziologie. 22(4), S. 245-260.

Luhmann, Niklas (2002): Das Erziehungssystem der Gesellschaft. Hrsg. von Dieter Lenzen. Frankfurt am Main: Suhrkamp.

Martus, Steffen (2015): Wandernde Praktiken „after theory“? Praxeologische Perspektiven auf „Literatur/Wissenschaft“. IASL (Internationales Archiv für Sozialgeschichte der deutschen Literatur). 40(1), S. 177-195.

Neuweg, Georg Hans (2011): Distanz und Einlassung. Skeptische Anmerkungen zum Ideal einer „Theorie-Praxis-Integration“ in der Lehrerbildung. Erziehungswissenschaft 22(43), 2011, S. 33-45.

Ossner, Jakob (2001): Elemente eines Denkstils didaktischer Entscheidungen. In: Rosebrock, Cornelia/Fix, Martin (Hrsg.): Tumulte. Deutschdidaktik zwischen den Stühlen. Baltmannsweiler: Schneider Verlag Hohengehren, S. 17-33.

Shulman, Lee S./Quinlan, Kathleen M. (1996): The Comparative Psychology of School Subjects. In: Berliner, David C./Calfee, Robert C. (Hrsg.): Handbook 
of Educational Psychology. New York: MacMillan Library Reference USA, S. 399-422.

Steinmetz, Michael (2014): Zurück zu den Basics! Anmerkungen zu den Bildungsstandards Deutsch. Didaktik Deutsch. 19(37), S. 16-19.

Tenorth, Heinz-Elmar (2012): Forschungsfragen und Reflexionsprobleme - zur Logik fachdidaktischer Analysen. In: Bayrhuber, Horst et al. (Hrsg.): Formate fachdidaktischer Forschung. Empirische Projekte - historische Analysen theoretische Grundlegungen. Münster u. a.: Waxmann, S. 11-27.

Wieser, Dorothee (2015): Theorie(?)-Praxis-Konstellationen in Lehrerforschung und Lehrerbildung: Fragen an die aktuelle deutschdidaktische Lehrerforschung. In: Bräuer, Christoph/Wieser, Dorothee (Hrsg.): Lehrende im Blick. Empirische Lehrerforschung in der Deutschdidaktik. Wiesbaden: VS, S. 17-34.

Wintersteiner, Werner (2011): Alte Meister - Über die Paradoxien literarischer Bildung. Didaktik Deutsch. 17 (30), S. 5-21.

Zabka, Thomas (2002): Theorie des Deutschunterrichts zwischen pädagogischen und philologischen Zielsetzungen. Rezension zu: Rosebrock, Cornelia/Fix, Martin (Hrsg.): Tumulte. Deutschdidaktik zwischen den Stühlen. (Diskussionsforum Deutsch 6). http://www.iaslonline.de/index.php?vorgang_id=2508. Abgerufen am 26.02.2015.

Zabka, Thomas (2011): Wir bilden auch „Humankapital“. Gegen einige bildungskritische Vereinfachungen. Didaktik Deutsch. 17 (31), S. 22-29.

Zabka, Thomas (2015): Was ist Hochschulreife im Umgang mit Literatur? Didaktik Deutsch. 20 (38), S. 136-150. 
Christoph Bräuer - 978-3-631-70204-8

Downloaded from PubFactory at 01/11/2019 10:39:03AM

via free access 
Jakob Ossner

\title{
Didaktische Konstrukte und Konstruktvalidität in der Deutschdidaktik
}

\section{Didactic constructs and construct validity in German didactics}

\begin{abstract}
The paper examines the construct validity of didactic literacy models and explains why the task of German didactics is to provide teachers with clear constructs on which they can base didactic teaching decisions and select appropriate and expedient materials and methods.
\end{abstract}

\section{Vorbemerkung}

Grundsatzdiskussionen sind gewöhnlich Zeichen einer Krise. Daher scheinen sich solche für die Fachdidaktik nach der bildungspolitischen Wende nach 2000 erübrigt zu haben. Seitdem ist die Fachdidaktik im Aufwind, auch wenn man die Beschwörung der Wichtigkeit und Notwendigkeit der Fachdidaktik selbst als ein Krisenphänomen deuten kann. Im Ensemble der Bildungswissenschaften hat nun auch die Fachdidaktik die Aufgabe, Zahlen zu liefern, denn, so unisono die Klage: „Wir wissen zu wenig.“ Die Vorstellung einer evidenzbasierten Deutschdidaktik leuchtet am Horizont. Wie in der Medizin nur noch das verordnet, verschrieben und therapiert werden soll, wofür es Erfolgsevidenz gibt, weil große Studien den Erfolg gezeigt hätten, so soll in der Schule auch nur noch das durchgeführt werden, was die nötige Effektstärke aufweist.

Der Grundgedanke ist bestechend, die Details sind höchst komplex, wie beispielsweise Hatties Daten zu Problemlösen und problembasiertem Lernen zeigen. Während Ersteres eine Effektstärke von $\mathrm{d}=.61$ hat, hat Letzteres eine von $\mathrm{d}=.15$, also keinen Effekt (vgl. Hattie 2013). Über den Grund kann man nur spekulieren. Was die Evidenzbasierung so schwierig macht, das ist die Relativität der Daten. Genau das macht die Hattie-Studie so brisant. Es werden Daten, die aus ganz verschiedenen Forschungsgebieten stammen, die unter ganz verschiedenen Fragestellungen gewonnen wurden, von dieser ihrer Relativität befreit und nur noch auf ein Maß, das der Effektstärke, hin befragt. Nun erscheinen die Dinge absolut. Man erfährt bei Hattie nicht, ob das problembasierte Lernen nur deswegen so schlecht abgeschnitten hat, weil nur Klassen, die sich an die 7-Sprung-Methode 
gehalten haben, untersucht wurden oder auch Klassen, die den Grundgedanken aufgenommen haben, ohne sich der Langeweile des immer Gleichen auszusetzen.

Bevor die Fachdidaktik Erfolgsdaten liefert, muss sie sich um die Datengewinnung sorgen sowie um eine angemessene Interpretation der Daten. Für eine empirische Wissenschaft ist es entscheidend, dass der Denkrahmen, innerhalb dessen Daten erhoben werden sollen, ausgewiesen ist. Man schreckt erst auf, wenn die Daten nicht zu stimmen scheinen, um anzumelden, was da noch hätte bedacht werden müssen.

Hinsichtlich der jeweiligen Konstruktvalidität stehen Theorie und Empirie in einem Bedingungsverhältnis: Empirische Daten bereichern die Theorie, aber das theoretische Konstrukt bedingt das empirische Testkonstrukt. Ich erspare mir die Hinweise darauf, wie häufig Fragestellungen ausgeklammert werden müssen, weil sie in kein Testformat passen oder Daten über zweifelhafte Rating-Verfahren geglättet werden.

Vielleicht ist es wieder Zeit, nach Schulungen in empirischem Arbeiten, nachdem die Fachdidaktik gelernt hat, wie die Psychologie zu funktionieren, sich auf die eigene Aufgabe zu besinnen.

\section{Themenfelder für eine Fachdidaktik}

Als eine praktische Wissenschaft bemüht sich die Fachdidaktik um Antworten auf die Frage: „Was soll im Unterricht des Faches Deutsch getan werden?“ Lässt man den grundsätzlich ethischen Rahmen, den jede Praxis bedenken muss und in den vor allem Rollendefinitionen auf der Grundlage eines Menschenbildes eingehen, vorläufig weg, so hat man hier mit dem zu tun, was Kant einen hypothetischen Imperativ nennt. Es geht also um die Frage, welches die geeignetsten Mittel für einen vorgestellten Zweck sind.

Wenn man diesem Gedanken folgt, dann sind die unmittelbaren AnsprechpartnerInnen der Fachdidaktik die Lehrkräfte; ja, sie wären es auch noch dann, wenn die Antwort auf die Frage „Was soll getan werden?“ wäre: „Kinder lernen umso mehr, je weniger sie belehrt werden“" (Bredow/Hackenbroch 2013, 100). Denn selbst in diesem extremen Fall muss z. B. immer noch die Anlauttabelle verfertigt und verteilt werden und vor allem müssen im Verlaufe des Lernprozesses Texte von SchülerInnen gelesen und Anregungen gegeben werden.

Wenn die Lehrkräfte die AnsprechpartnerInnen sind, so sind die SchülerInnen die wesentlichen Größen, ohne die die Frage „Was soll getan werden?" nicht beantwortet werden kann. Allerdings sind die SchülerInnen nicht als abgefüllte Kompetenzpäckchen fassbar, ihre Entwicklung ohne den Kontext des Unterrichts, der diese Entwicklung initiiert und vorantreibt, nicht beschreibbar. Das Urmodell 
der Lese- und Schreibentwicklung von Frith mit einer ausgeprägten logographemischen Phase, formuliert auf der Grundlage des englischen Schulunterrichts, entpuppt sich bald als nicht so geeignet für deutsche Schulen, die in den 80er Jahren, in denen das Modell erstellt wurde, längst die synthetische Methode oder Methodenintegration praktizieren (vgl. Wimmer/Hummer 1990).

Vielleicht ist hier der wesentliche Unterschied zur Psychologie: Diese interessiert sich (u. a.) für die Lernergebnisse. Wenn in PISA Länder miteinander verglichen werden, so ist nur das Ergebnis interessant, der dieses Ergebnis hervorbringende Unterricht dagegen kaum. Er kommt erst ins Spiel, wenn man mit dem Ergebnis nicht zufrieden ist und das Ergebnis verbessern möchte. Fachdidaktik beginnt demnach nach PISA. Vielleicht wäre es aber gut, wenn Fachdidaktik vor PISA sich anstrengen würde, indem es Konstrukte liefert, die nach mehreren Dimensionen hin transparent sind. Dann wüsste man genauer, was gemessen wird.

Der Aufschrei der Literaturdidaktik gegen PISA war, dass die Art des Lesens, die PISA maß, nicht besonders interessant sei, dass gegenüber dem Konzept, Lesen als Informationsentnahme zu modellieren, die Emotionalität des Leseaktes (vgl. Abraham et al. 2003) bzw. Lesen als kulturelle Praxis (vgl. Fingerhut 2002; Hurrelmann 2002) fokussiert werden müssen. Bis heute hat aber die Literaturdidaktik kein Textmodell, das über das van Dijk und Kintsch (1983) hinauswiese, bereitgestellt. Was man aber sehen kann, ist, dass sich heute ein Literaturunterricht des Gesprächskreises gegen einen Leseunterricht mit Lesetraining absetzt. Das ist keine begrüßenswerte Entwicklung.

Ein Zweites kommt hinzu. Die häufigste empirische Untersuchungsform ist, wenn ich es recht sehe, heute die Interventionsstudie, die den Erfolg von Konzepten oder Methoden messen möchte. Dabei kommen oft sehr überraschende Befunde heraus, mitunter ganz gegensätzliche, wie man schon früh sehen kann. Ich erinnere an die Studie von Klotz (1996) zu Grammatische Wege zur Textgestaltungskompetenz, in der Klotz nachwies, dass Grammatikunterricht für etwas gut sein kann, während sich etwa zur gleichen Zeit in der Studie von Melenk (1998) zur Rechtschreibkompetenz zeigte, dass grammatisches Wissen zur Lösung von Interpunktionsproblemen nicht tauglich zu sein scheint.

In Didaktik Deutsch 35 von 2013 vergleichen Neubauer \& Kirchner zwei Modelle der Rechtschreibförderung bei DrittklässlerInnen. Eines nach Reuter-Liehr (2006; 2008), das Kinder Wörter in Silben zerlegen lässt, und eines von SchulteKörne und Mathwig (2001/2004), das nach dem Vokalquantitätsansatz arbeitet. Bei Reuter-Liehr lautet die Anweisung: „Ich schreibe den Mitlaut doppelt, wenn ich ihn beim genauen Einhalten der Silbenpause zweimal sprechen muss"; bei Schulte-Körne: „1. Enthält das Wort einen lang gesprochenen Selbstlaut? (Nein), 
2. Hörst du anschließend mehrere Mitlaute) (Nein), 3. Hörst du nur einen Mitlaut, dann verdoppele ihn." (Zit. nach Neubauer/Kirchner 2013, S. 49).

Auf den ersten Blick scheint hier alles bestens zu sein. Tatsächlich aber werden zwei Konzepte verglichen, die in einer 3. Klasse schlecht zu vergleichen sind. Reuter-Liehr modelliert die Doppelkonsonantenbuchstabenschreibung, häufig auch "Schärfungsschreibung" genannt, rein phonographisch. Schulte-Körne dagegen orthographisch, als besondere Regel des Deutschen. In der 3. Klasse eine phonographische Regel anzubieten, ist nicht unangebracht, aber ein Schritt zurück in das Anfangsstadium des Schreibenlernens. Ganz anders bei SchulteKörne, dessen Regel ganz andere Anforderungen hat, nicht zuletzt weil sie die Vokalquantität als unterscheidbar ansetzt und rein kognitiv formuliert ist.

Untersucht wird also weniger, welches der beiden Konzepte besser abschneidet, sondern ob es sinnvoll ist, in der 3. Klasse noch einmal zwei Schritte zurückzugehen und mehr oder weniger noch einmal von vorne anzufangen. In der Tat zeigen sich dann auch bei den schwächeren SchülerInnen deutliche Effekte bei Reuter-Liehr. Daher wäre eine sinnvolle Ausgangfrage gewesen, ob nicht für schwächere SchülerInnen in einem Unterricht, der die Vokalquantität und Regelwissen fokussiert, ein silbisch orientierter Unterricht günstiger wäre. Dazu aber hätten die beiden Konstrukte analysiert und dann sinnvoll gegeneinandergestellt werden müssen.

Diese Bemerkungen zielen sowohl auf das didaktische Konstrukt, hier bezogen auf die Konsonantenbuchstabenverdoppelung, als auch auf die Durchführungsbestimmungen. Im Großen geht es dabei um das Curriculum; im Kleinen um das Material für den Unterricht, für Förderung bis hin zur konkreten Stunde.

Beides verweist auf zu treffende Entscheidungen auf der Grundlage von Zielen - zuerst von Fundamentalzielen, in denen ernsthaft gesagt wird, was erreicht werden soll und auch geprüft wurde, ob es erreicht werden kann und dann von Instrumentalzielen, die geeignet sind, die Fundamentalziele zu erreichen. Bevor darauf zurückzukommen sein wird, ein weiterer Gedanke.

Bekanntlich kann das Erreichen eines Handlungsziels nicht logisch aus Umständen geschlossen werden, also in dem Sinne, dass jemand X tut und sich dann $\mathrm{Y}$ einstellt. Ob sich Wirkungen einstellen, ist in der Tat eine empirische Frage, die aber nur dann seriös beantwortet werden kann, wenn tatsächliche Wirkungsfaktoren in der Konstruktion des Modells identifizierbar sind. Um dies zu gewährleisten, muss das empirisch zu überprüfende Konstrukt so weit als nur irgend möglich transparent sein; das heißt nichts anders als dass die Entscheidungen, die zu einem Konstrukt führten, nachvollziehbar sein müssen. 
Vor welchen Problemen man dabei stehen kann, zeigt eine Betrachtung einer Untersuchung in Heft 36 von Didaktik Deutsch. Hier stellen Bangel und Müller ein Konzept zur Förderung morphologischer Bewusstheit vor, das zur Stärkung der semantischen Worterkennung konzipiert wurde und der inhaltlichen Leseförderung dienen soll (vgl. Bangel/Müller 2014). Mit dem Letzteren hat man ein hierarchisch sehr hoch angesiedeltes Instrumentalziel, ein Fundamentalziel kann es nicht sein, weil Lesen kein Wert an sich ist. Förderung dessen, was morphologische Bewusstheit genannt wird, ist auf alle Fälle ein Instrumentalziel. Die Bedeutung dieses Instrumentalziels wird nachzuweisen versucht, indem auf die Graphie der Wörter verwiesen wird, die im Deutschen von der Morphemkonstanz geprägt sei. Dazu gibt es alternative Ansätze, dass es sich nämlich nicht um eine Morphemkonstanz handele, sondern um eine Schemakonstanz auf der Grundlage phonologischer Wörter (vgl. Ossner 1996). Dieser Unterschied in der Herangehensweise ist bei der Morphemklasse der Derivationssuffixe auffällig, die nicht konsonantisch beginnt (-ig, -ung etc.) Diese Morpheme haben wiederum Auswirkungen auf Wortstämme, bei denen Schwa-Elision eine besondere Rolle spielt, beispielsweise Atmung trotz Atem, Handlung trotz handeln etc. Jetzt hat man keine einfache Morphemkonstanz mehr. Wiese (2000, S. 242 ff.) erklärt diese Phänomene über eine e-Epenthese; die Stämme sind also atm- und handl- und werden durch eine Schwa-Epenthese zu Atem und handeln. Wiese hat für das Phänomen eine phonologische Erklärung, weswegen es reizvoll wäre zu untersuchen, ob ein phonologisch ausgerichtetes Konzept weiter trägt als ein morphologisches. Und vielleicht könnte man dabei auch untersuchen, wo die Grenzen eines jedes Konzepts sind. Nimmt man beispielsweise an, dass die Silbifizierung von Wörtern auf der Grundlage phonologischer Wörter (zur Definition vgl. Yu 1992, S. 34) durchgeführt wird, so wird ein morphologischer Ansatz überflüssig (vgl. Ossner 1996, S. 390 ff.). Daraus folgen entsprechend andere didaktische Vorstellungen als bei einem Ansatz, der ein starkes morphologisches Prinzip unterstellt, insbesondere dann, wenn man dieses Prinzip unabhängig von der Phonologie denkt.

Alle diese Fragen werden in dem erwähnten Artikel überhaupt nicht diskutiert, vielmehr wird mit Verweisen auf Eisenberg, Furhop, Primus, Bredel, Müller, Hinney und mit dem Ausdruck „schriftstrukturell orientierter Sprachunterricht" (mit Verweis auf Furhop/Müller und Hinney) so getan, als gäbe es nichts mehr zu erörtern. Dafür wird als Training ein relativ schlichtes Übungsprogramm zu „Wortfamilien bilden“, „Wörterstämme unterstreichen“, „Wörter in Bausteine segmentieren“ vorgestellt. Dabei wäre "schuld\#ig“ von einigem Interesse, insbesondere dann, wenn, wie oben ausgeführt, zuvor ein Silbenkonzept des oben angedeuteten Zuschnitts unterrichtet wurde und die SchülerIinnen [ $\int \mho 1 \mid$ diç] 
als Gliederung internalisiert hätten. Vom einbettenden Unterricht erfährt man allerdings kaum etwas. Überhaupt hat man es in diesen Untersuchungen immer mit geschichtslosen SchülerInnen zu tun. Die Untersuchung setzt in der 5. Klasse an, was davor war, spielt offensichtlich keine Rolle.

Keine Rolle spielt aber offensichtlich auch, dass die Bestimmung von Morphemen seine Tücken hat; nicht nur atmen oder Handlung sind hier Beispiele, sondern selig, emsig, verlieren, Vernunft und vernünftig usw. Ob daher, um ein explizit genanntes Beispiel der Untersuchung von Bangel und Müller zu nennen, das Unterstreichen des Wortstammes \#steh\# in verstehen zur Bedeutungserkennung beim Lesen verhilft, darf man bezweifeln.

Als Erfolg werden unterschiedliche Effekte ausgewiesen (vgl. S. 54): Bei morphologischer Bewusstheit $\mathrm{d}=.71$ in der Interventionsklasse, bei der Dekodierfähigkeit allerdings nur $\mathrm{d}=.26$, also mehr oder weniger vernachlässigbar, und bei der Lesegeschwindigkeit ergibt sich sogar ein leichter, allerdings unter statistischen Gesichtspunkten vernachlässigbarer Überlegenheitseffekt bei der Kontrollklasse von $d=.13$. Dass das Erkennen von Wortstämmen und Wortbausteinen, sowie das Bilden von Wortfamilien einen Effekt haben, sollte allerdings nicht überraschen. Überraschend wäre, wenn 360 Min. Training nichts gezeitigt hätten. Allerdings hat dieses Training nichts oder nur mehr oder weniger Vernachlässigbares ,in der Lernentwicklung der Schülerinnen und Schüler [...] bezogen auf die abhängigen Variablen Dekodierfähigkeit und Lesegeschwindigkeit“ (S. 46) bewirkt. Eine Diskussion hierüber findet kaum statt. Insbesondere werden weder der Umstand, dass Ergebnisse aus dem englischen Sprachraum mit einer anderen (orthographischen) Wortstruktur nicht unmittelbar auf das Deutsche übertragen werden können, noch der, dass isoliertes grammatisches Training selten Erfolge in anderen sprachlichen Bereichen hat, diskutiert, vor allem aber wird das Untersuchungskonstrukt nicht selbst kritisch in den Blick genommen.

Was kann man an dem Beispiel lernen?

- Es gibt Denkkollektive, die gerne unter sich bleiben und sich auch gerne gegenseitig zitieren und bestätigen.

- Die Untersuchung basiert nicht auf einer sorgfältigen Konstruktvalidität. Um zu beurteilen, ob überhaupt und ggf. wie die gemessenen Effekte zu deuten sind, müsste man den Unterricht, den vergangenen und den gehaltenen, sowie das genaue Wortmaterial kennen.

Die hohe Effektstärke bei der sogenannten „morphologischen Bewusstheit“ gaukelt also etwas vor, gegenüber dem aber höchste Skepsis angebracht ist. Damit wird nicht die gemessene Effektstärke bezweifelt, sondern dass sie eine Entschei- 
dungshilfe sein kann. Es geht aber in der Didaktik darum, dass sie Entscheidungshilfen für das Unterrichten bereitstellt, und nicht darum, dass Entscheidungen über entsprechende Effektstärken abgenommen werden.

\section{Ein einfaches Entscheidungsmodell}

Im Folgenden geht es mir darum zu zeigen, dass für alle Arten von Handlungen Entscheidungen zu treffen sind. Dabei unterscheidet sich ein Testkonstrukt nicht von einem didaktischen Unterrichtskonstrukt und mein Augenmerk wird im Folgenden mehr auf Letzterem sein, weil mir die Aufgabe der Didaktik nicht darin zu bestehen scheint, dass sie viel testet, sondern valide Konstrukte für den Unterricht vorlegt, die in Entscheidungen einfließen können.

Bei Entscheidungen stellen sich letztlich immer dieselben Fragen.

1. Welche Handlungsalternativen habe ich?

2. Was sind die Ziele und welche maßgeblichen Bedingungen sind bei der Handlungsdurchführung zu bedenken?

3. Wie hoch ist der Wert einer Handlungsalternative unter einer bestimmten Bedingung einzuschätzen?

4. Wie wahrscheinlich ist, dass eine bestimmte Folge eintritt, wenn eine Alternative unter einer bestimmten Bedingung durchgeführt wird?

Wie in anderen praktischen Wissenschaften auch, sollte die universitäre Deutschdidaktik Entscheidungsstellen identifizieren und Studierende und dann Lehrkräfte befähigen, begründbare Entscheidungen zu treffen, indem sie zeigt, was im Einzelnen zu bedenken ist.

In eher seltenen Fällen wird dann die Lehrkraft alles selbst entscheiden müssen; meistens muss sie entscheiden, ob ein Angebot an Material als Grundlage für Handlungsalternativen für die jeweiligen Ziele brauchbar ist. Dabei wäre es sehr wünschenswert, dass das Material so transparent gemacht ist, dass es hinsichtlich der eigenen Ziele, der eigenen maßgeblichen Bedingungen und der vor Ort angenommenen Wahrscheinlichkeiten eingeschätzt werden kann.

In etwa kann man sich das Ganze - im Folgenden am Beispiel des Rechtschreibens, das durch das amtliche Regelwerk bestimmt ist - so vorstellen:

\section{Abb. 1: Entscheidungsparameter}
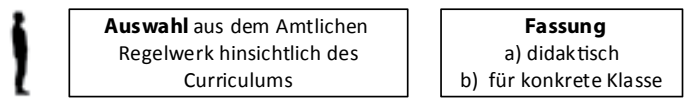

Beurteilung des vorhandenen Materials mit Blick auf die konkrete Klasse 
Um das Ganze transparent zu machen, werde ich es an einem Beispiel diskutieren, das gegenwärtig von einigem Interesse ist, der Behandlung der Doppelkonsonantenbuchstabenschreibung, die oben schon bei Neubauer und Kirchner anklang.

Bei der Auswahl des Stoffes haben Lehrkräfte die entsprechenden Vorgaben im Curriculum. Die Entscheidung einer einzelnen Lehrkraft ist hier vielleicht noch, wann im Schuljahr der Stoff behandelt wird. Vielfach wird diese Entscheidung aber heute in einem schuleigenen Curriculum von allen Lehrkräften meist für viele Jahre getroffen. Der Stoff „Konsonantenbuchstabenverdoppelung“ vielleicht wird er auch „Schärfung“ oder "Schärfungsschreibung“ genannt - ist gewöhnlich für die 2. Klasse vorgesehen.

Interessant ist die Fassung dieses basalen Laut-Buchstaben-Bereichs. Hier sind eine Reihe wesentlicher Entscheidungen zu fällen, die ich im Folgenden in ihren grundsätzlichen Strukturen darzustellen versuche.

Gegenwärtig werden verschiedene Positionen für die Fassung der Doppelkonsonantenbuchstaben in der Schreibung angeboten. Dabei steht die Didaktik vor einem typischen Problem. Sie muss nämlich meistens erst den Kern der Positionen herauspräparieren, bevor die didaktische Argumentation im eigentlichen Sinne überhaupt beginnen kann. Im Wesentlichen kann man die Positionen so fassen: Die Doppelkonsonantenbuchstabenschreibung ist ein Phänomen der Schrift, als solche gehört sie in die orthographische Phase, weil sie über eine einfache, alphabetische Zuordnung von Buchstaben zu Lauten hinausgeht. In diesem Fall ist die Buchstabengemination im Wesentlichen eine Lesehilfe.

Die Gegenposition erklärt sie dagegen phonographisch, als Ergebnis der Zerlegung des Redestroms in Silben.

Dahinter steckt ein weiteres Problem von einiger didaktischer Brisanz. Was sind die basalen Einheiten für die Graphie: Wörter, das nimmt die erste Position an, oder Silben, das nimmt die zweite Position an.

Wie kann ein/e DidaktikerIn sich in diesem außerdidaktischen Lagerstreit, der keineswegs so klar vorliegt, wie er eben geschildert wurde, entscheiden? Dies ist keine einfache Frage, da es bis heute nur wenig Tradition in der Entscheidung solcher Fragen gibt.

Das Vorgehen des Didaktikers bzw. der Didaktikerin kann nur sein, dass er die Frage vor dem Hintergrund seiner Ziele verfolgt. Es geht ja nicht um einen abstrakten Streit, sondern um die Frage, mit welcher Modellvorstellung das Rechtschreiben am besten erlernt werden kann. Dabei muss man „am besten“ dreifach ausbuchstabieren: 
- hinsichtlich der Rechtschreibung selbst: Gegenstandskonstitution;

- hinsichtlich der Entwicklung einer Person: Personenangemessenheit und

- im Rahmen der Institution: Potential für eine unterrichtliche Prozessierung im Rahmen der Institution.

Ich werde den letzten Gesichtspunkt im vorliegenden Fall vernachlässigen, da hier beide Positionen vor ähnlichen Problemen stehen, und die ersten beiden betrachten.

Hinsichtlich der Gegenstandskonstitution kann die Sprachdidaktik zuerst nur die verschiedenen Ansätze konstatieren. Welchen sie vorzieht, wird sie dann unter Anwendung des Occam'schen Rasiermessers und didaktischer Argumente zu entscheiden haben. Nach Occam ist diejenige Theorie, die mit den wenigsten Hypothesen und Termini auskommt und damit einfacher ist, zu bevorzugen. Eine in diesem Sinne einfachere Theorie muss allerdings nicht didaktisch einfach sein. Dies wird sie erst dann, wenn die gegenstandsbezogene Einfachheit nicht mit hoher Komplexität erkauft ist.

Vor diesem Hintergrund sollen nun zwei verschiedene Ansätze verglichen werden: Ein Ansatz, der auf der Drucksilbe basiert und in Form der Röber'schen sogenannten „silbenanalytischen Methode“ vorliegt, sowie ein Ansatz, der mit dem Konzept der Schallsilbe operiert. ${ }^{1}$

Die Syllabierung gemäß der Schallsilbe findet sich in ihrer klassischen Form bei Wiese (2000, S. 36):

\section{Abb. 2: Ambisyllabische Konsonanten}

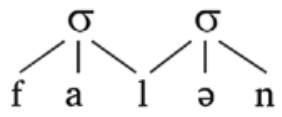

In der historischen Sprachwissenschaft hat man dabei von der Schließung kurzer offener Tonsilben gesprochen, die im Übergang zum Neuhochdeutschen flächendeckend vollzogen wurde. Ein Wort wie lëder kann den Weg nhd. [le: | dər] oder

1 Den Ansatz, den Schulte-Körne \& Mathwig wählen, und der unmittelbar auf der Fassung des $₫ 2$ des amtlichen Regelwerks beruht, werde ich nicht weiter verfolgen. Zum einen ist hinlänglich bekannt, dass Vokalquantität von den meisten SchülerInnen einer 2. Klasse nur sehr unzulänglich erfasst wird, was aber nicht gegen den Ansatz in höheren Klassen spricht. Zum andern sollen zwei Ansätze verglichen werden, die beide vor dem Hintergrund der Silbe operieren, wohingegen ein vokalquantitativer Ansatz direkt auf das Wort zielt. Es wird sich aber unten zeigen, dass der Röber'sche Ansatz ebenfalls als vokalquantitativer Ansatz gefasst werden kann. 
plattdt. [led | dər] gehen. Wird der Akzentvokal gedehnt, wird die Silbe offen, bleibt sie kurz, kommt es zu einer Ambisyllabifikation, wie in Abbildung 2 ausgedrückt. Vennemann (1986, S. 39) verweist hierbei auf das Prokosch'sche Gesetz, wonach „eine akzentuierte Silbe [...] um so stärker bevorzugt [ist], je näher ihr Gewicht bei zwei Moren liegt. (Die optimale akzentuierte Silbe ist zweimorig.)“ Eine kurze Tonsilbe (akzentuierte Silbe) kann nur durch Ambisyllabifikation zweimorig werden - oder sie wird gelängt und kann dann offen sein.

Dagegen sieht die grafische Darstellung bei Röber so aus:

Abb. 3: Sogenannter "fester Anschluss" bei Röber

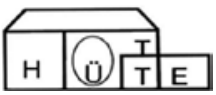

Dagegen zeigt Abb. 4 die grafische Darstellung von Hüfte, wo die beiden Silben deutlich zu sehen sind:

Abb. 4: Sog. „loser Anschluss“ bei Röber



Zugrunde liegt hier die Maas'sche Phonetik, die ein „Spiralenmodell“ annimmt.

Mit einer Spirale wird eine abgeschlossene Silbe gegliedert;

beim losen Anschluss $(\rightarrow$ ist ein entsprechender Abschluss möglich wie bei Rate:

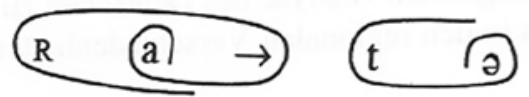

Bei festem Anschluss (ل) wird der Vokal dagegen an das Folgesegment angeschlossen. Wenn dieses nicht im Endrand der gleichen Silbe steht, sondern als Anfangsrand der Folgesilbe fungiert (die zwangsläufig eine Reduktionssilbe ist) wird diese insgesamt in die Kontur der prominenten Silbe eingeschlossen wie bei Ratte:

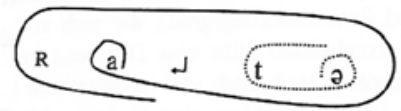

(Maas 1999, S. 198) 
Das Problem in dieser Darstellung besteht darin, dass bei festem Anschluss zwar eine „prominente Silbe“ - so der Maas'sche Ausdruck für die akzentuierte Silbe sowie eine „Folgesilbe [...] (die zwangsläufig eine Reduktionssilbe ist)“ angenommen wird, aber dennoch alles zusammen eine Silbe (s. Sievers Darstellung der Drucksilbe) ist - Maas spricht vom Einschluss „in die Kontur der prominenten Silbe“. Dabei bleibt unaufgeklärt, ob es sich hier um ein Phänomen allein von Akzentsilbe und Reduktionssilbe handelt, wie die Klammer bei Maas nahelegt, oder ob dies allgemein gilt, also auch beispielsweise bei Rettung, wo die zweite Silbe nicht als Reduktionssilbe interpretiert werden kann.

Was soll didaktisch gelten? Zuerst einmal muss man feststellen, dass beide Ansätze dieselben Phänomene erklären, also keine der beiden Theorien eine größere oder geringere Reichweite hat. Allerdings ist die Darstellung nach Wiese um einiges einfacher. Nicht nur werden nicht zwei verschiedene Silbenvorstellungen (Schallsilbe und Drucksilbe) ins Spiel gebracht, alle Phänomene können mit einem Modell erklärt werden, das, kurz gesagt, davon ausgeht, dass eine Akzentsilbe des Deutschen die Gestalt (K)KVK hat (wobei K nach V auch als Länge interpretiert werden kann).

Die Frage der Didaktik ist nicht, ob Drucksilben sinnvolle Annahmen sind, vielmehr, ob Drucksilben für das didaktische Geschäft eine sinnvolle Annahme sind, weil auf diese Weise Phänomene erklärt werden können, die didaktisch einschlägig sind.

Die Didaktik kann also feststellen, dass der sog. „silbenanalytische Ansatz“ mit Drucksilben einigen Aufwand bedeutet. Zwei verschiedene Silbenbegriffe denn der Ansatz braucht auch die Schallsilbe -, entsprechend ziemlich viele Häuschen- und Garagenmodelle. Dies sollte man nur dann übernehmen, wenn man mit dem alternativen Ansatz, der zumindest nach Occams Rasiermesser vorzuziehen ist, nicht weiterkommt.

Dies ist die Stunde der Lernenden und einer wirklich notwendigen Empirie. Wie gehen die Lernenden vor? Es gibt hierzu Untersuchungen (vgl. Berkemeier 2007). Auf zwei davon soll im Folgenden näher eingegangen werden.

Spiekermann (2002) untersucht den Einfluss des Dialekts auf die Schreibung im Schwarzwald (alemannisches Sprachgebiet), Hamburg (niederdeutscher Stadtdialekt) und Leipzig (sächsisches Dialektgebiet) bei ZweitklässlerInnen. Von der bisherigen Lerngeschichte erfährt man, dass die SchülerInnen mit einem Sprachbuch unterrichtet worden seien. Im Ergebnis stellt Spiekermann fest, dass, je nach Herkunft der SchülerInnen und den damit unterstellten dialektalen Sprechweisen, die SchülerInnen unterschiedlich „markierte und unmarkierte Schreibungen“ realisierten. Für diese Unterscheidung gilt die folgende Tabelle: 
Tab. 1: „Dehnungs- und Schärfungsschreibung - Modell“ (Spiekermann 2002, S. 198)

\begin{tabular}{|l|c|c|}
\hline & offene Silbe & geschlossene Silbe \\
\hline loser Anschluss & $\begin{array}{c}\text { unmarkiert } \\
<\text { Mole }>\end{array}$ & $\begin{array}{c}\text { markiert } \\
<\text { Huhn }>- \text {-Dehnung }\end{array}$ \\
\hline fester Anschluss & $\begin{array}{c}\text { markiert } \\
<\text { Sommer }>- \text { Schärfung }\end{array}$ & $\begin{array}{c}\text { unmarkiert } \\
<\text { Hemd }>\end{array}$ \\
\hline
\end{tabular}

Im Ergebnis zeigen sich erhebliche Unterschiede zwischen den drei untersuchten Standorten. Im Folgenden die Tabelle der markierten Schreibungen, wobei nun allerdings nicht mehr zwischen losen und festem Anschluss, sondern zwischen Kurzvokal (=fester Anschluss) und Langvokal (=loser Anschluss) unterschieden wird (vgl. hierzu Fußnote 1).

Tab. 2: Normgerechte Schreibung in \% bei sog. „markierten Fällen“ (Spiekermann 2002, S. 206)

\begin{tabular}{|c|c|c|c|c|c|c|c|c|}
\hline Ort & \multicolumn{2}{|c|}{ Kurzvokal } & \multicolumn{5}{c|}{ Langvokal } & Bemerkung \\
\hline- & offene S. & geschl. S. & \multicolumn{3}{|c|}{ offene S. } & \multicolumn{2}{c|}{ geschl. S. } & - \\
\hline- & - & - & +Son & -Son & $\#$ & +Son & -Son & - \\
\hline Schwarzwald & 7 & 33 & - & 0 & 0 & 0 & 20 & \\
\hline Hamburg & 47 & 36 & 0 & 0 & 67 & 0 & 29 & \\
\hline Leipzig & 70 & 78 & - & 0 & 100 & 75 & 22 & \\
\hline
\end{tabular}

Diese Unterschiede führt Spiekermann auf das dialektale phonologische System der Kinder zurück. Dies ist eine These, die allerdings weiter untersucht werden müsste. Besonders müsste dabei diskutiert werden, wie das stumme $<\mathrm{h}>$, das auf keine phonologische Eigenschaft rekurriert und zahlenmäßig bei etwa der Hälfte der einschlägigen Fälle überhaupt realisiert ist (weswegen es in einigen didaktischen Ansätzen als idiosynkratisch behandelt wird, weil es nicht vorhersagbar ist), von den Kindern so unterschiedlich behandelt wird. Wie auch immer man den Sachverhalt ansieht, das stumme $<\mathrm{h}>$ bzw. Dehnungs $-<\mathrm{h}>\mathrm{kann}$ nur per didaktischer Instruktion geschrieben werden. Das lässt darauf schließen, dass die $75 \%$ richtiger Schreibungen bei den Kindern aus Leipzig aufgrund des Unterrichts erreicht wurden und nicht aufgrund von interner phonologischer Regelanwendung. Hier ist sehr gut belegt, dass die Rechtschreibleistungen in den neuen Bundesländern höher waren als in den alten (vgl. Brügelmann 1994). 
Bei der sog. „Schärfungsschreibung“ müsste man ebenso den konkreten Unterricht kennen. Wurden die Kinder nach dem Modell von Maas, auf dem die Untersuchung von Spiekermann fußt, unterrichtet oder wurde ein Modell unterstellt, das ambisyllabische Konsonanten in der Aussprache realisiert oder wurde Rechtschreiben mehr oder weniger nach dem Reichen-Modell gelernt (was die Benutzung eines Sprachbuches nicht grundsätzlich ausschließen würde).

$\mathrm{Zu}$ der hier interessierenden Frage kann also die Untersuchung von Spiekermann (2002) wenig beitragen.

Anders die Ausführungen bei Huneke (2000), der sein Augenmerk auf Vorschulkinder lenkt. Seine Ausgangsfrage ist genau die hier besprochene:

Eine andere Position zur Didaktisierung der Schärfungsschreibung nehmen Maas (1990 u. 1992, S. 289) und Röber-Siekmeyer (1993, S. 80-83, S. 185-190 u. 1998) ein. Sie gehen ebenfalls von der Silbe aus und schlagen vor, das Phänomen abstrahierend bewusst zu machen und schon im frühen Rechtschreibunterricht explizit zu thematisieren; dabei setzen sie aber voraus, dass ein Bezug auf silbisches Sprechen didaktisch nicht sinnvoll ist, da der intervokale Konsonant in natürlicher gesprochener Sprache nicht als ambisyllabisch wahrnehmbar sei. Vielmehr würden Kinder spontan etwa Mu-tter, Pu-ppe oder Mu::-tter, Pu::-ppe syllabieren, zumindest im Falle der Okklusive, die nicht 'dehnbar' sind. Eine Silbifizierung wie Mut-ter und Pup-pe trete erst auf, wenn eine intensive Auseinandersetzung mit den entsprechenden geschriebenen Formen bereits stattgefunden habe; entsprechende sprachliche Produktionen oder Wahrnehmungen seien eine der typischen Übertragungen von Wissen über die Schreibung auf die gesprochene Sprache und ihre Wahrnehmung. Sie seien somit Ergebnis des Schreibenlernens und könnten deshalb nicht seine Grundlage sein. Eine zusätzliche Erschwerung stellten außerdem die dialektalen Verhältnisse im ehemals niederdeutschen Sprachgebiet dar. Um diesen beiden Argumenten gerecht zu werden, muss sich eine empirische Prüfung an Probanden wenden, die noch keine intensiveren Erfahrungen mit geschriebener Sprache gemacht haben, also an Vorschulkinder. Außerdem sollte sie nicht auf einen einzigen Regiolekt beschränkt bleiben.

Röber-Siekmeyer (1998) schließt eine didaktische Modellierung an, die auf eine unterrichtlich recht aufwendige Bewusstmachung des „Bremsens“ oder „Quetschens“ des betonten, ungespannten Kurzvokals abzielt.

Aus didaktischem Interesse ist es also interessant zu erfahren, ob sich Kinder mit einer ihnen gut zugänglichen sprachanalytischen Operation einen intuitiven Zugang zur Wahrnehmung ${ }^{2}$ des Silbengelenks im Deutschen verschaffen können, ohne vorgängig schon entsprechende Schreiberfahrungen gemacht zu haben. (Huneke 2002, S. 6)

2 Auf welche Sinne sich eine solche Wahrnehmung ggf. stützen kann, welche Form ihre Repräsentation hat und welcher Art resultierende sprachanalytische Wissensbestände und Kompetenzen sind, ist sehr schwer zu beurteilen. Aufgrund dieser Unsicherheit wird das Attribut intuitiv gewählt. [Fußnote bei Huneke]. 
Das Ergebnis:

Abb. 5: Vorschulkinder und Silbengelenk (Huneke 2002)

\begin{tabular}{|l|c|c|c|c|}
\hline \multirow{2}{*}{} & \multicolumn{2}{|c|}{ Nordbaden } & \multicolumn{2}{c|}{ Ostwestfalen } \\
\cline { 2 - 5 } & $\mathbf{N}=$ & $\%$ & $\mathbf{N}=$ & $\%$ \\
\hline Silbengelenk & 24 & $63 \%$ & 10 & $56 \%$ \\
\hline Silbengelenk oder keine Silbenzerlegung & 9 & $24 \%$ & 5 & $28 \%$ \\
\hline Kinder zerlegen mal nach Silben, mal anders & 5 & $13 \%$ & 3 & $16 \%$ \\
\hline Kinder zerlegen anders als nach Silben & 0 & $0 \%$ & 0 & $0 \%$ \\
\hline Summe & 38 & $100 \%$ & 18 & $100 \%$ \\
\hline
\end{tabular}

Als Ergebnis hält Huneke fest:

Grundschulkinder haben also einen intuitiven Zugang zum Silbengelenk. Sie können beim Syllabieren die Silbengelenke in zwei Komponenten aufspalten, und zwar unabhängig von einem Regiolekt und unabhängig davon, mit welchem Konsonanten ein Silbengelenk besetzt ist oder welcher Vokal vorausgeht. Diese Fähigkeit ist nicht erst ein Ergebnis der Begegnung mit Schrift, denn der weitaus überwiegende Teil von ihnen besitzt sie bereits vor Beginn des Schriftspracherwerbs; außerdem werden beim Sprechen auch die Silbengelenke aufgespalten, die in den Schreibungen gar nicht markiert sind. (Huneke 2000, S. 14)

Der Anteil derjenigen also, die ein Wort wie Hütte nicht in Silben zerlegen, ist ziemlich gering. Dies widerspricht der Fußnote 25 bei Maas, wo Maas zur Verteidigung des festen Anschlusses ausführt:

Das entspricht der gerade bei Grundschülern im Anfangsunterricht oft zu beobachtenden Unsicherheit, bei solchen Trochäen überhaupt zwei Silben wahrzunehmen, da die zweite Silbe (die fest angeschlossene Reduktionssilbe) ja eine Funktion in der prominenten Silbe erfüllt. Bei der beliebten didaktischen Methode des Silbenklatschens klatschen sie nur einmal [...]. (Maas 1999, S. 196)

Das Beispiel zeigt nicht nur, dass gelegentliche Beobachtungen, zumal wenn sie theoretisch präfiguriert sind, empirische Forschung in der Didaktik nicht ersetzen können. Für das Entscheidungsmodell, das oben für die Didaktik als leitend bezeichnet wurde, bedeutet das Ergebnis, dass die Wahrscheinlichkeit, dass die Kinder Hüt | te silbifizieren, ziemlich hoch ist. Der Schallsilbenansatz mit realisierten Silbengelenken setzt also am Können und Vermögen der Kinder an. Das Modell bietet daher ein sehr gutes Fundament für ein Weiterlernen.

Man hat also ein empirisches Ergebnis, das didaktisch plausibel interpretiert werden kann bzw. an das man didaktisch gut anknüpfen kann; zudem ist dieses 
Ergebnis auch unter Heranziehen des Occam'schen Rasiermessers begrüßenswert. Man braucht nur einen einzigen Silbenbegriff (Schallsilbe) und muss nicht offen oder implizit mit der Schall- und der Drucksilbe operieren.

Dies ist allerdings noch nicht alles, was hinsichtlich der Fassung fachlicher Inhalte unter einer didaktischen Perspektive zu bedenken ist. In Ossner (2015a, b) habe ich versucht, drei Stichwörter für die Beurteilung von didaktischen Handlungen in die Diskussion zu bringen: Erklärkraft, systematische Tiefe und Perspektivenwechsel.

- Erklärkraft: Die Gliederung in [hyt | tə] erklärt die Kürze des Tonvokals. Der Konsonant begrenzt den Vokal, der auf zeitliche Dauer angelegt ist. Daher werden die Vokale, wenn sie keinen Endrand haben oder isoliert gesprochen werden, grundsätzlich lang gesprochen.

- Systematische Tiefe: Beim Schallsilben-Modell haben (fast) alle Tonsilben die Form (K)KVK, wobei , $\mathrm{K}^{\mathrm{c}}$ hier auch als Morenposition gedeutet werden kann. Dadurch kann auch Länge als , $\mathrm{K}^{`}$ gezählt werden, was aus der Versmetrik hinlänglich bekannt ist. In diesem Sinne haben be: | tən und bet | tən nicht nur in der zweiten, sondern auch in der ersten Silbe dieselbe Strukturgestalt: KVK. Bis auf wenige Ausnahmen (Monde, Wüste, Erde, Pferde, Herde, Geste...) haben alle Tonsilben diese systematische Gestalt. Kinder können also auf dem einmal eingeschlagenen Weg selbsttätig weiterdenken, sie können relativ früh selbständig werden und brauchen nicht immer die führende Hand der Lehrkraft; dies scheint mir hinsichtlich des letzten Fundamentalziels von Unterricht, das meines Erachtens in der Emanzipation der zu Bildenden besteht, von einiger Bedeutung. Unter allen Theorieangeboten sollte m. E. didaktisch immer dasjenige siegen, bei dem der Lernende selbständig weiterdenken kann, weil er ein System an die Hand bekommen hat, das ihm dies ermöglicht. Die Kunst des Lehrens besteht dann darin, eine Schülerin/einen Schüler auf eine systematische Spur zu setzen, die ihm seinen eigenen Weg ermöglicht. Selbstverantwortete Eigenständigkeit ist der Ausdruck von Emanzipation.

- Kein unangekündigter Perspektivenwechsel: Zu den größten Problemen des Schriftspracherwerbs gehört ein ständiger Perspektivenwechsel. Mal wird die Angelegenheit von der Lautseite aus betrachtet, mal von der Schriftseite aus, ohne dass die Lernenden ausreichend darauf vorbereitet sind. An dem folgenden Zitat wird das Problem deutlich:

Schärfung (=Verdoppeln des Konsonanten, der zu Beginn der Silbe folgt) geschieht dann, wenn die betonte Silbe offen ist (=nicht mit einem Konsonanten endet) und einen kurzen Vokal hat. (Röber-Siekmeyer 1993, S. 89) 
In dieser Definition kommen ausschließlich phonologische Ausdrücke vor: Konsonant, (betonte) Silbe, Vokal. Also lautet die Anwendung dieser Regel auf ein Beispiel so: $[\mathrm{m} v \mid$ tər $] \rightarrow^{*}[\mathrm{~m} \mho \mid$ ttər$]$. Dies ist aber offensichtlich falsch. Gemeint ist allem Anschein nach etwas anderes: [motər $] \rightarrow[\mathrm{mo} \mid$ tər $] \rightarrow<$ Mu-tter $>$; verdoppelt wird bei dieser Sichtweise der Konsonantenbuchstabe, nicht der Konsonant. Diese Regel braucht im Übrigen den Zwischenschritt der Silbifizierung nicht, weswegen man den Ansatz in dieser Form auch nur sog. „silbenanalytischer Ansatz" nennen sollte. (Zu einer ausführlichen Darstellung des Ansatzes vgl. Spiekermann 2002.) In der vorgelegten Form unterscheidet sich das Ganze nur vom Aufwand her vom $₫ 2$ des Amtlichen Regelwerks:

Folgt im Wortstamm auf einen betonten kurzen Vokal nur ein einzelner Konsonant, so kennzeichnet man die Kürze des Vokals durch Verdoppelung des Konsonantenbuchstabens. $^{3}$

Während aber das amtliche Regelwerk klar zwischen Laut- und Schriftperspektive unterscheidet, geht dies bei Röber-Siekmeyer durcheinander. Hier wird stillschweigend von einer phonologischen Silbe zu einer graphischen übergewechselt, ohne diesen Schritt zu kennzeichnen. Dies macht die Angelegenheit für die Lernenden, die die Ebenenunterscheidung erst im Lernprozess nachzuvollziehen lernen, sehr schwierig.

Hinzu kommt, dass Schärfung phonologisch interpretiert werden kann: der Vokal bekommt eine scharfe Grenze; dagegen kann man aber nicht sagen, dass ein Vokal durch ein sog. „Dehnung-h“ lang würde. Vielmehr ist der Vokal schon lang. Die „Schärfungsregel“ kann aus der Perspektive des Schreibers formuliert werden, die „Dehnungsregel“ muss aber aus der Perspektive des Lesers oder der Leserin formuliert werden. Es wäre fatal, wenn jemand denken würde, dass Länge eines betonten Vokals, die er hört und spricht, in der Schrift durch $<\mathrm{h}>$ wiedergegeben würde.

Im Entscheidungsmodell heißt all dies, dass der Erwartungswert der Wünschbarkeit einer Handlung hoch anzusetzen ist, wenn Erklärkraft, systematische Tiefe und Perspektivität bedacht und eingehalten sind. Für diese Aussage gibt es einen

3 Beide Darstellungen haben ein besonderes Problem mit $\langle\mathrm{tz}\rangle$ und $\langle\mathrm{x}\rangle$; bei $\langle\mathrm{tz}\rangle$ wird argumentiert, dass dies eigentlich $<$ zz $>$ sei, so wie $<$ ck $>$ eigentlich $<$ kk $>$ sei. Für das Letztere hat man einen eindeutigen historischen Befund, denn die Schreibung $<\mathrm{ck}>$ geht auf Adelung (1788) zurück. Dagegen ist $<\mathrm{tz}>$ das Ergebnis einer Silbengelenkschreibung (vgl. Ossner 1996). Entsprechend der These müsste man auch annehmen, dass $<$ Hexe $>$ eigentlich ${ }^{*}<$ Hexxe $>$ sei, was dann irgendwie wieder im Gegensatz zu $<$ zZ $>$ vereinfacht worden sei. Hier sollte wiederum das Occam'sche Rasiermesser greifen. 
mehrfachen Grund. Lernen ist Selbsttätigkeit, die durch die drei Parameter gefördert wird und Selbsttätigkeit fördert Selbständigkeit, also die Emanzipation des Lernenden gegenüber dem/der Lehrenden. Damit bekommen Lehren und Lernen eine ethische Dimension.

Unterricht bewegt sich dabei zwischen Skylla und Charybdis. Skylla ist die falsche oder zu frühe Selbsttätigkeit. Dies ist beispielsweise bei dem von mir gewählten Gegenstandsbereich typisch für den Spracherfahrungsansatz, insbesondere die Reichen-Methode. Das Gefährliche an dieser Methode ist, dass sie offensichtlich die soziale Schere öffnet, weil diejenigen, die die helfende Hand der Lehrkraft bräuchten, diese nicht bekommen.

Charybdis ist die Behütung und Gängelung, die Selbsttätigkeit verhindert, typisch der schon lange beschriebene Fibeltrott.

Beide - Spracherfahrungsansatz und Fibeltrott - nehmen den Lehrkräften Entscheidungen an der falschen Stelle ab. Wünschenswert wäre Unterrichtsmaterial, das den Lehrkräften Entscheidungen vor Ort ermöglicht. Dies kann, wie oben schon angedeutet, nur dann der Fall sein, wenn die Lehrkräfte Entscheidungsparameter erkennen und das Material so durchsichtig ist, das es angepasst werden kann, sodass die Lehrkräfte vor Ort den Unterricht prozessieren können.

\section{Zusammenfassung}

Zusammenfassend kann man den Gedankengang hinsichtlich der Fassung eines fachlichen Inhalts (hier des Silbenkonzepts) unter einer didaktischen Perspektive also so darstellen:

Abb. 6: Die Aufgabe der Sprachdidaktik

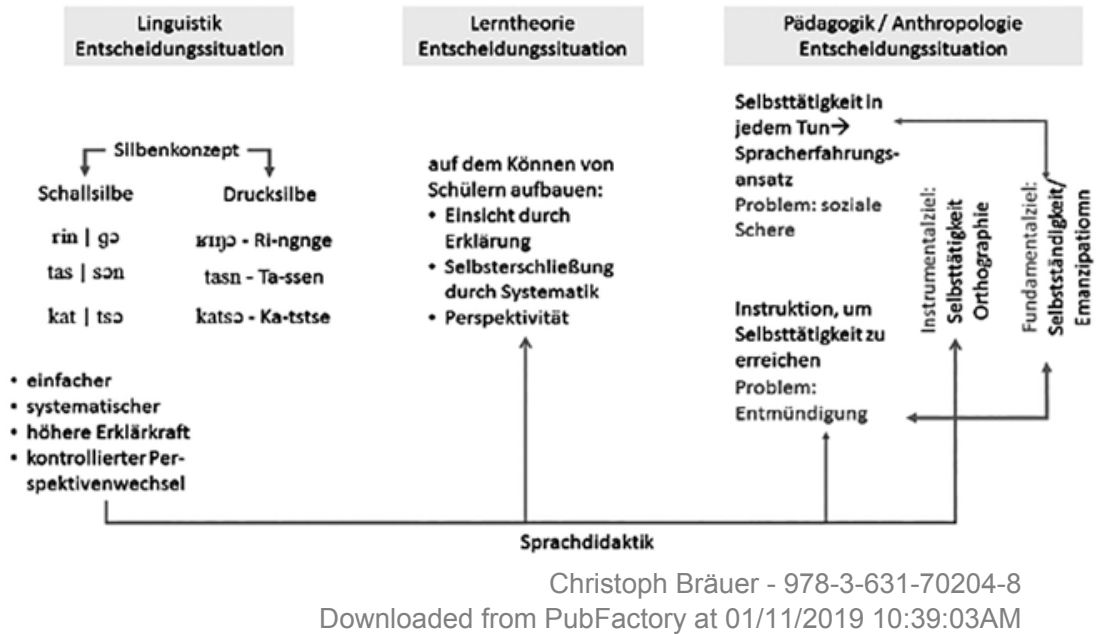


Damit sollte klar sein, was man sich unter einer didaktischen Konstruktvalidität vorstellen kann. (Was hier für ein Gegenstandsfeld gezeigt wurde, ist im Grundsatz nicht anders bei der Konstruktkonzeption einer empirischen Untersuchung.)

Es sollte eine Aufgabe der Deutschdidaktik sein, den Lehrkräften klare Konstrukte an die Hand zu geben, keinesfalls Modelle, die sie einfach übernehmen sollen. Auf diese Weise können transparente Materialien erstellt werden, die, je klarer und ausgewiesener die Konstrukte sind, desto besser vor Ort in der Schule angepasst werden können.

(Wozu die Deutschdidaktik vermutlich nicht besonders viel sagen kann, ist m. E., wie diese Konstrukte im Unterricht über die reine Sachlogik und Grundpfeiler des Lernens hinaus prozessiert werden sollen. Eine Wissenschaft ist kein guter Ort, an dem man lernen könnte, wie man mit konkreten Personen und einer Institution umgeht. Auch hier müssen dann Entscheidungen getroffen werden, die aber auf einer anderen Ebene liegen. Die Lehrkräfte hierin zu leiten, dazu ist die Deutschdidaktik als Wissenschaft überfordert, aber man kann Studierende und Lehrkräfte in diesem Bereich coachen.)

Für die gegenstands- und lerntheoretisch orientierten Entscheidungen kann die Fachdidaktik viel Unterstützung geben. Verlässlich und transparent ist Material, wenn die Gegenstandskonstruktion absolut durchsichtig ist, insbesondere der Auswahlprozess. Es wäre schön, wenn die DidaktikerInnen nicht auch Denkkollektive bilden würden, sondern Denkkollektive in der Wissenschaft kritisch beurteilen würden hinsichtlich ihrer Brauchbarkeit im didaktischen Prozess. Die Denkkollektive der Fachdidaktik sollten an einer anderen Stelle angesiedelt sein. (Die Rolle der Selbständigkeit im Lernprozess ist z. B. ein solcher Ort.)

In der Konstruktkonstruktion selbst braucht es eine starke Empirie, um das Können von SchülerInnen, auf denen die Konstrukte aufbauen, als Ausgangsbasis feststellen zu können. Ohne Empirie sind Wahrscheinlichkeiten für das Eintreffen Handlungsfolgen unter maßgeblichen Bedingungen nicht einschätzbar. Allerdings geht es jetzt nicht darum, ob etwas wirkt, sondern ob es wirken kann. Lehrkräfte brauchen nicht das Versprechen, dass sich der Erfolg einstellt, weil sie genau dieses oder jenes tun - sie wissen eh um die Wahrheit des lateinischen Sprichwortes si duo idem faciunt, non est idem - sondern Konstrukte, die sie selbst durchschauen können, damit sie vor Ort Wirkungen bei ihren SchülerInnen erzeugen können. 


\section{Literatur}

Abraham, Ulf/Bremerich-Vos, Albert/Frederking, Volker/Wieler, Petra (Hrsg.) (2003): Deutschunterricht und Deutschdidaktik nach PISA. Freiburg: Fillibach. Adelung, Johann Christoph (1788): Vollständige Anweisung zur deutschen Orthographie nebst einem kleinen Wörterbuch für die Aussprache, Orthographie, Biegung und Ableitung. Leipzig. (Reprint: Hildesheim: Olms 1978).

Bangel, Melanie/Müller, Astrid (2014):ZurEntwicklung morphologischer Bewusstheit und basaler Lesefähigkeiten durch die Arbeit an Wort-(bildungs)strukturen. Erste Ergebnisse einer Interventionsstudie. In: Didaktik Deutsch. 19. Jg., H. 36, S. 42-63.

Berkemeier, Anne (2007): Die Bedeutung der Silbe in der neueren rechtschreibdidaktischen Diskussion: Versuch einer Synopse. In: (Schrift-)Spracherwerb und Grammati(kali)sierung, OBST. Jg. 2007. H 73, S. 81-96.

Bredow, Rafaela/Hackenbroch, Veronika (2013): Die neue Schlechtschreibung. In: Der Spiegel, 25. http://www.spiegel.de/spiegel/print/d-98091072.html. Abgerufen am 1.9.2015.

Brügelmann, Hans (1994): Rechtschreibung in freien Texten. Zur Entwicklung schriftsprachlicher Teilleistungen von Klasse 1 bis 4. In: Brügelmann, Hans/ Richter, Sigrun (Hrsg.): Wie wir recht schreiben lernen. 10 Jahre Kinder auf dem Weg zur Schrift. Lengwil: Libelle, S. 149-168.

Fingerhut, Karlheinz (2002): Die Evaluation des Leseverständnisses durch die PISA-Studie und der Literaturunterricht in der Sekundarstufe I. In: Deutschunterricht. Jg. 2002. H. 3, S. 39-45.

Furhop, Nana (2006): Orthographie. Heidelberg: Universitätsverlag Winter.

Hattie, John (2013): Lernen sichtbar machen. Überarbeitete deutschsprachige Ausgabe von Visible Learning, bes. v. W. Bleyl und K. Zierer. Baltmannsweiler: Schneider.

Huneke, Hans Werner (2000): Intuitiver Zugang von Vorschulkindern zum Silbengelenk. In Didaktik Deutsch. 5. Jg., H. 8, S. 4-17.

Huneke, Werner (2002): Intuitiver Zugang von Vorschulkindern zum Silbengelenk - eine Grundlage für den Erwerb der Schärfungsschreibung? In: Tophinke Doris/Röber-Siekmeyer, Christa (Hrsg.): Schärfungsschreibung im Fokus: zur schriftlichen Repräsentation sprachlicher Strukturen im Spannungsfeld von Sprachwissenschaft und Didaktik. Baltmannweiler: Schneider, S. 85-105.

Hurrelmann, Bettina (2002): Leseleistung - Lesekompetenz. Folgerungen aus PISA mit einem Plädoyer für ein didaktisches Konzept des Lesens als kultureller Praxis. In: Praxis Deutsch. Jg. 2002. H. 176, S. 6-18. 
Klotz, Peter (1996): Grammatische Wege zur Textgestaltungskompetenz. Tübingen: Niemeyer.

Maas, Utz (1990): Die Rechtschreibung entdecken - am Beispiel von Dehnung und Schärfung. In: Praxis Deutsch. Jg. 1990. H. 101, S. 9-12.

Maas, Utz (1992): Grundzüge der deutschen Orthographie. Tübingen: Niemeyer.

Maas, Utz (1999): Phonologie. Einführung in die funktionale Phonetik des Deutschen, Opladen: Westdeutscher Verlag.

Melenk, Hartmut (1998): Aspekte der Kommasetzung in der 8. Klasse. Ergebnisse eines Forschungsprojektes. In. Didaktik Deutsch. Jg. 1998. H. 4, S. 43-61.

Neubauer, Skadi/Kirchner, Sabine (2013): Rechtschreibförderung von Grundschülern unter Berücksichtigung individueller Lernvoraussetzungen: Zwei Evaluationsstudien im Vergleich. In: Didaktik Deutsch. Jg. 2013. H. 35, S. 44-61.

Ossner, Jakob (1996): Silbifizierung und Orthographie des Deutschen. In: Linguistische Berichte. Jg. 1996. H. 165, S. 369-400.

Ossner, Jakob (2015a): Grammatik im Studium. In: Köpcke, Klaus-Michael et al. (Hrsg.): Deutsche Grammatik im Kontakt. Berlin: de Gruyter, S. 63-84.

Ossner, Jakob (2015b): Anforderungen an fachliches Wissen in der Deutschdidaktik. In: Anselm, Sabine/Janka, Markus (Hrsg.): Vernetzung statt Praxisschock. Göttingen: Edition Ruprecht, S. 100-112.

Reuter-Liehr, Carola (2006/2008): Lautgetreue Lese- und Rechtschreibförderung. Bd. 3, 4. Bochum: Winkler.

Röber-Siekmeyer, Christa (1993): Die Schriftsprache entdecken. Weinheim: Beltz.

Röber-Siekmeyer, Christa (1998): Mut zum Abstrahieren. Das Angebot von orthographischen Strukturierungen beim Lesen- und Rechtschreibenlernen in der Grundschule und seine Annahme durch Kinder. In: Oomen-Welke, Ingelore (Hrsg.): „....ich kann da nix!“. Mehr Zutrauen im Deutschunterricht. Freiburg. S. 137-159.

Schulte-Körne, Gerd \& Mathwig, Frank (2001/2004): Das Marburger RechtschreibTraining. Ein regelgeleitetes Rechtschreibprogramm für rechtschreibschwache Kinder. Bochum: Winkler.

Spiekermann, Helmut (2002): Der Einfluss des Dialekts auf die Schreibungen von Grundschulkindern. Überlegungen im Anschluss an eine empirische Untersuchung zum Vokalismus. In: Tophinke, Doris/Röber-Siekmeyer, Christa (Hrsg.): Schärfungsschreibung im Fokus: zur schriftlichen Repräsentation sprachlicher Strukturen im Spannungsfeld von Sprachwissenschaft und Didaktik. Baltmannweiler: Schneider, S. 186-221. 
van Dijk, Teun A./Kintsch, Walter (1983): Strategies of discourse comprehension. New York: Academic Press.

Vennemann, Theo (1986): Neuere Entwicklungen in der Phonologie. Berlin: Mouton de Gruyter.

Wiese, Richard (2000): The Phonology of German. Oxford: Oxford Univ. Press.

Wimmer, Heinz/Hummer, Peter (1990): How german-speaking first graders read and spell: Doubts on the importance of logigraphic stage. In: Applied Psycholinguistics. Jg. 1990. H. 11, S. 349-368.

Yu, Saek T. (1992): Unterspezifikation in der Phonologie des Deutschen. Tübingen: Niemeyer. 
Christoph Bräuer - 978-3-631-70204-8

Downloaded from PubFactory at 01/11/2019 10:39:03AM

via free access 


\title{
Iris Winkler \\ Deutschdidaktik - eine Anwendungswissenschaft? German didactics - an applied science?
}

\begin{abstract}
The present article discusses how to bridge the perceived gap between scientific knowledge of the German didactics ("Deutschdidaktik") research field and the everyday challenges of teaching German at school. As knowledge cannot simply be transferred, the development of bridging principles is a crucial issue of "Deutschdidaktik".
\end{abstract}

\section{Das ungeklärte Verhältnis von Deutschdidaktik und Deutschunterricht}

Studierende und Lehrpersonen erwarten nicht selten eine unmittelbare Anwendbarkeit von fachdidaktischem Wissen im Deutschunterricht - bzw. sehen sich in dieser Erwartung regelmäßig enttäuscht. Diese enttäuschten Erwartungen treten zu Tage, wenn etwa eine Studentin an ihrem fachdidaktischen Begleitseminar zum Praxissemester bemängelt, dass das „wieder nur sinnlose Theorie ist, die uns für die Schule sowieso wieder genau nichts bringt" (Holtz 2014, S. 107). Sie treten zu Tage, wenn Ausbildungsverantwortliche in der Schule den berüchtigten Rat erteilen: „Vergessen Sie, was Sie an der Uni gelernt haben“ (vgl. auch Zabka 2012). Aber auch wenn Stimmen aus der Deutschdidaktik beklagen, die Disziplin habe, seit sie dabei sei, „sich als forschende Wissenschaft neu zu erfinden“ (Abraham 2012, S. 63), ihre Kernaufgabe vernachlässigt, nämlich „die lehramtsbezogene Kompetenzvermittlung“ (ebd.), dann zeigt sich die Unzufriedenheit in Bezug auf die Anwendbarkeit fachdidaktischer Forschungsbefunde.

Mein Beitrag geht von der These aus, dass sich die wissenschaftlichen Erkenntnisse der Deutschdidaktik nicht ohne Weiteres auf das Berufsfeld Deutschunterricht übertragen lassen. Das heißt nicht, dass die Deutschdidaktik nichts hervorbrächte, was für den Deutschunterricht einen gewissen Nutzen hätte. Nur wie genau es um die Beziehung von Deutschdidaktik und Deutschunterricht bestellt ist, das halte ich für klärungsbedürftig (vgl. auch Winkler 2015). Eine solche Klärung ist durchaus mehrfach adressiert. Denn unser Profil ist nicht nur für uns selbst zuweilen unscharf. Studierende und Lehrpersonen müssen wissen, was sie von der Disziplin erwarten können, die ihr Handlungsfeld zum Forschungsgegenstand hat. Aber auch innerhalb der Universität herrschen etwa 
in den Fachwissenschaften z. T. recht diffuse Vorstellungen von Fachdidaktik und ihren Zuständigkeiten.

Um es vorweg zu schicken: Ich vertrete durchaus die Auffassung, dass das, was die Deutschdidaktik als Wissenschaft an Erkenntnissen hervorbringt, die Grundlage für Schlussfolgerungen sein muss, die im Berufsfeld produktiv sind. Aber meine Formulierung deutet schon an, dass die Anwendungsbeziehungen nicht unkompliziert sind.

Der folgende Beitrag gliedert sich vor dem Hintergrund dieser Vorüberlegungen in drei Teile: Im ersten Teil zeichne ich das Dilemma der Deutschdidaktik zwischen ,Theorie' und ,Praxis' nach, will dabei aber der Falle entgehen, eine deutschdidaktische Nabelschau zu betreiben. Deshalb beziehe ich Diskussionen ein, die andere Disziplinen führen, die als Anwendungswissenschaften betrachtet werden (können). Mit der Didaktischen Rekonstruktion stelle ich ein Modell zur Diskussion, das ich für geeignet halte, zwischen den verschiedenen Ansprüchen zu vermitteln, denen sich die Deutschdidaktik ausgesetzt sieht bzw. selbst aussetzt. Bereits hier zeigt sich, dass produktive Anwendungsbeziehungen zwischen Deutschdidaktik und Deutschunterricht nicht durch Operationen an der Oberfläche zu haben sind, sondern Elaborationen in der Tiefe erfordern. Dies verdeutlichen auch die Beispiele im dritten Teil, die ein Schlaglicht darauf werfen, wie Studierende in Anwendungssituationen mit fachdidaktischem Wissen umgehen.

\section{Deutschdidaktik im Spannungsfeld zwischen Wissenschaft und Anwendungsansprüchen}

Die Deutschdidaktik steht nicht allein im Spannungsverhältnis zwischen Wissenschaft und Anwendungsansprüchen. In einer ähnlichen Situation sind, wie Schriewer (2003) feststellt, alle

Fächer, die sich nicht lediglich oder nicht primär, [sic] über theoretische Probleme und die Generierung von Erklärungswissen definieren, sondern gleichermaßen über soziale Funktionen und das Angebot von Handlungs- und Orientierungswissen. Fächer mit anderen Worten, die sich auf die großen gesellschaftlichen Teilsysteme richten - Politik, Wirtschaft, Erziehung, Recht bzw. Religion -, die deren Entwicklung analysierend, deutend und beratend begleiten und den in solchen Systemen tätigen Professionen handlungsrelevantes Wissen zur Verfügung stellen (Schriewer 2003, S. 43).

Für seine Disziplin, die Vergleichende Erziehungswissenschaft, stellt Schriewer in historischer Perspektive eine „Dauerdiskrepanz" fest, die durch die gegenläufigen Ziele einer „Verwissenschaftlichung der Theorie [...] und einer gerade nicht methodisch übersetzten Bedienung reformpraktisch-bildungspolitischer 
Relevanzerwartungen“ (Schriewer 2003, S. 45) entsteht. ${ }^{1}$ Schriewer betont, dass die Aufhebung dieser Diskrepanz die „Überbrückung unterschiedlicher Rationalitätsformen“ voraussetze und diese wiederum „rechtfertigungsfähige Brückenprinzipien“ (Schriewer 2003, S. 45). Auf das Fehlen entsprechender Brückenprinzipien führt Schriewer beobachtbare Fehlentwicklungen zurück, wenn etwa im Kontext der PISA-Rezeption

das komplexe Gefüge von [...] Empirie, erziehungswissenschaftlicher Theorie und bildungspolitischer Handlungsrelevanz kurzgeschlossen [wird] zu einer unvermittelten Direktbeziehung zwischen Empirie und Praxisrelevanz (Schriewer 2003, S. 45).

Für die vergleichende Erziehungswissenschaft konstatiert Schriewer, dass die unterschiedlichen Ausrichtungen von Theorie generierender Wissenschaft und reformorientierter Politikberatung zur Aufspaltung der Disziplin geführt hätten in einen eher wissenschaftlich orientierten Zweig und einen eher anwendungsorientierten Zweig. Diese Aufspaltung sei kein pädagogiktypischer Sonderfall, sondern auch in den anderen eingangs genannten Fächern zu beobachten (Schriewer 2003, S. 46).

Ob auch in der Deutschdidaktik theoretisch und empirisch ausgerichtete Wissenschaft einerseits und anwendungsorientierte Entwicklungsforschung andererseits zunehmend auseinanderfallen werden, bleibt abzuwarten. Mir kommt es an dieser Stelle auf die Relevanz so genannter Brückenprinzipien an. Brückenprinzipien ermöglichen eine angemessene Verbindung der Rationalitätsformen von Wissenschaft und praktischer Handlungsrelevanz und helfen, Übervereinfachungen zu vermeiden.

Die Bezeichnung ,Anwendungswissenschaft' für Disziplinen, die wie die Deutschdidaktik um die Qualität der Bezüge zwischen Wissenschaftswissen und Anwendungssituationen ringen, ist in diesem Kontext eher missverständlich. Mit dem Begriff Anwendungswissenschaft ${ }^{2}$ wird nämlich oft kontrastierend operiert, wenn es um die generelle Frage der Wissenschaftlichkeit der eigenen Disziplin geht. Lässt sich das, was man betreibt, (lediglich) als technisch-konkrete Anwendungswissenschaft oder als abstrakt-theoretische (Grundlagen-)Wissenschaft

1 Die Deutschdidaktik kennt solche Diskrepanzerfahrungen beispielsweise aus der Mitwirkung an der Erarbeitung von Bildungsstandards und Vergleichsarbeiten. Für diese Mitwirkung gilt die Deutschdidaktik einerseits aus bildungspolitischer Sicht mit guten Gründen für zuständig; andererseits aber wurden hier der nötige theoretische Diskurs und die wissenschaftliche Fundierung von Modellen oft von der forcierten Umsetzung bildungspolitischer Steuerungsmaßnahmen gewissermaßen, rechts überholt'.

2 Ich danke Jette Eisoldt und Ulrike Vorwald für ihre Recherchen in diesem Kontext. 
definieren? In einigen Kontexten wird der Begriff zwar positiv gebraucht, v. a. dann, wenn man die Interdisziplinarität oder die Praxisnähe der Disziplin hervorheben möchte. Fachhochschulen, „Universities of Applied Sciences“, werben nicht zuletzt mit unmittelbar anwendbaren Studieninhalten moderner neuer Studiengänge. Ansonsten erscheinen Anwendungswissenschaften aber eher als defizitäre Wissenschaften, die nur durch die enge Verbindung zur ,Mutterwissenschaft' existieren können. Im Zuge der Profilierung als selbstständige wissenschaftliche Disziplin haben insbesondere die Medizin, die Praktische Theologie und die Pädagogik versucht, sich des Etiketts der Anwendungswissenschaft zu entledigen. Teilweise lassen sich diese Bemühungen bis heute beobachten. In der Medizin und Pädagogik wird stattdessen nicht selten die Bezeichnung als praktische Wissenschaft bevorzugt (vgl. z. B. Alisch 1995; Maio 2011; Ponnath 1995; Wieland 1986). Für die Deutschdidaktik hat bereits Ossner (1993) diese Bezeichnung vorgeschlagen und sich damit zugleich entschieden gegen „Fehler eines rezeptologischen Verständnisses von Didaktik“ (Ossner 1993, S. 193) gewandt.

Auf ein weiteres Missverständnis, das mit der Bezeichnung Anwendungswissenschaft verbunden sein kann, verweist die Debatte in der Religionspädagogik. Deren VertreterInnen lehnen den Status der Anwendungswissenschaft eindeutig ab, und zwar mit Blick auf ihre fachwissenschaftlichen Nachbardisziplinen:

Das Vor-Urteil, mit dem die Religionspädagogik innerhalb der Theologie chronisch zu kämpfen hat, lautet: Die Inhalte religiöser Erziehung lege die Theologie fest, der effektive Transport der von der Theologie als richtig und wichtig fixierten Inhalte sei dann die Aufgabe der Religionspädagogik. Religionspädagogik wird in einem solchen Verständnis fremddefiniert als Anwendungswissenschaft. Wie die Versandabteilung einer Firma habe sie dafür zu sorgen, dass die fertig produzierte Ware vollständig und unversehrt beim Kunden - den Schüler/innen - ankomme. Die Theologie verkünde, was gelehrt werden muss, der Religionspädagogik obliege die effektive Übermittlung. (Porzelt 2009, S. 135; vgl. auch Boschki 2007, S. 26 f.)

Ähnliche Vorstellungen über die Deutschdidaktik kann man in den Fachwissenschaften der Germanistik finden (und in den Fachwissenschaften generell über die Fachdidaktiken). Der Literaturwissenschaftler Albert Meier etwa vertritt die Auffassung, dass Literatur- und Sprachwissenschaft nicht für eine Lehrerbildung zuständig seien, „die mit Recht auf Anderes zielt als die genuine Literatur- bzw. Sprachwissenschaft". Literatur- und Sprachwissenschaft zielten auf Wissensermittlung. Für Fragen der Wissensvermittlung und Optimierung des Unterrichts seien allein die Pädagogik und Fachdidaktiken zuständig. Die Fachdidaktik habe sich aus Meiers Sicht in erster Linie „um praktische Handlungsanleitungen zur Optimierung des Unterrichts“ zu bemühen (Meier 2012, S. 157). Das hieße in der 
Konsequenz tatsächlich, dass die Deutschdidaktik die „Versandabteilung“ der Germanistik für die Schule wäre.

In der Fachdidaktik dürfte demgegenüber Konsens darüber herrschen, dass die Deutschdidaktik eigenes Wissenschaftswissen generiert, das sich von dem der fachwissenschaftlichen Bezugsdisziplinen unterscheidet. Der zweite Konsens dürfte darin bestehen, dass sich dieses Wissen ganz allgemein gesprochen auf Lehr- und Lernprozesse im Deutschunterricht bezieht und insofern im $\mathrm{Zu}$ sammenhang zum Handlungsfeld steht.

Eine Frage allerdings bleibt dann nach wie vor bestehen: Wie genau ist diese Beziehung zwischen Wissenschaftswissen und Handlungsfeld zu konkretisieren? Anders formuliert: Wie lassen sich wissenschaftliche Erkenntnisse auf die Erfordernisse des Deutschunterrichts so ,anwenden', dass dadurch weiterführende Einsichten für die Praxis möglich werden? Die Frage zielt auf eine Metaebene. Denn sie geht davon aus, dass die Implementierung von Forschungsergebnissen in die Praxis nicht als einfache Transmission, sondern gleichsam als Übersetzung („translating research into practice“; Woolf 2008, S. 211) zu denken ist. Deren Gelingensbedingungen wiederum sind gleichfalls als Forschungsgegenstand relevant (vgl. Woolf 2008).

\section{Didaktische Rekonstruktion als Brückenprinzip}

Wenn die Deutschdidaktik auf der einen Seite Wissenschaft sein und auf der anderen Seite etwas in Bezug auf den Deutschunterricht Relevantes zu sagen haben will, braucht sie also Brückenprinzipien, die gleichfalls wissenschaftlich zu reflektieren sind. Ein solches Brückenprinzip darf auf der einen Seite nicht den Eindruck der Rezeptologie (vgl. z. B. Ossner 1993) erwecken, als könnten und müssten Erkenntnisse der Fachdidaktik in mundgerechten Häppchen - also als unmittelbar umsetzbare Handlungsempfehlungen - dargeboten werden. Ein solches Brückenprinzip muss auf der anderen Seite vermeiden, dass die im Deutschunterricht gegebene Komplexität so reduziert wird, als könnte man ihr allein durch den Import wissenschaftlicher Befunde beikommen.

Ein Modell, das sich meines Erachtens als Brückenprinzip eignet, ist das der Didaktischen Rekonstruktion (Kattmann et al. 1997; Komorek et al. 2013). Es kommt wenig aufregend in Anlehnung an didaktische Dreiecke daher und stammt ursprünglich aus der Naturwissenschaftsdidaktik. Das Modell hat sich an der Universität Oldenburg als Denkrahmen fachdidaktischer Promotionsprogramme bewährt, ist also ein Modell, das didaktische Forschungsfragen systematisieren hilft. Es ist zugleich ein Modell, das explizit auf die Verbesserung von Unterrichtspraxis und Lehrerausbildung zielt. D. h. das Konzept der Didaktischen 
Rekonstruktion dient als harmonisierende Klammer zwischen den Ansprüchen der Fachdidaktik, einerseits Wissenschaft und andererseits Unterrichtsentwicklung zu betreiben. Es hat zudem einen entschieden fachlichen Kern.

Bei der Didaktischen Rekonstruktion eines Unterrichtsgegenstandes werden drei wechselwirkende Teile eng aufeinander bezogen: fachliche Klärung, Erfassung von Schülervorstellungen und didaktische Strukturierung. (Kattmann et al. 1997, S. 4)

Abb. 1: Modell der Didaktischen Rekonstruktion (Komorek et al. 2013, S. 43)

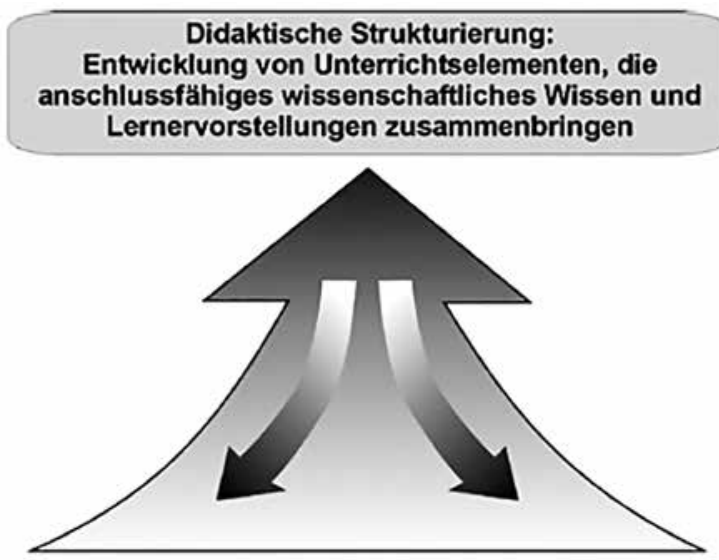

\section{Analyse und fachliche Klärung des domänen- spezifischen Wissens}

Empirische Untersuchung von Lernervorstellungen/ -vorwissen und von Lernprozessen

Dieses Aufeinander-Beziehen der „wechselwirkende[n]“ Aspekte macht tief gehende Informationsverarbeitung und damit anspruchsvolles didaktisches Denken erforderlich. Es widersetzt sich diametral der Erwartung, fachliches Wissen könne durch Anwendung methodischer Kniffe in Schülerköpfe implementiert werden.

Dass didaktische Argumentationen, die derart in der Tiefe ansetzen, keine eiligen Erwartungen von praktizierenden Lehrpersonen befriedigen, demonstriert

3 Ossner (1993) kommt zu einem sehr ähnlichen Schluss, wenn er - unter Einbeziehung der „Methode, wie die einzelnen Bausteine aufeinander zu beziehen sind“ (ebd., S. 197) - als fachdidaktische Wissensbereiche unterscheidet: Wissen über fachliche Gegenstände, Wissen über Lernende und Wissen über Handlungsoptionen. Vgl. auch das Modell fachdidaktischen Wissens von COACTIV (als Dreieck dargestellt bei Brunner et al. 2006, S. 60; vgl. auch Baumert/Kunter 2011a, S. 37 f.). 
sehr schön ein früher Aufsatz von Thomas Zabka. Zabka (1995) befasst sich mit der Systematisierung von Verfahren des handlungs- und produktionsorientierten Literaturunterrichts. Er verweist darauf, dass die Deutschdidaktik an der Oberfläche bleibt, wenn sie die Verfahren nach den geforderten äußeren Aktivitäten unterscheidet. Zabkas Leitfrage lautet stattdessen „Welche Arten des Verstehens ereignen sich bei welchen Formen produktiver Textbehandlung?" (Zabka 1995, S. 132).

Zabkas Darstellung der fraglichen Verfahren aus dieser Perspektive lässt zwar den Blick auf die Lernendenvorstellungen außen vor, der in der Didaktischen Rekonstruktion wesentlich ist. Seine Ausführungen sollen hier aber trotzdem als Beispiel für den Tiefgang didaktischer Rekonstruktionsprozesse angeführt werden; denn sie verdeutlichen zumindest, wie komplex allein das Wechselspiel zwischen fachlicher Klärung und didaktischer Strukturierung sein kann.

Beispielsweise lassen Lehrende den unterschlagenen Schluß einer Geschichte oft nur deshalb schreiben, weil die Erzählung auf unterschiedliche Weise enden könnte und Anlaß zu der Hoffnung besteht, die Lerngruppe werde die Handlung einigermaßen bereitwillig ausführen. Darüber hinaus wäre jedoch zu unterscheiden, ob beim Zuendeschreiben erstens die Sachgehalte eines Textes genau beachtet und verarbeitet werden sollen oder ob es zweitens vor allem auf den weitergeführten Stil des Textes ankommt; ob drittens primär deutlich werden soll, wie sich die Schreibenden einen Ort, eine Person, eine innere Sichtweise usw. vorstellen, oder ob durch das Ausdenken eines Schlusses die unterschiedlichen Auffassungen vom Sinn einer Geschichte manifest werden sollen. (Zabka 1995, S. 133)

Was hier verlangt wird, ist eine Basis soliden Wissens über unterschiedliche Gruppen von Textverstehensoperationen bzw. -zielen. Diese sind mit dem Wissen über fachliche Gegenstände, also literarische Texte, ins Spiel zu bringen und daraus - nicht zuletzt mit Blick auf die Lernenden, der im Beispiel fehlt Verstehensziele für den Literaturunterricht abzuleiten. Erst unter Einbeziehung dieser Überlegungen kann man sich für das eine oder andere handlungs- oder produktionsorientierte Verfahren und damit für die Strukturierung des Unterrichts entscheiden.

Das Modell der Didaktischen Rekonstruktion taugt als Brückenprinzip zur Vermittlung zwischen Wissenschaft und Deutschunterricht ebenso wie zur Vermittlung zwischen Forschungs- und Anwendungsorientierung der Deutschdidaktik. Im Oldenburger Promotionsprogramm ProfaS (Prozesse fachdidaktischer Strukturierung; vgl. Komorek et al. 2013) wurde das Modell auf die Strukturierung von Lerngelegenheiten für (angehende) Lehrpersonen bezogen. Dies unterstreicht, dass fachdidaktische Lehre ihrerseits die Vorstellungen und Wissensbestände ihrer Zielgruppe nicht vernachlässigen darf. Das Modell eignet sich also auch für die Einbeziehung der oben erwähnten zweiten Ebene von Anwendungsproblemen 
fachdidaktischen Wissens, nämlich die Diskussion der Frage, wie fachdidaktisches Wissen für Studierende und Lehrpersonen ,übersetzt' werden kann. Auf diese Problematik zielen die folgenden Beispiele, die fachdidaktische Lehre im Kern berühren.

\section{Anwendung fachdidaktischen Wissens im Studium}

In der Jenaer Deutschdidaktik bemühen wir uns, bei unseren Studierenden Offenheit und Neugier für Forschungsbefunde zu wecken. Wir möchten, dass die Studierenden lernen, einen Zusammenhang zwischen Wissenschaft - ,Theorie' und ,Praxis' zu sehen, ohne dass sie einfache Anwendungsbezüge herstellen. In den mündlichen Prüfungen, die wir regelmäßig abnehmen, antworten die Studierenden dann auch z. B. ganz wie erwünscht, sie würden ihre Kenntnisse von Lesekompetenzmodellen künftig für die Diagnose und Förderung von Lesekompetenz einsetzen. So weit, so gut - nur bleiben solche Pauschalantworten ganz an der Oberfläche. Ob komplexe Rekonstruktions- und Elaborationsprozesse gelingen, ist damit noch nicht gesagt. Ich möchte durchaus selbstkritisch zeigen, dass wir als Lehrende in der Fachdidaktik viel mehr darauf achten müssen, dass Argumentationen die Oberflächenebene verlassen und die Tiefenebene erreichen. Und wir müssen uns (wieder?) stärker bewusst machen, dass fachdidaktische Expertise nicht unabhängig von fachlicher Expertise sein kann (vgl. hierzu auch Baumert/Kunter 2011b, S. 185). ${ }^{4}$

Die folgenden Beispiele stammen aus einer Pilotstudie zu einer Untersuchung, in der es darum geht, fachdidaktische Kompetenzen von Studierenden zu fördern (Winkler et al. 2016). 28 Deutschstudierende in einem deutschdidaktischen Staatsexamensmodul, also gegen Ende ihres Studiums, wurden gebeten, mehrere mögliche Lösungen für eine Schüleraufgabe anzugeben und diese Schüleraufgabe anschließend hinsichtlich ihrer Schwierigkeit für Lernende im 7. Jahrgang einzuschätzen (Abbildung 2). Die Schüleraufgabe verlangt, zur Phädrus-Version der Fabel von Wolf und Lamm die Lehre zu ergänzen. Es handelt sich um die Fabel, in der der Wolf das Lamm beim Trinken am Bach mit unhaltbaren Vorwürfen überzieht und es frisst, bevor das Lamm auch den letzten Vorwurf entkräften kann.

4 Die Frage, wie viel Fachwissenschaft Lehramtsstudierende brauchen, ist bei der Konzeption von Studiengängen meiner Erfahrung nach nicht unumstritten. In Zeiten, in denen beispielsweise Ausbildungsinhalte zur Inklusion ins Lehramtscurriculum einbezogen werden müssen, ist immer auch zu diskutieren, auf Kosten welcher anderer Inhalte das gehen soll. Ein Beispiel, wie Fachwissenschaft lehramtsspezifisch ins Studium integriert werden kann, stellen Freudenberg et al. (2014) vor. 
Hierzu eine Lehre zu formulieren, ist eine schwierige Aufgabe für die genannte Altersgruppe - und nicht nur für diese. Die Schwierigkeit der Aufgabe liegt vor allem in einem unüblichen Moment des Textes: Weder siegt auf Handlungsebene die Tugend, d. h. das Lamm, über die Ungerechtigkeit noch wird das Handeln des Wolfes am Ende in irgendeiner Weise bestraft. Das Lamm ist also nur der moralische Sieger. Zu erkennen, dass diese Fabel also nicht (wie sonst oft) eine einfache Verhaltensregel zum besseren Miteinander transportiert, sondern ein (moralisch verwerfliches) komplexes gesellschaftliches Phänomen thematisiert, erfordert ein Maß an Abstraktionsvermögen, das eine 7. Klasse vor echte Herausforderungen stellt. Damit verstößt die Fabel auch gegen die Erwartungen, welche die Lernenden aus ihrer bisherigen Beschäftigung mit Fabeln gewonnen haben dürften.

\section{Abb. 2: Aufgabenstellung für Studierende}

Bei der folgenden Fabel fehlt die Lehre. Ergänze sie.

\section{Phädrus: Wolf und Lamm}

Wolf und Lamm waren an denselben Bach gekommen, um ihren Durst zu stillen. Stromaufwärts stand der Wolf und weit unterhalb das Lamm. Da brach der Räuber aus böser Fressgier einen Streit vom Zaun: ,Warum “, fragte er, „, hast du mir das Wasser aufgewühlt, das ich trinke? " Das Wolltier entgegnete bang: ,Wie, bitte schön, könnte ich fertigbringen, worüber Ihr Klage führt, Herr Wolf? Das Wasser, das ich trinke, kommt von Eurer Trinkstelle zu mir herabgeflossen. “

Nachdem er diesmal an der Kraft der Wahrheit gescheitert war, begann der Wolf von neuem: , Vor einem halben Jahr hast du schlecht von mir gesprochen. “ Lamm erwiderte: „Da war ich nicht einmal geboren. “,Dann“, sagte der Wolf, ,, hat eben dein Vater schlecht von mir gesprochen. "Und mit dieser Anschuldigung reißt er das Lamm, das gegen alles Recht sterben muss.

Diese Fabel zeigt...

Aufgabe an Studierende:

a) Geben Sie drei Lösungsmöglichkeiten an.

b) Analysieren Sie die Anforderungen der Aufgabe, wenn sie für Siebtklässler gestellt wird. 
Studierendenantwort 1: Begriffe ohne Begriff
a)
1) Macht und Willkür ergeben Unrecht.
2) Selbstverleumdung schützt in Zeiten der Gewalt.
3) Widerstand ist zwecklos.
b)

Das Aufgabenniveau ist für die 7. Klasse zu hoch. Ein Evozieren einer Lehre/Moral erfordert neben komplexen Fähigkeiten der Schüler auf Prozessebene hohe kognitive Anforderungen in der Herstellung von globaler Kohärenz und Superstrukturen (gerade diese!) in Verbindung mit ihrer Lebenswirklichkeit. Die Antworten der SuS zeigen, dass der Tenor dieser Fabel nicht erkannt wurde. Siebtklässler haben, denke ich, nicht das notwendige Hintergrundwissen (historisch), um speziell dieses Gleichnis in Verbindung mit Macht / Willkür / Selbstverleumdung / Widerstand (wenn ja: aktiv oder passiv) zu bringen.

Das erste Beispiel zeigt zunächst, dass der oder die Studierende selbst mit der Lösung der Schüleraufgabe nicht zurechtkommt. Der Text wurde anscheinend nicht verstanden. Die angegebenen Lösungsvarianten passen nicht zur Fabel. Lösungsvariante 1 ist ein Allgemeinplatz, den man immerhin in Verbindung zur Fabel bringen könnte. Variante 2 zeugt von Missverstehen; denn weder verleumdet sich das Lamm selbst, noch hätte ihm Selbstverleumdung helfen können. Variante 3 lässt fraglich erscheinen, ob verstanden wurde, wie Fabeln ,funktionieren' geschweige denn worum es in dieser Fabel geht.

Bei der Einschätzung der Aufgabenanforderungen fallen Begriffe, die in fachdidaktischen Lehrveranstaltungen in entsprechenden Zusammenhängen eine Rolle gespielt haben: Prozessebene, globale Kohärenz, Superstrukturen. Es wurden also immerhin Fachbegriffe behalten und im passenden Kontext abgerufen. Allerdings fehlt hier das Verständnis der Begriffe - sie werden unpassend und zusammenhangslos eingestreut, ohne dass dadurch etwas für die Einschätzung der Schwierigkeit dieser Schüleraufgabe gewonnen würde. ${ }^{5}$

Für die Fachdidaktik resultiert aus diesem Beispiel die Frage, inwiefern fachdidaktisches Wissen überhaupt eine Rolle spielen kann, wenn die fachlichen Gegenstände nicht durchdrungen werden. Was hätte es in diesem Fall genützt, wenn die aktivierten Begriffe richtig benutzt worden wären?

5 Ossner (1993, S. 194) spricht unter Rückgriff auf Wygotski von Pseudobegriff, um derartige Fälle zu bezeichnen, in denen „die begriffliche Terminologie zwar vorhanden ist, ohne daß der wissenschaftliche Begriff, also die souveräne Zugriffsweise, gegeben wäre“. 


\section{Studierendenantwort 2: Abruf und Anwendung von Wissen}

a)

- Streitsuchenden sollte man aus dem Weg gehen

- bei offensichtlichem Unrecht dennoch Recht geben, und die Beine in die Hand nehmen

- wenn der Wolf frisst, ist man besser weit weg

- wenn ein Grund gefunden werden muss, wird ein Grund gefunden

b)

- Text muss verstanden sein, um Aufgabe bearbeiten zu können

- sehr umfassende Aufgabe

- zuvor sollten andere Aufgaben mit geringerem Schwierigkeitsgrad gestellt werden

- Fabeln wurden im Jahrgang 5/6 behandelt $\rightarrow$ d. h. SuS sollten Gattungswissen haben $\rightarrow d$. h. sie müssten prinzipiell wissen, wie die Lehren in Fabeln formuliert sind $\rightarrow$ selbst $\mathrm{zu}$ formulieren dennoch schwer

- Integrationsgrad:

$\rightarrow$ es muss globale Kohärenz ausgebildet sein, um Aufgabe lösen zu können

$\rightarrow$ Aufgabe ist komplex, da alle Informationen nicht nur verknüpft, sondern auch bewertet/reflektiert werden müssen

- Entscheidungsspielraum:

$\rightarrow$ prinzipiell offen, da keine Wahlmöglichkeiten vorgegeben sind, aber dennoch nicht ganz so offen, da prinzipiell etwas ganz konkretes vom L erwartet wird

- Präzisionsgrad ebenfalls hoch

$\rightarrow$ Aufgabe ist sicherlich nicht für alle SuS zu leisten, gemeinsam in UG möglich

In diesem Beispiel gewinnt man zunächst einmal den Eindruck, dass die Fabel im Allgemeinen verstanden wurde. Die angegebenen Varianten für eine Lehre sind bis auf die letzte recht konkret und orientieren auf die Situation und Handlungsoptionen des Lamms. Dass hier der Mächtige versucht, Unrecht mit Recht zu verbrämen, wird nicht expliziert, klingt in der letzten Lösungsvariante aber immerhin an.

Sehr interessant im Kontext des vorliegenden Beitrags ist die Einschätzung der Aufgabenschwierigkeit in diesem Beispiel. Es wird nicht nur das Vorwissen der Lernenden in Bezug auf die Gattung Fabel bedacht. Es werden fast mustergültig Kriterien zur Einschätzung der Schwierigkeit von Textverstehensaufgaben genannt und erläutert. Auf diese Weise wird 'Stoff' aus den literaturdidaktischen Lehrveranstaltungen wiedergegeben. Anders als im ersten Beispiel sieht hier alles danach aus, als ob die Begriffe verstanden wurden. Es lässt sich also durchaus das Vorhandensein fachdidaktischen Wissens konstatieren. Diese/r Studierende hat gelernt, was gelernt werden sollte. Das Wissen wird auch korrekt , angewendet ${ }^{\mathrm{c}}-\mathrm{d}$. h. in der passenden Anforderungssituation 
abgerufen und Schritt für Schritt abgehakt. Es stimmt, dass in dieser Aufgabe Integrationsgrad und Präzisionsgrad hoch sind und der Entscheidungsspielraum auch, aber „nicht ganz so“.

Was ich zeigen möchte: Wir haben es hier mit einem Beispiel zu tun, in dem Wissensbestände oberflächlich entfaltet werden, ohne dass daraus in irgendeiner Weise Erkenntnis in Bezug auf das Aufgabenbeispiel erwachsen würde. Die Aufgabenlösung geht nicht über schematische Wissensbestände hinaus und benennt nicht genau, woraus in diesem Beispiel die Aufgabenschwierigkeit resultiert. Sie vernachlässigt komplett die Merkmale der vorliegenden Fabel. Was sie über die Schwierigkeit der Aufgabe feststellt, wäre in Bezug auf die Formulierung einer Lehre zu jeder beliebigen Fabel richtig, ja sogar in Bezug auf jede Aufgabe, einen Schluss zu einem literarischen Text zu verfassen. So allgemein, so wenig erhellend ist die Analyse. Denn sie ermöglicht es nicht, gezielt Schwierigkeiten der Lernenden $\mathrm{zu}$ antizipieren. In diesem Beispiel liegt Wissen vor und wird angewendet, aber es nützt nichts. Denn die listenartigen Applikation und Abarbeitung der erworbenen Wissensbestände bleibt an der Oberfläche, der tiefer gehende Blick für die Besonderheiten des Beispiels, die Auseinandersetzung mit dem fachlichen Gegenstand fehlt. Der Befund ist umso beunruhigender, als die in der Studierendenantwort dokumentierten Wissensbestände zu Prozessebenen des Textverstehens und schwierigkeitsbestimmenden Aufgabenmerkmalen aus fachdidaktischer Perspektive durchaus als hilfreich für Lehrpersonen erachtet werden (z. B. Maier et al. 2014; Rosebrock/Nix 2014; Winkler 2010).

Zwischen dem ersten und dem zweiten Beispiel besteht insofern eine Gemeinsamkeit, als die Studierenden sich mit der Demonstration erworbener Begriffe begnügen und weiterführendes Verstehen nicht anzustreben scheinen. Perkins (2008, S. 4; 6-8) spricht diesbezüglich von einer possessiven Haltung gegenüber Wissen. Zu hinterfragen ist, inwieweit die fachdidaktische Lehre möglicherweise dazu beiträgt, eine solche Haltung („spirit“, „attitude“; ebd., S. 8) eher zu festigen, statt zu überwinden.

Studierendenantwort 3: Rekonstruktion und Elaboration

a)

1. dass die Wahrheit (leider) nicht immer siegt

2. dass manchmal auch falsche, fadenscheinige Ausreden als Rechtfertigung für schlechte Handlungen dienen

3. dass das Recht des Stärkeren manchmal einfach durchgesetzt wird, ob mit oder ohne guten Grund

4. dass wo streitlustige Personen provozieren, jede Antwort die falsche ist, da sie den Streit nicht beilegen, sondern eskalieren lassen wollen 
b)

Die Anforderungen der Aufgabe sind für Siebtklässler zwar schon recht hoch, aber dennoch zu bewältigen. Die Aufgabe lehnt sich zwar eng an den Text an, lässt aber dennoch einen relativ großen Interpretationsspielraum. Dass es die Möglichkeit gibt, mehrere Lösungsmöglichkeiten zu finden, zeigt diesen Spielraum. Insofern besitzt die Aufgabe eine gewisse Offenheit. Von ihrer Komplexität ist sie aber durchaus zu bewältigen. Die Schüler müssen zwar eine vollständige Kohärenzetablierung leisten, um zu einer sinnvollen Lösung zu kommen (die Lehre stellt ja quasi die Interpretation dieser Fabel dar), aber der Text bietet sehr gut die Möglichkeit, sich an die individuellen Erfahrungen der Schüler zu richten. Zunächst ist die Konstellation Wolf-Lamm als ein klassisches, weithin bekanntes Jäger-Beute-Schema gut zu erkennen. Auch die Streitlust des Wolfes, dessen falsche und fadenscheinigen Gründe für den Angriff auf das (schwächere) Lamm könnten Schüler aus den eigenen Erfahrungen geläufig sein, z. B. Mobbing-Situationen oder auch das Streitgespräch mit Eltern oder älteren Geschwistern. Daher können die Schüler sich vermutlich relativ leicht vor allem in die Situation des Lammes hineinversetzen. Diesen Anknüpfungspunkt an konkrete, individuelle Erfahrungen der Schüler macht die Aufgabe durchaus lösbar für die Schüler, auch wenn sie ein intensives Nachdenken über den Text und vergleichbare Situationen ihrer Alltagswelt erfordern, in dem die metaphorische Kodifizierung von Wolf-Lamm als Täter-Opfer entschlüsselt werden kann.

Diese Antwort hat jemand verfasst, der den Text verstanden hat. Die angebotenen Lehren zielen auf den Kern des im Text dargestellten Problems ab. Auch die Analyse der Aufgabenschwierigkeit kann in diesem Beispiel überzeugen. Wieder fallen die nun schon bekannten Kriterien und Begriffe - hier: Offenheit, Komplexität, Kohärenzetablierung (schließlich ist die Antwort an die Fachdidaktikerin adressiert, von der anzunehmen ist, dass sie diese Begriffe hören will). Allerdings - und das ist der wesentliche Unterschied - verstellt die Kenntnis dieser Kriterien nicht den Blick auf die Besonderheiten des Textes und die zu erwartenden Vorwissensbestände der Lernenden. Diese/r Studierende hat verstanden, dass Aufgabenschwierigkeit nicht von der Textschwierigkeit und den Voraussetzungen der Lernenden zu lösen ist, und betreibt didaktische Rekonstruktion im besten Sinn: Die Konfliktsituation in der Fabel wird analysiert und auf die möglichen Erfahrungen der Lernenden bezogen. So wird die Grundlage für die didaktische Strukturierung der Arbeit mit dem Text im Unterricht geschaffen.

In unserer Stichprobe sind Lösungen dieser Qualität deutlich in der Minderzahl. Wir wissen letztlich nicht, warum sich die Studierendenantworten in ihrer Qualität so deutlich unterscheiden und warum die wenig befriedigenden Lösungen überwiegen. Für die fachdidaktische Lehre resultiert aus dem Befund auf jeden Fall die Notwendigkeit, (noch) stärker über geeignete Lernangebote 
nachzudenken, die das Potenzial haben, unterrichtsrelevante Erkenntnis dank fachdidaktischer Wissensbestände zu evozieren.

\section{Bilanz}

Fachdidaktisches Wissen ist noch nicht per se nützlich für unterrichtsnahe Anwendungssituationen. Nützlich wird es erst, wenn der Eigner dieses Wissens in der Lage ist, sich einen ,Begriff' davon zu machen und es dynamisch auf fachliche Gegenstände und SchülerInnenvorstellungen zu beziehen. Neben der Fähigkeit brauchen (angehende) Lehrpersonen die Bereitschaft („spirit“, „attitude“; vgl. Perkins 2008, S. 8), erworbenes Wissen selbstständig und kritisch zu ebenso konkreten wie produktiven Schlussfolgerungen zu nutzen. Diese anspruchsvollen Operationen müssen Lehrerpersonen selbst vornehmen - die Fachdidaktik kann und will sie nicht davon entlasten. Allerdings ist es Aufgabe der Fachdidaktik, Lernsituationen in Universität und Weiterbildung so zu gestalten, dass entsprechende Einsichten und eine fragend-reflektierende Grundhaltung angebahnt werden.

Der Anwendungsbegriff in Bezug auf die Fachdidaktik ist deshalb problematisch, weil er suggeriert, dass Wissensbestände - seien es fachwissenschaftliche oder fachdidaktische - 1:1 und unverändert ins Handlungsfeld transportiert bzw. darauf appliziert werden könnten. Zum Denkrahmen einer Fachdidaktik, die den Anspruch hat, als theoretische und empirische Wissenschaft etwas Relevantes in Bezug auf das Anwendungsfeld zu sagen, gehört es, Brückenprinzipien zu entwickeln; Brückenprinzipien, die es erlauben, verstehensfördernde Bezüge zwischen Wissenschaft und Praxis herzustellen. Ein solches Brückenprinzip kann das Modell der Didaktischen Rekonstruktion sein. Ob sich die Deutschdidaktik mit dem Etikett ,rekonstruktive' Wissenschaft gefällt, ist dann eine andere Debatte.

\section{Literatur}

Abraham, Ulf (2012): Literaturdidaktik und die Befähigung zur Teilhabe an der kulturellen Praxis. Literatur als Aufgabe der Lehrerinnen- und Lehrerbildung. In: Mitteilungen des Deutschen Germanistenverbandes. Jg. 59. H. 1, S. 59-72. Alisch, Lutz-Michael (1995): Grundlagenanalyse der Pädagogik als strenge praktische Wissenschaft. Berlin: Duncker \& Humblot.

Baumert, Jürgen/Kunter, Mareike (2011a): Das Kompetenzmodell von COACTIV. In: Kunter, Mareike/Baumert, Jürgen/Blum, Werner/Klusmann, Uta/Krauss, 
Stefan/Neubrand, Michael (Hrsg.): Professionelle Kompetenz von Lehrkräften. Ergebnisse des Forschungsprogramms COACTIV. Münster u. a.: Waxmann, S. 29-53.

Baumert, Jürgen/Kunter, Mareike (2011b): Das mathematikspezifische Wissen von Lehrkräften, kognitive Aktivierung im Unterricht und Lernfortschritte von Schülerinnen und Schülern. In: Kunter, Mareike/Baumert, Jürgen/Blum, Werner/Klusmann, Uta/Krauss, Stefan/Neubrand, Michael (Hrsg.): Professionelle Kompetenz von Lehrkräften. Ergebnisse des Forschungsprogramms COACTIV. Münster u. a.: Waxmann, S. 163-192.

Boschki, Reinhold (2007): Der phänomenologische Blick. „Vierschritt“ statt „Dreischritt“ in der Religionspädagogik. In: Boschki, Reinhold/Gronover, Matthias (Hrsg.): Junge Wissenschaftstheorie der Religionspädagogik. Berlin: LIT, S. 25-47.

Brunner, Martin/Kunter, Mareike/Krauss, Stefan/Klusmann, Uta/Baumert, Jürgen/Blum, Werner/Neubrand, Michael/Dubberke, Thamar/Jordan, Alexander/ Löwen, Katrin/Tsai, Yi-Miau (2006): Die professionelle Kompetenz von Mathematiklehrkräften: Konzeptualisierung, Erfassung und Bedeutung für den Unterricht. Eine Zwischenbilanz des COACTIV-Projekts. In: Prenzel, Manfred/ Allolio-Näcke, Lars (Hrsg.): Untersuchungen zur Bildungsqualität von Schule. Abschlussbericht des DFG-Schwerpunktprogramms. Münster u. a.: Waxmann, S. 54-82.

Freudenberg, Ricarda/Winkler, Iris/Gallmann, Peter/von Petersdorff, Dirk (2014): Von der Fachwissenschaft über die Fachdidaktik in den Schulunterricht und zurück - Ein Veranstaltungskonzept. In: Kleinespel, Karin (Hrsg.): Ein Praxissemester in der Lehrerbildung. Konzepte, Befunde und Entwicklungsperspektiven am Beispiel des Jenaer Modells der Lehrerbildung. Bad Heilbrunn: Klinkhardt, S. 162-176.

Holtz, Peter (2014): „Es heißt ja auch Praxissemester und nicht Theoriesemester“. Quantitative und qualitative Befunde. In: Kleinespel, Karin (Hrsg.): Ein Praxissemester in der Lehrerbildung. Konzepte, Befunde und Entwicklungsperspektiven am Beispiel des Jenaer Modells der Lehrerbildung. Bad Heilbrunn: Klinkhardt, S. 97-118.

Kattmann, Ulrich/Duit, Reinders/Gropengießer, Harald/Komorek, Michael (1997): Das Modell der Didaktischen Rekonstruktion - Ein Rahmen für naturwissenschaftsdidaktische Forschung und Entwicklung. In: Zeitschrift für Didaktik der Naturwissenschaft. Jg. 3. H. 3, S. 3-18.

Komorek, Michael/Fischer, Astrid/Moschner, Barbara (2013): Fachdidaktische Strukturierung als Grundlage für Unterrichtsdesigns. In: Komorek, Michael/ Prediger, Susanne (Hrsg.): Der lange Weg zum Unterrichtsdesign. Zur Be- 
gründung und Umsetzung fachdidaktischer Forschungs- und Entwicklungsprogramme. Münster u. a.: Waxmann, S. 39-58.

Maier, Uwe/Bohl, Thorsten/Drüke-Noe, Christina/Hoppe, Henriette/Kleinknecht, Marc/Metz, Kerstin (2014): Das kognitive Anforderungsniveau von Aufgaben analysieren und modifizieren können. Eine wichtige Fähigkeit von Lehrkräften bei der Planung eines kompetenzorientierten Unterrichts. In: Beiträge zur Lehrerinnen- und Lehrerbildung. Jg. 32. H. 3, S. 340-358.

Maio, Giovanni (2011): Mittelpunkt Mensch. Ethik in der Medizin. Ein Lehrbuch. Stuttgart: Schattauer.

Meier, Albert (2012): Zwei Eimer, also drei. In: Mitteilungen des Deutschen Germanistenverbandes. Jg. 59. H. 2, S. 156-158.

Ossner, Jakob (1993): Praktische Wissenschaft. In: Bremerich-Vos, Albert (Hrsg.): Handlungsfeld Deutschunterricht im Kontext. Frankfurt a. M.: Diesterweg, S. $186-199$.

Perkins, David (2008): Beyond Understanding. In: Land, Ray/Meyer Jan H.F./ Smith Jan (Eds.): Threshold Concepts within the Disciplines. Rotterdam/ Taipei: Sense Publishers, pp. 3-19.

Ponnath, Rainer (1995): Pädagogik als praktische Wissenschaft? Zur Möglichkeit wissenschaftlicher Orientierung pädagogischen Handelns im Anschluß an M. J. Langevelds Theorie der Erziehungssituation. Bad Heilbrunn: Klinkhardt.

Porzelt, Burkard (2009): Grundlegung religiöses Lernen. Eine problemorientierte Einführung in die Religionspädagogik. Bad Heilbrunn: Klinkhardt.

Rosebrock, Cornelia/Nix, Daniel (2014): Grundlagen der Lesedidaktik und der systematischen schulischen Leseförderung. [7., überarbeitete und erweiterte Auflage]. Baltmannsweiler: Schneider Hohengehren.

Schriewer, Jürgen (2003): Problemdimensionen sozialwissenschaftlicher Komparatistik. In: Kaelble, Hartmut/Schriewer, Jürgen (Hrsg.): Vergleich und Transfer. Komparatistik in den Sozial-, Geschichts- und Kulturwissenschaften. Frankfurt/New York: Campus, S. 9-52.

Wieland, Wolfgang (1986): Strukturwandel der Medizin und ärztliche Ethik. Philosophische Überlegungen zu Grundfragen einer praktischen Wissenschaft. Heidelberg: Winter.

Winkler, Iris (2010): Lernaufgaben im Literaturunterricht. In: Kiper, Hanna/Meints, Waltraud/Peters, Sebastian/Schlump, Stephanie/Schmit, Stefan (Hrsg.): Lernaufgaben und Lernmaterialien im kompetenzorientierten Unterricht. Stuttgart: Kohlhammer, S. 103-113.

Winkler, Iris (2015): Durch die Brille der anderen sehen. Professionsbezogene Überzeugungen im Lehramtsstudium Deutsch. In: Mitteilungen des Deutschen Germanistenverbandes. Jg. 62. H. 2, S. 192-208. 
Winkler, Iris/Heinrich, Matthias/Fischer, Astrid/ Krause, Ulrike-Marie (2016): Multiperspektivität in der Lehrerbildung. Fächerübergreifendes Lernen in der Mathematik- und Deutschdidaktik. In: Winkler, Iris/Schmidt, Frederike (Hrsg.): Interdisziplinäre Forschung in der Deutschdidaktik. „Fremde Schwestern" im Dialog. Frankfurt a. M.: Lang, S. 181-199.

Woolf, Steven H. (2008): The Meaning of Translational Research and Why It Matters. In: JAMA. Jg. 299. H. 2, pp. 211-213.

Zabka, Thomas (1995): Gestaltendes Verstehen. Zur Hermeneutik des gestaltenden Literaturunterrichts. In: Literatur in Wissenschaft und Unterricht. Jg. 28. H 2, S. 131-145.

Zabka, Thomas (2012): Der Trägheit des Lehrerwissens vorbeugen! In: Mitteilungen des Deutschen Germanistenverbandes. Jg. 59. H. 2, S. 179-181. 
Christoph Bräuer - 978-3-631-70204-8

Downloaded from PubFactory at 01/11/2019 10:39:03AM

via free access 


\title{
Deutschdidaktik - eine eingreifende Kultur- und kompetenzorientierte Vermittlungswissenschaft German didactics - a science of interfering culture and competence-oriented mediation
}

\begin{abstract}
The paper addresses the fields of action of German didactics spanning far-sighted extracurricular cultural sciences and a science of mediation that is embedded in a curricular context and researches in close proximity to real science. Building on that base, it outlines useful models and possible approaches to advancing the discipline in a contemporary manner.
\end{abstract}

\section{Denkrahmen, Denkstil und die Deutschdidaktik ${ }^{1}$}

Die Bestimmung des Denkrahmens der Deutschdidaktik und der Identität der Disziplin kommt einem allseits bemerkbaren Desiderat entgegen. Denn nach wie vor fällt der Mangel an einem konkreten, ausgeführten Denkrahmen und an Zielbestimmungen der Deutschdidaktik auf. So gibt es zwar zuletzt 2014 in „Didaktik Deutsch“ eine lebhafte Debatte zu den Bildungsstandards, aber ein programmatischer Beitrag wie Matthis Kepsers „Deutschdidaktik als eingreifende Kulturwissenschaft“ (2013) bleibt bis auf vereinzelte Reaktionen innerhalb der Deutschdidaktik fast völlig folgenlos und löst kaum eine produktive Debatte aus. ${ }^{2}$ Dabei lässt er sich für die Bestimmung eines deutschdidaktischen Denkrahmens nutzen.

Eine der ersten Fundstellen für den Begriff des Denkrahmens findet sich im Eröffnungsbeitrag für die Zeitschrift „Didaktik Deutsch“ aus der Feder von Hubert Ivo. Er bestimmt als

1 Für wertvolle Hinweise zu diesem Manuskript danke ich herzlich U. Abraham, A. Betz und N. Groeben.

2 Vgl. Müller-Michaels (2014), der an Fundierungen des kulturwissenschaftlichen Paradigmas der Deutschdidaktik erinnert, sowie Frederking (2014). 
Aufgabe einer allgemeinen Sprachdidaktik [...], einen Denkrahmen zu entwerfen, innerhalb dessen 1. die sprachdidaktischen Untersuchungsthemen modelliert und die Vorgehensweisen bestimmt, 2. die Rollen der Sprachdidaktik im Spannungsfeld von Wissenschaft und Schule reflektiert und 3. die für sie spezifischen, das Untersuchungsfeld erschließenden Zugänge gesucht werden können. (Ivo 1996, S. 8)

Nach Ossner (2001) steht der Denkrahmen in einem Wechselverhältnis mit dem Denkstil:

Ein Denkrahmen begründet die Außenlinien und damit das innere Feld, innerhalb dessen in einer Disziplin gedacht werden sollte. Ethische und anthropologische Überlegungen begründen einen Denkrahmen. Die rationale Entscheidungstheorie sowie ihre Schwester, die Spieltheorie, begründen einen Denkstil, indem es Wege und Abfolgen für Problemlösungen zeigt. (Ossner 2001, S. 29)

Denkstil in diesem Sinn impliziert die „klare Trennung zwischen den Zielen, der Erstellung von Zielsystemen und der Begründung von Handlungsalternativen zur Erreichung der Ziele“ (ebd., S. 29). Der Denkstil ermöglicht im Kontext eines Denkrahmens operative Verfahren der Zielbestimmung, der Entscheidungsfindung und des Abgleichs mit empirischen Evidenzen. Für Ossner ist "für das Funktionieren eines Denkstils das Vorhandensein von Zeitschriften, Lehr- und Handbüchern [sowie das Vorhandensein von] substantiellen Auseinandersetzungen [unabdingbar]" (ebd., S. 23). 2001 sollte diese Feststellung darauf hinweisen, dass Hand- und Lehrbücher in der Deutschdidaktik weitgehend fehlen und dass in deutschdidaktischen Zeitschriften keine substanziellen Auseinandersetzungen geführt werden. Beides ist heute, 15 Jahre später, nicht mehr der Fall, da an umfangreichen Hand- und Taschenbüchern kein Mangel herrscht, auch nicht an Lexika und Lehrbüchern sowie an zahlreichen, u. a. durch Jakob Ossner selbst angestoßenen substanziellen Auseinandersetzungen z. B. zur Kompetenzorientierung. ${ }^{3}$ Schließlich werden Denkrahmen und -stile typischerweise in Vorworten, Einleitungen und bei empirischen Studien in Vorgehensbegründungen umrissen. Und auch an Empirie, selbst an literaturdidaktischer Empirie, herrscht kein Mangel, obwohl die gerade hier nötige „Breite“ noch nicht erreicht ist. ${ }^{4}$

3 Zu einem deutschdidaktischen Lehrbuch vgl. Rupp 2014.

4 Noch immer hinken literaturdidaktische empirische Forschungen hinter der Lesedidaktik, der Sprach- und der Schreibdidaktik hinterher. 


\section{Zwei Konzeptionen der Deutschdidaktik: eingreifende Kultur- und kompetenzorientierte Vermittlungswissenschaft}

\subsection{Die Handlungsfelder der Deutschdidaktik}

Mein eigener Vorschlag eines Denkrahmens geht von Kepsers Konzeption einer eingreifenden Kulturwissenschaft aus. Kulturwissenschaftliche Bestimmungen der Deutschdidaktik haben eine lange Tradition, die Kepser z. T. wieder aufgreift. ${ }^{5}$ Deutschdidaktik teilt nach Kepser

insbesondere mit den Cultural Studies angelsächsischer Prägung [...] das Anliegen, es nicht lediglich bei der Erfassung, Beschreibung und Erklärung kultureller Phänomene belassen zu wollen, es geht auch um Veränderung (2013, S. 58).

Die Aufgaben der Deutschdidaktik bestehen demnach einmal in der Rekonstruktion des pädagogischen Wissens, Könnens und Handelns, aber zugleich in der kritischen Reflexion und Kommentierung von „Lehr-Lern-Prozessen als kulturellen Handlungen“ sowie in der Entwicklung von „Veränderungs- und Verbesserungsvorschlägen“" (ebd., S. 59).

Diese Aufgaben und ihre - erfolgreiche - Erledigung betreffen den Denkrahmen der Deutschdidaktik als Disziplin, und sie machen sozial-kommunikativ auch ihre Identität aus. Während der Denkrahmen den Theorie-Entwurf fokussiert, richtet sich die Identitätskonstruktion auf die Handlungsfelder der Deutschdidaktik, in denen sie sprachlich-literarische Lehr-Lern-Prozesse kommentiert und weiterentwickelt. Identität ist in der Sozialpsychologie ein Begriff, mit dem man ein dauerndes „inneres Sich-Selbst-Gleichsein“ (Erikson 1966, S. 124) des einzelnen Individuums im Kontext einer Gruppe bezeichnet hat. Sie besteht einerseits aus dem ,innere[n] Sich-Selbst-Gleichsein“ und andererseits aus dem "dauernde[n] Teilhaben an bestimmten gruppenspezifischen Charakterzügen" (ebd.). Diese gruppenspezifischen Charakterzüge bestehen bei der Deutschdidaktik durch die sozialen Handlungsfelder der Deutschdidaktik bestimmt, in denen sie agiert. Diese Handlungsfelder hängen zwar miteinander zusammen, lassen sich aber sachlogisch voneinander unterscheiden und unterliegen unterschiedlichen Zwängen und Bedingungen. Im Einzelnen sind dies:

5 Vgl. Baum/Bönninghausen 2013; Ingendahl 1991; Kepser 2013, S. 59; Müller-Michaels 2014, S. 120. 
Tab. 1: Übersicht über einige exemplarische Handlungsfelder der Deutschdidaktik und des Deutschunterrichts

\begin{tabular}{|l|l|l|l|}
\hline \multicolumn{1}{|c|}{ Universität } & \multicolumn{1}{|c|}{$\begin{array}{c}\text { (experimentelle) } \\
\text { Forschung }\end{array}$} & \multicolumn{1}{|c|}{$\begin{array}{c}\text { Schule: einzelne } \\
\text { Lerngruppen }\end{array}$} & $\begin{array}{c}\text { Schule als Institution } \\
\text { bzw. System }\end{array}$ \\
\hline \multicolumn{2}{|c|}{ Theorie } & \multicolumn{2}{|c|}{ Praxis } \\
\hline $\begin{array}{l}\text { Theoriearbeit, } \\
\text { Modellierung, } \\
\text { Lehrerbildung } \\
\text { Auswahl von } \\
\text { Gegenständen }\end{array}$ & $\begin{array}{l}\text { Operationalisierung, } \\
\text { Testung, Bewährung } \\
\text { in der Praxis }\end{array}$ & $\begin{array}{l}\text { Förderung und } \\
\text { Erforschung von } \\
\text { Unterricht und } \\
\text { Lehr-Lern-Prozessen }\end{array}$ & $\begin{array}{l}\text { Implementierung } \\
\text { Performanz } \\
\text { Entwicklung } \\
\text { Fortbildung/ } \\
\text { Ausbildung } \\
\text { (Lehrerseite)? }\end{array}$ \\
\hline
\end{tabular}

Deutschdidaktik beschäftigt sich zunächst in qualitativ-empirischer Hinsicht mit Theoriearbeit und mit Modellierungen, aus denen sie Maßstäbe für die Rekonstruktion (Beobachtung) und die Konstruktion von Unterricht gewinnt. Schon die Operationalisierung theoretischer Konstrukte ist zum Teil Praxis, weil die Konstrukte an die Zwänge empirischer Datenerhebung und-auswertung angepasst werden müssen (Rechenbarkeit, Stichprobengröße, Itemkonstruktion etc.) und die quantitativ-empirische Perspektive hinzutritt. Vollends praktisch und in gewisser Weise kontingent ist dann die Arbeit mit einzelnen Treatment- und Kontrollgruppen bei der Implementierung von Förderkonzepten, deren Erfolg von zahlreichen, nicht immer zu kontrollierenden weiteren Faktoren abhängt. Das Gleiche gilt in verstärktem Maße für die Veränderung der Schule als Institution, wobei die Zwänge jeder gesellschaftlichen Institution zum Tragen kommen. Als fünftes und letztes Feld wäre die Gesellschaft zu nennen, die letztlich Adressat deutschdidaktischer Forschung ist.

Gemäß der Identitätsbestimmung als „wechselseitiger Beziehung“ (Erikson 1966, S. 124) legt die Übersicht über die Felder der Deutschdidaktik einerseits ein Plädoyer für eine weitgehend autonome, unbeirrte Theorie-Arbeit aufgrund der bereichsspezifischen Trennung der Theorie-Arbeit von allen praktischen Handlungsfeldern nahe. Andererseits sollen sich die theoretischen Konstrukte der Praxiserprobung stellen, ohne dass sie in den Maßstäben der Praxis aufgehen oder gar von dieser fremdbestimmt werden. Deswegen sind weite DidaktikKonzeptionen sinnvoll, die eine distanzierte Position der Deutschdidaktik gegenüber Praxiszwängen zulassen. Andererseits muss der Rückbezug zur Praxis immer mitgedacht werden. 


\subsection{Deutschdidaktik als eingreifende Kulturwissenschaft}

Der Vorschlag Kepsers „Deutschdidaktik als eingreifende Kulturwissenschaft“ zu begreifen, lässt eine entsprechende Identitätskonstruktion der Deutschdidaktik durch das Aufspannen eines weit reichenden Denkrahmens zu. Er schließt Rekonstruktion, Konstruktion und Kritik pädagogischer Praxis mit ein und bezieht sich auf Schule und Unterricht als kulturelle Institutionen. Dabei ist der Fokus der Deutschdidaktik nicht nur auf die Verbesserung des Unterrichts ausgerichtet, sondern auch auf die qualitative Analyse der kulturellen Praktiken, die im Unterricht vollzogen werden, aber ihren eigentlichen Ort auch im Alltag und in der Gesellschaft haben. In besonderer Weise erforscht die Deutschdidaktik den Erwerb solcher kulturellen Praktiken, um den Deutschunterricht optimal auf solche vor- und außerschulischen Prozesse anzupassen. An Beispielen hierfür nennt Kepser u. a. die Ritualforschung, durch die untersucht wird, wie ganz allgemein Individuen ihre Identitäten konstruieren, aber auch fixieren. In diesem Lichte sind bestimmte Fragemuster, Aufgabenstellungen und auch Interpretamente z. B. im Literaturunterricht Rituale, durch die ästhetische Erfahrung evoziert, aber auch wieder reduziert wird.

Das Beispiel der Ritualforschung im Literaturunterricht zeigt die weite Untersuchungsrichtung, die für den kulturwissenschaftlichen Ansatz als einer Suchbewegung kennzeichnend ist und aus der (noch) keine unmittelbaren Schlussfolgerungen gezogen werden brauchen. Trotzdem sind dies wichtige deutschdidaktische Forschungsfelder. Dies öffnet den Blick dafür, dass die Deutschdidaktik als Expertin nicht nur für schulische, sondern auch für zahlreiche außerschulische Handlungsorte und Akteure zuständig ist, wo Auswahlprozesse stattfinden und wo Sprache und Kultur, Literatur und mediale Produkte dargeboten werden: also in den öffentlichen und privaten Print- und AV- sowie digitalen Medien, in Institutionen der Fort- und der Weiterbildung, in den Theatern und in den Museen sowie im Outdoor-Bereich. In letzerem können z. B. Wanderpfade begangen, mit Ausstellungsstücken ausgestattet und so als Orte der kulturellen Erinnerung erfahren und reflektiert werden. ${ }^{6}$

6 Vgl. Betz et al. 2013; Rupp 2008. 
Abb. 1: Außerschulische Orte der Vermittlung

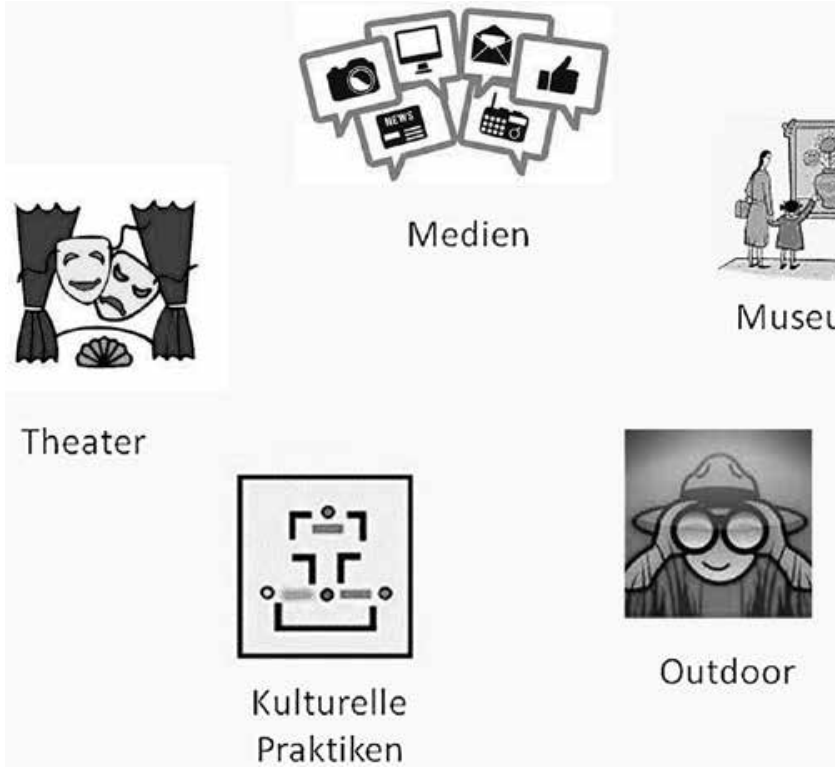

\subsection{Deutschdidaktik als kompetenzorientierte Vermittlungswissenschaft}

Der weiten kulturwissenschaftlichen Untersuchungsrichtung als anderer Pol gegenübergestellt ist der unmittelbare Bezug der Deutschdidaktik auf die Empirie-Orientierung und ihre Ausprägung als kompetenzorientierte Vermittlungswissenschaft. Hier bezieht sie sich auf das Lehren und Lernen in schulischen Kontexten. Deutschdidaktik erweist sich hier als angewandte Handlungswissenschaft, wie sie auch für Ingenieurwissenschaften und Sozialwissenschaften typisch ist. D. h.: Deutschdidaktik betreibt die (normengeleitete) Beobachtung, und Analyse von (schulischen) Lehr-Lern-Prozessen mit dem Ziel, aufdiese in konstruktiver Weise einzuwirken.

Die Beobachtung und Analyse von Lehr-Lern-Prozessen sind wissenschaftliche Tätigkeiten, die aufgrund der gegenwärtig gegebenen Empirie-Orientierung auf die Ermittlung derjenigen Faktoren ausgerichtet sind, die zu einer guten sprachlichen, literarischen und medialen Bildung aller SchülerInnen beitragen. Im Ensemble des didaktischen Dreiecks (übrigens eines alten didaktischen Denkrahmens) von LehrerIn, SchülerIn und Gegenstand sind dies Personen-, Prozess- und Gegenstandsvariablen. Bei den Personenvariablen handelt es sich Eigenschaften 
und Handlungsvollzüge von LehrerInnen und SchülerInnen, bei den Prozess- und Gegenstandsvariablen um die unterschiedlichen Schwierigkeitsgrade der jeweils gestellten Lernaufgabe und des jeweils ausgewählten Lerngegenstands. Wegen der Relevanz des fachlichen Lerngegenstands, aus dem die Deutschdidaktik ihre fachliche Identität herleitet, kann man auch von der fachorientierten Vermittlungswissenschaft sprechen.

Zum besseren Verständnis des Gesamtzusammenhangs und auch des konkreten Ortes, den die Kompetenzbestimmungen im Kontext der Deutschdidaktik einnehmen, dient die Übersicht über die Strukturelemente der Deutschdidaktik.

\section{Abb. 2: Strukturelemente der Deutschdidaktik ${ }^{7}$}
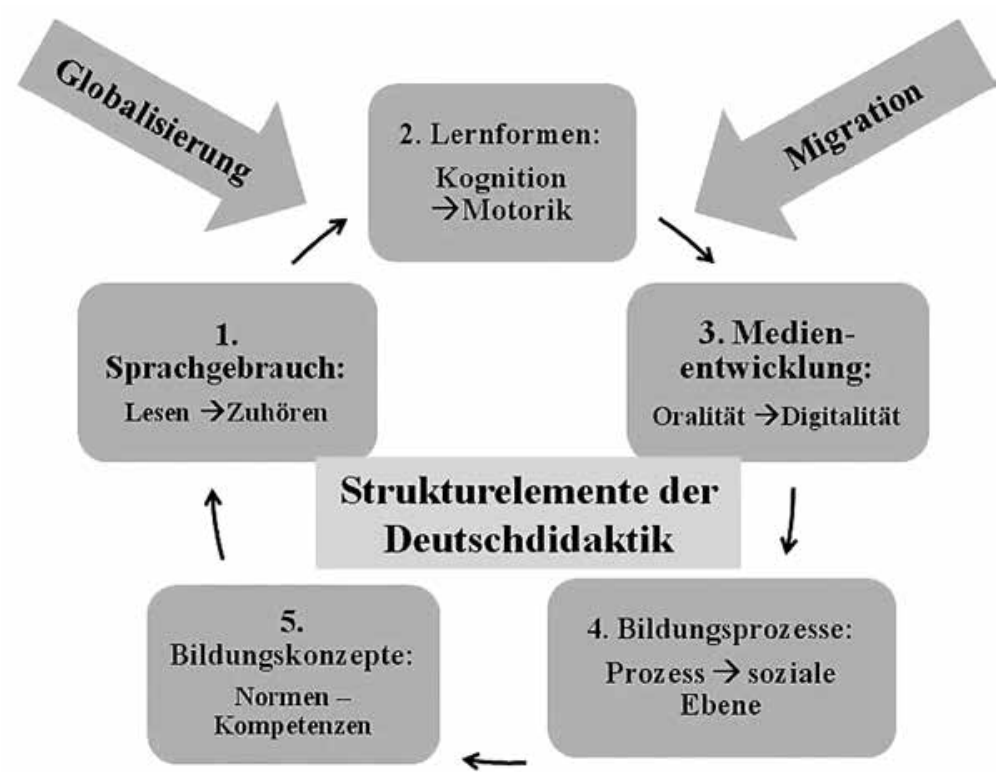

Deutschdidaktik bezieht sich auf die folgenden fünf Strukturelemente:

1. auf die Formen des Sprachgebrauchs vom Lesen bis zum Zuhören,

2. auf die unterschiedlichen Lern- und Verarbeitungsmodi,

3. auf die (medialen) Träger der Unterrichtsgegenstände,

4. auf die sozial unterschiedlichen Bildungsprozesse und

5. auf die wissenschaftlich unterschiedlich fundierten Bildungskonzepte. 
All diese Elemente werden durch Migration und Globalisierung moderiert. Die Abfolge spiegelt ihre zunehmende Komplexität und Relevanz. Die Elemente haben jeweils eine unterschiedliche Struktur: Dies sind die Formen des Sprachgebrauchs, die Lernziel-Taxonomie, der Zeitstrahl der Medienentwicklung, das Mehrebenenmodell von Bildungsprozessen von der Mikro- bis zur Makrobene und die polare Spannung bzw. wechselseitige Ergänzung zwischen Bildungsnormen und Kompetenzen. In dieser Vielfalt erweisen sich diese Strukturelemente, die in ihrer Summe einen Denkrahmen bezeichnen, als einzelne Formen von Denkstilen auf ganz unterschiedlichen Ebenen. Die Formen des Sprachgebrauchs erlauben die Zuordnung zu Lernbereichen, die Lernformen die Zuordnung zu einem bestimmten Verarbeitungsbereich, die medialen Träger die Zuordnung des Unterrichtsgegenstands zu Mündlichkeit bzw. Schriftlichkeit oder zur Medialität. Weiterhin können Bildungsprozesse und -diskurse auf der individuellen, institutionellen oder öffentlichen Ebene verlaufen und durch Normen und/oder Kompetenzen gesteuert werden.

Diese Strukturelemente bestimmen in ihrer Summe den Denkrahmen Deutschdidaktik, aber auch einzelne Unterrichtsvorhaben und -projekte. Dabei dient die Prüfung des eigenen Vorhabens der näheren Klärung der eigenen Position und der eigenen Zielstellungen.

Auch in diesem Modell sind Bestimmungen der Deutschdidaktik sowohl auf Kompetenzen wie auf Bildungsnormen ausgerichtet. Dabei gilt die Aufgabe, Bildungsvorstellungen wie z. B. die literarische Bildung weitestgehend zu operationalisieren. Andererseits sollten Kompetenzbestimmungen durch weitgefasste Bildungsvorstellungen wie Mündigkeit, Humanität und Selbstbestimmung inhaltlich präzisiert werden.

Um nun im Einzelnen bestimmte kompetenzorientierte Vermittlungsprojekte zu fundieren, betreibt die Deutschdidaktik eine ,realwissenschaftliche Forschung; bei der es um den bestmöglichen Sprach-, Literatur-, Kultur- und Medienerwerb geht. Wenn man die Deutschdidaktik wissenschaftssystematisch einordnen will, handelt es sich um eine Realwissenschaft im Gegensatz zu abstrakten Formalwissenschaften wie Philosophie, Logik oder Mathematik. Sie ist eine (sozialwissenschaftliche) „angewandte“ Handlungswissenschaft, bei der die

Analyse menschlicher Handlungsalternativen zwecks Gestaltung sozialer und technischer Systeme im Vordergrund [steht. Realwissenschaften] versuchen deshalb, Entscheidungsmodelle bzw. Entscheidungsprozesse zu entwickeln (Ulrich/Hill 1977, S. 305).

Die Entscheidungen beziehen sich auf die Auswahl der empirisch ermittelten optimalen Lehr- und Lernprozesse sowie der Lerngegenstände. Die Entschei- 
dungsprozesse werden in Prozessmodellen operationalisisert. Das Prozessmodell deutschdidaktischer Forschung umfasst sechs Teilphasen, in denen normativpräskriptive und analytisch deskriptive Anteile aufeinander bezogen sind. Den Ausgangspunkt bildet unter 1. eine normative Zielbestimmung, die z. B. in einer Kompetenzerwartung bzw. in bestimmten Hypothesen realisiert wird, und zugleich die Beschreibung der tatsächlichen Performanz, die deskriptiv beobachtet wird. Das Eingreifmodell unter 2. setzt bereits Schlussfolgerungen um, die aus dem Vergleich zwischen erwarteter und beobachteter Norm gezogen worden sind und die in eine neue u. U. kontrastive Versuchsanordnung eingehen. In der dritten Phase werden wie bei 1. die Lernergebnisse analysiert und auf die Normen bzw. die Hypothesen bezogen. In der 4. Phase wiederholt sich die Modellaufstellung bzw. -anpassung, die in der 5. und 6. Phase erprobt und verändert werden - worauf sich der Kreis schließt.

Abb. 3: Ein Prozessmodell realwissenschaftlicher Forschung

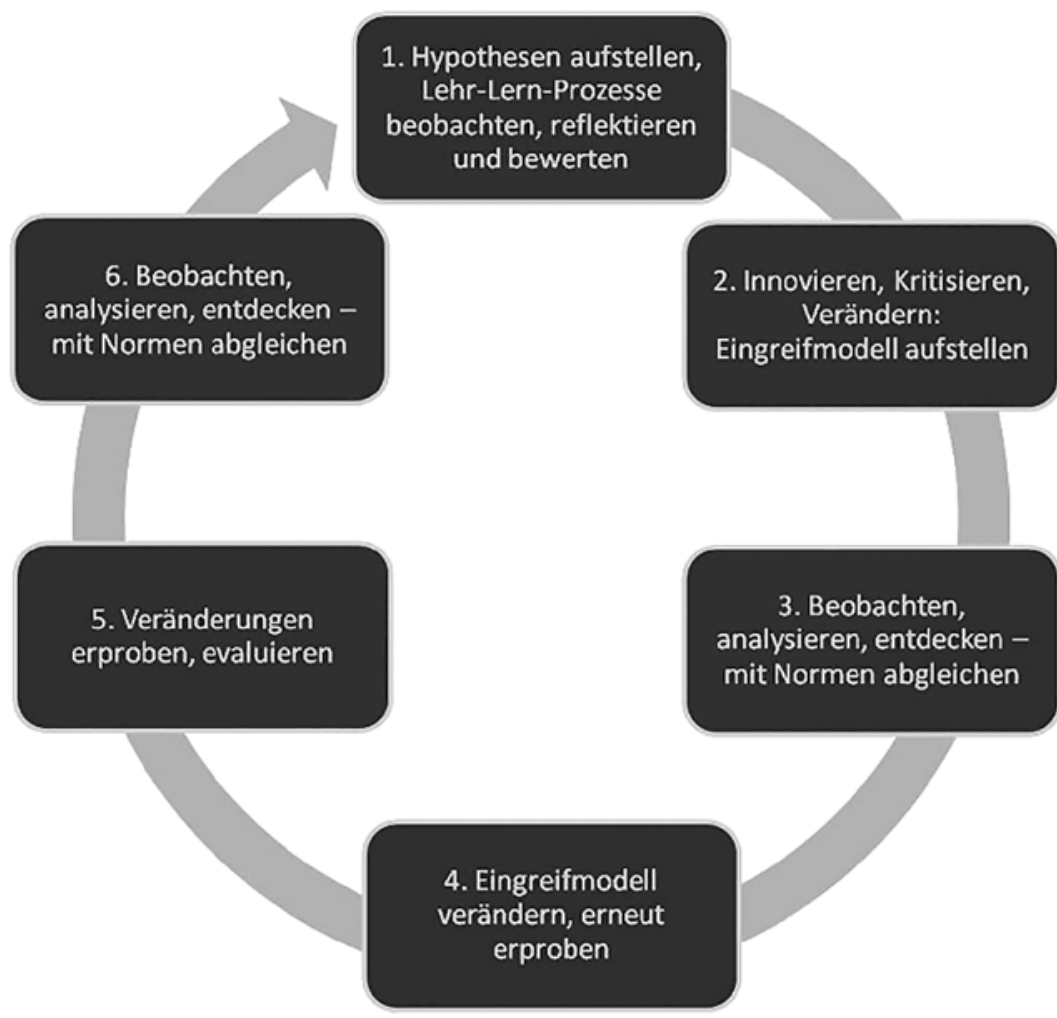


Dieses Prozessmodell ermöglicht eine immer genauere Hypothesenüberprüfung und damit die Ausschärfung bestimmter Konstrukte, z. B. die Operationalisierung von Textkohärenz als unabhängige Variable des Leseverständnisses unter möglichst gleich bleibender ökologischer Validität. So haben wir im Bochum-Wuppertaler Forschungsprojekt zu den textseitigen Determinanten des Leseverständnisses Kohärenz zuerst als morphologische bzw. als textuelle Kategorie aufgefasst. D. h. Kohärenz wird in dieser Konzeption z. B. durch pronominale Verweise an der Textoberfläche hergestellt. Als sich diese Konzeption in der Testung des Leseverständnisses der Schüler nicht signifikant bewährte, haben wir Kohärenz auch als temporale Kategorie aufgefasst. Wir haben Beispieltexte, Testfragen und Auswertungsdesigns damit auch nach den Gesichtspunkten von Vor- und Nachzeitigkeit verändert. Dies bedeutet: Das Leseverständnis der Schüler verändert sich kaum, wenn Texte nur auf der morphologischen und textlinguistischen Ebene verändert werden. Daher bestand Anlass zur Überprüfung der Hypothese, wonach das Leseverständnis entscheidend vom Verständnis der temporalen Vor-, Gleich- und Nachzeitigkeit abhängt. ${ }^{8}$

Die empirisch herbeigeführten Entscheidungen führen immer auch zu einer Veränderung von (schulischen) Lehr-Lern-Prozessen, zu ihrer Implementation und ihrer Innovation. Wie schon am Beispiel der Handlungsfelder erläutert, ist dies nur zum Teil eine wissenschaftliche, sondern mehr noch eine bildungspolitische Aufgabe. Bei der Veränderung geht es darum, wissenschaftliche Erkenntnisse z. B. über die Erreichung oder die Verbesserung von Lesekompetenz zunächst in entsprechende Übungs- und Trainingsprogramme und -szenarien zu übersetzen, ihrerseits zu erproben und dann zu verbessern. Dabei gilt es, institutionelle und persönliche Hürden zu überwinden, um das schülerische Lernen zu verbessern. Unmittelbar umsetzen kann Deutschdidaktik neuere Forschungsergebnisse in der akademischen Lehrerbildung. Schließlich können und sollen Vertreter der Deutschdidaktik sich in der Öffentlichkeit äußern und mehr als bisher Öffentlichkeitsarbeit leisten.

\subsection{Deutschdidaktik als Synthese von Kultur- und Vermittlungswissenschaft}

Man kann beide Konzeptionen von Deutschdidaktik, wie sie bisher erläutert wurden, als gegenseitigen Einschluss, als spannungsreiche Gegenüberstellung oder am besten als Waage auffassen:

8 Vgl. Kröger-Bidlo/Rupp 2013; Rothstein et al. 2014. 
Abb. 4: Waage als Ausgleich zwischen den Polen Vermittlungs- und Kulturwissenschaft

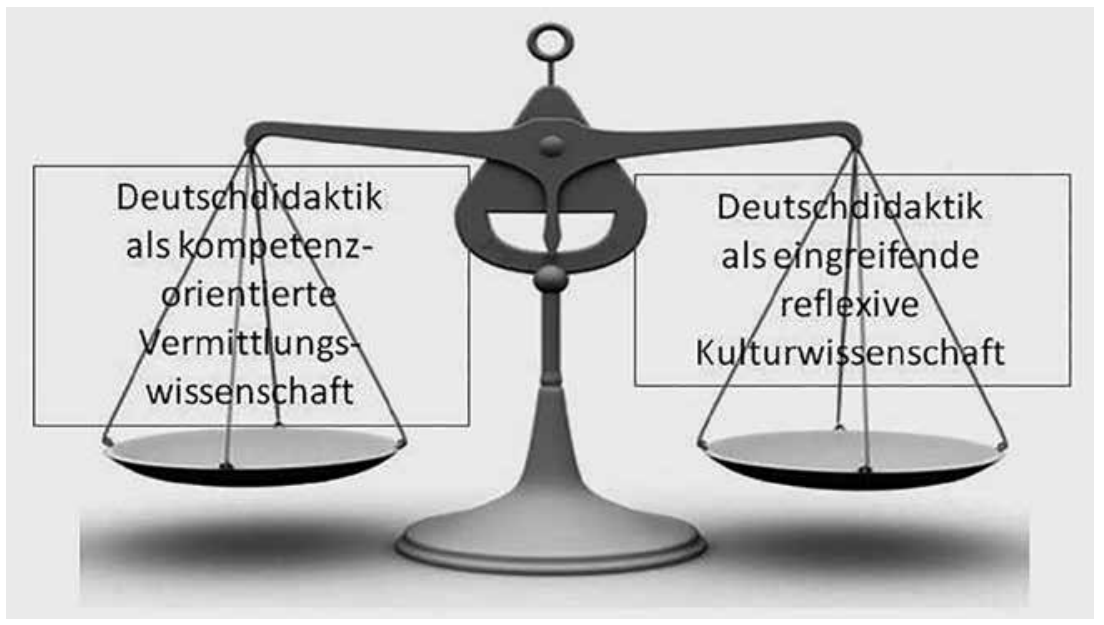

Als kompetenzorientierte Vermittlungswissenschaft formuliert Deutschdidaktik Schüler- und Lehrerkompetenzen für die Lernbereiche des Deutschunterrichts und erprobt Strategien zu ihrer Vermittlung. Als eingreifende reflexive Kulturwissenschaft ist sie nicht nur dem Paradigma der Kompetenzorientierung, sondern eben dem weiteren Paradigma der Kultur verpflichtet, die der „Entfaltung des Individuums dienen“ soll. ${ }^{9}$ Beide Pole stehen in Spannung zueinander, insofern von Seiten der Kulturwissenschaft die Kompetenzorientierung oftmals stark kritisiert wird. Aber Deutschdidaktik sollte als flexible Theorie-PraxisWissenschaft sowohl das Kompetenzparadigma in seiner Leistungsfähigkeit für die Transparenz, Beschreibbarkeit und Optimierung von Lehr-Lern-Prozessen nutzen wie auch den kulturwissenschaftlich weiten, freien Blick auf Deutschunterricht und Schule einnehmen können, der praxeologische Verengungen und Kurzschlüsse vermeiden hilft. Die kulturwissenschaftliche Blickrichtung weitet das Gesichtsfeld aus auf die Vielzahl von sprachlich-literarisch-medialen Lernphänomenen, die dann in vermittlungswissenschaftlicher Engführung bearbeitet werden.

9 Kepser 2013, S. 59; Ingendahl 1991, S. 268. 


\section{Zwei Denkrahmen deutschdidaktischer Entwicklung: das Top Down-Modell und das Modell der symbiotischen Entwicklung}

Bei den bisherigen Ausführungen lag der Fokus rückbezüglich auf der Deutschdidaktik als Disziplin, die sich auf diverse Handlungsfelder bezieht und auf diese einwirkt. Dabei war deutlich geworden, dass es der Deutschdidaktik als Handlungswissenschaft elementar darum geht, durch Implementationen Innovationen und Veränderungen anzustoßen. Gerade unter identitätstheoretischen Gesichtspunkten ist dieser Wechselbezug mit dem sozialen Feld des Unterrichts und letztlich der Gesellschaft elementar wichtig. Die Identität des Faches hängt nämlich nicht nur davon ab, was das Fach selbst an eigenem Profil entwickelt, sondern auch davon, wie dieses wahrgenommen und rückgespiegelt wird.

Das bisher dominante Modell für Implementationen, Innovationen und Veränderungen war und ist das sog. Top Down-Modell, mit dessen Hilfe die durch empirische Untersuchungen ermittelten plausiblen Lehr- und Lernprozesse im Rahmen der Kompetenzorientierung zur allfälligen Realisierung an die Praxis weitergegeben werden. Dieses Modell wird hier aufgegriffen und im Folgenden zu einem „Organisationskompass“ für die gesamte Disziplin ausgebaut.

Allerdings steht das Top Down-Modell nicht unumstritten da. Es sieht die Lehrpersonen lediglich als ausführende AgentInnen. Aufgrund der innerhalb der Deutschdidaktik mittlerweile etablierten Lehrerprofessionalitätsforschung werden Lehrpersonen und ihr professionsbezogenes Wissen nicht nur als Black Box aufgefasst. Im Rahmen der erziehungswissenschaftlichen Lehrerforschung wird ein sog. symbiotisches Entwicklungsmodell diskutiert und auch im Bereich des Deutschunterrichts angewendet: dass didaktische Innovationen mit den Lehrpersonen als gleichberechtigten ExpertInnen zusammen vor Ort konzipiert und durchgeführt werden. ${ }^{10}$ Dieses Modell wird dem Top Down-Modell an die Seite gestellt.

10 Vgl. zur deutschdidaktischen Lehrerforschung u. a. Bräuer/Winkler 2012; zum symbiotischen Entwicklungsmodell im Folgenden vgl. Gräsel/Parchmann 2004. 


\subsection{Der Organisationskompass als Ausprägung des Top Down-Modells deutschdidaktischer Entwicklung}

Ein Organisationskompass gibt eine inhaltliche Wegrichtung und eine klare Handlungsanleitung vor. Die Idee des Organisationskompasses stammt aus der Betriebspsychologie und dem Management-Coaching. Diese Bereiche decken sich auf den ersten Blick nicht mit dem einer Wissenschaftsgemeinschaft. Die Analogiebildung zeigt aber auf, dass man sich am Organisationskompass orientieren könnte, um eigene Ziele wirkungsvoll umzusetzen.

Interessanterweise wird in der Geschichtswissenschaft der Terminus des Denkrahmens in diesem Sinn verstanden. Cornel Zwierlein erläutert mit Bezug auf den Denkrahmen die Entstehung und die Durchsetzung von Geltungsansprüchen im 16. Jahrhundert. Sein Beispiel ist die Denkschrift des Herzogs Carlo Emanuele I von Savoyen mit dem Titel „Discorso, in dem gezeigt wird, dass die Kurfürsten des Reiches jemand anderes als das Haus Österreich erwählen müssen [...] und dass diese Wahl auf das Haus Savoyen fallen müsse“ (2006, S. 13):

Texte von der Art der eingangs genannten Denkschrift sind gleichsam die Entscheidungsfindung selbst: Sie zwingen oder sollen jedenfalls das Denken des Lesenden mit hineinzwingen in ein Durchlaufen eines Weges, der über viele Stationen und Verzweigungen vom Jetzt in ein als möglich konstruiertes Demnächst führt. Die Entscheidung für einen solchen Weg soll das zukünftige Handeln nun als Umsetzung des Planes festlegen. [...] Ein solcher Plan, eine solche Denkschrift, ein solches Gutachten oder Memorial heißt 1618 in Italien offensichtlich ,Discorso. Die Ausgangsprämisse unserer Untersuchung ist die, dass mit diesem Wort eine Methode der, Welt-Bearbeitung', ein Denkrahmen auf den Begriff gebracht wird. (ebd., S. 17)

Man könnte nunmehr den Organisationskompass als moderne Ausformung bzw. als Visualisierung einer solchen Denkschrift verstehen. Der in der Mitte stehende inhaltlich zu definierende Zweck kann nur durch Führung, durch die Geltung von Normen und Leitwerten erreicht werden. Dieser Zweck wird dann nochmals als Handlungsziel („Großes Ziel“) ausbuchstabiert. Mit dem Pol der Gemeinschaft wird deutlich gemacht, dass sich das große Ziel nur im sozialen Zusammenhang von Forschungsgemeinschaften oder im Kontext einer Lerngruppe erreichen lässt. Die „befreienden Strukturen“ heben auf die institutionellen Strukturen ab, die durch ,alternative Strukturen 'überwunden werden können. 
Abb. 5: Organisationskompass als Modell für Organisationen ${ }^{11}$

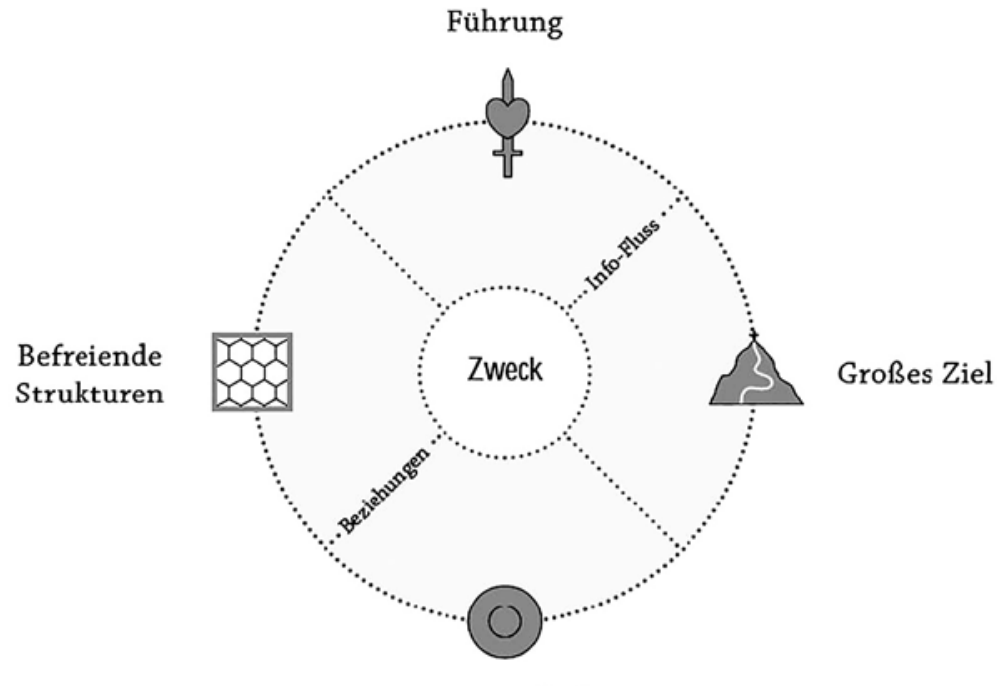

Gemeinschaft

Auch die Deutschdidaktik benötigt einen spezifisch zugeschnittenen Organisationskompass, um deutschdidaktische Ziele zu bestimmen und zu erreichen. Die sich bei der Erreichung dieser Ziele zeigenden Schwierigkeiten sind wissenschaftlicher und außerwissenschaftlicher Natur: Schwierigkeiten der Validierung theoretischer Konstrukte wie z. B. der Textschwierigkeit (vgl. das Prozessmodell realwissenschaftlicher Forschung), Schwierigkeiten der Diagnose, der Förderung und der Implementierung und schließlich Schwierigkeiten der Anerkennung von Forschungsleistungen in unserer wissenschaftlichen Community (Verfügung über Rede- und Publikationsmöglichkeiten, Gewährung von Forschungsaufträgen und von Ressourcen an Geld und Stellen sowie durch die Zuerkennung von Auszeichnungen und Preisen etc.). Die Anerkennung von Forschungsleistungen sieht sich wirkungsmächtigen Strukturen gegenüber. Sie lassen die Ausbildung einer eigenen Corporate Identity angeraten sein. Diese weist Ähnlichkeiten mit der in Unternehmen auf, insofern sie wirtschaftlich, erfolgsorientiert arbeiten müssen. Insofern dient der Organisationskompass der Verdeutlichung dieser Strukturähnlichkeit und zur Verbesserung der eigenen Performance als LehrerbildnerInnen und ForscherInnen: 
Abb. 6: Organisationskompass für die Deutschdidaktik

Denkrahmen als Organisationskompass

$\mathrm{N}$

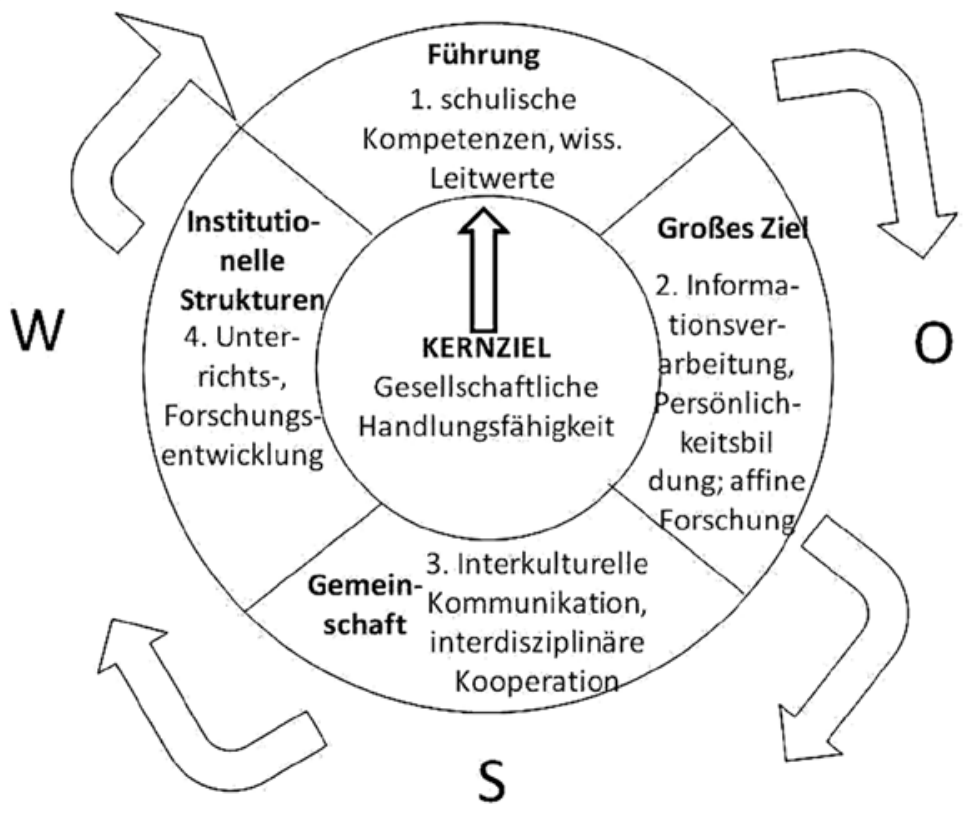

In der Mitte steht das Kernziel, dem sich die Deutschdidaktik als Wissenschaft verschreibt bzw. verschreiben sollte. Dies ist zum einen die Suche nach Wahrheit:

Für den Erhalt des Wissenschaftssystems ist eine zentrale Kodierung zwingend, die Differenz von „wahr“ und „unwahr“. (Kämper van-den-Boogaart 2007, S. 78)

Im Zeichen der Empirie-Orientierung wird das Wahrheitsgebot durch die Bewährung in der Praxis operationalisiert. Dies ist der unhintergehbare Prüfstein für theoretische Modellentwürfe. Es ist zentral für uns DeutschdidaktikerInnen zu wissen, welche Gegenstände, welche Verfahren und welche Vermittlungsansätze erfolgversprechend sind und sich empirisch behauptet haben.

Zum anderen sollte dieser quasi technisch-praktische Wahrheitsbegriff durch einen holistisch-ganzheitlichen Wahrheitsbegriff ergänzt werden. Die Wahrheit im Bereich der Deutschdidaktik besteht in der Bildungsfähigkeit der Schüler durch die Lerngegenstände und -inhalte. Dieser umfassenden Wahrheit dienen die vielen Einzelerkenntnisse aus den drei Ecken des Didaktischen Dreiecks. Diese Wahrheit kann man im Rückgriff auf das konzeptionelle Ergebnis des Schwer- 
punktprogramms „Lesesozialisation in der Mediengesellschaft“ zusammenfassen als Ziel gesellschaftlicher Handlungsfähigkeit aller SchülerInnen ${ }^{12}$ Deutschdidaktik ist somit Anwältin der SchülerInnen „gegen die Mächte.$^{13}$ Dieses Ziel determiniert alle übrigen Bereiche.

Der Norden des Organisationskompasses bezeichnet mit der Führung die obersten Werte und Normen der wissenschaftlichen Forschung und der alltäglichen Vermittlungspraxis. Hier sind u. a. die Bildungsnormen aufgeführt, die in Kompetenzen operationalisiert werden. Hierzu gehören aber auch die wissenschaftlichen Leitwerte der Wahrheitssuche, der Echtheit und der Freiheitsliebe dazu. ${ }^{14}$ Mit Führung ist die Repräsentanz dieser Werte durch vorbildhafte ForscherInnen auch in der Öffentlichkeit gemeint. Konkret denke ich hier an SprecherInnen von Schwerpunktprogrammen und bedeutsame Forscherpersönlichkeiten, die unsere Disziplin nachhaltig geprägt haben.

Im Osten steht das nächste große zu erreichende Ziel, welches der Forschung und der Praxis der Deutschdidaktik aufgegeben ist und welches das Kernziel in der Mitte genauer beschreibt. Im Rückgriff auf die Ergebnisse der großen Schulleistungsstudien liegt dieses nächste große zu erreichende Ziel einerseits in der Verbesserung der Sprach- und der Medienverarbeitung, andererseits in der allseitigen, freiheitlichen personalen Entfaltung, auf die die Schüler ein Anrecht haben. Hier kehren die Waage als Bild und die doppelte Loyalität der Deutschdidaktik wieder.

Während im Norden einzelne Führungspersönlichkeiten herausgehobene Werte vorzeigen, geht es im Süden um die Gemeinschaft, also um den Aspekt der sozialen Verbreitung von Bildungsideen und um die Praxis der Kooperation in Gruppen. Für die Schule ist hier die Leitidee sprachlicher und interkultureller Kommunikation zielführend, für die Wissenschaft die Kooperation in interdisziplinären, internationalen Forschungsverbünden. Norden und Süden sind aufeinander angewiesen, wenn große Konzepte auch sozial breit umgesetzt werden sollen. Die die beiden Pole verbindende Nord-Süd-Achse stellt den menschlichen Faktor des Modells dar.

Der Westen des Organisationskompasses bezeichnet all jene Gegebenheiten, die zur Erreichung des nächsten großen Ziels verbessert und optimiert werden

12 Vgl. Groeben/Hurrelmann 2004. In der Erläuterung dieses zentralen Leitbegriffs wird deutlich, dass es sich nicht um blinden Aktionismus handelt, sondern um ein Handeln, das sich aus theoretischer Reflexion und praktischer Urteilskraft herleitet.

13 Vgl. Kreft 2014, bes. S. 134 ff.: Kreft meint mit „Mächten“ alle Formen der den Einzelnen unterdrückenden Herrschaftsansprüche.

14 Vgl. Lepenies 1997. 
sollten. Dies sind die Strukturen der didaktischen Planung sowie der Klassenund Unterrichtsführung im schulischen Bereich und die institutionellen disziplinären Strukturen der Lehrerbildung und der Forschungsentwicklung im Bereich der Deutschdidaktik. Hier ist eine Fülle von Vorschlägen denkbar, z. B. auf der Ebene der didaktischen Planung die Nutzung verschiedener Lernkanäle oder die Praktizierung effizienter Unterrichtsführung. Im Bereich der Forschungsentwicklung erwähne ich als Erfolgsmodell das Nachwuchsnetzwerk Deutschdidaktik als langjährige Institution der Forschungsförderung und -ermutigung. Zusammengefasst ist der Westen das Spielfeld, auf dem letztlich durch die Beseitigung hinderlicher Barrieren Energien und Potentiale freigesetzt werden, um das nächste große Ziel zu erreichen. So stellt die Ost-West-Achse den sachlichen Faktor des Modells dar.

Zusammengefasst dient das Top Down-Modell dazu, empirisch erwiesene effizienzsteigernde Erkenntnisse optimal an die Praxis weiterzugeben. Dabei ist das kompetenzorientierte Paradigma leitend. Außerdem eignet sich das Top Down-Modell dafür, die Deutschdidaktik als Disziplin nach vorne zu bringen und mehr Corporate Identity zu erzeugen. Trotz all dieser Vorzüge haben ErziehungswissenschaftlerInnen in Zusammenarbeit mit FachdidaktikerInnen des naturwissenschaftlichen Unterrichts Alternativen zum Top Down-Modell entwickelt.

\subsection{Das symbiotische Modell deutschdidaktischer Entwicklung}

Ausgangspunkt der Entwicklung eines symbiotischen Modells deutschdidaktischer Entwicklung ist das schon in den 70er Jahren des vorigen Jahrhunderts immer wieder berichtete Scheitern des Top Down-Modells. Nach Gräsel/Parchmann (2004) mutet es seltsam an, dass in diesem Modell

nicht berücksichtigt wurde, wie schwer Erfahrungswissen, Überzeugungssysteme und Handlungsroutinen zu verändern sind und wie stark der soziale Kontext der Schule auf die Umsetzung einer Innovation Einfluss nimmt [...] Ein zentraler Grund für das Misslingen zahlreicher Reformen kann darin identifiziert werden, dass die Innovationen aufgrund der Trennung zwischen „Konzeptionsebene“ und „Anwendungsebene“ die Bedürfnisse der Praxis häufig zu wenig beachteten [...] (Gräsel/Parchmann 2004, S. 200).

Dieser ganz allgemein für didaktische Implementationen erhobene Befund trifft auch und gerade für die Deutschdidaktik zu, die sich allzu lange mit der Black Box-Vorstellung des Lehrerwissens begnügt und vor allem eine selbstbezügliche, eingeschränkte Blickrichtung auf das komplexe Praxisfeld bewahrt hat. Nach Scherf (2013) müssen die DeutschdidaktikerInnen 
notwendig den Blickwinkel des Fachdidaktikers mit seinen disziplinären Grenzen erweitern [...], um sich den eigenständigen Wissenssystemen der Beforschten unvoreingenommen nähern zu können (Scherf 2013, S. 12; Herv. i. Original). ${ }^{15}$

Man sollte sich somit das Lehrerwissen als Steuerungsmoment des pädagogischen Handelns von Lehrpersonen expliziter vor Augen führen. Der zentrale Befund gerade der deutschdidaktischen Lehrerforschung besteht nun darin, dass das bewusste wissenschaftliche Wissen, über das im Top Down-Modell die Implementation läuft, nur z. T. handlungsleitend ist:

Festzuhalten ist folglich, dass Lehrerhandeln zwar generell als wissensbasiert gilt, dass zahlreiche Kognitionen, auf denen das Handeln fußt, aber als implizit sowie lediglich eingeschränkt bewusst und verbalisierbar angesehen werden. Bewusste Wissensbereiche, z. B. wissenschaftliches Wissen, gelten - positiv formuliert - zumindest nicht als alleinige Wissensgrundlage für Unterrichtshandlungen. (Scherf 2013, S. 33, Herv. i. Original)

Wissenschaftliches Wissen, das die deutschdidaktische Forschung produziert und als Handlungsempfehlung für Innovationen an die Praxis ,weiterreicht', wird also nicht als solches genutzt, sondern u. a. ,produktiv' rezipiert. Die deutschdidaktische Forschung ist also gut beraten sich dafür zu interessieren, wie dieses Wissen verwendet, verändert, transformiert und ,relationiert' wird (vgl. Scherf 2013, S. 36). Andererseits sollte sich die Deutschdidaktik angesichts dieses Tatbestands - neben der weiteren kontinuierlichen Erforschung der deutschdidaktischen Lehrerprofessionalität - zu einem anderen Modell didaktischer Entwicklungsforschung zuwenden, der sog. symbiotischen Implementationsstrategie:

Der Begriff „symbiotische Implementationsstrategie“ soll zum Ausdruck bringen, dass Akteure mit unterschiedlicher Expertise gemeinsam an der Umsetzung pädagogischer Innovationen arbeiten. (Gräsel/Parchmann 2004, S. 205)

Es geht hierbei also nicht um die Umsetzung fertiger Konzeptionen:

Implementation umfasst demnach die Entwicklung, die Verbreitung, die Erprobung und die Revision konkreter Maßnahmen. (Gräsel/Parchmann 2004, S. 206)

Die Konsequenzen dieser Konzeption sind weitreichend, nicht nur für die Konzeptionierung von Implementationsstrategien, sondern für das dahinter stehende Wissenschaftskonzept. Die auf Partizipation ausgerichtete symbiotische Imple-

15 Nach dem von Scherf zitierten Malinowski besteht der „Prüfstein für den echten Arbeiter an der eigentlichen Wissenschaft vom Menschen [...] in der Liebe zur abschließenden Synthese, die durch die Aufnahme und das Begreifen aller Einzelheiten einer Kultur erreicht wird, und noch mehr in der Liebe zur Vielfalt und Unabhängigkeit der verschiedenen Kulturen" (Malinowski 1979, S. 557). 
mentationsstrategie verlangt letztlich von den beteiligten WissenschaftlerInnen, einen Teil ihrer Autonomie und auch ihres Anteils an der Bestimmung der Identität der eigenen Disziplin an die kooperierenden ExpertInnen abzugeben:

Diese Strategien implizieren für Wissenschaftler/-innen eine gänzlich neue Rollendefinition. Sie geben ihre Erkenntnisse nicht unverändert in die Praxis weiter, sondern konstruieren gemeinsam mit anderen Personen neues Wissen. Ihr wissenschaftliches Wissen ist dabei dem Erfahrungswissen von Lehrpersonen und dem Steuerungswissen von Bildungsadministratoren gleichrangig. Durch Kommunikation und Austausch der verschiedenen Perspektiven und „Wissensformen“ werden Maßnahmen entwickelt, umgesetzt, überprüft und weiterentwickelt. (Gräsel/Parchmann 2004, S. 209 f.)

Mindestens so wichtig wie die Umsetzung wissenschaftlicher Erkenntnisse im skizzierten Dialog zwischen TheoretikerInnen und PraktikerInnen sind daher die Etablierung von dauerhaften Kooperationsstrukturen und das Ziel, „die Lernund Organisationskultur an Schulen insgesamt zu verbessern“ (ebd.).

Besonders eindrucksvoll wurde die symbiotische Implementationsstrategie im Bereich der Chemiedidaktik im Projekt „Chemie im Kontext“ (CHiK) umgesetzt:

Die Unterrichtskonzeption Chemie im Kontext kann hier nur kurz skizziert werden [...] Im Zentrum jeder Unterrichtseinheit dieses Unterrichtskonzepts stehen so genannte Kontexte, worunter Anwendungsfelder oder authentische Probleme verstanden werden, auf die das zu erlernende chemische Wissen angewendet werden kann [...]. Die Kontexte können alltagsnah sein (z. B. „Ein Mund voll Chemie“), sie können aber auch gesellschaftlich relevante Fragestellungen oder einen Bezug zur Forschung thematisieren (z. B. „Das Wasserstoffauto als Fahrzeug der Zukunft?"). Anhand der Kontexte formulieren die Schülerinnen und Schüler zunächst (Forschungs-)Fragen, die sie im weiteren Verlauf des Unterrichts selbst untersuchen und mit ihren Ergebnissen beantworten [...] Schließlich wird das Prinzip der Basiskonzepte realisiert, das eine Systematisierung und Dekontextualisierung des erworbenen Wissens darstellt, das insbesondere am Ende der Unterrichtseinheit in der „Vernetzungsphase“ geleistet wird. (Gräsel 2011, S. 91) $)^{16}$

Neben den bisher skizzierten Merkmalen der symbiotischen Implementationsstrategie fallen hier die authentischen Probleme, der Kontext sowie die Basiskonzepte auf, die einen dezidierten fachspezifischen Bezug und damit ein weiteres

16 Für den Deutschunterricht einschlägige Bereiche, in denen das Konzept symbiotischer Implementation erprobt wurde, sind u. a. das Projekt „Förderung der Lesekompetenz in Hauptschulbildungsgängen“ („Lesekorn“, vgl. Gräsel 2011 S. 95 ff.), das Programm „Förderung von Kindern und Jugendlichen mit Migrationshintergrund“ (FörMig) (vgl. Schründer-Lenzen/Mücke 2011) und das Forschungsprojekt „Leseprofi im Leseteam Lesestrategien für die Grundschule“ (vgl. Kirschhock/Munser-Kiefer 2011). 
Bestimmungsmoment dieses Entwicklungsmoments ausmachen. Ähnlich heißt es bei Scherf (2013):

Die Entwicklung empirischer Forschungsmethoden, die sich an relevanten Bezugswissenschaften zu orientieren hat, muss allerdings mit fachdidaktischem Fokus erfolgen, schließlich verfügt die Disziplin über ihre eigenen Zielsetzungen, die aus ihrem fachlichen Gegenstand resultieren. Sie muss daher m. E. ihre Stimme im Konzert der empirischen Bildungsforschung fachbezogen und selbstbewusst vertreten, statt sich an andere Stimmen anzuhängen. (Scherf 2013, S. 441)

\subsection{Top Down` und ,Symbiose` als sich ergänzende Optionen für die Identitätsbestimmung der Disziplin}

Je nach Kontext und Situation wird die Deutschdidaktik in der Zukunft auf die beiden skizzierten Optionen didaktischer, disziplinärer und gesamtgesellschaftlicher Entwicklung zurückgreifen müssen. In Anwendung der MehrebenenAnalyse der Gesellschaft von Esser (1999) unterscheidet man eine Makro-Ebene, auf der bereits gesicherte wissenschaftliche Erkenntnisse nachhaltig realisiert und in der Gesellschaft verankert werden, von einer Meso-Ebene, auf der in institutioneller Perspektive Innovationen symbiotisch entwickelt und umgesetzt werden. Auf der Mikro-Ebene agieren einzelne Individuen, z. B. Deutschlehrkräfte. Aufgrund der zwischen den Ebenen anzusetzenden „Brücken“ und „Übergänge“ ist von einer möglichst produktiven Interaktion zwischen den Ebenen auszugehen. Dies sollte auch für die Lösung der Aufgaben der Deutschdidaktik im 21. Jahrhundert gelten, die z. T. den engeren Rahmen der Wissenschaft überschreiten und die im Folgenden benannt werden.

\section{Aufgaben der deutschdidaktischer Forschung im 21. Jahrhundert}

Zur Bestimmung von Denkrahmen und Denkstilen gehören konkrete programmatische Sätze, durch welche diejenigen Aufgaben bezeichnet werden, die sich der Deutschdidaktik im 21. Jahrhundert stellen. Sie bezeichnen zusätzlich zu dem Denk- einen Handlungsrahmen sowie konkrete Optionen, die die jungen ForscherInnen nutzen können. Zusammengefasst bestehen sie in den fünf Punkten der Interdisziplinarität, der Internationalität, der Vernetzung mit der Praxis, der Verbesserung der Corporate Identity und der Öffentlichkeitsarbeit. 


\subsection{Interdisziplinarität als unhintergehbare Konstellation - als Korrektiv sich einspielender Konsensgemeinschaften}

Die Forschungsentwicklung der letzten 25 Jahre hat der Deutschdidaktik im Bereich drittmittelgeförderter Projekte einen erheblichen Aufwind beschert. Der erreichte Standard meist interdisziplinärer, empirisch ausgerichteter Vorhaben sollte nicht mehr verlassen werden. Interdisziplinäre Konstellationen fungieren dabei als Prüfstein und als Korrektiv eingespielter Konsensgemeinschaften, als Herausforderung zur Operationalisierung oder zur Explizierung eigener Hypothesen.

\subsection{Internationalität als Überwindung des Kirchturmdenkens und -handelns}

Seit dem Forschungsverbund des International Mothertongue Education Network (IMEN), seit PISA, IGLU und DESI ist international ausgerichtete, vergleichende oder kooperative Forschung der zweite Standard deutschdidaktischer Forschung. Diese Internationalität beginnt bei den deutschsprachigen Ländern und reicht über Europa in alle Länder der Ersten Welt. Sie schließt mit China und Japan besonders die asiatischen fernöstlichen Länder ein, während Südamerika, Afrika und Australien noch weitgehend blinde Flecken darstellen. Neben den u. a. durch die Aktivitäten des Europarats geförderten europäischen Verbindungen sind besonders die Querverbindungen zu amerikanischen Lese- und SchreibforscherInnen wie z. B. zu Donna Alverman hervorzuheben. Aber noch immer beteiligen sich an solchen Kontakten relativ wenige KollegInnen und publizieren zu wenige in internationalen Zeitschriften.

Allerdings darf die internationale Ausrichtung auch nicht übertrieben oder als Selbstzweck verfolgt werden. Gerade im Lichte symbiotischer Implementationsstrategien wird der Fachbezug auf eine im engeren Sinn muttersprachliche Deutschdidaktik mit spezifischen Interessen und autochthonen Gegenstandsbezügen genauso wichtig wie die Repräsentanz auf internationaler Ebene.

\subsection{Vernetzung mit Schulen als Überwindung der 2-Phasen- Didaktiken, des Theorie-Praxis-Schismas - Best Practice, Laborschulen, Schülerlabore an Unis}

Die nötige Vernetzung mit der Schulpraxis als Teil der Forschungsausrichtung und der Lehrerbildung war einer der Hauptpunkte der Kritik, die in einem Gutachten des Stifterverbandes an den Fachdidaktiken geübt worden ist. ${ }^{17}$ In der Folge sind Projekte gefördert worden, die genau diese Ausrichtung unterstützen. 
Wie zwischen Kompetenz- und Kulturorientierung sollte auch zwischen Theorie und Praxis ein angemessener Ausgleich hergestellt werden. Es mangelt ja nicht an Unterrichtsvorschlägen und Stundenblättern, allerdings mangelt es an Praxis-Tipps, die aus fundierter Forschung hervorgehen. Deswegen sollte die forschungsorientierte Deutschdidaktik wenigstens zu einem Teil die Praxis direkt mitbestimmen und hier angestammtes Terrain zurückgewinnen. Handlungsfelder sind hier die Kooperation mit der Referendarausbildung (bis hin zur Mitwirkung bei der 2. Staatsprüfung) ${ }^{18}$, die Ausweisung von Best Practice-Schulen und die Einrichtung von Schülerlaboren an den eigenen Hochschulen.

\subsection{Corporate Identitiy im Fach - Vorbild MINT-Fächer}

Während Interdisziplinarität und Internationalität die Forschung und die Praxisorientierung schwerpunktmäßig die Lehrerbildung betreffen, geht es bei der Corporate Identity um die interne Kommunikation im Fach. Hier kann auf den Westpol des Organisationskompasses und auf die institutionellen Strukturen verwiesen werden. Auch hier sollte die Deutschdidaktik von anderen Fachkulturen lernen: von der Publikationspraxis als Forschergruppe, von der direkten kollegialen Kooperation unter vielen KollegInnen der MINT-Fächer, von der kollegialen Unterstützungspraxis bei Forschungsanträgen etc. Bei meinen Kontakten mit KollegInnen aus anderen Fachdidaktiken und der Lehr-Lern-Forschung habe ich eine Reihe solcher selbstverständlicher Praktiken und Rituale kennen und schätzen gelernt und selbst in meinem Bereich zu integrieren versucht.

\subsection{Repräsentanz der Deutschdidaktik in der Öffentlichkeit}

Während die Corporate Identity im Wesentlichen die Kommunikation nach innen betrifft, geht es bei der Repräsentanz der Deutschdidaktik in der Öffentlichkeit um die Kommunikation nach außen, um die verständliche Vermittlung von Forschungsergebnissen in den Medien an die Öffentlichkeit, die diese Forschung ja finanziert. Und es geht auch um die Besetzung entsprechender Kommunikationsplätze in Zeitungen und Medien. Es geht um die Erweiterung des Publikums: Das Publikum der Wissenschaft soll und braucht nicht immer nur die Wissenschaft selbst sein, also die maximal ca. 1000 Mitglieder unserer Community. Nach Terhart stellt sich die Aufgabe der

Darstellung und Vermittlung von Wissenschaften in der Öffentlichkeit, in Museen, in autonomen Lernzentren, via Lernplattformen und Wissenschaftsparks, in Schülerlaboren etc. [...] Die Vermittlung ihrer selbst in ihre verschiedenen Umwelten hinein gehört eigentlich zu den genuinen, inneren Aufgaben von Disziplinen [...] (Terhart 2011, S. 252).

18 Vgl. Gerner 2014, S. 190. 
Es gibt eine lange Tradition und Präsenz von deutschdidaktischen KollegInnen in Hochschulleitungspositionen, von denen aus diese KollegInnen auch weithin sichtbar waren und sind - aber diese Positionen könnten ausgebaut werden. Wenigstens das eine oder andere Mal könnten ForscherInnen auch die Kommunikator-Rolle einnehmen und z.B. sich an der Schulzukunftsdebatte beteiligen, die Richard David Precht mit seinem Bestseller „Anna, die Schule und der liebe Gott" (2015) angestoßen hat. Oder sie könnten selbst initiativ werden und Themen vorgeben. Die letzte bedeutende Initiative in dieser Richtung ist seinerzeit von Marcel Reich-Ranicki, also von den Medien in Richtung Wissenschaft ausgegangen: Reich-Ranicki vertraute darauf, dass LiteraturwissenschaftlerInnen Kritiker sein und Rezensionen schreiben könnten. Müssen wir uns am Ende bitten lassen? Die fachkundige und formatgerechte Aufarbeitung von Best Practice-Beispielen wäre es doch wert, in die Öffentlichkeit zu gehen. Und auch unabhängig davon könnten deutschdidaktische Intellektuelle stärker in der Öffentlichkeit auftreten und die Sache des Lernens und der Bildung vertreten, dies müssten nicht nur immer dieselben unvermeidlichen Gumbrecht, Safranski oder Sloterdijk sein. Eine unmittelbar zu realisierende Maßnahme ist die Einrichtung einer Expertenvermittlung für JournalistInnen auf der Seite des Symposions Deutschdidaktik nach dem Vorbild der Deutschen Gesellschaft für Psychologie. ${ }^{19}$

\section{Fazit}

Der Denkrahmen der Deutschdidaktik besteht einerseits in ihrer Bestimmung als eingreifende Kulturwissenschaft. Hier kann aufgrund einer weiten Suchrichtung ,alles' zum Thema der Rekonstruktion und der Konstruktion werden. Andererseits ist Deutschdidaktik empirie-orientierte experimentelle Wissenschaft, die möglichst viele Kompetenzmodellierungen prüft und validiert. Auf dieser Ebene ist Deutschdidaktik mit anderen Fachdidaktiken und mit der internationalen Forschung kompatibel. Hier besteht immer noch ein erheblicher Nachholbedarf nicht zuletzt für die Literaturdidaktik:

Eine Deutschdidaktik, die sich als Wissenschaft versteht und als Wissenschaft ernst genommen werden will, muss überprüfte Theorien und abgesichertes Wissen generieren. Als Wissenschaftler müssen wir schlicht wissen, ob das, was wir theoretisch modellieren und als praxistauglich empfehlen, tatsächlich die erhofften Wirkungen zeitigt. Diesem Erfordernis kann nur auf empirischer Basis entsprochen werden. (Frederking 2014, S. 110)

19 Vgl. http://www.dgps.de/index.php?id=46. Dankenswerter Hinweis von N. Groeben, Heidelberg. 


\section{Literatur}

Baum, Michael/Bönninghausen, Marion (Hrsg.) (2010): Kulturtheoretische Kontexte für die Literaturdidaktik. Baltmannsweiler: Schneider Verlag Hohengehren.

Betz, Anica/Koch, Florian/Reinsch, Maximilian/Vorberg, Julika (2013): „Wandel durch Kultur" - Anregungen für einen zeitgemäßen Deutschunterricht im Ruhrgebiet. In: Frickel, Daniela A. /Boelmann, Jan M. (Hrsg.): Literatur, Lesen, Lernen. Festschrift für Gerhard Rupp. Frankfurt am Main: Peter Lang Edition, S. $17-42$.

Bräuer, Christoph/Winkler, Iris (2012): Aktuelle Forschung zu Deutschlehrkräften. Ein Überblick. In: Didaktik Deutsch. 17. Jg., H. 33, S. 74-91.

Erikson, Erik H. (1966): Das Problem der Ich-Identität. In: Erikson, Erik H.: Identität und Lebenszyklus. Drei Aufsätze. Frankfurt: Suhrkamp, S. 123-215.

Esser, Hartmut (1999): Situationslogik und Handeln. Frankfurt/Main: CampusVerl.

Frederking, Volker (2014): Deutschdidaktik als transdisziplinäre, anwendungsund grundlagenorientierte empirische Wissenschaft. In: Mitteilungen des Deutschen Germanistenverbandes. 61 (2), S. 109-119.

Gerner, Volker (2014): Die Didaktikwissenschaft Deutsch und ihre Bezüge zur Bildungswissenschaft / Erziehungswisssenschaft / Pädagogik. In: Frederking, Volker/ Krommer, Axel (Hrsg.): Taschenbuch des Deutschunterrichts. Aktuelle Fragen der Deutschdidaktik. 3 Bände. Baltmannsweiler: Schneider Verlag Hohengehren, S. 177-196.

Gräsel, Cornelia (2011): Die Kooperation von Forschung und LehrerInnen bei der Realisierung didaktischer Innovationen. In: Einsiedler, Wolfgang (Hrsg.): Unterrichtsentwicklung und Didaktische Entwicklungsforschung. Bad Heilbrunn: Klinkhardt, S. 88-105.

Gräsel, Cornelia/Parchmann, Ilka (2004): Implementationsforschung - oder: der steinige Weg, Unterricht zu verändern. In: Unterrichtswissenschaft. 32 (3), S. 196-214.

Groeben, Norbert/Hurrelmann, Bettina (2004): Fazit: Lesen als Schlüsselqualifikation? In: Groeben, Norbert/Hurrelmann, Bettina (Hrsg.): Lesesozialisation in der Mediengesellschaft. Ein Forschungsüberblick. Weinheim: Juventa, S. $440-466$.

Ivo, Hubert (1996): Über den Tag hinaus. Begriff einer allgemeinen Sprachdidaktik. In: Didaktik Deutsch. 1. Jg., H. 1, S. 8-29. 
Kämper-van den Boogaart, Michael (2007): Fachdidaktik und Wissenschaft. In: Kämper-van den Boogaart, Michael (Hrsg.): Deutsch-Didaktik. Leitfaden für die Sekundarstufe I und II. Berlin: Cornelsen-Scriptor, S. 75-94.

Kepser, Matthis (2013): Deutschdidaktik als eingreifende Kulturwissenschaft. Ein Positionierungsversuch im wissenschaftlichen Feld. In: Didaktik Deutsch. 18. Jg., H. 34, S. 52-68.

Kirschhock, Eva-Maria/Munser-Kiefer, Eva-Maria (2011): Didaktische Entwicklungsforschung am Beispiel Lesestrategien - Entwicklung und empirische Überprüfung eines Lesestrategie-Trainings. In: Einsiedler, Wolfgang (Hrsg.): Unterrichtsentwicklung und Didaktische Entwicklungsforschung. Bad Heilbrunn: Klinkhardt, S. 125-143.

Kreft, Jürgen (2014): Der Deutschunterricht zwischen Germanistik und Bezugswissenschaften. In: Frederking, Volker/Krommer, Axel (Hrsg.): Taschenbuch des Deutschunterrichts. Aktuelle Fragen der Deutschdidaktik. 3 Bände. Baltmannsweiler: Schneider Verlag Hohengehren, S. 126-142.

Kröger-Bidlo, Hanna/Rupp, Gerhard (2013): Textkohäsion als Bedingung des Leseverständnisses bei der Verarbeitung expositorischer Texte. In: Didaktik Deutsch. 18. Jg., H. 35, S. 82-101.

Lepenies, Wolf (1997): Benimm und Erkenntnis. Über die Notwendigkeit der Rückkehr der Werte in die Wissenschaften. Die Sozialwissenschaften nach dem Ende der Geschichte. Zwei Vorträge. Frankfurt: Suhrkamp.

Malinowski, Bronislaw (2007): Argonauten des westlichen Pazifik. Ein Bericht über Unternehmungen und Abenteuer der Eingeborenen in den Inselwelten von Melanesisch-Neuguinea. Eschborn bei Frankfurt am Main: Klotz.

Müller-Michaels, Harro (2014): Aufgaben und Diskurse der Didaktik. In: Frederking, Volker/Krommer, Axel (Hrsg.): Taschenbuch des Deutschunterrichts. Aktuelle Fragen der Deutschdidaktik. 3 Bände. Baltmannsweiler: Schneider Verlag Hohengehren, S. 117-126.

Ossner, Jakob (2001): Elemente eines Denkstils für didaktische Entscheidungen. In: Rosebrock, Cornelia/Fix, Martin (Hg.): Tumulte. Deutschdidaktik zwischen den Stühlen. Baltmannsweiler: Schneider Verlag Hohengehren, S. 17-32.

Precht, Richard David (2015): Anna, die Schule und der liebe Gott. Der Verrat des Bildungssystems an unseren Kindern. München: Goldmann.

Rothstein, Björn/Kröger-Bidlo, Hanna/Gräsel, Cornelia/Rupp, Gerhard (2014): Überlegungen zur Messung des Kohäsionsgrads von Texten. In: Linguistische Berichte. (237), S. 37-56.

Rupp, Gerhard (Hg.) (2008): Spessart als Text. Analyse und Aneignung einer Landschaft. Hanau: CoCon-Verl. 
Rupp, Gerhard (2014): Deutschunterricht lehren weltweit. Basiswissen für Master of Education-Studierende und DeutschlehrerInnen. Baltmannsweiler: Schneider Verlag Hohengehren.

Rupp, Gerhard/Bonholt, Helge (2006): Lehr-Lern-Forschung als empirische Lese-/Literaturdidaktik?! In: Groeben, Norbert und Hurrelmann, Bettina (Hrsg.): Empirische Unterrichtsforschung in der Literatur- und Lesedidaktik. Ein Weiterbildungsprogramm. Weinheim: Juventa-Verl., S. 239-253.

Scherf, Daniel (2013): Leseförderung aus Lehrersicht. Eine qualitativ-empirische Untersuchung professionellen Wissens. Wiesbaden: Springer VS.

Schründer-Lenzen, Agi/Mücke, Stephan (2011): Entwicklung und Evaluation eines Sprachförderprogramms - Das Modellprogramm FörMig in Brandenburg. In: Wolfgang Einsiedler (Hrsg.): Unterrichtsentwicklung und Didaktische Entwicklungsforschung. Bad Heilbrunn: Klinkhardt, S. 105-124.

Terhart, Ewald (2011): Zur Situation der Fachdidaktiken aus der Sicht der Erziehungswissenschaft: konzeptionelle Probleme, institutionelle Bedingungen, notwendige Perspektiven. In: Bayrhuber, Horst et al. (Hrsg.): Empirische Fundierung in den Fachdidaktiken. Münster u. a.: Waxmann, S. 241-257.

Ulrich, Peter/Hill, Wilhelm (1976): Wissenschaftstheoretische Grundlagen der Betriebswirtschaftslehre. In: Wirtschaftswissenschaftliches Studium: Zeitschrift für Ausbildung und Hochschulkontakt. 5 (7 + 8), S. 304-309, 345-350.

Weyand, Birgit (Hrsg.) (2012): Auf unsere Lehrerinnen und Lehrer kommt es an. Geeignete Lehrer/-innen gewinnen, (aus-)bilden und fördern. Essen: Ed. Stifterverb. für die Dt. Wissenschaft.

Zwierlein, Cornel (2006): Discorso und Lex Dei. Die Entstehung neuer Denkrahmen im 16. Jahrhundert und die Wahrnehmung der französischen Religionskriege in Italien und Deutschland. Göttingen: Vandenhoeck \& Ruprecht.

Zur Bonsen, Matthias (2010): Der Organisationskompass. Ein neuer Denkrahmen für lebendige Unternehmen. In: Galuska, Joachim und Spiegel, Peter (Hrsg.): Die Kunst des Wirtschaftens. Bielefeld: Kamphausen, S. 183-199. 
Harro Müller-Michaels

\title{
Denken in Ellipsen - Didaktik im Spannungsfeld von Bildung und Wissenschaften
}

\section{Elliptical Thinking - Didactics as interplay between education and sciences}

\begin{abstract}
The didactics mindset can only be multi-dimensional. Different disciplines apply, depending on the research project, which may include discussing the aims of education, justifying curricular content, or performance testing. The research dimensions are described using ellipses with two intersections each. Various discussion formats ranging between explanation and normativity address the differing leading interests behind scientific works and are explained with the aid of examples.
\end{abstract}

\section{Didaktische Forschung: Drei Ebenen}

Eigentlich ist der Gegenstand jeder Primärsprachen Didaktik leicht zu definieren: als Theorie des Unterrichts in Sprache und Kultur der Muttersprache für alle Altersstufen im primären und sekundären Bildungssektor. Aber mit der unspezifischen Festlegung beginnen die Probleme: Welche Ziele verfolgen Schule und Unterricht?, Was soll gelernt werden?, Welche Methoden mit welchen Medien sollen geübt werden?, Wie sind die konkreten individuellen, sozialen und politischen Bedingungen in Schule und Klasse?, Wie werden Leistungen der Schülerinnen und Schüler sowie des Unterrichts bewertet? Sollen die Standards überall und für alle gleich sein?, Wie sind die Lehrerinnen und Lehrer ausgebildet? Aus dem vergleichsweise kleinen Aufgabenfeld erwächst eine Fülle von Problemen, die bearbeitet werden müssen. Lösungen bietet die Didaktik in der Weise an, dass sie aus der Praxis heraus die Fragen bündelt, die Problemstellungen mit den einschlägigen Bezugswissenschaften diskutiert, Modelle entwirft und an die Praxis zur Prüfung zurückgibt. Didaktik wird auf diese Weise zum innovativen Korrektiv für die Praxis.

Angesichts der Fülle von wiederkehrenden und neuen Problemen erscheint es sinnvoll, die Didaktik weniger als eine Wissenschaft mit einem eigenen, klar definierten Sachgebiet, sondern als ein Bündel von Fragerichtungen zu verstehen. Mit jeder Frage landen wir in anderen Disziplinen: bei den Bildungs- 
wissenschaften (Ziele), Fachwissenschaften (Inhalte), der Psychologie (Methoden und Prüfungen), der Soziologie (Herkunft, Differenzierung) oder Philosophie (Erkenntnistheorie, Ethik). Wenn Didaktik nicht zur Rezeptologie verkümmern will, die aus einem aus bewährter Erfahrung oder administrativer Anordnung geleiteten Denkhorizont Handlungsanregungen gibt, bleibt sie auf die Diskussion mit den Bezugswissenschaften angewiesen, die neue empirische Einsichten, Theorieentwürfe, Methodenkonzepte oder Modelle für reflektiertes Handeln bereitstellen. Mit Blick auf diese Doppelstruktur wissenschaftlicher Arbeit bietet sich das Bild der Ellipse mit ihren zwei Brennpunkten (entgegen all den modischen einsinnigen Zugriffen) an, wenn wir über einen Denkrahmen der Didaktik nachdenken, weil auf diese Weise deutlich wird, wie leitende Fragen nur in interdisziplinären Diskursen komplexe und überzeugende Antworten finden können. Von den Diskursen profitieren nicht nur die Didaktiken, sondern auch die befragten Wissenschaften, insofern sie allgemeine Einsichten auf konkrete und bedeutende Fälle anwenden können, wenn z. B. die Psychologie die Kompetenzen in den Muttersprachen zu dem zentralen Gegenstand der Lernstandserhebungen macht oder die Literaturwissenschaften das Feld von literarischer Wertung und Kanonbildung besetzen. Pluralität der Zugriffe ist die angemessene Antwort auf die Frage nach dem Ort der Didaktik im Spektrum der Wissenschaften. Je nach der leitenden Problemstellung sollen drei Ebenen dieser Perspektiven des Fragens und Antwortens unterschieden werden:

- Ebene 1: Wissenschaften und ihre Leistung für die Bildung

- Ebene 2: Theorie und Praxis: Handlungsforschung im Schulfeld

- Ebene 3: Wissenschaftstheorie: Grundlagen und Anwendung

Auf jeder dieser Ebenen treten originäre didaktische Fragestellungen in produktive Verbindung mit einem anderen Brennpunkt, sodass wir von drei ineinander gefügten Ellipsen als Rahmen didaktischer Forschung und Lehre sprechen können.

Denkrahmen sind daher im Kontext variabler Bezugsebenen in der Didaktik sowohl in einem engen, präzisen als auch in einem weiten, generellen Sinne zu verstehen: Einerseits bündeln sie konkrete Fragestellungen zu Allgemeiner Bildung (Ebene 1), Leistungsstandards (Ebene 2) oder zu Qualitativer Unterrichtsforschung (Ebene 3), andererseits umfassen sie das ganze Feld der drei Ellipsen, indem sie ihren Beitrag zur Leistung, Reichweite und Validität der Forschungen in den Angewandten Wissenschaften liefern. ,Denkrahmen ' wird dabei verstanden als Begrenzung (Definition) der Forschungsfelder durch ihre Gegenstände, methodischen Verfahren, leitende Absichten und Rhetorik der Darstellung. 


\subsection{Ebene 1: Wissenschaften und Bildung}

Im Spektrum der Disziplinen der Universitäten begründen die Didaktiken den Blick auf die Leistungen der Wissenschaften für Bildung und Unterricht. Während sich die Fachwissenschaft (z. B. die Germanistik) die Untersuchung der Systematik und Geschichte sowie die Produktion von Editionen, Plausibilisierungen von Interpretationen oder Literarische Wertungen zur Aufgabe setzt, stehen im Brennpunkt der Didaktik Fragen der Bedeutung des Wissens und Könnens für die Öffentlichkeit und die Bildung der nächsten Generation. Der Vorwurf, gegen den sich meine Generation nach der Integration der Pädagogischen Hochschulen in die Universitäten immer wieder wehren musste, dass wir mit der Frage nach dem Nutzen des Wissens und der des methodischen Könnens in Anwendungsfeldern die Zweckfreiheit der Wissenschaften unterlaufen, war mit dem Argument zu entkräften, dass auch didaktische Entscheidungen und Modelle zunächst nach Wertigkeit des Wissens, rationaler Plausibilität der Entwürfe, Angemessenheit der Vorhaben für die Situation ihres Gebrauchs begründet werden müssen. Freiheit der Forschung gilt auch für die Didaktik, sofern sie nicht allein nach Praktikabilität fragt oder sich nicht eilfertig in den Dienst politischer Vorgaben stellt. Eine unvermittelte Übertragung theoretischer Konstrukte in die Praxis ist ebenso wenig reflektiert wie die Übernahme praktischer Rezepte in die Theorie des Unterrichts. Der Hiatus zwischen Theorie und Praxis muss bekräftigt werden, um die je eigenen Gesetze der Handlungs- und Denkebenen im je anderen Kontext produktiv werden zu lassen.

\section{Abb. 1: Bildungswissenschaften}

\section{Bildungswissenschaften}

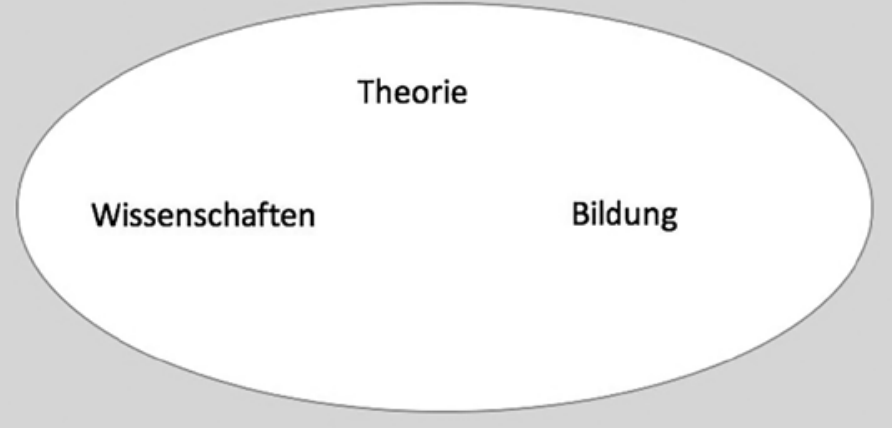


Insofern müsste, wenn er sich nicht von alleine einstellt, der Praxisschock bei jungen Lehrenden ausgelöst werden, um die Reflexion in mindestens zwei Ebenen in Gang zu setzen und zu halten. Die Dissonanzerfahrung gilt auch für den Fall, dass zur Lösung eines praktischen Problems theoretische Modelle recherchiert, diskutiert und erprobt werden müssen.

Die Denkbewegungen der Didaktik als Theorie des Lehrens und Lernens laufen zwischen den Gegenständen von Unterricht und Erziehung, den Bedingungen des Unterrichts in konkreten Fällen und den Zielen des Denkens und Handelns in beide Richtungen, um Analysen und Konstruktionen von Modellen plausibel zu machen. Von den beharrlichen Fragen profitieren auch die Disziplinen im zweiten Brennpunkt, wenn bspw. die Pädagogik mit der Primärsprache ein relevantes Gegenstandsfeld gewinnt und die Germanistik ihren Denkhorizont auf die Wirksamkeit ihrer Gegenstände für die Bildung der nächsten Generation erweitert.

In der Kerndebatte über die Ziele von Unterricht und Erziehung gibt es ein Wechselspiel zwischen den Bildungs- und Kulturwissenschaften. Eine plausible Bestimmung von Bildung stammt von Heinz-Elmar Tenorth, der Allgemeinbildung folgendermaßen definiert hat:

Allgemeine Bildung zu vermitteln, lässt sich als die konkrete (pädagogische) Aufgabe beschreiben, ein Bildungsminimum für alle zu sichern und zugleich die Kultivierung von Lernfähigkeit zu eröffnen. (Tenorth 1994, S. 166)

Mein Vorschlag, mit ähnlicher Verbindung zu den Ideen Humboldts, fügt dem Wissen und Können die wachsende Verantwortung für sich und andere als Ziel der Persönlichkeitsbildung hinzu:

Bildung ist das Vermögen, Wissen, Können und Verantwortung so zu entwickeln, dass daraus das Bedürfnis wird, alle eigenen Möglichkeiten ganz auszuschöpfen, um über sich hinauszuwachsen. (Müller-Michaels 2009, S. 42)

Mit der Diskussion der Ideen von Bildung sowie ihrer Konkretisierung in der Beschreibung von Kompetenzen gerät die Didaktik in das Zentrum der Lehramtsstudiengänge in den Fächern. Die Kulturwissenschaften mit der Germanistik sind in diesem Rahmen einer wissenschaftlichen Ausbildung für den Beruf herausgefordert, über die vielfältigen Leistungen von Sprache und Wirkungen von Literatur in den verschiedenen Medien zu forschen und zu lehren. Einige Aspekte gemeinsamer Aufgaben einer Bildung durch Wissenschaften seien genannt:

- In der universitären Lehre gerät das erforschte und systematisch geordnete Wissen des Fachs immer auch unter der Frage ihrer angemessenen Vermittlung für Studierende und eine interessierte Öffentlichkeit in den Blick. 
- Die stets neue Diskussion über den Kanon des Wissens und der Literatur bewegt auch die Fachwissenschaften bei dem Versuch, Hierarchien des Wissens herzustellen. Um die Kompatibilität der Studiengänge zu sichern, behalten Inhalte der Lehrerbildung Einfluss auf die Master-Ausbildung.

- Bildungsromane machen einen bedeutenden Strang der Dichtung aus: vom Wilhelm Meister Goethes über den Grünen Heinrich Kellers, die Blechtrommel von Grass bis zum Spieltrieb von Juli Zeh oder die modernen Campus-Romane. Ebenso bedeutsam sind die ästhetischen Schriften: Von Schillers Briefe über die ästhetische Erziehung über Nietzsches Über die Zukunft der Bildungsanstalten, Brechts Theorie des Epischen und des Lehrtheaters bis hin zu Handkes Ich bin ein Bewohner des Elfenbeinturms. In Romanen, auf dem Theater und in theoretischen Abhandlungen der Dichter werden immer neue Antworten auf die Frage versucht, was die Literatur für das Leben leistet.

- Wissenschaft selbst wird zum Programm der Bildung. In der Schule und Hochschule lernen die jungen Menschen zu recherchieren, hypothetisch zu denken, rational zu begründen und das Arbeiten in methodischer Konsequenz und Sicherheit sowie das Werturteilen unter Vorbehalt. Wissenschaftliche Bildung wird zum wichtigsten „Bruchteil“ des seit Jahrtausenden andauernden „Intellektualisierungsprozesses“" (Max Weber, S. 18).

Mit guten Gründen lässt sich also auf der Ebene der Theoriebildung in den universitären Disziplinen, die den Auftrag zur Lehrerbildung angenommen haben, der Denkrahmen der Didaktik als einer Bildungswissenschaft beschreiben.

\subsection{Ebene 2: Theorie und Praxis: Handlungsforschung}

Die Stimme der Didaktik im Chor der Kulturwissenschaften ist zwar immer vernehmlicher zu hören, aber im Wettstreit mit den Fach- und Grundlagenwissenschaften sehe ich nicht ihre leitende Aufgabe. Die wesentliche Verpflichtung erwächst erst aus dem Nachdenken über gelingende Praxis in den Bildungseinrichtungen - im konkreten Fall der Forschung im Handlungsfeld des Deutschunterrichts allgemein- und berufsbildender Schulen. Es geht eben nicht mehr nur um die Beschreibung der Gesetzmäßigkeiten in den Bereichen von Sprache, Literatur und Kultur sowie den angemessenen Methoden der Beweisführung, sondern um den Nutzen der Kenntnisse und Methoden für gelingende Bildung der nächsten Generation in den entwickelten Institutionen. Auch dieses Aufgabenfeld kann als Ellipse mit zwei Brennpunkten veranschaulicht werden (Abbildung 2). 
Abb. 2: Angewandte Wissenschaften

Angewandte Wissenschaften

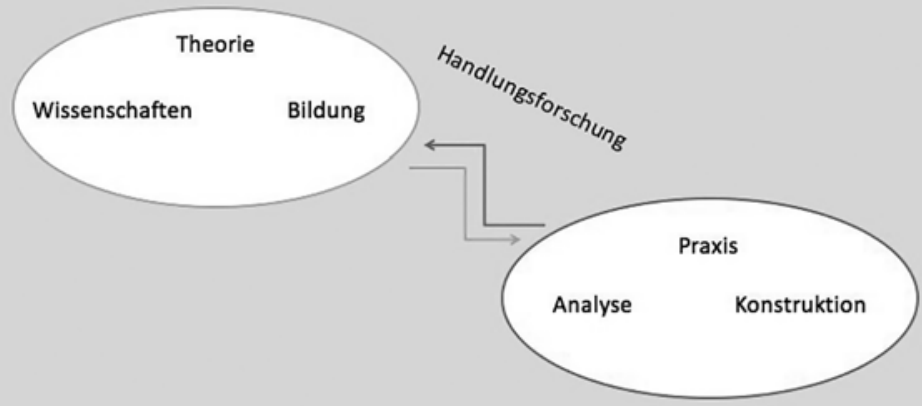

Wirksamer Unterricht lässt sich nur auf der Grundlage genauer Kenntnis der vorgefundenen Bedingungen und geltenden Praxis entwickeln. Der erste Brennpunkt der Forschungen im Schulfeld ist daher immer die Analyse des Ist-Standes in der Schule und der Lerngruppe. Eine der wichtigsten Bedeutungen der PISA-Studien liegt darin, dass sie alle Lehrenden und Forschenden kontinuierlich daran erinnert, wie entscheidend die Kenntnis über den Leistungsstand der SchülerInnen in einer Klasse ist, bevor der Unterricht beginnt. Im Zentrum der Analyse des Bedingungsfeldes für das Lehren und Lernen steht also die empirische Erforschung der konkreten sozialen, entwicklungspsychologischen Situation sowie des Leistungsvermögens der Lerngruppe. Darüber hinaus gilt die Analyse den Richtlinien, den Bildungsstandards sowie den Verordnungen der Kultusministerien, um die Diagonale zwischen allgemeinen staatlichen Vorgaben und den speziellen Ansprüchen der Lerngruppe für das bestmögliche Curriculum zu finden.

Ein Beispiel kann zeigen, wo die Analyse des Schulfeldes beginnt: Seit der Betreuung von Landschulpraktika im Oldenburger Land Ende der sechziger Jahre gehört zu meinen didaktischen Maximen die Expedition in die Orte und Wohngebiete der SchülerInnen, um das soziale und kulturelle Umfeld genau zu studieren. In dem Abschnitt „Architektur der Bildung“ in dem „Grundkurs Lehramt Deutsch“ habe ich nach einem Befund schwedischer Pädagogen den Raum als einen der drei Lehrer für die Erziehung und den Unterricht hervorgehoben: „Der erste sind die anderen Kinder. Der zweite Lehrer ist der Lehrer. Der dritte Lehrer ist der Raum" (Müller-Michaels 2009, S. 20-28). 
Im Kern sprechen wir aber, wenn von der Analyse der Bedingungen von Bildung und Unterricht die Rede ist, von empirischen Studien, meistens nach Maßgabe psychologischer, quantifizierender Methoden.

Der zweite Brennpunkt in der Ellipse der Praxis vor Ort ist die Konstruktion von curricularen Einheiten (Modulen, Bausteinen).

Konkret: Für mich ist der Entwurf von Planskizzen für einen anregenden und wirkungsvollen Deutschunterricht immer noch das Herzstück fachdidaktischer Arbeit, wie die Beiträge für die Zeitschrift „Deutschunterricht“ im Jahrgang 2015 belegen können: Ein Essay über „Geld und Poesie“ (H. 2) sowie Online-Module $\mathrm{zu}$ „Kriegsende 1945“ und zum Friedenspreisträger des Deutschen Buchhandels Navid Kermani. ${ }^{1}$ Grob gesehen unterscheiden wir zwei verschiedene Konstruktionsprinzipien: den system- und den problemorientierten Ansatz. Die systemorientierten Unterrichtseinheiten (Lehrgänge) knüpfen an die Wissensbestände der Fachwissenschaften an (Linguistik, Literaturwissenschaften, Publizistik mit Medienwissenschaften, Cultural Studies), die problemorientierten (Projekte) an die Lebenswelt der jungen Menschen und ihrer Mitwelt - entweder mit eher individuellen Problemen (Identität, Familie, Liebe und Tod) oder dominant universellen Fragen (Menschenrechte, Krieg und Frieden, Gerechtigkeit). Bedingung für die freie Planung von Unterricht ist allerdings, dass die Lehrpläne der Länder Spielräume für spezielle Unterrichtsentwürfe lassen, die an den konkreten Lernbedürfnissen und Interessen der Schülerinnen und Schüler ansetzen. Mit den immer präziser durchstrukturierten Bildungsstandards sind geraten wir hier an die freier Planung: Aber dem Ziel, die Gleichheit von Bildungschancen für alle jungen Menschen zu sichern, muss die Maxime übergeordnet sein, zu jeder Zeit Gerechtigkeit gegenüber jedermann walten zu lassen.

Neben Analyse und Konstruktion gibt es einen weiteren, zunehmend wichtigen Aspekt, der beide Brennpunkte im Rücklauf miteinander verbindet: die Evaluation von Modellen durch empirische Überprüfung ihrer Leistungsfähigkeit. Diese Verfahren können entweder kleinschrittig mit Blick auf konkrete Inhalte bzw. methodische Maßnahmen, großräumig in Langzeitstudien oder mit komplexen Fragestellungen zu Konzepten bzw. Problemlösungsstrategien angelegt sein. Grundsätzlich sind empirische Untersuchungen mit Hilfe quantitativer Methoden gleich wichtig wie qualitative Fallstudien, wenn sie aktuelle Befunde über laufenden Unterricht liefern. Der Vorteil der Case Studies ist allerdings, dass sie exemplarische Fälle liefern, Zusammenhänge der Lehr- und Lernprozesse sichtbar machen, kritisches Urteilen im konkreten Ablauf als Impuls für Handlungs-

1 Vgl. unter: www.d-unterricht.de/arbeitsblätter. 
anleitung in veränderter Praxis nutzen können. Was an Generalisierbarkeit der Befunde verloren geht, wird an Plausibilität gewonnen (Müller-Michaels 2013b). Die Forschungen im Praxisfeld lassen sich in drei Punkten zusammenfassen:

- Analyse des Bedingungsfeldes, z. B. Lernstandserhebungen, Richtlinien, Mitwelt, Architektur

- Konstruktion von Curricula, Systematischer vs. Projekt-Unterricht

- Experimente zur Prüfung von Modellen, Handlungsanleitungen

Mit den Andeutungen zu den Aufgaben von Schule und Unterricht auf der PraxisEbene ist deutlich geworden, wie eng für die Didaktik die Verbindung und die Spannungen zwischen den Denkrahmen von Theorie und Praxis ist. Die vielfältigen, nicht immer widerspruchsfreien Verknüpfungen begründen die Fachdidaktik als Handlungswissenschaft in dem Sinne, dass das von den Wissenschaften bereitgestellte Wissen im Schulfeld erprobt und mit Blick auf ihre Leistung für die Bildung kontinuierlich überprüft wird. Die Wahrheit über die Sachen ist etwas anderes als ihre Wirksamkeit für das zukünftige Leben der jungen Leute. Dem fachlichen Wissen wird eine entscheidende neue Perspektive hinzugefügt: seine Bedeutung für die Bildung der nächsten Generation und damit der Bewahrung der Kultur sowie ihrer immer neuen Aktualisierung in veränderten Zeiten. Der Weg von der Theorie zur Praxis ist dabei keine Einbahnstraße, vielmehr nehmen die Fragen der Praxis auch Einfluss auf die Theorieentwürfe (z. B. Kanon, Bildungssprache, methodische Bildung). In seinem Beitrag über das Verhältnis von Linguistik, Sprachdidaktik und Unterricht spricht Björn Rothstein von trilateralen Verbindungen und zeigt Denkbewegungen zwischen Theorie und Praxis im Anwendungsfeld Schule auf, fragt aber auch nach Leistungen der reinen Fachwissenschaften (2014).

Insofern Bildung und Unterricht das Anwendungsfeld der Germanistik bzw. Kulturwissenschaften ist, sprechen wir im Fall der Didaktik mit ihrer Doppelstruktur, von den Aufträgen zur Entwicklung und Begründung von Theorien und Modellen einerseits, andererseits zur Untersuchung geltender Praxis und Evaluierung innovativer Module, von Angewandter Wissenschaft. Schule ist ohne Frage das größte Anwendungsfeld der Geistes- und der meisten Naturwissenschaften und gibt den Disziplinen eine zusätzliche Verantwortung. Die Einrichtung von Zentren für Lehrerbildung bzw. Professional Schools of Education ist sinnfälliger Ausdruck der Doppelstruktur und des in den traditionellen Fakultäten gewachsenen Bewusstseins von der Bedeutung der Lehrerausbildung durch die Universitäten. In den Diskussionen der Schools of Education mit den Fakultäten lässt sich sinnfällig daran erinnern, dass Humboldt mit seinem Edikt „Über Prüfungen für das höhere Schulfach“ (11. April 1810) den Philosophischen Fakultäten die 
Aufgabe übertragen hatte, die Lehrerausbildung zu übernehmen. Mit dem Auftrag zur Ausbildung für einen Beruf hatte er die niedere Artes-Fakultät zur vierten Höheren Fakultät, neben Theologie, Jura, Medizin, erhoben. So verdanken die Geistes- und Naturwissenschaften ihren Status in der Universität auch der Lehrerausbildung. Berufsausbildung ist ein wesentlicher Teil akademischer Bildung.

\subsection{Ebene 3: Wissenschaftstheorie}

Möchten wir aber bekräftigen, dass es neben, über und vor der angewandten die reinen Wissenschaften gibt, die frei von allen Zwecken ausschließlich für weiterführende Erkenntnis forschen, dann landen wir bei der Wissenschaftstheorie als Denkrahmen (Abbildung 3).

\section{Abb. 3: Wissenschaftstheorie}

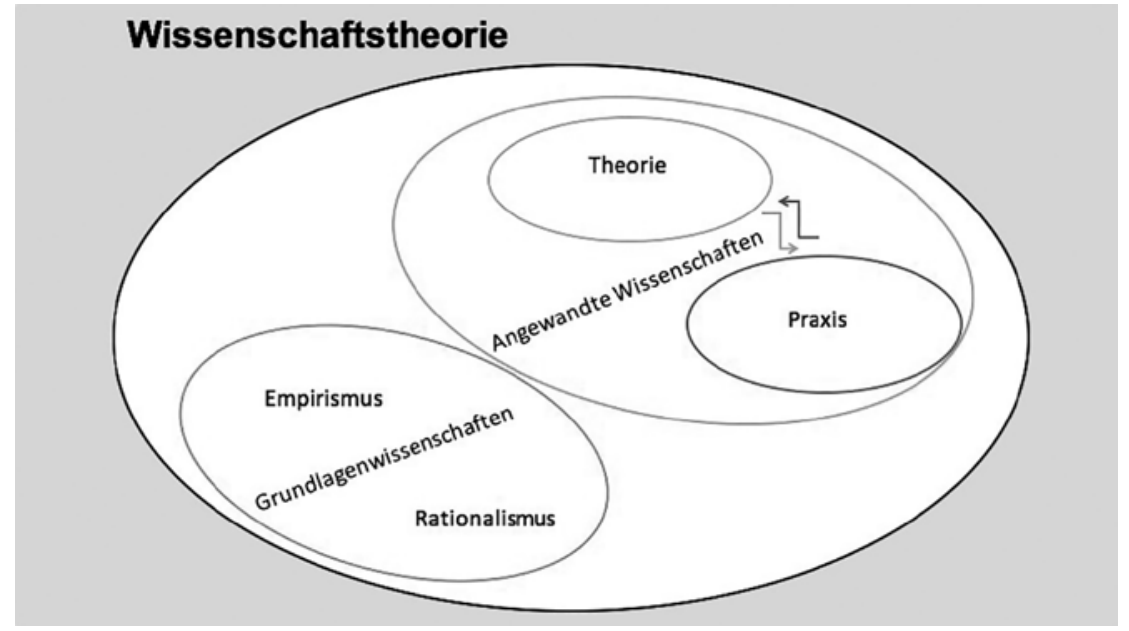

In diesem weiten Rahmen bilden Grundlagen- und Angewandte Wissenschaften wiederum eine Ellipse mit den zwei Brennpunkten. Bedenkt man die beschleunigte Entwicklung der Wissenschaften mit ihren zunehmend differenzierten Einsichten sowie ihre komplexe Reflexion durch die Philosophie, fällt es schwer, auch im Fall der zweckfreien Wissenschaften von nur einer Ellipse mit zwei Brennpunkten zu sprechen. Der Pluralität der Ansätze und Methoden entsprechend wäre es angemessener, weitere Denkrahmen für die Disziplinen in den Fakultäten anzunehmen und auf ein universalistisches Modell zu verzichten. In unserem Fall geht es zunächst allein um die Gegenüberstellung von Grundlagen- und 
Angewandter Wissenschaft, um deutlich zu machen, dass auch das Studium der reinen Wissenschaften in den Denkrahmen der Didaktik gehört. Trotz der Annahme weiterer Brennpunkte im weiten Feld der Wissenschaften soll in unserem Kontext an dem Bild der Ellipse festgehalten werden, um zu unterstreichen, dass Forschung sich mit ihren Aussagen immer in Zweifel stellt und Alternativen des Vorgehens bedenken muss, denn den einen Punkt, der zur Wahrheit führt, gibt es allenfalls auf Zeit. So setzen wir in die Ellipse der Grundlagenforschung die aus der Wissenschaftsgeschichte vertrauten Begriffe Empirismus und Rationalismus ein (Schurz 2006). Ohne an dieser Stelle auf die Unterschiede einzugehen, die vor allem in dem Realitätsgehalt von Aussagen liegen, die empirisch überprüft werden können oder nicht, bietet Karl Popper mit seiner Theorie der Falsifikation (2005) ein entscheidendes Kriterium an, mit dessen Hilfe sich Wissenschaft von Spekulation unterscheiden lässt.

Dem präzisen Denkrahmen der Grundlagenwissenschaften im Kontext von Lehrerausbildung Raum zu geben, liegt in der Notwendigkeit begründet, auch die Kompetenzen der Studierenden für die Teilhabe an zweckfreier Forschung zu fördern. Unbestritten ist, dass didaktische Diskussionen immer wieder theoretischen Anregungen aus den Grundlagenwissenschaften folgen, die zu einflussreichen Diskursen auch in Theorie und Praxis des Deutschunterrichts führen, wie z.B. die Kritischen Gesellschaftstheorien in den Siebzigern, die Medientheorien in den Achtzigern und die Kognitionspsychologie seit PISA 2000. Die fachdidaktischen Debatten werden immer wieder neu angeregt und formulierten neue Konzepte, die auf ihre Geltung geprüft wurden. Wichtigstes Kriterium der Prüfung ist der Kernauftrag der Didaktik, die ganzheitliche Bildung der jungen Menschen argumentativ und durch Lernmodelle zu befördern und sich nicht kritiklos einem modischen Paradigma zu unterwerfen. Sofern Didaktik Reflexionen ihres eigenen Denkens und Handelns vorantreibt, ohne unmittelbar an konkrete Verwertungszusammenhänge zu denken, operiert auch sie als reine Wissenschaft.

Noch bedeutsamer für den zukünftigen Fachunterricht werden neue Einsichten aus den Sprach-, Literatur- und Medienwissenschaften mit ihren innovativen Theorieentwürfen, veränderter Systematik der Gegenstände, neuen Editionen von Werken mit profunden Kommentaren, weiterführenden Interpretationen und bedenkenswerten Wertungen. Folgt man dem Konzept der formalen Bildung, dann lässt sich über die Leistung fachwissenschaftlicher Studienanteile sagen: Wer sich während seines/ihres Studiums in den Fach- und Bezugswissenschaften auf komplexe Probleme eingelassen und in Examensarbeiten exemplarisch gelöst hat, wird mit Problembewusstsein wirkungsvoll auch neue Herausforderungen 
bestehen. Um es pointiert zu formulieren: Wer seine Master-Arbeit zu einer Fragestellung aus Robert Musils Mann ohne Eigenschaften geschrieben hat, wird sich auch, aus einem weiten Denkhorizont, so kompetent in das neue Problemfeld „Inklusion“ einarbeiten, dass sie/er sehr schnell zu den Spezialisten aufschließt. Aus fachlicher Qualifikation wächst Autorität und Kompetenz. In den Studien von Linguistik und Literaturwissenschaft lernen die angehenden LehrerInnen ihr Grundlagen- und Methodenwissen anzuwenden, interdisziplinäre Probleme zu erkennen, für ihre konkreten Aufgaben nutzbar zu machen, überprüfbare Argumentationen zu entwickeln und reflektiert, der konkreten Verwendung bewusst, darzustellen. Diese wissenschaftlichen Kompetenzen qualifizieren auch für die Forschung im Anwendungsfeld Schule. Umgekehrt gilt allerdings auch, dass die Befähigung zur angewandten Forschung dem Studium der reinen Wissenschaften zusätzliche Bedeutung verleiht.

\section{Diskurse der Didaktik}

Entsprechend ihrem Ort im Gefüge der Wissenschaften und den leitenden Erkenntnisinteressen haben sich in der Didaktik unterschiedliche Diskurse entwickelt, die streng nebeneinander stehen oder, wie bei dieser Tagung, miteinander ins Gespräch gebracht werden. Mit Jürgen Habermas habe ich Diskurs als Form der Kommunikation verstanden, in der sprachliches Handeln zugleich auf der Metaebene problematisiert wird: „In Diskursen suchen wir ein problematisiertes Einverständnis, das im kommunikativen Handeln bestanden hat, durch Begründung wiederherzustellen: In diesem Sinne spreche ich fortan von (diskursiver) Verständigung“" (Habermas 1971, S. 115). Im erweiterten Sinne definiert Manfred Titzmann Diskurs als „System des Denkens und Argumentierens, das erstens durch einen Redegegenstand, zweitens durch Regularitäten, drittens durch interdisziplinäre Relationen zu anderen Diskursen charakterisiert ist" (Titzmann 1991, S. 406). Auf der Grundlage einer solchen Bestimmung durch Gegenstand, Redeform und Bezug zu anderen Wissenschaften habe ich, Herbert Schnädelbach (1977) folgend, zunächst drei Diskurse unterschieden: den explikativen, den deskriptiven und normativen (Müller-Michaels 2016, S. 272-293).

Im explikativen Diskurs (theoretisches Traktat) werden grundlegende Begriffe, Konzepte sowie Methoden erläutert und für den Gebrauch begründet. Exemplarisch sei an Manfred Fuhrmanns Abhandlung über Der europäische Bildungskanon und die deutsche Bildungsidee (2002) erinnert, in dem er die Begriffe „Kultur“ und „Bildung“ dergestalt einander gegenüberstellt, dass Kultur den gesamteuropäischen Kanon des Wissens, Bildung hingegen den spezifisch deutschen Zugang über den Prozess der Aneignung des Kanons durch die In- 
dividuen meint; dieser Idee folgt auch die von mir vorgeschlagene Definition (Müller-Michaels 2009, S. 42): Bildung ist sowohl als Prozess wie als Resultat zu verstehen. Die Begriffe „Bildung“ und „Erziehung“ in ihrer langen Geschichte seit den jüdischen Schriften im Alten Testament und der antiken griechischen Philosophie erläutert Käte Meyer-Drawe in ihrem Aufsatz Zum metaphorischen Gehalt von ,Bildung' und,Erziehung'. Sie kommt zu dem Befund: „In der Moderne betont Bildung den Menschen ausschließlich als ,Werk seiner selbst‘. Selbstbildung meint Subjekt und Substanz." (Meyer-Drawe 1999, S. 171) Gleichwohl wird deutlich, dass das semantische Potenzial der Begriffe von Ebenbildlichkeit versus Bildnisverbot sowie Zucht gegen Befreiung im Kontext ihrer Verwendungen über die Jahrhunderte hinweg immer noch mitschwingt.

Sind die beiden Traktate zu Geschichte und Leistungen der Bildungsidee auch unabhängig von Anwendungen zu praktischem Gebrauch denkbar, so rückt die Abhandlung von Franz Erich Weinert Vergleichende Leistungsmessung in Schulen - eine unbestrittene Selbstverständlichkeit (2001) in den Brennpunkt der Bildungswissenschaften zur Rechtfertigung Quantitativer Untersuchungen im Schulfeld. Gegenüber den hochbewerteten reformpädagogischen Ideen verteidigt er konsequente empirische Forschung. Den Leistungsprüfungen kommen demnach sowohl für die Ermittlung von Wissen und Können einer Lerngruppe als auch für eine den Schülern angemessene differenzierte Planung von Bildungsmaßnahmen zu. In dem genannten Beitrag wird auch zum ersten Mal der Kompetenzbegriff als Messgröße für die Leistungsprofile eingeführt, in dem sich aber, anders als bei den meisten nachfolgenden Studien, der Gesamtauftrag der Bildung und Erziehung nicht erschöpft.

Im deskriptiven Diskurs (empirische Studien) geht es vor allem um quantifizierende und qualitative Untersuchungen im Schulfeld - sowohl um die Erfassung des Leistungsstandes einer Lerngruppe oder Alterskohorte weltweit als auch um die Prüfung eines Unterrichtsmodells in einem schulischen Experiment. Als Beispiel scheint mir an dieser Stelle Heiner Willenbergs Untersuchung zu „Lesen“ aus der DESI-Studie besonders geeignet, weil er das Modell der Kompetenzstufen mit Blick auf den Gegenstand schlüssig revidiert hat:

- Identifizieren einfacher Lexik

- Lokale Lektüre

- Verknüpfende Lektüre

- Mentale Modelle bilden (Willenberg 2007, S. 109 f.)

Hingewiesen sei an dieser Stelle auf den Beitrag Michael Krelle in diesem Band, der als Beispiel eines neuen deskriptiven Diskurses in diesem Sinne aufgefasst werden kann. 
Besonders wichtig ist mir, dass wir über allen quantitativen Leistungsmessungen die qualitative Unterrichtsforschung mit ihren Fallstudien aus Schulen, zu Problemen (Einwanderer, Geschlechter, Mehrsprachigkeit etc.), zur Lehrerausbildung nicht abwerten und für die ich in meinem Beitrag in der Festschrift für Gerhard Rupp unter dem Titel Gleichheit oder Gerechtigkeit? - Ziele der Bildung und empirische Forschung (2013b) nachdrücklich argumentiert habe.

Insofern es in der Didaktik meistenteils darum geht, innovative Inhalte, Prinzipien (wie z. B. Gleichbehandlung, Würde, Zumutung) und/oder Methoden für den Unterricht vorzuschlagen und zu erproben, gewinnen die normativen Diskurse (didaktische Programmatik) besonderes Gewicht. In diesem Redetyp geht es nicht mehr um die Beschreibung ermittelter Tatsachen, sondern um die Begründung von Entscheidungen, welche Inhalte und Verfahren heute für die gesetzten Ziele von Bildung und Erziehung notwendig erscheinen. In den normativen Diskursen werden Geltungsansprüche verteidigt und durchzusetzen versucht. Die Redeweise in den programmatischen Schriften ist häufig gekennzeichnet durch setzende Diktion, Anschluss an empirische Befunde oder individuelle Erfahrungen, Bekräftigung von administrativen Vorgaben, Anregungen aus den Bezugswissenschaften und Appelle zur Umsetzung von Planungsskizzen in die Praxis. Ein bekanntes Beispiel für diesen Diskurstyp ist die Einführung Mediendidaktik Deutsch von Volker Frederking et al. (2008). Das Werk lässt erkennen, wie entschieden die Aufgaben des Deutschunterrichts über den Rahmen traditioneller Germanistik hinausgreifen müssen. Bei aller denkbaren Kritik erfüllt das Buch den Zweck, Aufmerksamkeit zu wecken, den neuesten Stand der Diskussion über ein aktuelles Thema zu reflektieren, Konzepte vorzustellen und zahlreiche Beispiele der unterschiedlichen Medienformate zu erläutern. Wenn allerdings behauptet wird, dass die Medien, neben Sprache und Literatur, die dritte Säule der Deutschdidaktik darstellen, so ist das, vorsichtig gesagt, als übertriebene Form der Aufmerksamkeitssteuerung zu werten, denn die dritte Säule ist immer noch die Rhetorik des Redens und Schreibens. Medien sind allenfalls Mittel für den methodischen Umgang mit den Sachen, die durch die Neuen Medien nur am Rande bereichert werden.

Ein zweites Beispiel für den normativen Diskurs wird an anderer Stelle (vgl. Müller-Michaels 2016) ausführlicher erläutert: Gerd Bräuers innovatives Werk Schreibend lernen. Grundlagen einer theoretischen und praktischen Schreibpädagogik (1998). Wir dürfen es insofern als exemplarisch ansehen, als es seinen Gegenstand induktiv, fragend-entwickelnd, behutsam konkretisierend und theoretisch zusammenfassend in reflektierten Schritten, vom ersten Schreibversuch mit GrundschülerInnen bis zum Portfolio am Ende der Schulzeit entfaltet. Solche 
Art der allmählichen Verfertigung eines Modells durch ein im Anspruch an die Lernenden wachsendes Handeln bleibt eine Herausforderung für eine wirkungsvolle Didaktik.

\section{Zusammenfassung}

Die Beispieldiskurse aus der Deutschdidaktik können nur idealtypisch verstanden werden, denn die meisten Abhandlungen werden Mischformen darstellen: Der explikative Diskurs wird mit Fällen aus der Praxis angereichert, der deskriptive Diskurs mündet in konkrete Empfehlungen für innovative Unterrichtsmodelle, der normative Diskurs benutzt Argumente aus theoretischen Traktaten. Dennoch sollte deutlich werden, dass wir in der Didaktik unterschiedliche Redeformen verwenden und uns daher häufig genug missverstehen. Sich dieser Diskursformate bewusst zu sein und neben der Diskussion über die Inhalte und die Ergebnisse der Forschungen auch über die Formen ihrer Darstellung zu reden, ist ein Auftrag gerade für Vertreter der Sprach- und Kulturwissenschaften.

Unbestritten ist auch, dass es weitere Redeformate gibt; an anderer Stelle werde ich die appellativen (Essays, Leitartikel, Kommentare) und die administrativen Diskurse (Lehrpläne, Bildungsstandards) zusätzlich erläutern (vgl. MüllerMichaels 2016). Im Kontext dieses Beitrags kam es aber zunächst darauf an, die Pluralität der Diskurse über Bildung und Unterricht herauszustellen. Diese Diskurse wachsen aus den verschiedenen Kontexten unserer Forschungen, die mit den drei Ellipsen beschrieben wurden. Die Brennpunkte der Forschungsaufträge bilden die Zentren in dem präzisierten Denkrahmen für die Vorhaben: die Wirklichkeit von Schule und Unterricht zu beschreiben, die notwendigen Modelle für das Lehren und Lernen zu entwickeln, den Anschluss an die neueren Forschungen und Paradigmen der Bezugswissenschaften zu behalten und die Befunde auf ihre Leistung für die Bildung der nächsten Generation zu prüfen sowie Empfehlungen für die Praxis zu artikulieren, schließlich immer auch das eigene Denken und Handeln zu reflektieren und offensiv in das Gespräch der Wissenschaften einer Universität zu tragen. Diese Selbstreflexion der Reichweiten didaktischer Forschungen sowie der gelingenden Lehre begründen den weiten Denkrahmen.

Hölderlin hat im Vorwort zum Hyperion in der Thalia-Version (1794, Werke Bd. 3) die Ellipse als Urform menschlichen Lebens und Strebens bezeichnet, die auf Bewegung (in „exzentrischer Bahn") zur Vollendung der Bildung dringt (Hagel 2003, S. 269). Was hier als Weg zu immer höherer Stufe der Entwicklung verstanden ist, kann auch für die Didaktik als Wissenschaft gelten: Ständige Bewegung und fortschreitende Erkenntnis zwischen den Polen auf den horizontalen Ebenen und vertiefender Reflexionen auf der vertikalen Achse vom Praxisfeld 
über die Fundierung durch die Wissenschaften bis hin zur Wissenschaftstheorie als Legitimation der Fragestellungen. Diese Bewegungen auf den verschiedenen Ebenen markieren den weiten Denkrahmen der Didaktik. Die Pluralität der Diskurse begründet unser nicht nachlassendes Interesse an der Disziplin: das ständige Auf und $\mathrm{Ab}$ der Problemstellungen zwischen Praxis und Theorie, die gedankliche Bewegung zwischen den Brennpunkten auf den verschiedenen Ebenen des Fragens und Forschens, die Verantwortung für die Bildung der Heranwachsenden sowie die Wahl der angemessenen Diskursform. Die variationsreiche Arbeit stärkt die Gewissheit, dass die erarbeiteten Lösungen nur auf Zeit gelten und neue Impulse für die Forschung auslösen werden.

\section{Literatur}

Bräuer, Gerd (1998): Schreibend lernen. Grundlagen einer theoretischen und praktischen Schreibpädagogik. Innsbruck/Wien: Studien-Verlag.

Frederking, Volker/Maiwald, Klaus/Krommer, Axel (2008): Mediendidaktik Deutsch. Eine Einführung. Berlin: Schmidt.

Fuhrmann, Manfred (2002): Bildung. Europas kulturelle Identität. Stuttgart: Philipp Reclam.

Habermas, Jürgen (1971): „Vorbereitende Bemerkungen zu einer Theorie der Kommunikativen Kompetenz." In: ders./Luhmann, Niklas (Hrsg.): Theorie der Gesellschaft oder Sozialtechnologie - Was leistet die Systemforschung? Frankfurt: Suhrkamp.

Hagel, Ulrike (2003): Elliptische Zeiträume des Erzählens. Würzburg: Königshausen \& Neumann.

Hölderlin, Friedrich (1957/1961): „Hyperion“ und „Über die Verfahrensweise des poetischen Geistes“. In: Beissner, Friedrich (Hrsg.): Hölderlin. Gesammelte Werke. Stuttgart: W. Kohlhammer Bd. 3, S. 163, Bd. 4, S. 241-265.

Humboldt, Wilhelm von (1996): „Über Prüfungen für das Höhere Schulfach“. In: Flitner, Andreas/Giel, Klaus (Hrsg.) : Wilhelm von Humboldt, Werke in Fünf Bänden Bd. IV. [5. Aufl.] Darmstadt: Wissenschaftliche Buchgesellschaft, S. 241-244.

Meyer-Drawe, Käte (1999): „Zum metaphorischen Gehalt von ,Bildung und ,Erziehung “'. In: Zeitschrift für Pädagogik. Jg. 45. H.2, S. 161-175.

Müller-Michaels, Harro (2009): Grundkurs Lehramt Deutsch. Stuttgart: Klett Lerntraining.

Müller-Michaels, Harro (2013a): „Wettstreit der Diskurse - Von den Niederlagen der Didaktik“. In: Dawidowski, Christian (Hrsg.): Bildung durch Dichtung - 
Literarische Bildung. Bildungsdiskurse literaturvermittelnder Institutionen um 1900 und um 2000. Frankfurt a. M. u. a.: Peter Lang.

Müller-Michaels, Harro (2013b): „Gleichheit oder Gerechtigkeit? Ziele der Bildung und empirische Forschung“. In: Frickel, Daniela A./Boelmann, Jan (Hrsg.): Literatur-Lesen-Lernen. Festschrift für Gerhard Rupp. Frankfurt a. M. u. a.: Peter Lang, S. 259-272.

Müller-Michaels, Harro (2016): „Diskurse über Bildung 2000 - am Beispiel der Didaktik“. In: Kilian, Jörg/Brouër, Birgit/Lüttenberg, Dina (Hrsg.): Handbuch Sprache in der Bildung. Berlin u. a.: De Gruyter, S. 272-293.

Popper, Karl (2005): Logik der Forschung. Hrsg.v. Keuth, Herbert [11. Aufl.], Tübingen: Mohr Siebeck.

Rothstein, Björn (2014): „Auf dem Weg zu einer Dreiecksbeziehung? Das angestrengte Verhältnis von Linguistik, Sprachdidaktik und Unterricht“. In: Rödel, Michael (Hrsg): Was kann die Sprachwissenschaft leisten? Hohengehren: Schneider.

Schnädelbach, Herbert (1977): Reflexion und Diskurs. Fragen einer Logik der Philosophie. Frankfurt a. M.: Suhrkamp.

Schurz, Gerhard (2006): Einführung in die Wissenschaftstheorie. Darmstadt: Wissenschaftliche Buchgesellschaft.

Tenorth, Heinz-Elmar (1994): „ALLE ALLES ZU LEHREN“. Möglichkeiten und Perspektiven Allgemeiner Bildung. Darmstadt: Wissenschaftliche Buchgesellschaft.

Titzmann, Michael (1991): Modelle des literarischen Strukturwandels. Tübingen: Niemeyer.

Weber, Max (1995): Wissenschaft als Beruf. Stuttgart: Philipp Reclam (UB 9388). Weinert, Franz E. (2001): Leistungsmessungen in Schule. Weinheim und Basel: Beltz.

Willenberg, Heiner (2007): „Lesen“. In: Beck, Bärbel/Klieme, Eckhard (Hrsg.): Sprachliche Kompetenzen. Konzepte und Messung. DESI-Studie (Deutsch Englisch Schülerleistungen International). Weinheim und Basel: Beltz, S. $107-117$. 
Michael Krelle

\title{
Deutschdidaktik als (zu) gestaltende Wissenschaft? \\ German didactics - a science that shapes or needs shaping?
}

\begin{abstract}
The paper outlines the scientific-theoretical form of German didactics. Citing works by Jakob Ossner, the author advocates perceiving the discipline as a practical science to which a specific thought style is tied. Reference variables for specifying such a common thought style in more detail are suggested.
\end{abstract}

\section{Perspektiven}

Die Diskussion um das Selbstverständnis der Deutschdidaktik ist nicht neu; sie wird seit geraumer Zeit mit unterschiedlicher Intensität geführt (vgl. u. a. Ivo 1975, 1977; Ossner 1993; Hebel 1993; Kepser 2013; Frederking 2014). Strittig ist insbesondere der wissenschaftstheoretische Ort der Disziplin: Ist die Deutschdidaktik eine Wissenschaft eigener Art, eine bestimmte Form angewandter Germanistik oder eine mehrere Disziplinen zusammenspannende „Bindestrichwissenschaft"? (Bremerich-Vos 1993, S. 1). Bei den jeweiligen Verortungen wird mitunter von einer „Zwischen-den-Stühlen-Disziplin“ gesprochen (u. a. Winkler 2012, S. 9). Das ist sicherlich keine schmeichelhafte Metapher, wie Kepser festhält (2013, S. 52): „Zwischen den Stühlen sitzt man bekanntlich auf dem Boden“, was man allerdings auch als „Erdung“ ansehen kann (ebd.).

Das Problem der Verortung ist in Teilen hausgemacht: KollegInnen anderer Disziplinen nehmen DeutschdidaktikerInnen in der Regel nicht als ExotInnen wahr. In der Außenperspektive gehören DeutschdidaktikerInnen vielmehr zu der Gruppe von Menschen, die im Kontext von Hochschulen und/oder im Rahmen von Fortbildungen für Deutschunterricht und seine Didaktik zuständig ist. Vor dem Hintergrund von Sprachbildung als Aufgabe des Unterrichts in allen Fächern (Schneider u.a. 2012, S. 23) sind DeutschdidaktikerInnen darüber hinaus weit über die Grenzen des Faches Deutsch hinaus gefragt. Das betrifft dann neben der schulischen auch Aspekte der vorschulischen und außerschulischen Betätigung und bezieht sich auf unterschiedliche „Rahmungen von Unterricht“ (vgl. Bräuer 2011). In der Alltagspraxis ist man als DeutschdidaktikerIn zudem häufig zusam- 
men mit KollegInnen anderer Disziplinen auf verschiedenen Tätigkeitsfeldern aktiv, vor allem im Rahmen der Lehramtsausbildung an Hochschulen sowie im Kontext von Fortbildungen für Lehrkräfte und ReferendarInnen. DeutschdidaktikerInnen beteiligen sich auch an fachübergreifenden und bildungspolitischen Diskursen, an der Gestaltung von Curricula, Standards etc. Sie führen empirische Klein- und Großprojekte mit ganz eigenen genuin deutschdidaktischen oder aber auch fachübergreifenden Fragen durch. Zudem gehen Deutschdidaktikerinnen nicht nur mit Normen um, die an sie herangetragen werden. Die Deutschdidaktik ist im Wesentlichen auch selbst normsetzende Instanz (vgl. den Beitrag von Dorothee Wieser in diesem Band). Im Hinblick auf das Tagungsthema zum Symposium Deutschdidaktik 2014 in Basel heißt es dazu:

Die Gesellschaft erwartet vom Deutschunterricht eine sprachliche und literarische Bildung, die Deutschdidaktik selbst legitimiert und modifiziert sprachliche und literarische Traditionen der Gesellschaft. Sie orientiert sich dabei einerseits an den gesellschaftlichen Normen bzw. Erwartungen, andererseits an der Unterrichts- und Lernwirklichkeit. (SDD 2014)

Obwohl also DeutschdidaktikerInnen in der Außenperspektive einen festen Platz im Wissenschaftsbetrieb einnehmen, wird die Diskussion um das Selbstverständnis innerhalb der Disziplin weiterhin geführt: „Man kann fast sagen, dass sie sich seit ihrer Entstehung auf der Identitätssuche befindet", so Winker (2012, S. 9).

Vor diesem Hintergrund wird im Folgenden metaphorisch zwischen einem ,inneren' und einem ,äußeren' Ort unterschieden. Der ,äußere Ort' ergibt sich aus den an die Deutschdidaktik gestellten Ansprüchen bzw. aus den gesellschaftlich zugewiesenen Aufgaben. Dieser Ort ist wenig umstritten. Der ,innere Ort" (') um in dieser Metapher zu bleiben - ist hingegen ein gemeinsamer Rahmen, der die Deutschdidaktik als Kollektiv identifizierbar macht (vgl. Ossner 2001, S. 23). Dieser ,Ortskern' ist deutlich umstrittener.

\section{Ortsbesichtigungen}

Ein zentraler Ort, an dem DeutschdidaktikerInnen organisiert sind, ist das Symposion Deutschdidaktik e. V. (SDD). Auf der Internetseite des Vereins finden sich die folgenden Leitlinien ${ }^{1}$ :

- Wissenschaft: Im SDD sind Wissenschaftlerinnen und Wissenschaftler organisiert, die Konzepte über sprachliche und literarische Lehr- und Lernprozesse weiterentwickeln.

1 Vgl.SDD: http://www.symposion-deutschdidaktik.de/home. Abgerufen am 01.04.2016. 
- Lernende: Wir arbeiten dafür, dass Lernende jeder Herkunft und Begabung im Fach Deutsch möglichst gute Lernangebote vorfinden.

- Lehrkräfte: Wir bilden Lehrkräfte aus und weiter, die den Deutschunterricht als Ort der Begegnung mit Sprache, Literatur und Medien verstehen und nutzen können.

- Bildungspolitik: Wir vertreten die Interessen und Anliegen unserer Hochschuldisziplin auch im bildungspolitischen Diskurs.

- Deutsch: Uns interessiert die Vergangenheit, die Gegenwart und vor allem die Zukunft des Faches Deutsch in allen Schularten und Schulstufen.

- Mitglieder: Wir haben Mitglieder überall, wo Deutsch als Erst- und Zweitsprache gelehrt wird - in Deutschland, Österreich, der Schweiz und Südtirol.

Hier sind - stark verkürzt - die wesentliche Ziele deutschdidaktischer Arbeit zusammengefasst, die sich im Hinblick auf den oben genannten, äußeren Ort' beziehen; sie hängen auch mit den unterschiedlichen Aktanten im „Handlungsfeld Deutschunterricht" zusammen (Ivo 1975): Im Hinblick auf Lernende und Lehrende geht es um die Erforschung und Gestaltung möglichst wirksamer, motivational förderlicher bzw. ,guter' Lernangebote und ihren Nutzen sowie um eine umfassende Lehrerbildung und -fortbildung mit Blick auf Sprache, Literatur und Medien, und zwar überall dort, wo Deutsch als Erst- und Zweitsprache gelehrt und gelernt wird. Im Hinblick auf WissenschaftlerInnen geht es um die Konstruktion, Weiterentwicklung und Überprüfung von Konzepten sowie Modellen sprachlicher und literarischer Lehr- und Lernprozesse. Dabei sind dann insbesondere auch eine historische sowie eine bildungspolitische Perspektive leitend. Oder allgemeiner gesagt: Die Deutschdidaktik ist eine Wissenschaft, die Antworten auf die Frage gibt: Was soll ich im Deutschunterricht tun und wie? Dafür stellt sie sowohl Ziele und Zielkategorien als auch für das Erreichen notwendige Handlungen und Handlungsalternativen zur Verfügung (Ossner 2001, S. 27).

Bereits an diesen Verallgemeinerungen lässt sich ablesen, dass an diesem ,äußeren Ort' KollegInnen mit sehr unterschiedlichen Perspektiven versammelt sind. Gerade diese Pluralität ist der Motor für eine produktive Zusammenarbeit und Weiterentwicklung der Disziplin. In der Einleitung des Tumulte-Bandes von Rosebrock/Fix (2001) heißt es dementsprechend:

Freilich ist die dissonante Vielstimmigkeit, mit der vor verschiedenen Horizonten und aus unterschiedlichen Perspektiven die akademische Identität der Fachdidaktik Deutsch proklamiert oder konstruiert wird, nicht nur Grund zur Klage: Der Tumult ist eben auch historisch oder systematisch der Schauplatz des Paradigmenwechsels, also der Ausgangspunkt der Neukonstitution eines disziplinären Selbstverständnisses. (Rosebrock 2001, S. 5) 
Insofern spricht sehr viel dafür, die Pluralität der Deutschdidaktik im äußeren Ort als Chance für die Gestaltung des inneren Ortes zu begreifen: Vorschläge, die Deutschdidaktik „,im Kern“ ausschließlich als Teildisziplin oder als Appendix der Germanistik zu verorten (vgl. z. B. Hebel 1993; Müller-Michaels 2001), greifen vor diesem Hintergrund zu kurz; die Vorstellung einer "germanistischen Mutterwissenschaft" ist nicht haltbar, wie etwa Abraham/Kepser (2005, S. 43) zusammenfassen. Kepser (2013) versucht deshalb auch, die unterschiedlichen Perspektiven unter dem Dach der „Kulturwissenschaft“ zu vereinen. Allerdings ist auch dieser Bestimmungsversuch unbefriedigend (Frederking 2014, S. 112): Der Begriff der Kulturwissenschaft ist nämlich „[...] ebenso unscharf wie exklusiv, erscheint er doch als Oberbegriff für alle Fachdidaktiken nicht oder doch nur sehr eingeschränkt tauglich. Insofern erfasst er nicht den wissenschaftlichen Status aller Fachdidaktiken“ (ebd.). Frederking (2014) schlägt deshalb unter Bezugnahme auf Mittelstraß (1992) vor, die Deutschdidaktik im Kern als transdisziplinäre Wissenschaft zu verstehen. Das heißt: In ihr werden zwar Erkenntnisse und Methoden aus dem Forschungsfeld der Fachwissenschaft bzw. der Germanistik verarbeitet, aber auch solche aus Bezugswissenschaften wie Pädagogik, Psychologie, Philosophie, Soziologie, Geschichte und anderen Fachdidaktiken. Mit Blick auf deutschdidaktische Forschung heißt das:

Auf dieser Grundlage zielt deutschdidaktische Forschung auf die konzeptionelle Entwicklung, theoretische Fundierung und empirische Erforschung fachspezifischer Lehr-LernProzesse und ihrer inhaltlichen und methodischen Grundlagen innerhalb und außerhalb der Schule. (Frederking 2014, S. 111)

In einer solchen Konzeption ist die Deutschdidaktik nicht als Disziplin „Zwischen den Stühlen“ profiliert. Sie ist auch kein Teil der Germanistik, jedoch ist die Germanistik auch nicht eine von vielen Bezugswissenschaften der Deutschdidaktik. Vielmehr werden im Wesentlichen die Erkenntnisse und Methoden aus dem Forschungsfeld der Fachwissenschaft (hier der Germanistik) berücksichtigt, zweitens aber auch solche aus Bezugswissenschaften wie der Pädagogik, Psychologie, Philosophie, Soziologie, Geschichte, anderen Fachdidaktiken etc. in Betracht gezogen (ebd., S. 111). Sie steht damit gewissermaßen in einem „Netz von Bezugswissenschaften, deren Gegenstände und Methoden für die Erforschung der Bedingungsfelder von Schule und Erziehung sowie der Entscheidungsfelder von Unterricht und Bildung unverzichtbar sind“, wie es Müller-Michaels (2012, S. 15) nennt. Jedoch: Die Germanistik ist hier die fachwissenschaftliche Bezugsgröße der Deutschdidaktik, nicht irgendeine (beliebige) Bezugswissenschaft (vgl. Abbildung 1). 
Abb. 1: Deutschdidaktik transdisziplinär (in Anlehnung an Frederking 2014, S. 111)

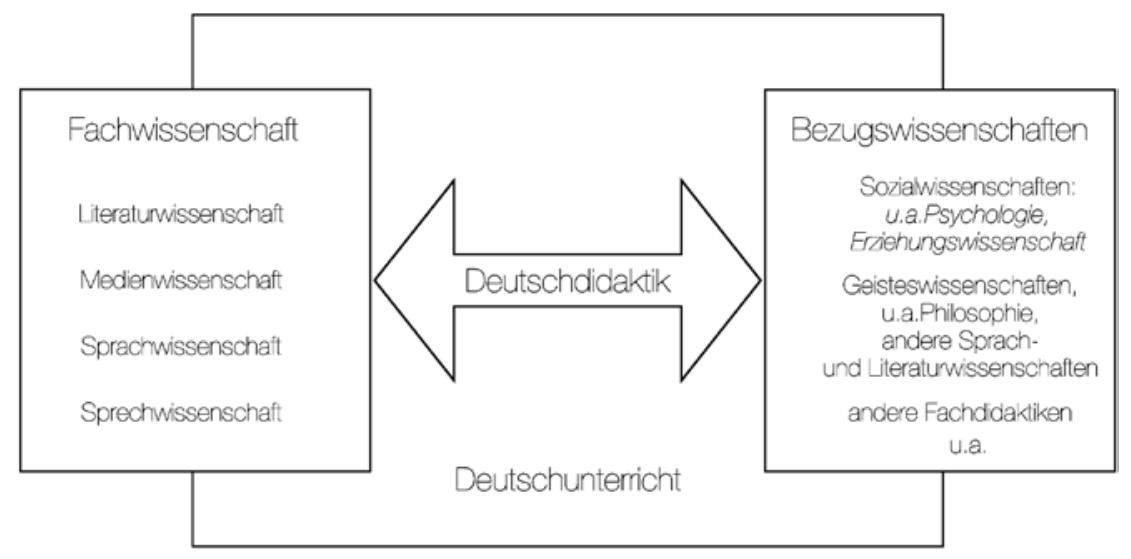

Dass der Deutschunterricht hier anders als bei Frederking (2014) als Rahmung auftritt, ist bewusst gewählt: Auch in den genannten Bezugswissenschaften (z. B. in der pädagogischen Psychologie) und in der Fachwissenschaft werden mitunter Aspekte des Deutschunterrichts untersucht. Dieses geschieht aber nicht in fachdidaktischer Zielperspektive. Zudem gilt: Während sich andere Disziplinen (z. B. die Psychologie) als empirische Wissenschaften etablieren können, „ist dies bei der Fachdidaktik in derselben Weise nicht der Fall. Zwar ist Empirie ein notwendiger Bestandteil der Fachdidaktik, aber sie allein reicht nicht zur Bestimmung des Feldes aus." (Ossner 2001, S. 18)

Um nicht falsch verstanden zu werden: Häufig sind DeutschdidaktikerInnen natürlich auch gemeinsam mit PsychologInnen an Studien beteiligt. Im Rahmen des Bildungsmonitorings hat etwa die Kultusministerkonferenz (KMK) regelmäßige „Ländervergleiche“ beschlossen. Ziel dieser Studien ist es festzustellen, inwieweit SchülerInnen in Deutschland die nationalen Bildungsstandards erreichen und in welchen Bereichen Steuerungsbedarf besteht (vgl. Pöhlmann u. a. 2009). Hier arbeiten deutschdidaktische KollegInnen mit PsychologInnen zusammen, u. a. um passgenaue Aufgaben zu entwerfen. Diese Arbeit ist allerdings zunächst keine genuin deutschdidaktische. Hier wird vielmehr deutschdidaktische Expertise in bildungspolitische Prozesse eingebracht, die dann vermittelt wiederum auf den Deutschunterricht und seine Didaktik wirken können. Auch solche Tätigkeiten zählen zum, äußeren Ort'.

Wenn es um eine genuin fachdidaktische Zielperspektive bzw. den ,inneren Ort' geht, ist hingegen ein - mehr oder weniger - gemeinsamer Denkrahmen bedeutsam. Mit ihm werden die Außengrenzen bzw. das innere Feld der Disziplin 
abgesteckt. Dieser, innere Ort' zeigt sich in dem, was Jakob Ossner unter Verweis auf Ludwik Fleck einen gemeinsamen „Denkstil“ nennt, der sich durch eine klare Trennung zwischen den Zielen (von Deutschunterricht), der Erstellung von Zielsystemen und der Begründung der Handlungsalternativen zur Erreichung der Ziele auszeichnet (Ossner 2001, S. 29).

\section{Ein Besuch im Ortskern}

Deutschdidaktik hat als Wissenschaft ein besonderes Verhältnis zur Praxis des Deutschunterrichts. In diesem Bezug wird auch der Kern dessen, was ein gemeinsamer „Denkstil“ sein könnte, deutlich. Mitunter wird in diesem Kontext auch von der Deutschdidaktik als einer praxisorientierten, praxisbezogen oder - darüber hinaus gar - „eingreifenden“ Wissenschaft gesprochen (z. B. Kepser 2013). Ossner hat 1993 und 2001 hingegen ein Konzept vorgelegt, in welchem er die Deutschdidaktik im Kern als „praktische Wissenschaft“ bestimmt:

Eine praktische Wissenschaft erfüllt sich nicht in der Episteme, der Erklärung des Seienden, ihre Gegenstände der Erkenntnis sind im Werden. [...] Es geht um die Bewältigung der Aufgabe, ein Können im Gegenstandsfeld Sprache auszubilden. (Ossner 1993, S. 190-192)

In dieser Konzeption ist bereits ein bestimmter Denkstil angelegt: Die bei Ossner genannten Aufgaben sind in Lehre und Forschung "für Lehrer" zu denken (Ossner 1993, S. 197). Das heißt: Deutschdidaktik kann nicht unabhängig von denen, die sie studieren, entworfen werden. Sie ist dementsprechend eine "gelehrte Wissenschaft“. Im Hinblick auf Fragen der Lehre geht es dann um die Vermittlung von

- Wissen im „Handlungsfeld“, also das „didaktische Brauchtum“ bzw. die „in Frage kommenden Handlungen“" (gedacht auch als Repertoire von Handlungsalternativen).

- Wissen über die SchülerInnen auf der Basis einer gegenstandsorientierten, psychologischen und soziologischen Forschung.

- Wissen über das Gegenstandsfeld, also Funktion und genetische Struktur des Gegenstandsfeldes (ebd.).

Mit „didaktischem Brauchtum“2 ist hier ein Kulturgut gemeint, mit dem Handelnde in variablen Situationen eine geeignete Handlung finden sowie sie gegen andere Handlungen abwägen. Es geht um solche Handlungen, die sich - bezogen auf die Struktur des zu vermittelnden Gegenstandes sowie in Hinblick auf die SchülerInnen - bewährt haben (ebd., S. 194). Vor diesem Hintergrund spricht

2 Ivo $(1975 ; 1977)$ spricht von „unterrichtlichem“ und „erzieherischem Brauchtum“. 
Ossner dann 2001 von einer „Deutschdidaktik als Handlungswissenschaft“ (ebd., S. 24). Frederking (2014) merkt dazu an:

Allerdings ist diese wissenschaftstheoretische Verortung ebenfalls nicht ganz unproblematisch. Denn die Deutschdidaktik erforscht nicht nur Handlungen, sondern auch deren Grundlagen. Der Deutschdidaktik geht es in der Forschung mithin nicht nur um Anwendungsorientierung, sondern immer auch um Grundlagenforschung. In diesem spezifischen Forschungsprofil ähnelt sie als Wissenschaft der Medizin. Ist das Heilen das praktische Ziel der Medizin, so ist erfolgreiches Lehren und Lernen das praktische Ziel der Deutschdidaktik (bzw. der Fachdidaktiken im Allgemeinen). (Frederking 2014, S. 113)

Zwei Aspekte sind hier anzumerken bzw. bereits bei Ossner (1993) zu finden: Die Analogie zur Medizin passt - erstens - nur begrenzt: „Ihr kann man nicht ohne weiteres unterstellen, daß sie die Krankheiten, die sie kuriert, auch selbst produziert" (ebd., S. 193).

Darüber hinaus heißt es bei Ossner (1993, S. 193) zudem - zweitens -, dass empirische Forschung in der Didaktik notwendigerweise auf das zielt, was in Unterrichtsprozessen oder für den Unterricht von Bedeutung ist (ebd.). So interessieren beispielsweise weniger irgendwelche (beliebigen) Fähigkeiten, die SchülerInnen in den Unterricht einbringen, als vielmehr solche Fähigkeiten, die sie unter den Bedingungen von Deutschunterricht (weiter)entwickeln. In dieser Perspektive ist bei einer praktischen Wissenschaft dann auch Grundlagenforschung mitgemeint (vgl. dazu auch Ossner 2001, S. 30). Eine notwendige Bedingung von Grundlagenforschung ist allerdings der Bezug auf Entscheidungssituationen (im Deutschunterricht) (Ossner 1993, S. 193). Diese werden zerlegt in Handlung und Folge einer Handlung sowie die möglichen Umstände (Bedingungen), die die Folge beeinflussen (ebd.). Hinzu kommen dann noch „die subjektiv angesetzten Wahrscheinlichkeiten für das Eintreffen der möglichen Umstände.“ (ebd.)

Dass bei Ossner auch Grundlagenforschung mitgemeint ist, lässt sich an einem Beispiel diskutieren: Vogt hat 2002 von der Lehrkraft geplante Formen des Diskutierens in der Primar- und Sekundarstufe untersucht. Die Arbeit zielt u. a. darauf ab, mit linguistischen Mitteln den Erwerb eines „Diskussionsbewusstseins “ nachzuzeichnen (ebd., S. 305 f.). Dabei kommt Vogt verkürzt zu folgenden Ergebnissen:

1. In der Primarstufe gewöhnten sich die SchülerInnen erst langsam an die Bedingungen, unter denen ein für Gruppen organisierter Lehr-Lernprozess stattfindet (ebd., S. 259). In Teilen werden Regeln der Gesprächsorganisation beherrscht und die Aufmerksamkeitssteuerung auf den Redner ausgerichtet. Die sprachlichen Handlungen seien noch sehr durch spontansprachliche Äußerungen geprägt. 
2. In der unteren Sekundarstufe I (fünfte bis sechste Jahrgangsstufe) wachse das Interesse an thematischen Zusammenhängen - zunehmend stellten sich die Schülerinnen und Schüler zu diesem Zeitpunkt auf ihren Partner ein und lernten, die situativen Bedingungen der Unterrichtssituation zu reflektieren. Es entwickle sich ein reflektierter Zugriff auf Gegenstände und Sachverhalte, weg von einem anschaulich-erlebnishaften Zugriff. Die Beteiligten bildeten so etwas wie eine „sprachlich-kommunikative Identität“ aus (ebd., S. 291).

3. Mit Eintritt in die obere Sekundarstufe I (achte bis zehnte Jahrgangsstufe) reduzierten sie dann ihre Spontaneität, SchülerInnen neigten nun eher dazu, ihre Subjektivität vor der Klassenöffentlichkeit zu verbergen. Gleichzeitig steige das Interesse an thematischer und personaler Differenzierung (i. S. v. „Identitätskrisen“; ebd., S. 305). Zwei „Diskussionswelten“ seien jetzt im Unterricht leitend: ein gelenktes Unterrichtsgespräch und kontroverse Formen, insbesondere Pro-Kontra-Diskussionen (ebd., S. 305).

4. Schließlich könnten SchülerInnen in der Adoleszenz (Sekundarstufe II) distanziert mit sprachlichen Mitteln umgehen, sie seien selbst-reflexiv, indem sie Formen sprachlicher Mehrdeutigkeit wie Ironie oder Satire erprobten und über die Fähigkeit verfügten, auch fremde Perspektiven einzunehmen. Im Rahmen variierender Gesprächstypen bilde sich eine „Diskussionsbewusstheit“ (ebd., S. 306).

Vogts Analysen von 2002 zielen - im Sinne Ossners (1993) - nicht auf irgendwelche Entwicklungsprozesse. Die Fähigkeiten sind auch nicht „das Ergebnis einer kognitiven Reifung, die unabhängig von Unterweisungen stattgefunden hätte“ (Ossner 1993, S. 196). Vielmehr zielen die Analysen darauf ab, grundlegendes Wissen bereitzustellen über einen bestimmten Typ Handlung (von SchülerInnen) und deren Folgen zu unterschiedlichen Zeitpunkten und unter verschiedenen Bedingungen im schulischen Bildungsverlauf. Vogts Arbeit ist also in mancherlei Hinsicht deutschdidaktische Grundlagenforschung im Sinne einer praktischen Handlungswissenschaft, auch wenn Vogt die unterrichtlichen Rahmenbedingungen, unter denen sich Lernentwicklungen nachzeichnen lassen, zu wenig beschreibt und reflektiert.

\section{Ausblick: Kann ein Angebot-Nutzungs-Modell Impulse liefern?}

Um das innere Feld einer solchen praktischen Handlungswissenschaft genauer auszuleuchten, braucht man Bezugsgrößen. Diese ergeben sich aus den wesentlichen Faktoren, Prozessen und Akteuren des Deutschunterrichts. Es geht also 
im Kern um die Lehrkräfte und SchülerInnen im Handlungsfeld, die relevanten Gegenstände bzw. das Gegenstandsfeld sowie die jeweiligen Kontexte (Rahmenbedingungen der Schule, Klasse etc.) und die individuellen und familiären Voraussetzungen.

Damit bestehen Ähnlichkeiten mit Modellen, die auch in anderen Disziplinen bzw. in der Pädagogik diskutiert werden. Ein prominentes Modell stammt hier von Helmke (2012, S. 69 f.): In dem so genannten „Angebot-Nutzungs-Modell“ wird Unterricht als Wirkungsgeflecht beschrieben. Es umfasst Merkmale der Lehrperson, der SchülerInnen und des Unterrichts. Der Unterricht repräsentiert in diesem Modell lediglich ein Angebot, das nicht notwendigerweise auch zu den von der Lehrkraft intendierten Wirkungen führen muss. Gegebenenfalls zeigt er gar keine Wirkungen. Lernwirksamkeiten hängen demnach von verschiedenen Prozessen und Faktoren, vom Kontext und den jeweiligen AkteurInnen (also auch von den SchülerInnen selbst) ab. Es spielt etwa eine entscheidende Rolle, ob und wie die Akteure (Lehrkräfte und SchülerInnen) die gegenseitigen Unterrichtsangebote nutzen (vgl. Abbildung 2).

Abb. 2: Angebot-Nutzungs-Modell (u.a. Helmke 2012)

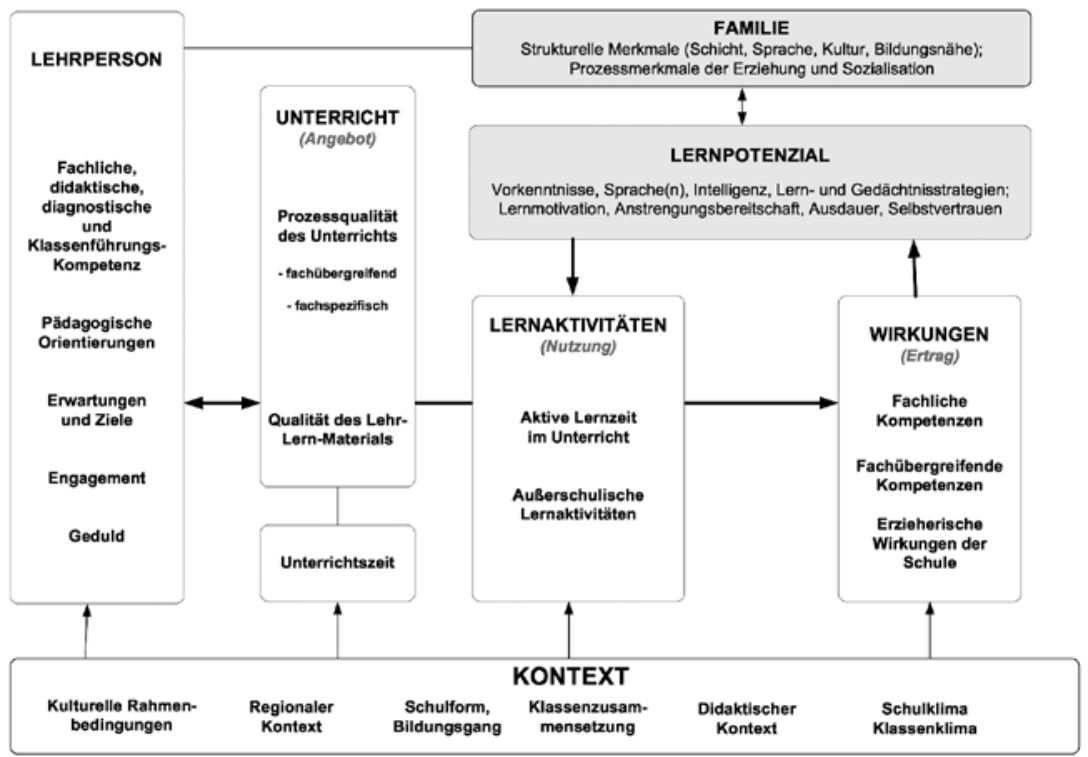


Hier geht es um ein fachunspezifisches Modell. Es lässt sich aber auf deutschdidaktische Fragen beziehen, insbesondere wenn man die Angebotsseite spezifiziert. Hier ließen sich die jeweiligen Domänen (integrativ oder fachübergreifend) genauso modellieren wie auch die Art der eingesetzten Materialien, Aufgaben und Methoden sowie das didaktische Brauchtum. Auf Seite der Wirkungen wären dann u. a. die fachlichen Kompetenzen aufzuführen, die SchülerInnen sprachlich, literarisch und medienspezifisch aufweisen bzw. die sie dann in konkreten Situationen nutzen. Dann könnte es gleichsam für die Forschung wie die Lehrerbildung impulsgebend sein. Auf beiden Seiten ginge es dann viel mehr als bisher um das Geflecht von Angebot, Nutzung und Ertrag. Als ein solches Geflecht sind dann auch die Gegenstände des Deutschunterrichts zu denken. Das Angebot-NutzungsModell von Helmke soll hier natürlich nicht einfach in ein deutschdidaktisches Modell umgemünzt werden. Helmkes Modell kann aber ein Impulsgeber für einen gemeinsamen Denkstil sein, um das innere Feld einer praktischen Handlungswissenschaft genauer auszuleuchten.

Dieses „Ausleuchten“ steht in mancherlei Hinsicht noch aus. Eine solche deutschdidaktische Arbeit würde sich aber mit Blick auf eine größere Verständigung der KollegInnen ,im Ortskern' lohnen. Die Deutschdidaktik wäre dann perspektivisch viel weniger eine $z u$ gestaltende als eine gestaltende Wissenschaft.

\section{Literatur}

Abraham, Ulf/Kepser, Matthis (2005): Literaturdidaktik Deutsch. Eine Einführung. Berlin: Erich Schmidt Verlag.

Bräuer, Christoph (2011): Die Unterrichtsrahmenanalyse - ein Beobachtungsinstrument für die praktische Forschung. In: Osnabrücker Beiträge zur Sprachtheorie. 15. Jg., H. 80, S. 13-30.

Bremerich-Vos, Albert (1993): Zur Einführung. In: ders. (Hrsg.): Handlungsfeld Deutschunterricht im Kontext. Frankfurt a. M.: Diesterweg, S. 1-7.

Frederking, Volker (2014): Deutschdidaktik als transdisziplinäre, anwendungsund grundlagenorientierte empirische Wissenschaft. In: Mitteilungen des Deutschen Germanistenverbandes. Jg. 61. H. 2, S. 109-119.

Hebel, Franz (1993): Handlungsfelder der germanistischen Didaktikwissenschaft. In: Bremerich-Vos, Albert (Hrsg.): Handlungsfeld Deutschunterricht im Kontext. Festschrift für Hubert Ivo. Frankfurt a. M.: Diesterweg, S. 200-214.

Helmke, Andreas (2012): Unterrichtsqualität und Lehrerprofessionalität: Diagnose, Evaluation und Verbesserung des Unterrichts. Seelze-Velber: Kallmeyer. 
Ivo, Hubert (1975): Handlungsfeld Deutschunterricht. Argumente und Fragen einer praxisorientierten Wissenschaft. Frankfurt a. M.: Fischer.

Ivo, Hubert (1977): Zur Wissenschaftlichkeit der Didaktik der deutschen Sprache und Literatur. Frankfurt a. M.: Diesterweg. 1977.

Kepser, Matthis (2013): Deutschdidaktik als eingreifende Kulturwissenschaft. Ein Positionierungsversuch im wissenschaftlichen Feld. In: Didaktik Deutsch. Jg. 2013. H, 34, S. 52-68.

Mittelstraß, Jürgen (1992): Leonardo-Welt. Über Wissenschaft, Forschung und Verantwortung. Frankfurt am. M: Suhrkamp.

Müller-Michaels, Harro (2001): Deutschunterricht im Spannungsfeld von Wissenschaft, Kultur und Leben. In: Rosebrock, Cornelia/Fix, Martin (Hrsg.): Tumulte. Deutschdidaktik zwischen den Stühlen. Baltmannsweiler: Schneider, S. 33-41.

Müller-Michaels, Harro (2009): Grundkurs Lehramt Deutsch: Germanistik, Sicher im Studium. Stuttgart: Klett.

Ossner, Jakob (2001): Elemente eines Denkstils für didaktische Entscheidungen. In: Rosebrock, Cornelia/Fix, Martin (Hrsg.): Tumulte. Deutschdidaktik zwischen den Stühlen. Baltmannsweiler: Schneider, S. 17-32.

Ossner, Jakob (1993): Praktische Wissenschaft. In: Bremerich-Vos, Albert (Hrsg.): Handlungsfeld Deutschunterricht im Kontext. Frankfurt a. M.: Diesterweg, S. 186-199.

Pöhlmann, Claudia/Neumann, Daniela/Tesch, Bernd/Köller, Olaf (2009): Die Bildungsstandards im allgemeinbildenden Schulsystem. In: Köller, Olaf/Knigge, Michel/Tesch, Bernd (Hrsg.): Sprachliche Kompetenzen im Ländervergleich. Überprüfung der Erreichung der Bildungsstandards für den Mittleren Schulabschluss für Deutsch und die erste Fremdsprache in der neunten Jahrgangsstufe. Münster: Waxmann, S. 13-19.

Rosebrock, Cornelia (2001): Einleitung. In: Rosebrock, Cornelia/Fix, Martin (Hrsg.): Tumulte. Deutschdidaktik zwischen den Stühlen. Baltmannsweiler: Schneider, S. 2-7.

Rosebrock, Cornelia/Fix, Martin (Hrsg.) (2001): Tumulte. Deutschdidaktik zwischen den Stühlen. Baltmannsweiler: Schneider.

Schneider, Wolfgang/Baumert, Jürgen/Becker-Mrotzek, Michael/Hasselhorn, Marcus/Kammermeyer, Gisela/Rauschenbach Thomas/Roßbach, Hans-Günther/ Roth, Hans-Joachim/Rothweiler, Monika/Stanat, Petra (2012): Expertise „Bildung durch Sprache und Schrift (BISS)“ (Bund-Länder-Initiative zur Sprachförderung, Sprachdiagnostik und Leseförderung). http://www.biss-sprachbildung. de/pdf/BiSS-Expertise.pdf. Abgerufen am 01.08.2016. 
SDD (2014): Ausschreibungstext zum Tagungsthema. Normen - Erwartungsmuster zwischen Orientierung und Begrenzung. Symposion Deutschdidaktik 7.-11. 9. 2014. http://www.fhnw.ch/ph/zl/sdd2014/tagungsthema. Abgerufen am 9.01.2017.

Vogt, Rüdiger (2002): Im Deutschunterricht diskutieren. Zur Linguistik und Didaktik einer kommunikativen Praktik. Tübingen: Niemeyer.

Winkler, Iris (2012): Wozu Literaturdidaktik? Perspektiven auf eine Disziplin zwischen den Stühlen. In: Doering, Sabine / Wätjen, Hans-Joachim (Hrsg.): Oldenburger Universitätsreden. Nr. 200. BIS-Verlag: Oldenburg, S. 9-31. URL: http://oops.uni-oldenburg.de/1502/1/ur200.pdf. Abgerufen am 01.04.2016. 